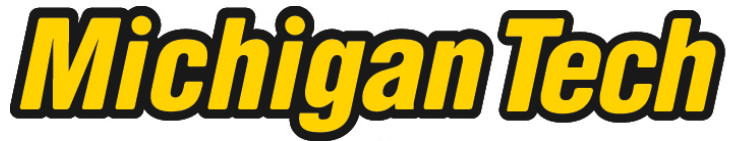 \\ Michigan Technological University Create the Future Digital Commons @ Michigan Tech
}

Dissertations, Master's Theses and Master's Reports - Open

Dissertations, Master's Theses and Master's

Reports

2013

\section{A SCR Model based on Reactor and Engine Experimental Studies for a Cu-zeolite Catalyst}

Xiaobo Song

Michigan Technological University

Follow this and additional works at: https://digitalcommons.mtu.edu/etds

Part of the Mechanical Engineering Commons

Copyright 2013 Xiaobo Song

\section{Recommended Citation}

Song, Xiaobo, "A SCR Model based on Reactor and Engine Experimental Studies for a Cu-zeolite Catalyst", Dissertation, Michigan Technological University, 2013.

https://doi.org/10.37099/mtu.dc.etds/582

Follow this and additional works at: https://digitalcommons.mtu.edu/etds

Part of the Mechanical Engineering Commons 


\title{
A SCR MODEL BASED ON REACTOR AND ENGINE EXPERIMENTAL STUDIES FOR A CU-ZEOLITE CATALYST
}

\author{
By \\ Xiaobo Song \\ A DISSERTATION \\ Submitted in partial fulfillment of the requirements for the degree of \\ DOCTOR OF PHILOSOPHY
}

In Mechanical Engineering - Engineering Mechanics

MICHIGAN TECHNOLOGICAL UNIVERSITY

2013 

This dissertation has been approved in partial fulfillment of the requirements for the Degree of DOCTOR OF PHILOSOPHY in Mechanical Engineering - Engineering Mechanics

Department of Mechanical Engineering - Engineering Mechanics

Dissertation Co-Advisor: $\quad$ Dr. John H. Johnson

Dissertation Co-Advisor: $\quad$ Dr. Jeffrey D. Naber

Committee Member: Dr. Gordon P. Parker

Committee Member: Dr. Jason M. Keith

Department Chair: $\quad$ Dr. William Predebon 



\section{Table of Contents}

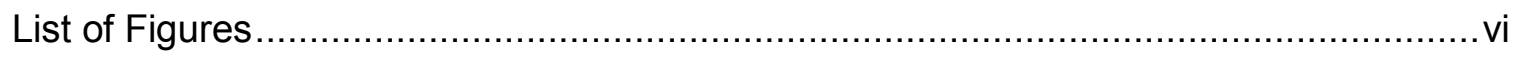

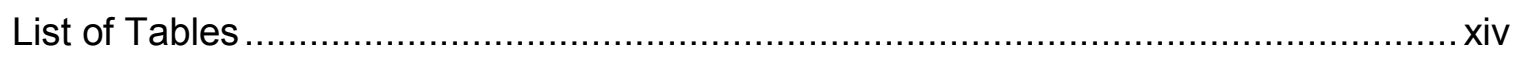

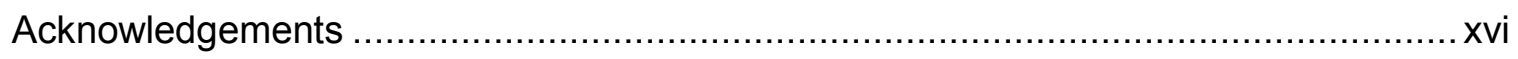

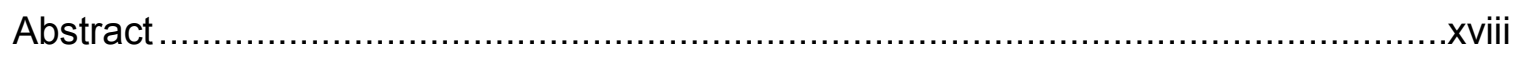

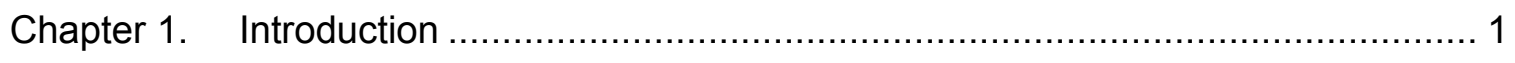

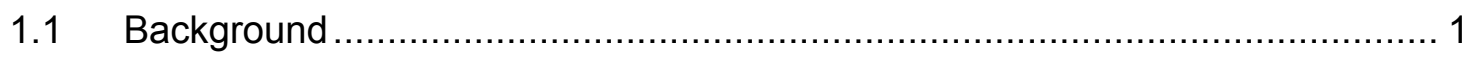

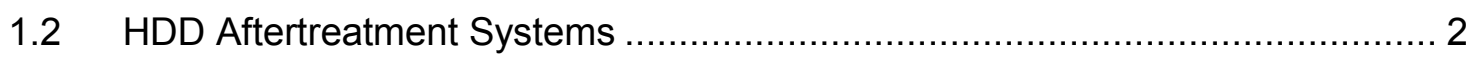

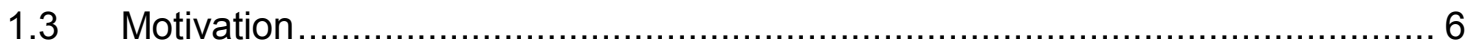

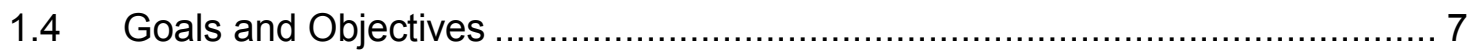

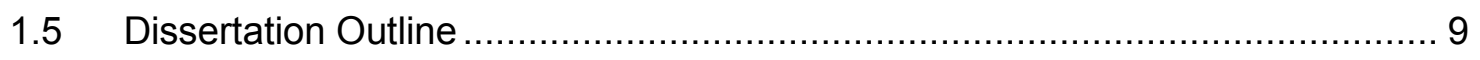

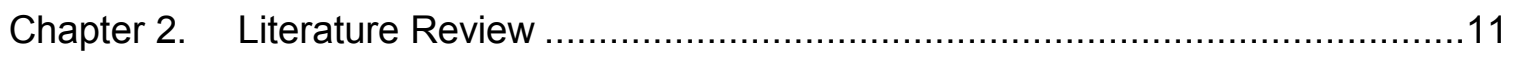

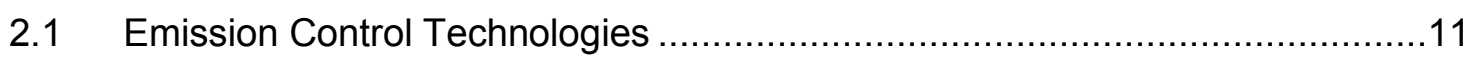

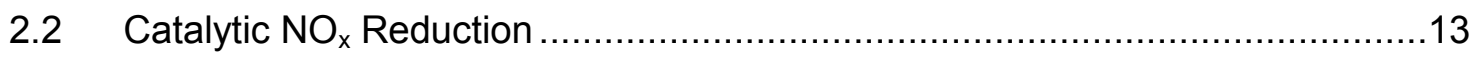

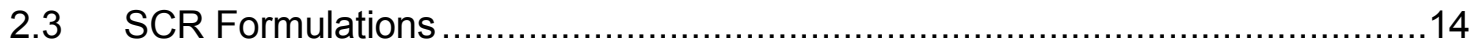

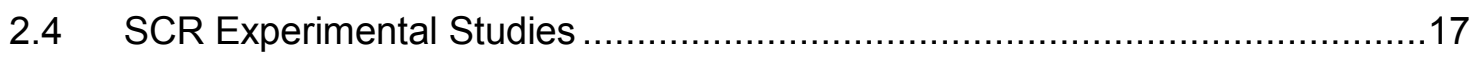

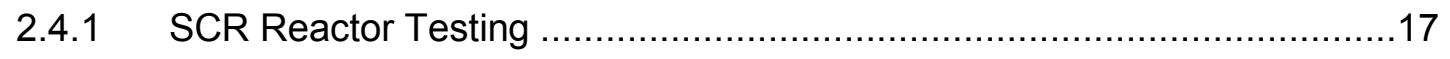

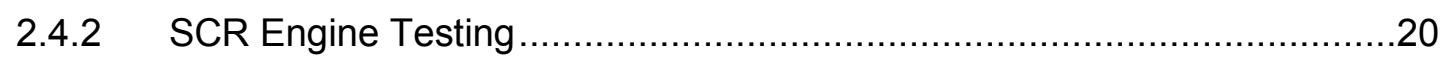

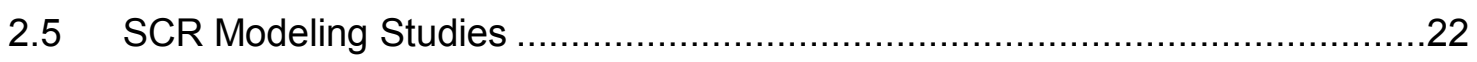

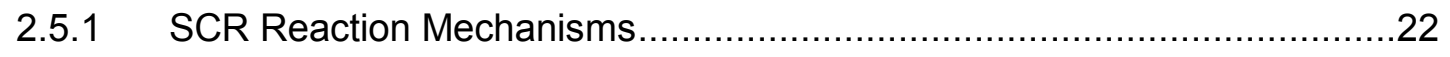

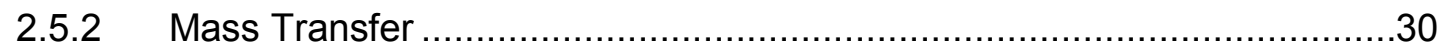

2.5.3 Heat and Energy Transfer .............................................................. 33

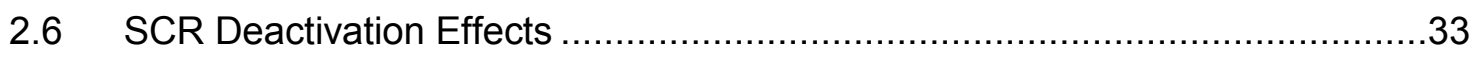

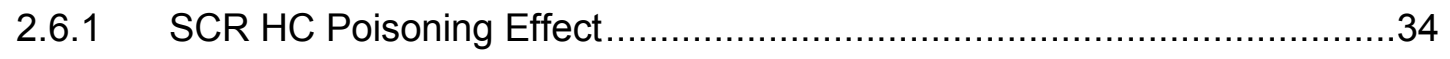




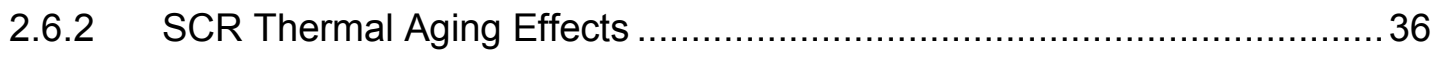

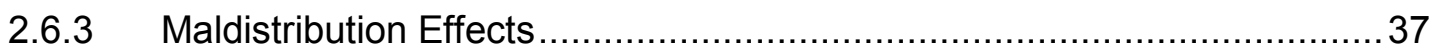

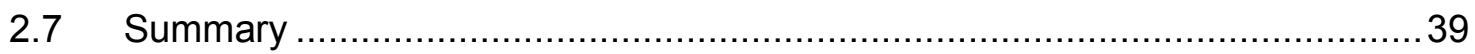

Chapter 3. Experimental Setup and Test Procedures......................................... 42

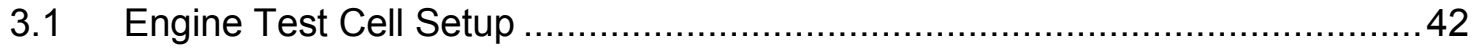

3.2 Engine, Fuel and Aftertreatment System.................................................. 43

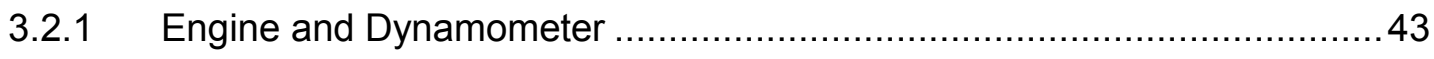

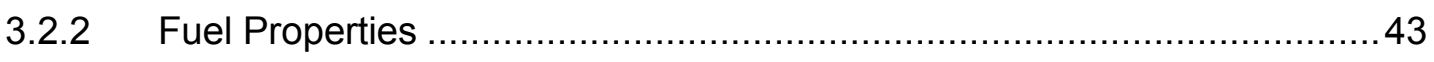

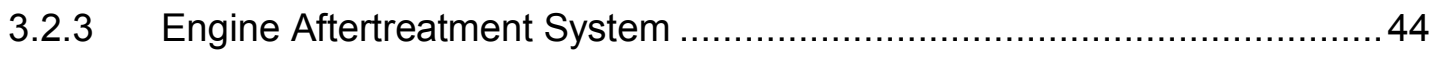

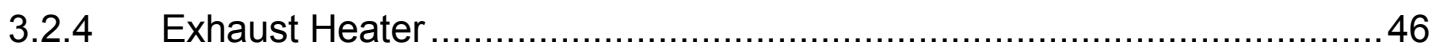

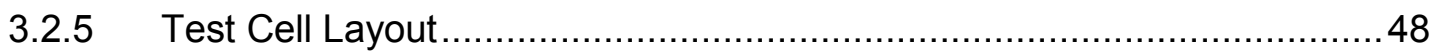

3.3 Measurements and Data Acquisition System ……..................................... 49

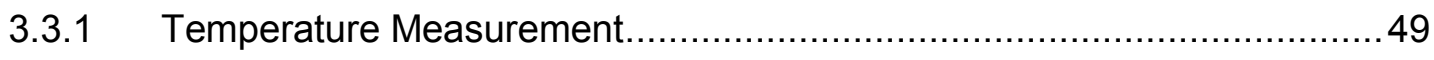

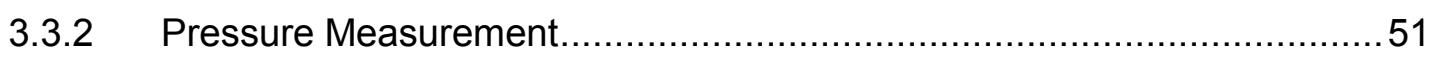

3.3.3 Exhaust Mass Flow Rate Measurement.............................................. 51

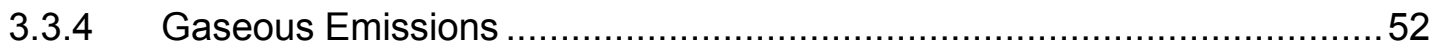

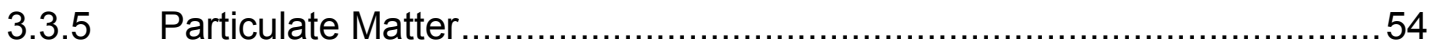

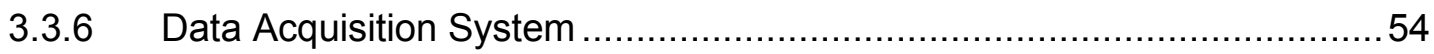

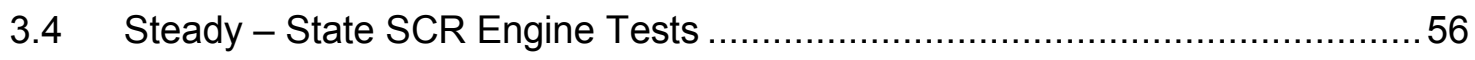

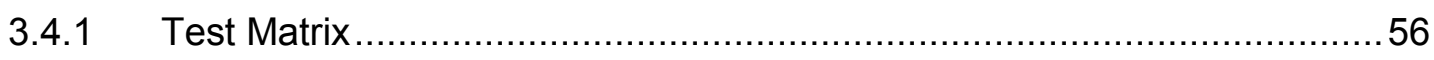

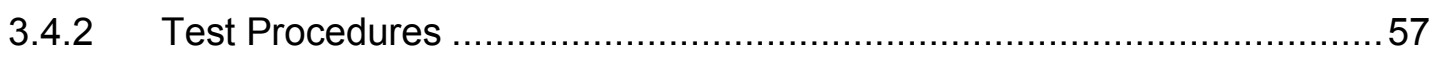

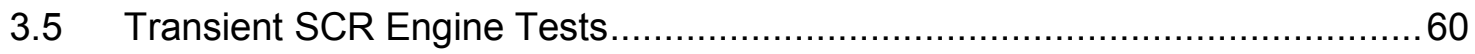

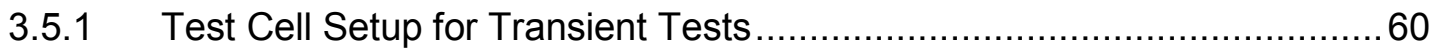

3.5.2 Surrogate HD-FTP Cycle Development …………............................... 61

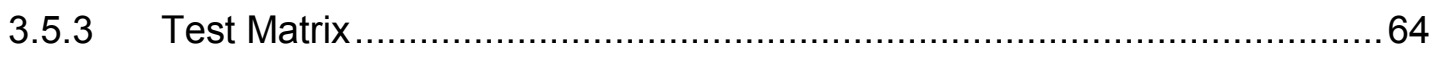

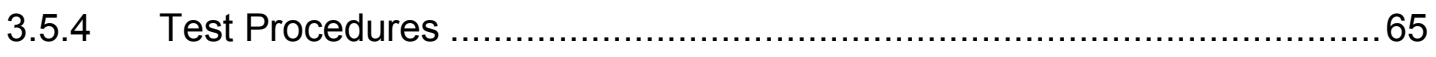




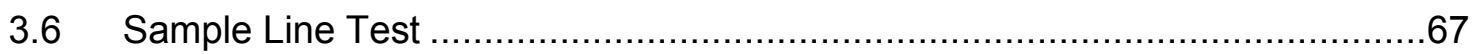

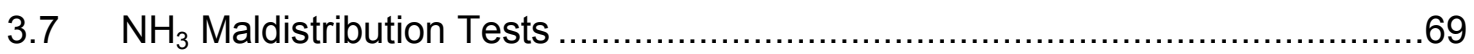

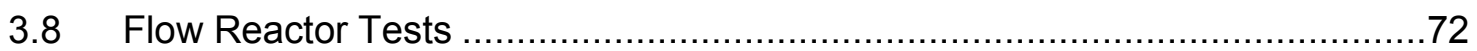

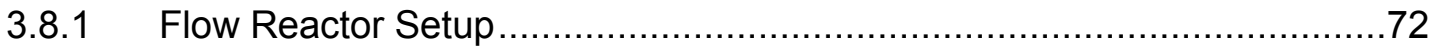

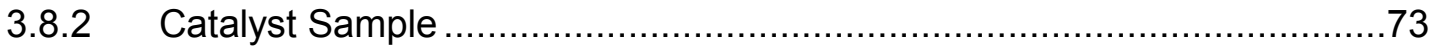

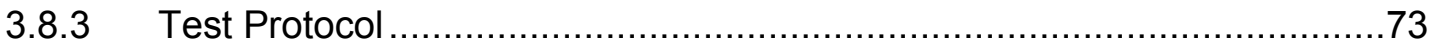

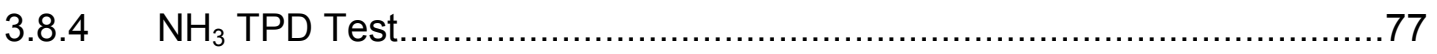

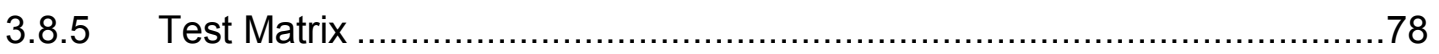

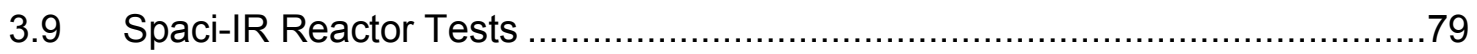

Chapter 4. SCR Model Development and Calibration ......................................... 82

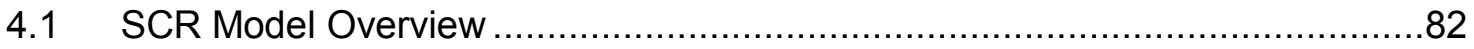

4.1.1 Mass Transfer Equations........................................................ 83

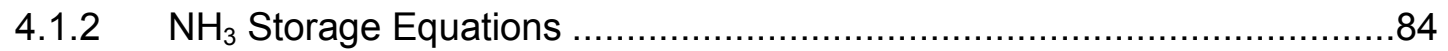

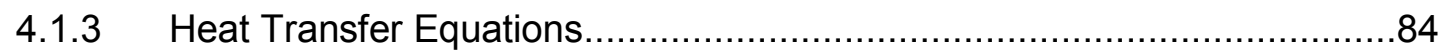

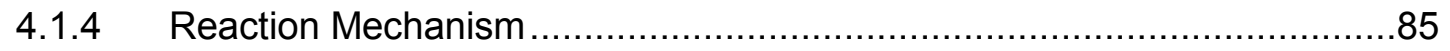

4.2 SCR Model Calibration with Spaci-IR Data .......................................... 88

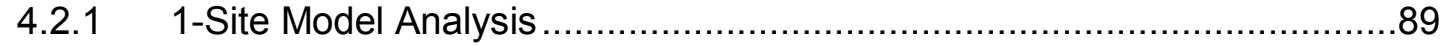

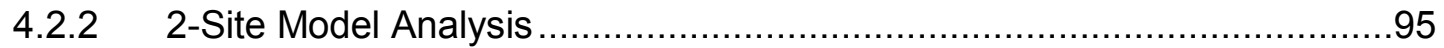

4.3 SCR Model Calibration with Flow Reactor Data ..................................99

4.3.1 Model Calibration Procedures ......................................................... 99

4.3.2 $\quad \mathrm{NH}_{3}$ Storage Parameters ................................................... 101

4.3.3 $\quad \mathrm{NH}_{3}$ Oxidation Parameters ......................................................... 104

4.3.4 $\mathrm{NO}+\mathrm{NH}_{3}$ SCR Reaction Parameters..........................................

4.3.5 $\quad \mathrm{NO}_{2}+\mathrm{NH}_{3}$ SCR Reaction Parameters ........................................ 106

4.3.6 $\quad \mathrm{NO}_{\mathrm{x}}+\mathrm{NH}_{3}$ SCR Reaction Parameters ........................................ 107

$4.4 \quad$ Sample Line Model Development ..................................................109 
4.5 SCR Model Calibration with Engine Data .......................................... 112

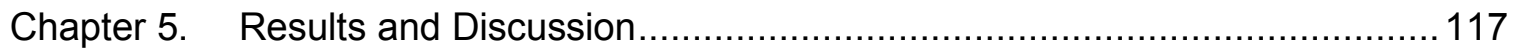

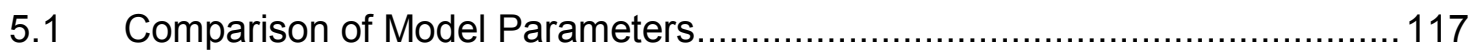

5.2 SCR Model Calibrated to Reactor Data............................................... 122

5.2.1 Model Simulation of TPD Experiments .......................................... 123

5.2.2 Model Simulation of Isothermal Reactor Experiments ......................... 125

5.3 SCR Model Calibrated to Engine Data ........................................... 128

5.3.1 Model Simulation of Steady State Engine Experiments...................... 128

5.3.2 Model Simulation of Transient Engine Experiments ............................ 131

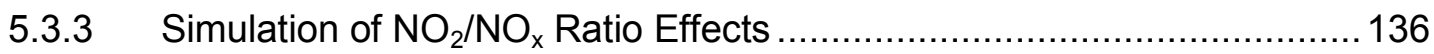

$5.4 \mathrm{NH}_{3}$ Maldistribution Data and Simulation of Maldistribution Effects .............. 138

5.4.1 $\quad \mathrm{NH}_{3}$ Maldistribution Experimental Results ................................... 138

5.4.2 $\mathrm{NH}_{3}$ Maldistribution Effects on SCR Performance ............................. 145

5.4.3 Maldistribution Effects on SCR Model Parameters............................. 149

5.5 SCR Thermal Aging Data and Effects ............................................ 152

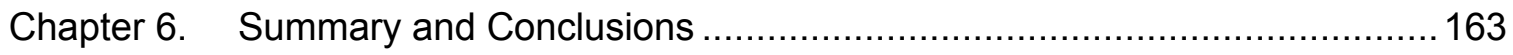

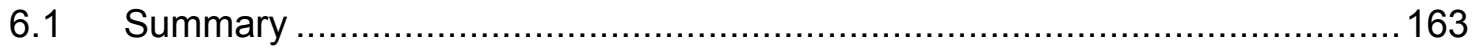

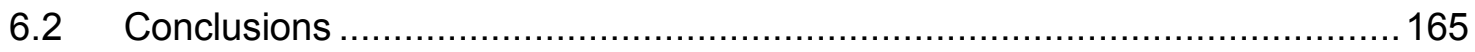

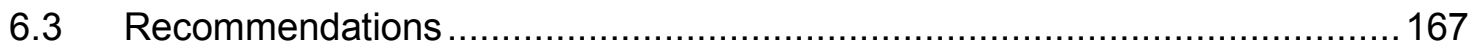

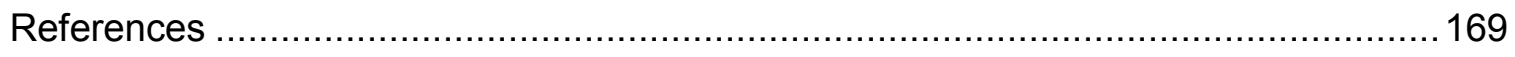

Appendix A. IMR-MS Start up and Calibration procedures ............................... 181

Appendix B. Calibration of the DEF Injector........................................... 182

Appendix C. Calculation of SCR Actual Space Velocity ................................. 184

Appendix D. Fuel Mass Flow Rate Calculation for Transient Test....................... 185

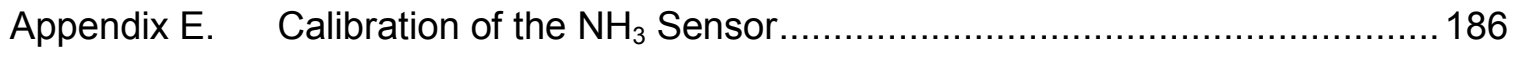

Appendix F. Processing of the Spaci-IR Data.......................................... 188 
Appendix G. Selection of Reactor Data for Model Calibration.............................193

Appendix $\mathrm{H}$. Simulation Results of TPD Experiments ....................................196

Appendix I. Simulation Results of Isothermal Reactor Tests ................................198

Appendix J. Simulations of Steady State Engine Experiments ...........................208

Appendix K. Simulation Results of Transient SCR Experiments .........................212

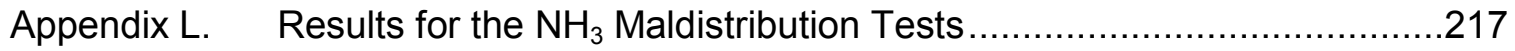

Appendix M. Permission to Use Copyrighted Material ...................................220 


\section{List of Figures}

Figure 1.1: An evolution of U.S. EPA and Europe regulations for HDD engines [14] ...... 2

Figure 1.2: Typical layout of HDD engine aftertreatment system ............................... 3

Figure 2.1: $\mathrm{NO}_{x}$ conversion performance at $200^{\circ} \mathrm{C}$ of $\mathrm{Fe}$ and $\mathrm{Cu}$-zeolite SCR with 350 ppm of $\mathrm{NO}$ and $350 \mathrm{ppm}$ of $\mathrm{NH}_{3}$ in the gas flow [50]. 16

Figure 2.2: A schematic of the Spaci-FTIR test setup: "(1, 2, 3): thermocouples; (4) hollow quartz rods for gas preheating and distribution; (5) quartz tube reactor; (6) monolith Fe-zeolite catalyst; (7) insulation material; (8) fused silica capillary."[63]

Figure 2.3: $\mathrm{NH}_{3}$ oxidation in the absence of $\mathrm{NO}_{x}$ as a function of temperature over a $\mathrm{Cu}$ based SCR under the space velocity of $30,000 \mathrm{hr}^{-1}$ [46]

Figure 2.4: Damage equations for reactor aging of Cu-chabazite SCR with space velocity of $30,000 \mathrm{hr}^{-1}$ and $5 \% \mathrm{H}_{2} \mathrm{O}$ [115]

Figure 2.5: $\mathrm{NO}_{x}$ conversion efficiency and $\mathrm{NH}_{3}$ slip as a function ANR uniformity [116]. 38

Figure 3.1: Picture of the diesel engine test cell at MTU.

Figure 3.2: The heating capability of the heater as a function of the exhaust mass flow rate going through the heater.

Figure 3.3: The $\mathrm{DOC}$ inlet $\mathrm{NO}_{2} / \mathrm{NO}_{\mathrm{x}}$ ratio as a function of the heating element

temperature

Figure 3.4: The DOC inlet PM concentration as a function of the heating element temperature. .48

Figure 3.5: Schematic of the ISB engine test cell setup with instrumentation 49

Figure 3.6 DOC thermocouple layout (Unit: inch) 50

Figure 3.7: CPF thermocouple layout (Unit: inch). .50

Figure 3.8: SCR thermocouple layout (Unit: inch) .51

Figure 3.9: Production $\mathrm{NO}_{\mathrm{x}}$ sensor.... .54

Figure 3.10: Delphi ammonia sensor... .54

Figure 3.11: Flow chart of SCR test procedures. .58

Figure 3.12: Scheduled SCR inlet ANR for steady state SCR engine test 60 
Figure 3.13: Switches used for switching between manual control mode and external voltage control mode.

Figure 3.14: Engine conditions of the HD-FTP cycle and the developed surrogate HDFTP cycle.

Figure 3.15: Comparison the experimental measurements for different runs of the developed surrogate HD-FTP cycle

Figure 3.16: SCR inlet $\mathrm{NO}, \mathrm{NO}_{2}$ concentrations, SCR inlet temperature, and SCR standard space velocity during the surrogate HD-FTP cycle .....................64

Figure 3.17: Engine conditions and control voltages for the transient test...................66

Figure 3.18: Comparison of $\mathrm{NH}_{3}$ slip measurements from the IMR-MS, the $\mathrm{NH}_{3}\left(\mathrm{NH}_{3}\right.$,

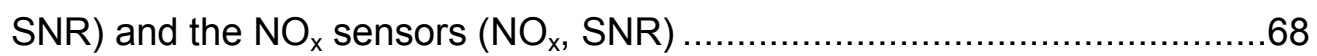

Figure 3.19: Sampling probes for different radial measurement locations ......................70

Figure 3.20: Gaseous sampling positions at the outlet face of the 1st SCR brick..........71

Figure 3.21: Test protocol showing the inlet gas concentrations for reactor testing........74

Figure 3.22: Test protocol for $\mathrm{NH}_{3}$ TPD experiments ......................................77

Figure 3.23: Test protocol of the Spaci-IR reactor tests .......................................79

Figure 4.1: Overconsumption of $\mathrm{NH}_{3}$ with respect to $\mathrm{NO}$ under standard SCR conditions and $\mathrm{NH}_{3}$ oxidized during step $3\left(\right.$ Space Velocity $\left.=60,000 \mathrm{hr}^{-1}\right) \ldots \ldots \ldots \ldots \ldots . . . .88$

Figure 4.2: $\mathrm{NH}_{3}$ storage at each test temperature ..........................................

Figure 4.3: 1-site model simulated $\mathrm{NO}_{\mathrm{x}}$ concentration at different axial locations at equilibrium state of $\mathrm{NO}_{x}+\mathrm{NH}_{3}$ reaction section compared to experimental results

Figure 4.4: 1-site model simulated $\mathrm{NH}_{3}$ concentration at different axial locations at equilibrium state of $\mathrm{NO}_{x}+\mathrm{NH}_{3}$ reaction section compared to experimental results

Figure 4.5: Simulated cumulative $\mathrm{NH}_{3}$ stored for the 1-site model as a function of axial position compared to experimental results

Figure 4.6: 2-site model simulated $\mathrm{NO}_{\mathrm{x}}$ concentration at different axial locations at equilibrium state of $\mathrm{NO}_{x}+\mathrm{NH}_{3}$ protocol section compared to experimental results .97 
Figure 4.7: 1-site model simulated $\mathrm{NH}_{3}$ concentration at different axial locations at equilibrium state of $\mathrm{NO}_{x}+\mathrm{NH}_{3}$ protocol section compared to experimental results.

Figure 4.8: Simulated cumulative $\mathrm{NH}_{3}$ stored of the 1-site model as a function of axial position compared to experimental results. .99

Figure 4.9: $\mathrm{NH}_{3}$ stored on the catalyst at step 2 of the protocol for each reactor test condition 102

Figure 4.10: Arrhenius plot of reaction rate constants for $\mathrm{NH}_{3}$ adsorption and desorption on two sites (Square: SV $=60,000 \mathrm{hr}^{-1}$, Diamonds: $90,000 \mathrm{hr}^{-1}$, Circle: $\left.120,000 \mathrm{hr}^{-1}\right)$..... 103

Figure 4.11: Arrhenius plot of reaction rate constants for $\mathrm{NH}_{3}$ oxidation reactions (Square: SV = 60,000 hr-1 , Diamonds:90,000 hr-1, Circle: 120,000 hr ${ }^{-1}$ ) ... 104

Figure 4.12: Arrhenius plot of reaction rate constants for the two standard SCR reactions and NO oxidation reaction (Square: SV $=60,000 \mathrm{hr}^{-1}$, Diamonds:90,000 $\mathrm{hr}^{-1}$, Circle: $120,000 \mathrm{hr}^{-1}$ )..... 106

Figure 4.13: Arrhenius plot of reaction rate constants for slow SCR reactions and $\mathrm{N}_{2} \mathrm{O}$ formation reaction (Square: SV $=60,000 \mathrm{hr}^{-1}$, Diamonds: $90,000 \mathrm{hr}^{-1}$, Circle: $\left.120,000 \mathrm{hr}^{-1}\right)$. 107

Figure 4.14: Arrhenius plot of reaction rate constants for fast SCR reaction (Square: SV $=60,000 \mathrm{hr}^{-1}$, Diamonds: 90,000 $\mathrm{hr}^{-1}$, Circle: $120,000 \mathrm{hr}^{-1}$ ) 108

Figure 4.15: Sample line model predicted $\mathrm{NH}_{3}$ compared to the IMR-MS measure during $103.8 \mathrm{ppm} \mathrm{NH}_{3}$ calibration gas as a step input 111

Figure 4.16: Sample line model predicted $\mathrm{NH}_{3}$ compared to the IMR- MS measured $\mathrm{NH}_{3}$ using $\mathrm{NH}_{3}$ sensor measurement during engine test as the input (Red: $\mathrm{NH}_{3}$ sensor measurement, Green: IMR-MS measurement, Blue: Sample line model predictions)

Figure 4.17: Arrhenius plots of reaction rate constant for each reaction in the SCR model based on the engine data

Figure 5.1: Comparison of the kinetic parameters for adsorption and desorption reactions on site 1

Figure 5.2: Comparison of the kinetic parameters for adsorption and desorption reactions on site 2 
Figure 5.3: Comparison of the kinetic parameters for the standard SCR reactions ......121

Figure 5.4: Comparison of the kinetic parameters for the fast and slow SCR reactions

Figure 5.5: Comparison of SCR outlet $\mathrm{NH}_{3}$ between model simulation and TPD reactor experimental results for the space velocity of $60,000 \mathrm{hr}^{-1}$ 123

Figure 5.6: Model simulated $\mathrm{NH}_{3}$ coverage fraction for TPD reactor test for the space velocity of $60,000 \mathrm{hr}^{-1}$. 124

Figure 5.7: $\mathrm{SCR}$ outlet concentrations of $\mathrm{NO}, \mathrm{NO}_{2}, \mathrm{NH}_{3}$, and $\mathrm{N}_{2} \mathrm{O}$, comparison between SCR model simulation and experimental measurements. Test conditions: SV $=90,000 \mathrm{hr}^{-1}$, Temperature $=300^{\circ} \mathrm{C}$. 126

Figure 5.8: Calculated $\mathrm{NH}_{3}$ stored on the catalyst during certain steps of the reactor protocol for the SV of $90,000 \mathrm{hr}^{-1}$ and the temperature of $300^{\circ} \mathrm{C}$ 128

Figure 5.9: Comparison of the SCR outlet gaseous concentrations between simulation results and experimental measurements for test point 2 in Table 3.11 with the temperature of $376^{\circ} \mathrm{C}$. (Sim1: SCR model predicted $\mathrm{NH}_{3} ; \operatorname{Sim} 2: \mathrm{NH}_{3}$ slip processed by the sample line model).

Figure 5.10: Model simulation compared to experimental results for the surrogate HDFTP transient test 1 with manual control of DEF injection 132

Figure 5.11: Cumulative $\mathrm{NO}_{x}, \mathrm{NH}_{3}$ and $\mathrm{NO}_{\mathrm{x}}$ conversion efficiency of the surrogate $\mathrm{HD}-$ FTP cycle, compare between simulation and experimental results for transient test 1 133

Figure 5.12: Model predicted $\mathrm{NH}_{3}$ storage on the catalyst during surrogate HD-FTP cycle tests 1-6. 135

Figure 5.13: Model predicted SCR outlet temperature for the surrogate HD-FTP test..136

Figure 5.14: Distribution profile of NO with the average ANR of 0 for test point $1 \ldots \ldots . .139$

Figure 5.15: Distribution profile of $\mathrm{NO}_{2}$ with the average ANR of 0 for test point $1 \ldots \ldots .140$

Figure 5.16: Distribution profile of $\mathrm{NO}_{\mathrm{x}}$ with the average ANR of 0 for test point $1 \ldots \ldots .140$

Figure 5.17: Distribution profile of NO with the average ANR of 0.3 for test point $1 \ldots . .141$

Figure 5.18: Distribution profile of $\mathrm{NO}_{2}$ with the average ANR of 0.3 for test point $1 \ldots .141$

Figure 5.19: Distribution profile of $\mathrm{NO}_{\mathrm{x}}$ with the average ANR of 0.3 for test point $1 \ldots .142$

Figure 5.20: Distribution profile of $\mathrm{NH}_{3}$ with the average ANR of 0.3 for test point $1 \ldots .142$ Figure 5.21: Distribution profile of ANR with the average ANR of 0.3 for test point $1 \ldots 143$ 
Figure 5.22: Distribution of ANR after installing swirl mixer for condition for test point 5

Figure 5.23: Schematic showing the setup of the model for maldistribution effects

simulation

Figure 5.24: Probability distribution function of maldistribution factors for the UI of 0.7 ,

0.8 and 0.9 146

Figure 5.25: Setup of the SCR inlet concentrations for simulation

Figure 5.26: $\mathrm{NO}_{x}$ reduction efficiency and $\mathrm{NH}_{3}$ slip as a function of average ANR for different UI. 148

Figure 5.27: $\mathrm{NH}_{3}$ storage as a function of average ANR for different UI 148

Figure 5.28: Single channel SCR model simulation results with calibration to reactor data compared to engine experimental data

Figure 5.29: Contour plot of the maldistribution factors applied to each channel of the 24channel model with parameters developed from the reactor data.

Figure 5.30: Multi-channel model simulation results compared to engine experimental data

Figure 5.31: Test protocol for SCR hydrothermal aging tests 154

Figure 5.32: Comparison of Cumulative $\mathrm{NH}_{3}$ stored between de-greened and aged catalysts

Figure 5.33: Axial $\mathrm{NO}_{\mathrm{x}}$ and $\mathrm{NH}_{3}$ distribution profiles at steps 4 and 7 under the temperature of $350^{\circ} \mathrm{C}$

Figure 5.34: $\mathrm{NH}_{3}$ storage as a function of temperature for de-greened and aged catalysts

Figure 5.35: Experimental and simulated cumulative $\mathrm{NH}_{3}$ stored under $\mathrm{NH}_{3}$ saturation condition for both de-greened and aged catalysts.

Figure 5.36: Arrhenius form plot of the $\mathrm{NH}_{3}$ oxidation reaction rate constants

Figure 5.37: Arrhenius form plot of the reaction rate constants for standard and fast SCR reactions

Figure B.1: DEF injector calibration curve

Figure F.1: Gaseous concentrations of $\mathrm{NO}, \mathrm{NO}_{2}$ and $\mathrm{NH}_{3}$ at the 0.6 of the catalyst length under temperature of $250^{\circ} \mathrm{C}$ 188 
Figure F.2: $\mathrm{NO}_{x}$ concentrations at equilibrium state of $\mathrm{NO}_{\mathrm{x}}+\mathrm{NH}_{3}$ and $\mathrm{NO}+\mathrm{NH}_{3}$ sections as a function of axial position for three temperatures 191

Figure F.3: $\mathrm{NO}_{x}$ concentrations at equilibrium state of $\mathrm{NO}_{\mathrm{x}}+\mathrm{NH} 3$ and $\mathrm{NO}+\mathrm{NH}_{3}$ sections as a function of axial position for three temperatures 191

Figure F.4: $\mathrm{NH}_{3}$ storage as a function of axial position for the temperature of $250^{\circ} \mathrm{C} \ldots 192$

Figure F.5: $\mathrm{NH}_{3}$ storage as a function of axial position for the temperature of $300^{\circ} \mathrm{C} \ldots 192$

Figure F.6: $\mathrm{NH}_{3}$ storage as a function of axial position for the temperature of $350^{\circ} \mathrm{C} \ldots 193$

Figure G.1: SCR inlet and outlet concentrations for $200^{\circ} \mathrm{C}$ and $60 \mathrm{k} \mathrm{hr}^{-1} \mathrm{SV}$ 194

Figure H.1: Comparison of SCR outlet $\mathrm{NH}_{3}$ between simulation and experimental results for the space velocity of $90,000 \mathrm{hr}^{-1}$ 196

Figure H.2: Simulated $\mathrm{NH}_{3}$ coverage fraction for TPD test for the space velocity of $90,000 \mathrm{hr}^{-1}$ 196

Figure H.3: Comparison of SCR outlet $\mathrm{NH}_{3}$ between simulation and experimental results for the space velocity of $120,000 \mathrm{hr}^{-1}$.... 197

Figure H.4: Simulated $\mathrm{NH}_{3}$ coverage fraction for TPD test for the space velocity of $120,000 \mathrm{hr}^{-1}$..... .197

Figure I.1: Model simulation vs. reactor data, SV $=60 \mathrm{k} / \mathrm{hr}$, Temp. $=200^{\circ} \mathrm{C}$ 198

Figure I.2: Model simulation vs. reactor data, $\mathrm{SV}=60 \mathrm{k} / \mathrm{hr}$, Temp. $=250^{\circ} \mathrm{C}$ 198

Figure I.3: Model simulation vs. reactor data, $\mathrm{SV}=60 \mathrm{k} / \mathrm{hr}$, Temp. $=300^{\circ} \mathrm{C}$ 199

Figure I.4: Model simulation vs. reactor data, $\mathrm{SV}=60 \mathrm{k} / \mathrm{hr}$, Temp. $=350^{\circ} \mathrm{C}$ 199

Figure I.5: Model simulation vs. reactor data, $S V=60 \mathrm{k} / \mathrm{hr}$, Temp. $=400^{\circ} \mathrm{C}$ 200

Figure I.6: Model simulation vs. reactor data, SV $=90 \mathrm{k} / \mathrm{hr}, \mathrm{Temp} .=200^{\circ} \mathrm{C}$ .200

Figure I.7: Model simulation vs. reactor data, $\mathrm{SV}=90 \mathrm{k} / \mathrm{hr}$, Temp. $=225^{\circ} \mathrm{C}$ 201

Figure I.8: Model simulation vs. reactor data, $\mathrm{SV}=90 \mathrm{k} / \mathrm{hr}$, Temp. $=250^{\circ} \mathrm{C}$ 201

Figure I.9: Model simulation vs. reactor data, $S V=90 \mathrm{k} / \mathrm{hr}$, Temp. $=275^{\circ} \mathrm{C}$ 202

Figure I.10: Model simulation vs. reactor data, $\mathrm{SV}=90 \mathrm{k} / \mathrm{hr}$, Temp. $=300^{\circ} \mathrm{C}$ .202

Figure I.11: Model simulation vs. reactor data, $\mathrm{SV}=90 \mathrm{k} / \mathrm{hr}$, Temp. $=325^{\circ} \mathrm{C}$ .203

Figure I.12: Model simulation vs. reactor data, SV $=90 \mathrm{k} / \mathrm{hr}$, Temp. $=350^{\circ} \mathrm{C}$ .203

Figure I.13: Model simulation vs. reactor data, $\mathrm{SV}=90 \mathrm{k} / \mathrm{hr}$, Temp. $=375^{\circ} \mathrm{C} \ldots \ldots \ldots \ldots . . .204$

Figure I.14: Model simulation vs. reactor data, $\mathrm{SV}=90 \mathrm{k} / \mathrm{hr}$, Temp. $=400^{\circ} \mathrm{C} \ldots \ldots \ldots \ldots . . .204$

Figure I.15: Model simulation vs. reactor data, $\mathrm{SV}=90 \mathrm{k} / \mathrm{hr}$, Temp. $=425^{\circ} \mathrm{C} \ldots \ldots \ldots \ldots .205$

Figure I.16: Model simulation vs. reactor data, SV $=120 \mathrm{k} / \mathrm{hr}$, Temp. $=200^{\circ} \mathrm{C}$ .205 
Figure I.17: Model simulation vs. reactor data, SV $=120 \mathrm{k} / \mathrm{hr}, \mathrm{Temp} .=250^{\circ} \mathrm{C} \ldots \ldots \ldots . . .206$

Figure I.18: Model simulation vs. reactor data, SV $=120 \mathrm{k} / \mathrm{hr}$, Temp. $=300^{\circ} \mathrm{C} \ldots \ldots \ldots . .206$

Figure I.19: Model simulation vs. reactor data, SV $=120 \mathrm{k} / \mathrm{hr}$, Temp. $=350^{\circ} \mathrm{C} \ldots \ldots \ldots . .207$

Figure I.20: Model simulation vs. reactor data, SV $=120 \mathrm{k} / \mathrm{hr}$, Temp. $=400^{\circ} \mathrm{C} \ldots \ldots \ldots . . .207$

Figure J.1: Model simulation compared to steady state engine data for test point 1 with the SCR inlet temperature of $400^{\circ} \mathrm{C}$ 208

Figure J.2: Model simulation compared to steady state engine data for test point 3 with the SCR inlet temperature of $353^{\circ} \mathrm{C}$ 208

Figure J.3: Model simulation compared to steady state engine data for test point 4 with the SCR inlet temperature of $331^{\circ} \mathrm{C}$ 209

Figure J.4: Model simulation compared to steady state engine data for test point 5 with the SCR inlet temperature of $301^{\circ} \mathrm{C}$ 209

Figure J.5: Model simulation compared to steady state engine data for test point 6 with the SCR inlet temperature of $278^{\circ} \mathrm{C}$ 210

Figure J.6: Model simulation compared to steady state engine data for test point 7 with the SCR inlet temperature of $252^{\circ} \mathrm{C}$ 210

Figure J.7: Model simulation compared to steady state engine data for the repeat of test point 7 with the SCR inlet temperature of $252^{\circ} \mathrm{C}$

Figure J.8: Model simulation compared to steady state engine data for the repeat of test point 4 with the SCR inlet temperature of $330^{\circ} \mathrm{C}$.

Figure K.1: Simulated concentrations compared to experimental measurements of transient experiment 2

Figure K.2: Simulated cumulative $\mathrm{NO}_{x}, \mathrm{NH}_{3}$ and $\mathrm{NO}_{\mathrm{x}}$ conversion efficiency of transient experiment 2 .

Figure K.3: Simulated concentrations compared to experimental measurements of transient experiment 3

Figure K.4: Simulated cumulative $\mathrm{NO}_{x}, \mathrm{NH}_{3}$ and $\mathrm{NO}_{\mathrm{x}}$ conversion efficiency of transient experiment 3 .

Figure K.5: Simulated concentrations compared to experimental measurements of transient experiment 4

Figure K.6: Simulated cumulative $\mathrm{NO}_{\mathrm{x}}, \mathrm{NH}_{3}$ and $\mathrm{NO}_{\mathrm{x}}$ conversion efficiency of transient experiment 4 214 
Figure K.7: Simulated concentrations compared to experimental measurements of transient experiment 5 ...

Figure K.8: Simulated cumulative $\mathrm{NO}_{x}, \mathrm{NH}_{3}$ and $\mathrm{NO}_{\mathrm{x}}$ conversion efficiency of transient experiment 5

Figure K.9: Simulated concentrations compared to experimental measurements of transient experiment 6 . 216

Figure K.10: Simulated cumulative $\mathrm{NO}_{x}, \mathrm{NH}_{3}$ and $\mathrm{NO}_{\mathrm{x}}$ conversion efficiency of transient experiment 6 . 216

Figure L.1: Distribution profile of the SCR inlet ANR for test point 1 .......................217

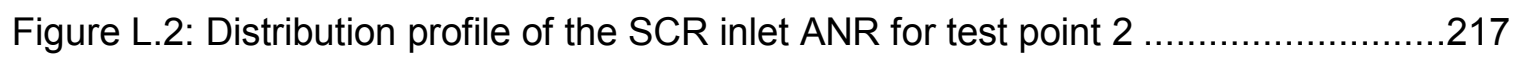

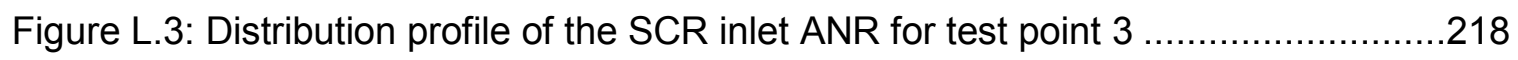

Figure L.4: Distribution profile of the SCR inlet ANR for test point 4 ........................218

Figure L.5: Distribution profile of the SCR inlet ANR for test point 5 .......................219

Figure L.6: Distribution profile of the SCR inlet ANR for test point 6 .......................219 


\section{List of Tables}

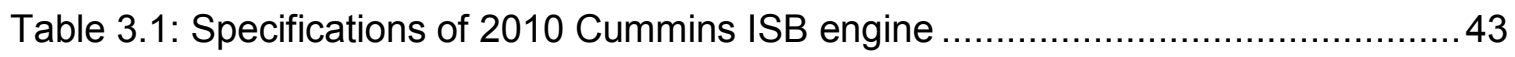

Table 3.2: Fuel properties for the ULSD used for SCR testing ................................. 44

Table 3.3: Specifications of the catalysts in 2010 Cummins ISB engine aftertreatment system. .45

Table 3.4: Engine operating points for de-greening the aftertreatment system. 45

Table 3.5: Specifications of thermocouples used in ISB aftertreatment system .50

Table 3.6: Specification of differential pressure transducers. 51

Table 3.7: Monitoring mass and the ion source for each measured species. .53

Table 3.8: Specifications of calibration gases for IMR-MS 53

Table 3.9: Specifications of the $\mathrm{NO}_{x}$ sensor and $\mathrm{NH}_{3}$ sensor. .54

Table 3.10: Description of the data acquisition system. .55

Table 3.11: Engine speed/load points for SCR tests ............................................57

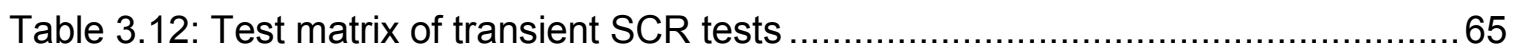

Table 3.13: Test matrix for the $\mathrm{NH}_{3}$ maldistribution test.........................................72

Table 3.14 Details of the core SCR sample.........................................................

Table 3.15: Gas species concentrations for each test step of the reactor test protocol.. 75

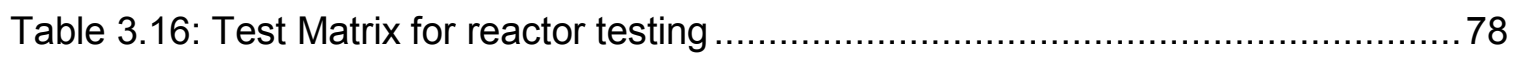

Table 3.17: Details of each step of the Spaci-IR test protocol ................................... 80

Table 3.18: Test matrix of the Spaci-IR tests. .80

Table 4.1: Reactions included in the model and reaction rate calculation for each reaction 87

Table 4.2: Identified $\mathrm{NH}_{3}$ storage capacity, Aads/Ades and Eads-Edes for 1-site model.. 91

Table 4.3: Identified $\mathrm{NH}_{3}$ storage capacity, Aads/Ades and Eads-Edes for the storage sites of the 2-site model. .96

Table 4.4: Identified $\mathrm{NH}_{3}$ storage parameters. 103

Table 4.5: Identified $\mathrm{NH}_{3}$ oxidation parameters 105

Table 4.6: Identified model parameters for standard SCR reactions and NO oxidation reaction 106 
Table 4.7: Identified model parameters for slow SCR reactions and $\mathrm{N}_{2} \mathrm{O}$ formation reaction ................................................................................ 107

Table 4.8: Identified model parameters for fast SCR reaction ..................................108

Table 4.9: SCR model calibration results based on the reactor data........................109

Table 4.10: Optimized model parameters of each temperature run for the SCR model based on the engine data........................................................... 115

Table 4.11: SCR model calibration results based on the engine data ......................116

Table 5.1: SCR model parameters of the calibrated SCR model along with literature values

Table 5.2: Cost function values for each gas species from comparison of SCR outlet concentrations between model simulation and experimental measurements

Table 5.3: Cost function values for each gas species of the model simulation with identified model parameter.

Table 5.4: Cost function values of model simulation compared to experimental measurements for transient tests 134

Table 5.5: Simulation results of $\mathrm{NO}_{2} / \mathrm{NO}_{x}$ ratio effects .138

Table 5.6: Test results for the $\mathrm{NH}_{3}$ maldistribution tests .145

Table 5.7: Simulation conditions for maldistribution effect study 147

Table 5.8: Cost values for simulation results from the single and multi-channel models

Table 5.9: Details for each step of the Spaci-IR aging test protocol ..........................154

Table 5.10: Details for each step of the Spaci-IR aging test protocol .........................158

Table 5.11: Kinetic parameters for $\mathrm{NH}_{3}$ oxidation reaction .................................160

Table 5.12: Kinetic parameters for the standard and fast SCR reactions .....................161

Table E.1: $\mathrm{NH}_{3}$ sensor calibration results ..................................................187

Table G.1: Reactor experimental data sets with marked steps $(\times)$ that were not used for model calibration. .195 


\section{Acknowledgements}

I would like to take this opportunity to thank every individual or institution that supported, assisted and helped me in any way to complete this thesis.

Foremost, I would like to express my deepest appreciation to my advisors, Dr. John Johnson and Dr. Jeffrey Naber for providing the opportunity and the continuous support for me to pursue my $\mathrm{PhD}$ degree. Without their persistent guidance and help this thesis would not have been possible. I would like to thank Dr. Gordon Parker for his engagement and inputs through the research process. I would like to thank Dr. Jason Keith and Dr. Gordon Parker for being on my research committee.

I would like to thank the past and present team members in the Heavy Duty Diesel testing and modeling groups. Their assistance and help made it possible for me to complete the experimental and modeling work. I would like to thank Josh Pihl at Oak Ridge National Laboratory for providing and explaining the reactor data. I would like to thank the industry partners Cummins, John Deere and Johnson Matthey for evaluating the SCR model and giving helpful comments to make improvement. I would also like to thank the U.S. Department of Energy for the financial support under award number of DE-EE0000204. Thanks to John Deere for providing part of the financial support during the last semester of my thesis research.

Last but not least, I would like thank my friends and family for their unconditional support and encouragement. Words cannot express my gratitude.

This material was prepared as an account of work sponsored by an agency of the United States Government. Neither the United States Government nor any agency thereof, nor any of their employees, makes any warranty, express or implied, or assumes any legal liability or responsibility for the accuracy, completeness, or usefulness of any information, apparatus, product, or process disclosed, or represents that its use would not infringe privately owned rights. Reference herein to any specific commercial product, 
process, or service by trade name, trademark, manufacturer, or otherwise does not necessarily constitute or imply its endorsement, recommendation, or favoring by the United States Government or any agency thereof. The views and opinions of authors expressed herein do not necessarily state or reflect those of the United States Government or any agency thereof. 


\section{Abstract}

$\mathrm{A} \mathrm{NO}_{\mathrm{x}}$ reduction efficiency higher than $95 \%$ with $\mathrm{NH}_{3}$ slip less than $30 \mathrm{ppm}$ is desirable for heavy-duty diesel (HDD) engines using selective catalytic reduction (SCR) systems to meet the US EPA $2010 \mathrm{NO}_{x}$ standard and the 2014-2018 fuel consumption regulation. The SCR performance needs to be improved through experimental and modeling studies. In this research, a high fidelity global kinetic 1-dimensional 2-site SCR model with mass transfer, heat transfer and global reaction mechanisms was developed for a Cu-zeolite catalyst. The model simulates the SCR performance for the engine exhaust conditions with $\mathrm{NH}_{3}$ maldistribution and aging effects, and the details are presented.

SCR experimental data were collected for the model development, calibration and validation from a reactor at Oak Ridge National Laboratory (ORNL) and an engine experimental setup at Michigan Technological University (MTU) with a Cummins 2010 ISB engine. The model was calibrated separately to the reactor and engine data. The experimental setup, test procedures including a surrogate HD-FTP cycle developed for transient studies and the model calibration process are described. Differences in the model parameters were determined between the calibrations developed from the reactor and the engine data. It was determined that the SCR inlet $\mathrm{NH}_{3}$ maldistribution is one of the reasons causing the differences. The model calibrated to the engine data served as a basis for developing a reduced order SCR estimator model.

The effect of the SCR inlet $\mathrm{NO}_{2} / \mathrm{NO}_{\mathrm{x}}$ ratio on the SCR performance was studied through simulations using the surrogate HD-FTP cycle. The cumulative outlet $\mathrm{NO}_{\mathrm{x}}$ and the overall $\mathrm{NO}_{x}$ conversion efficiency of the cycle are highest with a $\mathrm{NO}_{2} / \mathrm{NO}_{\mathrm{x}}$ ratio of 0.5 . The outlet $\mathrm{NH}_{3}$ is lowest for the $\mathrm{NO}_{2} / \mathrm{NO}_{x}$ ratio greater than 0.6 .

A combined engine experimental and simulation study was performed to quantify the $\mathrm{NH}_{3}$ maldistribution at the SCR inlet and its effects on the SCR performance and kinetics. The uniformity index (UI) of the SCR inlet $\mathrm{NH}_{3}$ and $\mathrm{NH}_{3} / \mathrm{NO}_{x}$ ratio (ANR) was determined to be below 0.8 for the production system. The UI was improved to 0.9 after 
installation of a swirl mixer into the SCR inlet cone. A multi-channel model was developed to simulate the maldistribution effects. The results showed that reducing the $\mathrm{UI}$ of the inlet ANR from 1.0 to 0.7 caused a $5-10 \%$ decrease in $\mathrm{NO}_{\mathrm{x}}$ reduction efficiency and $10-20 \mathrm{ppm}$ increase in the $\mathrm{NH}_{3}$ slip. The simulations of the steady-state engine data with the multi-channel model showed that the $\mathrm{NH}_{3}$ maldistribution is a factor causing the differences in the calibrations developed from the engine and the reactor data.

The Reactor experiments were performed at ORNL using a Spaci-IR technique to study the thermal aging effects. The test results showed that the thermal aging (at $800^{\circ} \mathrm{C}$ for 16 hours) caused a $30 \%$ reduction in the $\mathrm{NH}_{3}$ stored on the catalyst under $\mathrm{NH}_{3}$ saturation conditions and different axial concentration profiles under SCR reaction conditions. The kinetics analysis showed that the thermal aging caused a reduction in total $\mathrm{NH}_{3}$ storage capacity $\left(94.6\right.$ compared to $138 \mathrm{gmol} / \mathrm{m}^{3}$ ), different $\mathrm{NH}_{3}$ adsorption/desorption properties and a decrease in activation energy and the preexponential factor for $\mathrm{NH}_{3}$ oxidation, standard and fast SCR reactions. Both reduction in the storage capability and the change in kinetics of the major reactions contributed to the change in the axial storage and concentration profiles observed from the experiments. 



\section{Chapter 1.}

\section{Introduction}

\subsection{Background}

The diesel engine is known for its thermal efficiency, durability, and reliability. It is the main power source for medium and heavy-duty on-road vehicles including trucks, buses, off-road vehicles and industrial equipment. Diesel engines are also used as a power source for passenger cars and light-duty trucks particularly in Europe. The annual diesel fuel consumption in the United States is more than 40 billion gallons and up to $93 \%$ of the diesel is consumed by on road heavy-duty diesel (HDD) engines [1]. The energy demand for heavy trucks is predicted to increase by $20 \%$ in 2035 compared to 2010 [2].

Despite the advantages and the popularity of the diesel engine, diesel emissions are considered as adverse to human health and the atmosphere. Diesel combustion products include carbon monoxide (CO), unburned hydrocarbons ( $\mathrm{HC})$, carbon dioxide $\left(\mathrm{CO}_{2}\right)$ which is a greenhouse gas, nitrogen oxides $\left(\mathrm{NO}_{\mathrm{x}}\right)$ which is the sum of nitric oxide (NO) and nitrogen dioxide $\left(\mathrm{NO}_{2}\right)$, and particulate matter (PM). The diesel fuel currently used in the U.S. contains less than 15 parts per million (ppm) of sulfur, low levels of sulfur oxidation products ( $<1 \mathrm{ppm})$ including sulfur dioxide $\left(\mathrm{SO}_{2}\right)$ and sulfur trioxide $\left(\mathrm{SO}_{3}\right)$ are thus emitted from the diesel engine.

Starting in 1974, the U.S. Environmental Protection Agency (EPA) and other organizations began to develop regulations for diesel engine emissions [3]. Those standards and regulations have become more stringent in the past 30 years. An evolution of regulations for HDD engines is shown in Figure 1.1. The U.S. EPA on-Road HDD regulations for 2010 were 0.2 and $0.01 \mathrm{~g} / \mathrm{bhp} \cdot \mathrm{hr}$ for $\mathrm{NO}_{\mathrm{x}}$ and $\mathrm{PM}$ respectively, which 
are a magnitude lower than the EPA 1998 regulations. The European emission standard shows the similar trend. From EURO III regulation which was in effect in 2000 to EURO VI which will be in effect in 2013, the $\mathrm{NO}_{\mathrm{x}}$ and PM limits are reduced from 3.8 and 0.075 $\mathrm{g} / \mathrm{bhp} \cdot \mathrm{hr}$ to 0.3 and $0.0075 \mathrm{~g} / \mathrm{bhp} \cdot \mathrm{hr}$ respectively. The upcoming 2014-2018 fuel consumption standards [4] necessitate the optimization of passive oxidation of PM in order to reduce the frequency of active regenerations, thus reducing the fuel consumption in additional to the engine changes. At the same time, extensive research has been carried out to reduce diesel emission levels by more than a magnitude to meet the regulations [5-13].

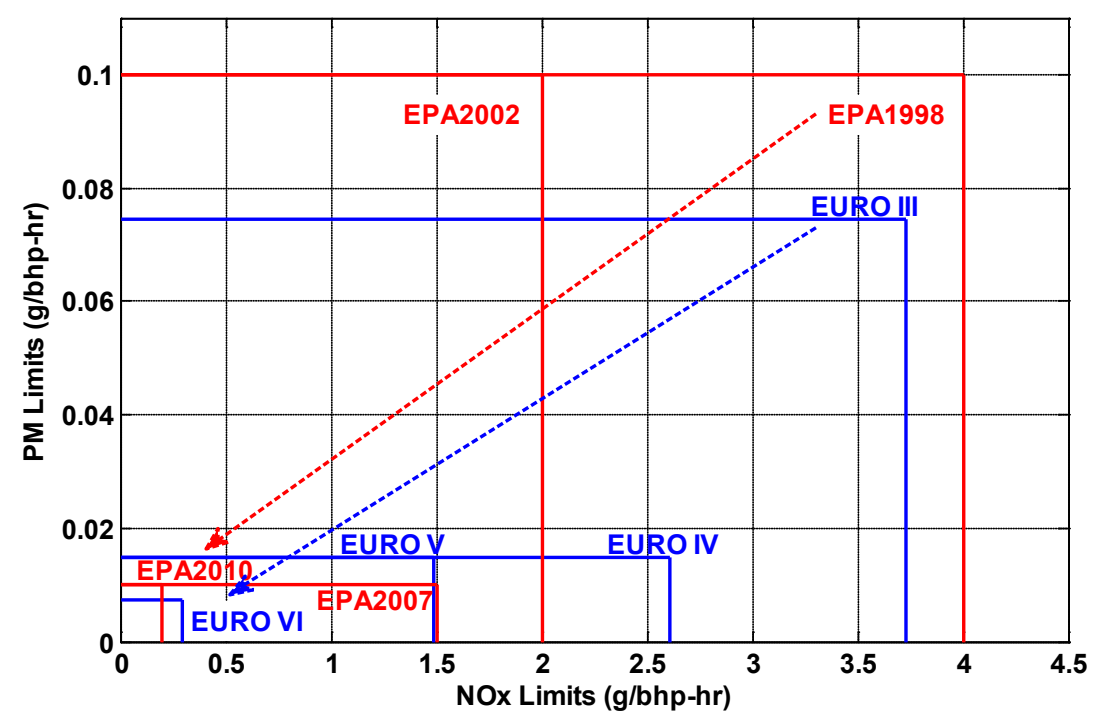

Figure 1.1: An evolution of U.S. EPA and Europe regulations for HDD engines [14]

\subsection{HDD Aftertreatment Systems}

It has not been possible to reduce emissions to meet the EURO V and EPA 2010 regulations by only improving the engine technologies. Therefore, aftertreatment systems have been developed to reduce $\mathrm{PM}$ and $\mathrm{NO}_{\mathrm{x}}$ to meet the regulations $[8,9]$. $\mathrm{A}$ typical aftertreatment system for HDD engines for EURO $V$ and EPA 2010 is shown in Figure 1.2. The system consists of a diesel oxidation catalyst (DOC), catalyzed 
particulate filter (CPF), selective catalytic reduction (SCR) system with urea injection, and an ammonia oxidation catalyst (AMOX).

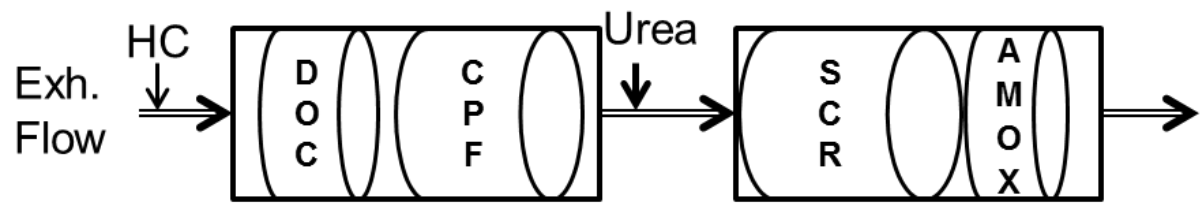

Figure 1.2: Typical layout of HDD engine aftertreatment system

The DOC is a flow through device with square channels open at both ends with a honeycomb structure. The DOC is used for oxidizing $\mathrm{CO}$ and $\mathrm{HC}$, and converting $\mathrm{NO}$ to $\mathrm{NO}_{2}$. The $\mathrm{NO}_{\mathrm{x}}$ in the diesel engine out exhaust typically contains less than $10 \%$ of $\mathrm{NO}_{2}$ [15]. Oxidation of $\mathrm{NO}$ to $\mathrm{NO}_{2}$ through the DOC provides additional $\mathrm{NO}_{2}$ for passively regenerating the CPF and for providing optimal $\mathrm{NO}_{2} / \mathrm{NO}_{x}$ ratio for better SCR $\mathrm{NO}_{x}$ reduction efficiency [16]. This will be discussed in detail in later sections. The CO conversion efficiency across the DOC can approach 100\% for normal engine operating conditions. The $\mathrm{HC}$ and $\mathrm{NO}$ conversion efficiencies across the DOC are functions of temperature and space velocity. Typically the $\mathrm{HC}$ conversion efficiency increases with increased temperature. The $\mathrm{NO}$ to $\mathrm{NO}_{2}$ conversion efficiency is dependent upon the catalyst formation, temperature and operating space velocity. A maximum $\mathrm{NO}$ to $\mathrm{NO}_{2}$ conversion efficiency of about $65 \%$ occurs at the temperature of $325^{\circ} \mathrm{C}$.

The CPF is a filter that collects PM contained in the exhaust in and on the substrate wall. It is a wall flow device with every other channel open in the inlet face but each open inlet face channel is closed in the outlet face. Therefore, the exhaust going into the inlet channel has to pass through the porous substrate wall to get to the outlet channel and exit the filter. The filtration efficiency of the CPF can reach $97 \%$ once a PM cake layer forms on the substrate wall. As PM is being loaded in and on the wall, the pressure drop across the CPF increases thus increasing the back pressure of the engine which results in lower efficiency and higher fuel consumption [17]. The $\mathrm{NO}_{2}$ generated by the $\mathrm{DOC}$ from oxidizing $\mathrm{NO}$ can react with PM in the CPF at relatively low temperatures $\left(>250^{\circ} \mathrm{C}\right)$ to reduce the pressure drop across the CPF. This process is known as $\mathrm{NO}_{2}$ assisted 
oxidation. Above $400^{\circ} \mathrm{C}$, thermal or $\mathrm{O}_{2}$ oxidation also occurs at a significant rate. When there is no external energy source, this process of oxidation is called "passive oxidation". However, this method is not always effective because of the temperature and $\mathrm{PM}$ to $\mathrm{NO}_{\mathrm{x}}$ ratio is not sufficient to oxidize PM by passive oxidation. In order to effectively remove the PM retained in the CPF and maintain the pressure drop at low levels, an active regeneration process by injection of diesel fuel either late in the cycle (in-cylinder or in the exhaust)t is carried out. The fuel is oxidized in the DOC, and this generates an increase in temperature which with excess $\mathrm{O}_{2}$ in the exhaust gas oxidizes the PM retained in the CPF.

The urea SCR system is the leading technology for $\mathrm{NO}_{x}$ reduction in $\mathrm{HDD}$ engines which uses a urea-water solution as reductant to reduce $\mathrm{NO}_{x}$ on the SCR catalyst surface. A urea water solution with urea concentration of $32.5 \%$ by weigh is used as the reductant for automotive applications and HDD applications in the US. This solution goes by different names including diesel exhaust fluid (DEF) in the U.S. or Ad-Blue in Europe. The DEF is introduced to the exhaust stream by an injector located before the SCR bricks (see Figure 1.2). Typically there is a mixing tube between the injector and SCR bricks to help mix the urea with the exhaust flow and accelerate the urea hydrolysis and decomposition process. The desired urea decomposition product is ammonia $\left(\mathrm{NH}_{3}\right)$, which will be adsorbed on the catalyst surface, and the stored $\mathrm{NH}_{3}$ reacts with $\mathrm{NO}_{x}$ on the catalyst surface through a number of pathways to form $\mathrm{H}_{2} \mathrm{O}$ and $\mathrm{N}_{2}$. This stored ammonia can also be desorbed at high temperature or oxidized with oxygen. Anhydrous ammonia can also be used as a reductant without going through a hydrolysis or decomposition process, but compared to the urea-water solution, it is extremely toxic and difficult to store.

The SCR substrate or brick is a flow through device with square channels open at both ends with a typical channel density of 400 cells per square inch (CPSI). This honeycomb like structure is the catalyst carrier made from various ceramic materials including titanium oxide and cordierite. The channels are covered with active catalytic components. Those components are typically oxides of base metals including vanadium and tungsten, zeolites, and precious metals $[18,19]$. Oxides of vanadium and tungsten 
are usually less expensive and perform well under temperatures below $500{ }^{\circ} \mathrm{C}$. However, their thermal durability is a problem for applications with temperatures above $500^{\circ} \mathrm{C}$ generated by the active regeneration of the CPF [20]. Zeolite catalysts have better low temperature performance and the capability of remaining active at high temperatures of $600^{\circ} \mathrm{C}$ and transient temperatures of up to $850^{\circ} \mathrm{C}$ [21]. Iron (Fe) and copper $(\mathrm{Cu})$ zeolite catalysts are two popular choices for SCR systems installed downstream of the CPF [10]. The performance of the catalysts will be described in detail in the next chapter.

A typical aftertreatment system also consists of an ammonia oxidation catalyst (AMOX) after the SCR. For the urea SCR, under situations of over injecting urea, low exhaust temperature, and with the SCR being aged, ammonia slip (ammonia going through the SCR without being fully converted) will be present at the SCR outlet. Ammonia is harmful to human health and the environment and is usually required to be below 10-30 ppm. The AMOX is commonly placed after the SCR bricks to oxidize ammonia to $\mathrm{N}_{2}$ and $\mathrm{H}_{2} \mathrm{O}$.

Other ways to reduce $\mathrm{NO}_{x}$ emissions include engine based exhaust gas recirculation (EGR), and aftertreatment based lean $\mathrm{NO}_{\mathrm{x}}$ traps (LNT). EGR works by recirculation of a certain fraction of the exhaust gas back into the cylinders. This lowers the adiabatic flame temperature thus reducing the level of thermal formation of $\mathrm{NO}_{x}$. All diesel engines now incorporate the EGR technology. The $\mathrm{NO}_{\mathrm{x}}$ reduction efficiency higher than $95 \%$ is highly desirable for highway heavy-duty applications to meet the EPA $2010 \mathrm{NO}_{\mathrm{x}}$ regulations [12]. However, using only EGR is not sufficient to meet the EPA $2010 \mathrm{NO}_{\mathrm{x}}$ regulation and a higher level of EGR increases the PM concentration in the exhaust so that fuel consumption will be increased because more fuel needs to be used for CPF active regeneration. $\mathrm{LNT}$ is $\mathrm{HC}$ based $\mathrm{NO}_{\mathrm{x}}$ reduction method typically used on light-duty applications. LNT works by trapping $\mathrm{NO}_{x}$ on the zeolite surface. When the storage of the trap is full, a rich regeneration process is run by introducing $\mathrm{HC}$ into exhaust to react with the trapped $\mathrm{NO}_{x}$ and produce $\mathrm{H}_{2} \mathrm{O}$ and $\mathrm{N}_{2}[10]$. 


\subsection{Motivation}

Effective $\mathrm{NO}_{x}$ control for the HDD engine is becoming more important to meet the fuel consumption and emission regulations. It requires high $\mathrm{NO}_{x}$ reduction efficiency $(>90 \%)$, and low ammonia slip ( $<30 \mathrm{ppm}$ ) under a wide range of operating conditions for the SCR system. Understanding the SCR characteristics is becoming more imperative for system improvement. Well-designed SCR experiments are necessary to characterize the SCR performance and the effects of different variables including temperature, space velocity and inlet gas composition. However, the SCR system is a complex non-linear system in which there is limited ability to measure the internal states directly. In order to improve the understanding of the SCR system and the chemical kinetics, extensive experimental studies along with modeling efforts are required. An SCR model calibrated to experimental data provides possibilities to estimate the SCR states which cannot be directly measured.

Furthermore, it is time intensive and costly to use the conventional experimental approach to develop the SCR system because there are a significant number of variables that need to be considered when designing and optimizing the system. These variables include dimensions, wall thickness, cell density of the catalyst, fuel and urea injection control strategies, etc. Changes in each variable can cause differences in the overall system performance. However, running an accurate simulation model in conjunction with the experimental approach allows simulating a wider range of different scenarios, which then takes less time and is more cost effective. As a result, a high fidelity SCR model developed based on experimental studies is able to simulate a wide range of scenarios in a timely and cost efficient way.

A well developed and accurately calibrated SCR model is able to predict the SCR internal states which cannot be directly or easily measured. Those states include the gaseous concentrations at different axial locations of the catalyst as well as the $\mathrm{NH}_{3}$ stored on the catalyst. The SCR performance is highly dependent on the $\mathrm{NH}_{3}$ coverage on the catalyst surface. For the application of the SCR on diesel engines, the system is exposed to highly transient conditions. This makes the SCR difficult to reach steady 
state operating conditions. As a result, the ability of a SCR model to correctly predict the internal states under transient conditions is important to SCR state estimation and SCR model based control strategies. A high fidelity SCR model calibrated to engine data can serve as a basis for further developing a SCR estimator model that estimates the SCR states in real time [22]. This is meaningful for developing the OBD system and model based control strategies for on-vehicle applications.

Extensive SCR data are required to develop and calibrate the SCR model. Testing the SCR in a flow reactor is an efficient first step in studying the basic characteristics of the catalyst. Simulated exhaust flow generated from different gas cylinders are used in SCR reactor tests and gaseous $\mathrm{NH}_{3}$ is used as reductant. The test conditions including gas flow rate, temperature, and SCR inlet gaseous concentrations can be precisely controlled. With carefully designed test protocols and procedures, the SCR reactor tests provide opportunities for characterization of the SCR performance and analyzing the reactions taking place as well as the kinetic parameters involved in the reactions.

However, the flow reactor test environment is much simpler than the real SCR exposure conditions in the engine aftertreatment system. The reactor studies provide accurate performance characterization and kinetic analysis of the SCR catalyst but do not account for the more complex conditions (complex urea decomposition process, maldistribution of $\mathrm{NH}_{3}$.etc.) which could occur in engine applications. As a result, well-designed SCR engine tests and a model calibrated to the engine experimental data are important for developing models for vehicle applications. The model should be able to make reliable predictions for both steady-state and transient engine operating conditions. It is also important to understand the factors that affect the SCR performance in the engine aftertreatment system.

\subsection{Goals and Objectives}

The goal of this research is to develop, validate and apply a high fidelity SCR model applicable to simulate the SCR in the engine conditions. To achieve this goal, coupled 
experimental and modeling studies were carried out to determine the critical kinetic parameters controlling the dynamics of the SCR system performance under varying temperatures, space velocities, exhaust inlet species, inlet ammonia maldistribution levels and aging conditions with controlled DEF injection for a Cu-zeolite SCR catalyst and to quantify the variable impacts on ammonia storage and $\mathrm{NO}_{\mathrm{x}}$ conversion. The specific objectives developed to meet the research goal are as follows:

1. Develop an engine test cell setup with instruments and a data acquisition system for both steady state and transient aftertreatment system testing

2. Develop a Cu-zeolite SCR model from the model originally developed for Fe-zeolite reactor data [23] by modifying the mass transfer correlations and the global reaction mechanisms to simulate the Cu-zeolite experimental data

3. Develop the SCR engine test procedures and conduct SCR engine experiments to collect both steady-state and transient data for the purpose of calibrating the SCR model and validating the model performance

4. Process the SCR reactor experimental data to characterize the SCR performance and determine the internal SCR states under different space velocities and temperatures. Use the data to calibrate the high fidelity SCR model by determining the model storage capacities and the critical reaction kinetic parameters

5. Use the SCR engine experimental data to calibrate the SCR model by determining the $\mathrm{NH}_{3}$ storage parameters and the reaction kinetic parameters. The SCR model parameters identified from the reactor data based are used as a starting point for the SCR model calibration with engine data. Validate the model performance against SCR transient engine experimental data. Compare the SCR models separately calibrated to reactor and engine experimental data

6. Characterize $\mathrm{NH}_{3}$ maldistribution profiles with two mixers, e.g. a production and a swirl mixer at the SCR inlet, through experimental studies and data analysis. 
Determine the $\mathrm{NH}_{3}$ maldistribution effects on the SCR performance in terms of $\mathrm{NO}_{x}$ conversion, $\mathrm{NH}_{3}$ slip and $\mathrm{NH}_{3}$ storage. Quantify the ammonia maldistribution effect on the SCR kinetic parameters

7. Determine the hydrothermal aging effects on the SCR performance and kinetics from Spaci-IR data.

\subsection{Dissertation Outline}

This chapter presented a brief background of the research. The increasingly stringent emission and fuel consumption regulations as well as the typical diesel engine aftertreatment systems to meet the regulations were introduced. This was followed by a description of the research motivation, research goals and objectives.

Chapter 2 provides a literature review of the SCR system development as well as the significant studies related to this thesis. Information about SCR characteristics, experimental and modeling studies from the published works are summarized and presented. The thesis research findings from the experimental and modeling advances are presented in Chapters 4 and 5 and they are compared to the published works.

Chapter 3 provides detailed information about the test cell setup and test procedures for collecting SCR data. The engine test cell will be introduced with information about the engine, dynamometer, aftertreatment system, and specific instruments to collect temperature, pressure drop, flow rates, and emissions data. The test procedures and test matrix of the SCR engine experiments including steady state, transient, and $\mathrm{NH}_{3}$ maldistribution tests are given in this chapter. The reactor test setup along with the test protocols developed at ORNL and test conditions for both ordinary and space-IR reactor tests are also introduced.

Chapter 4 describes the 1D high fidelity SCR model and the model calibration procedures. The SCR model equations for mass transfer, heat transfer, storage 
equation, reaction mechanism and the reaction rate calculations are introduced to give the detailed description of the model. Procedures for calibrating the model separately to reactor data sets and engine steady state data are also presented in this chapter.

Chapter 5 presents the model calibration results as well as the performance of the calibrated model. The model calibrated to the reactor and engine data are validated by comparing the simulation results with the experimental measurements. The differences in the model parameters identified from the reactor and engine data are presented and the reason for the differences will be explained. The experimental and modeling studies of the hydrothermal aging effects on the SCR performance and kinetics are presented.

Chapter 6 is a summary of the accomplishments of this research, and the conclusions from the experimental and modeling studies. Recommendations about the future research directions are presented. 


\section{Chapter 2.}

\section{Literature Review}

A literature review of the aspects related to the SCR studies including experimental and modeling efforts from published research are presented in this chapter. A discussion of the advances of this thesis related to the past accomplishments is presented.

\subsection{Emission Control Technologies}

As discussed in the Introduction, the diesel emission regulations require research and development in emission control technologies. Diesel industry has had to meet the transient cycle emission standards since 1988 [24]. From 1988 to 2002, the diesel emission standards for $\mathrm{NO}_{x}$ and $\mathrm{PM}$ were met mainly by improving the engine technologies including retarding injection timing, better engine breathing design, improved electronic engine controls, increased fuel injection pressure, higher fuel quality, etc. Some engines in the 1990s used a DOC to reduce the PM concentration to meet the standards.

The EPA 2002 regulations reduced the $\mathrm{NO}_{\mathrm{x}}$ by $50 \%$, from 4.0 to $2.0 \mathrm{~g} / \mathrm{bhp} \cdot \mathrm{hr}$ while the $\mathrm{PM}$ standard was maintained at $0.1 \mathrm{~g} / \mathrm{bhp} \cdot \mathrm{hr}$. The $\mathrm{NO}_{\mathrm{x}}$ emission standards was met via application of exhaust gas recirculation (EGR) which was widely used for gasoline and light-duty and heavy-duty diesel engines $[25,26]$. EGR works by recirculating a fraction of exhaust gas back into the cylinder. This lowers the in-cylinder flame temperature thus reducing the level of $\mathrm{NO}_{x}$ thermal formation. However, the application of EGR on heavyduty diesel engines sacrificed the fuel consumption, reduced the engine durability and increased the PM emissions. The trade-off effects between the $\mathrm{NO}_{\mathrm{x}}$ and $\mathrm{PM}$ emissions 
[27] made it challenging to significantly reduce both emissions through engine technologies to meet the EPA 2007 and 2010 standards.

Research on the aftertreatment technologies including the PM filter and catalytic $\mathrm{NO}_{\mathrm{x}}$ reduction increased. An integrated aftertreatment system with DOC, DPF and SCR was investigated. The combined application of a DPF and a SCR on a heavy-duty diesel engine was reported to realize higher than 95\% PM filtration efficiency and $85 \% \mathrm{NO}_{\mathrm{x}}$ reduction on the European Stationary Cycle test procedure (ESC) cycle [24].

The PM standard was further reduced from the EPA 2002 regulations by $90 \%$ and the $\mathrm{NO}_{\mathrm{x}}$ by $25 \%$ for the EPA 2007 regulations. The $\mathrm{NO}_{\mathrm{x}}$ and PM limits became 1.5 and 0.01 $\mathrm{g} / \mathrm{bhp} \cdot \mathrm{hr}$ respectively. A diesel particulate filter (DPF) along with cooled EGR technology became the dominant solution for the heavy-duty diesel engines to meet the 2007 emission regulations [8]. A DOC is typically installed before the DPF to oxidize $\mathrm{CO}, \mathrm{HC}$, $\mathrm{NO}$ to $\mathrm{NO}_{2}$ and the diesel fuel injected into the exhaust stream to thermally oxidize the PM retained in the DPF. The SCR was not required to meet the EPA 2007 regulations, but its advantages and the potential of higher $\mathrm{NO}_{\mathrm{x}}$ conversion efficiency had been proved and made it a promising technology to meet the 2010 emission standards.

The SCR became widely used by the diesel industry in the 2010 to meet the EPA 2010 regulations [28]. The $\mathrm{NO}_{\mathrm{x}}$ and $\mathrm{PM}$ limits were set to 0.02 and $0.01 \mathrm{~g} / \mathrm{bhp} \cdot \mathrm{hr}$ respectively. The $\mathrm{NO}_{\mathrm{x}}$ standard was met by the combination of SCR and cooled EGR while the PM was reduced by the catalyzed particulate filter (CPF). A DOC was installed before the CPF to oxidize $\mathrm{HC}, \mathrm{CO}$ and $\mathrm{NO}$ to $\mathrm{NO}_{2}$. The application of the SCR enabled the engine to run with higher $\mathrm{NO}_{\mathrm{x}}$ levels for improved thermal efficiency. The increased $\mathrm{NO}_{2}$ level produced by the DOC facilitates the passive regeneration of the CPF, reducing the fuel consumption caused by periodic CPF active regeneration. For the heavy-duty diesel engine applications, the SCR was usually installed after the CPF. A decomposition tube with a DEF injector was installed between the CPF and the SCR. The injector supplies urea water solution into the exhaust. The $\mathrm{NH}_{3}$ produced from urea decomposition is the reductant reacting with $\mathrm{NO}_{\mathrm{x}}$ on the $\mathrm{SCR}$ catalytic surface. 


\subsection{Catalytic $\mathrm{NO}_{x}$ Reduction}

Catalytic $\mathrm{NO}_{x}$ reduction technologies have been used for emission control since the 1970's in industries for coal fired and diesel power plants [29,30]. The SCR catalyst formulations at that time included iron oxide, aluminum oxide, $\mathrm{TiO}_{2}$-based catalyst, and others. Among those formulations, the $\mathrm{TiO}_{2}$ based catalyst developed in 1973 had many advantages and were commercially implemented for stationary applications. At the same time, because of the growing concerns about $\mathrm{PM}$ and $\mathrm{NO}_{\mathrm{x}}$ emissions from diesel engines, efforts to implement catalytic $\mathrm{NO}_{x}$ reduction technology into diesel trucks were initiated in 1992 in Europe. $\mathrm{A} \mathrm{TiO}_{2}-\mathrm{V}_{2} \mathrm{O}_{5}$ type extruded SCR catalyst coupled with urea injection and metering system named SINOx [31] was installed into two heavy-duty diesel trucks by Siemens $A G$ and Siemens Westinghouse for on-road tests. The urea water solution with urea concentration of $32.5 \%$ by weight which provided lowest crystallization point was selected as the reducant. The $\mathrm{NO}_{\mathrm{x}}$ reduction efficiency achieved $60-80 \%$ during the 200,000 mile truck life and it proved that the SCR system in a diesel truck was able to meet Europe IV and $\mathrm{V} \mathrm{NO}_{\mathrm{x}}$ emission regulations [32]. In 1998, the Siemens-Westinghouse was requested to prepare the system for production by European truck manufactures including Daimler Chrysler and MAN [33].

Because of the success of the SINOx system in Europe, US companies started to demonstrate and evaluate the system in U.S. trucks in 1999 [33]. A representative study of the urea-SCR system with $\mathrm{TiO}_{2}-\mathrm{V}_{2} \mathrm{O}_{5}$ type catalyst was carried out in both an engine test cell and in a heavy-duty diesel truck [34]. The test results showed 56 and $71 \% \mathrm{NO}_{\mathrm{x}}$ reduction over the cold and hot US transient cycles respectively with low $\mathrm{NH}_{3}$ slip level. $A \mathrm{NO}_{x}$ reduction efficiency of $86 \%$ was achieved over a steady state cycle. $\mathrm{A} \mathrm{NO}_{\mathrm{x}}$ sensor calibrated to chemiluminescence $\mathrm{NO}_{\mathrm{x}}$ detector (CLD) was used for $\mathrm{NO}_{\mathrm{x}}$ measurement before and after the SCR system in road tests. The test results confirmed the average $\mathrm{NO}_{\mathrm{x}}$ reduction efficiency of $65 \%$ with the urea consumption rate of 94 miles per gallon (mpg). Other following studies also proved that the SCR system combined with DPF was able to meet the EPA 2007 regulations and potentially the 2010 regulations, but may need ultra-low sulfur diesel fuel and effective feedback $\mathrm{NO}_{x}$ control strategies $[35,36]$. 
The $\mathrm{NO}_{x}$ regulations for heavy-duty diesel engines in 2010 were further reduced by $80 \%$ compared to the EPA 2007 regulations. It required the application of the urea - SCR system along with the DOC/DPF. To make this happen, several different SCR formulations were developed to provide better $\mathrm{NO}_{x}$ reduction efficiency and catalyst durability. Extensive experimental efforts along with the modeling studies and control strategy development were carried out to further improve the system performance. Studies are also being carried out to reduce the aftertreatment system complexity and system cost by integrating SCR catalyst with the DPF. A study showed that a combined SCR on filter (SCRF) system with DOC installed upstream is able to provide similar $\mathrm{NO}_{x}$ reduction performance compared to a traditional channel flow Cu-zeolite SCR after extended hydrothermal aging equivalent to 120,000 mile catalyst life [37].

\subsection{SCR Formulations}

As mentioned in the previous section, the $\mathrm{TiO}_{2}$ based catalyst with active components of $\mathrm{V}_{2} \mathrm{O}_{5}$ or $\mathrm{WO}_{2}$ used in stationary applications was then applied to diesel engine $\mathrm{NO}_{x}$ reduction as the first generation of the SCR catalyst. It provided $90 \% \mathrm{NO}_{x}$ reduction between the range of 300 and $500^{\circ} \mathrm{C}$. The effective operating temperature of the $\mathrm{TiO}_{2^{-}}$ $\mathrm{V}_{2} \mathrm{O}_{5}$ catalyst is more active with the support of $\mathrm{WO}_{2}$. Its activity is usually limited to below $550^{\circ} \mathrm{C}$ because of the low melting point of $\mathrm{V}_{2} \mathrm{O}_{5}$ [38]. This limits its application in heavy-duty diesel engines because the SCR will be periodically exposed to temperature above $550^{\circ} \mathrm{C}$ when actively regenerating the DPF which is typically installed upstream of the SCR. The vanadia catalyst supported by titania is another formulation for stationary and diesel applications. It provides promising low temperature performance during cold start period with superior sulfur resistance and reasonable thermal durability [39,40]. A vanadia SCR catalyst was reported to be thermally stable under accelerated aging conditions which is equivalent to a 120,000 mile catalyst life [41]. A diesel aftertreatment system with vanadia based SCR placed upstream of the DOC/DPF showed higher than 95\% $\mathrm{NO}_{x}$ reduction over the Europe transient cycle (ETC) [42]. However, installing the SCR before the DOC/DPF exposes the catalyst directly to engine exhaust coming out of the turbocharger. The HC and PM contents in the exhaust affect the SCR performance 
by blocking the active catalyst sites and cause catalyst deactivation when oxidizing the $\mathrm{HC}$ and PM loaded on the catalyst at high temperatures.

The new generation of the SCR with metal based zeolite catalysts showed improved $\mathrm{NO}_{\mathrm{x}}$ reduction activities and thermal durability compared to the $\mathrm{TiO}_{2}$ and vanadia. As a result, iron (Fe) and copper $(\mathrm{Cu})$ zeolite SCR catalysts were selected for the heavy-duty diesel applications to meet the $\mathrm{NO}_{\mathrm{x}}$ emission regulations. The characteristics of the $\mathrm{Fe}-$ zeolite and $\mathrm{Cu}$ - zeolite SCR from different references [43-51] are compared and summarized as below.

- $\quad$ Cu-zeolite SCR provides better $\mathrm{NO}_{x}$ reduction efficiency than Fe-zeolite SCR at the temperatures below $350^{\circ} \mathrm{C}$ while Fe-zeolite SCR performs better at higher temperatures.

- Both catalysts have improved thermal durability in comparison to other catalytic formations, but extremely high temperature still can deactivate the catalysts. The not-to-exceed temperature is $775^{\circ} \mathrm{C}$ and $925^{\circ} \mathrm{C}$ respectively for Cu-zeolite and Fe-zeolite catalysts.

- Both catalysts show the ability to oxidize $\mathrm{NH}_{3}$ at temperatures above $300^{\circ} \mathrm{C}$, and Cu-zeolite is more active than Fe-zeolite for oxidizing $\mathrm{NH}_{3}$, resulting in less available $\mathrm{NH}_{3}$ for the $\mathrm{NO}_{x}$ reduction reactions. This also explains why $\mathrm{NO}_{x}$ reduction efficiency over Fe-zeolite is higher than that of Cu-zeolite at temperatures higher than $350^{\circ} \mathrm{C}$.

Cu-zeolite SCR has higher $\mathrm{NH}_{3}$ storage capacity than Fe-zeolite SCR. The $\mathrm{NO}_{\mathrm{x}}$ reduction efficiency of both catalysts is dependent on the amount of $\mathrm{NH}_{3}$ adsorbed on the catalyst surface. High temperature $\mathrm{NO}_{\mathrm{x}}$ reduction efficiency for both catalysts keeps increasing with increasing of $\mathrm{NH}_{3}$ stored on the catalyst. However, lower temperature $\mathrm{NO}_{x}$ reduction on Fe-zeolite SCR is inhibited by extra $\mathrm{NH}_{3}$ storage caused by active SCR sites being covered by $\mathrm{NH}_{3}$. But Cuzeolite SCR is not affected by its high $\mathrm{NH}_{3}$ storage capacity. 
- The $\mathrm{NO}_{x}$ reduction efficiency over Cu-zeolite SCR is less dependent on SCR inlet $\mathrm{NO}_{2} / \mathrm{NO}_{x}$ ratio compared to that of the Fe-zeolite. However, the Fe-zeolite SCR catalyst shows better $\mathrm{NO}_{x}$ reduction activity than the Cu-zeolite with an optimal of $\mathrm{NO}_{2} / \mathrm{NO}_{x}$ ratio of 0.5 .

- The $\mathrm{NO}+\mathrm{NH}_{3}$ reaction at lower temperatures is subjected to inhibition effects over the Fe-zeolite SCR, causing a reduction in the NO conversion efficiency shown in Figure 2.1. This effect was not observed over a Cu-zeolite SCR catalyst.

- The Fe-zeolite SCR catalyst is forms more $\mathrm{N}_{2} \mathrm{O}$ in the presence of excess $\mathrm{NO}_{2}$ compared to the Cu-zeolite SCR.

- $\quad$ For temperatures lower than $275^{\circ} \mathrm{C}$, both catalysts tend to form surface nitrates with $\mathrm{NO}_{2}$ and $\mathrm{NH}_{3}$ in the feed stream. The surface nitrates can be decomposed at high temperatures or reduced through reactions with NO. However, the cumulated surface nitrates on the catalytic surface are difficult to reduce when the NO fraction is low at temperatures below $275^{\circ} \mathrm{C}$, causing an inhibition effects on the $\mathrm{NO}_{\mathrm{x}}$ reduction efficiency.

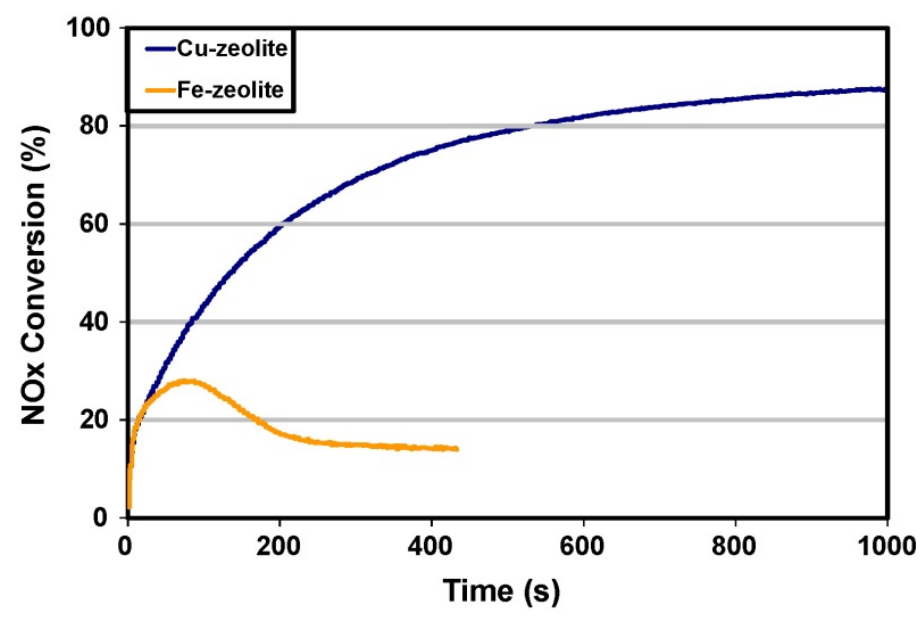

Figure 2.1: $\mathrm{NO}_{x}$ conversion performance at $200^{\circ} \mathrm{C}$ of Fe and Cu-zeolite SCR with 350 ppm of $\mathrm{NO}$ and $350 \mathrm{ppm}$ of $\mathrm{NH}_{3}$ in the gas flow [50] 
Both Fe and Cu-zeolite SCR catalysts have their advantages and disadvantages. Studies were carried out to combine both catalysts in order to obtain better performance compared to each individual formulation. The experimental results of a combined Fezeolite and Cu-zeolite catalyst was presented in reference [47]. In this study, a Fe-zeolite SCR was placed in front of a Cu-zeolite SCR. Different percentage combinations of the two catalysts were tested in a flow reactor. The results showed that the combined catalyst showed higher $\mathrm{NO}_{x}$ conversion efficiency at both high and low temperature which cannot be realized by a single catalyst formulation. It also concluded that a setup with $33 \%$ Fe-zeolite and $67 \%$ Cu-zeolite provided best steady-state $\mathrm{NO}_{\mathrm{x}}$ conversion performance. However, it was reported that the transient performance of the combined catalyst is not as good as the Cu-zeolite SCR at the temperatures below $300^{\circ} \mathrm{C}$ [47]. Another study was conducted to test three different combinations of the Fe-zeolite and Cu-zeolite catalysts. The 33\% Fe-zeolite $+67 \%$ Cu-zeolite was tested first and similar results were reported [52]. The second test was performed with the "mixed washcoat consisting equal fraction of $\mathrm{Fe}$ - and Cu-zeolites". The performance of this setup reached the average performance of the two catalysts [52]. The third setup tested was a dual layer system consisting of a layer of Fe-zeolite washcoat on top of a Cu-zeolite layer with the fraction of $33 \%$ and $67 \%$ for each formulation respectively. This combination achieved better performance than the other setups and each individual catalyst formulation [52].

\subsection{SCR Experimental Studies}

The SCR experimental studies include flow reactor tests, engine test cell tests, and on road tests. The representative experimental studies will be reviewed in this section.

\subsubsection{SCR Reactor Testing}

The flow reactor tests provide opportunities to collect SCR data under specific conditions that cannot be easily achieved in the engine testing environment. In a reactor setup, the temperature is typically controlled or maintained by an oven with temperature feedback control. Synthetic exhaust flow generated from calibration gas cylinders is used in 
reactor tests so that the inlet concentration of each species can be individually controlled. The test protocols can be designed for certain modeling or characterization purposes. The typical flow reactor hardware setups and reactor test design can be found in references $[43,46,50,53-58]$. The purposes of those reactor tests included collecting data for studying the SCR performance under different conditions and the SCR kinetics, mass transfer limitations, SCR poisoning by $\mathrm{HC}$ and sulfur contents, thermal aging and other factors affecting the SCR performance.

In reference [43], a flow reactor was setup to test the zeolite based SCR catalysts using a compact four step protocol. The basic components of the synthetic exhaust flow consisted of $10 \% \mathrm{O}_{2}, 8 \% \mathrm{CO}_{2}, 7 \% \mathrm{H}_{2} \mathrm{O}$ and balanced with $\mathrm{N}_{2}$. $\mathrm{NO}, \mathrm{NO}_{2}$ and $\mathrm{NH}_{3}$ were turned on and off at each different segment of the protocol to generate different $\mathrm{NO}_{2} / \mathrm{NO}_{\mathrm{x}}$ ratios and $\mathrm{NH}_{3} / \mathrm{NO}_{\mathrm{x}}$ ratios (ANR) at the SCR inlet. The flow rates of different gases were controlled by mass flow controllers. The test protocol used in the tests facilitated studies of oxidation reactions between $\mathrm{NO}$ and $\mathrm{NO}_{2}, \mathrm{NO}_{\mathrm{x}}$ storage on the catalyst, $\mathrm{NO}_{\mathrm{x}}$ conversion, $\mathrm{NH}_{3}$ slip and storage under SCR reaction conditions with different $\mathrm{NO}_{2} / \mathrm{NO}_{\mathrm{x}}$ ratios, $\mathrm{NH}_{3}$ oxidation and storage under $\mathrm{NH}_{3}$ saturation condition, and reactions between $\mathrm{NO}_{x}$ and storage $\mathrm{NH}_{3}$ after $\mathrm{NH}_{3}$ is turned off. The data collected from the reactor tests were used for SCR performance characterization and determining the kinetic parameters of the major SCR reactions [43].

Reactor studies to determine the influences of the $\mathrm{NO}_{2} / \mathrm{NO}_{\mathrm{x}}$ ratio and the ANR on Fezeolite and Cu-zeolite SCR performance were presented in references [56,59]. The test results showed that $\mathrm{Cu}$-zeolite $\mathrm{SCR}$ stored more $\mathrm{NH}_{3}$ and had better $\mathrm{NO}$ reduction activity but poor $\mathrm{NO}_{2}$ reduction performance under low temperatures because the formation of surface nitrates are more stable on Cu-zeolite SCR catalyst. The Fe-zeolite SCR gave better performance when the inlet $\mathrm{NO}_{2} / \mathrm{NO}_{x}$ ratio was higher than 0.5 . At temperatures from 350 to $400^{\circ} \mathrm{C}$, with the ANR slightly higher than one can significantly improve $\mathrm{NO}_{x}$ reduction efficiency without forming any $\mathrm{NH}_{3}$ slip. At low temperatures when ammonia storage capacity is high, reducing ANR to slightly lower than 1 can reduce $\mathrm{NH}_{3}$ slip level without significant impact on $\mathrm{NO}_{x}$ conversion efficiency. However, from a control viewpoint, it is still challenging to optimize the amount of $\mathrm{NH}_{3}$ stored on 
the SCR for different engine operating conditions to maximize $\mathrm{NO}_{\mathrm{x}}$ reduction efficiency and reduce $\mathrm{NH}_{3}$ slip, especially for transient engine operating conditions.

A new reactor test technique usually called "Spaci-MS" or Spaci-FTIR" (depending on which emission analyzer is used for emission measurements) has been developed and recently presented in the literature. The technique allows measuring the internal gaseous concentrations in catalyst monoliths [60-63]. The application of the Spaci-MS technique is limited compared to the Spaci-FTIR because of the difficulty in measuring low level of $\mathrm{NH}_{3}$ in the presence of $\mathrm{N}_{2}$ and $\mathrm{H}_{2} \mathrm{O}$ using the mass spectrometer. A typical setup schematic of the Spaci-FTIR test is shown in Figure 2.2. In reference [63], a gas phase MKS MultiGas 2030 FTIR was used for internal gaseous measurements of a Fezeolite SCR using the Spaci technique. A micro capillary that can be extended into different axial locations of catalyst monoliths was used to draw gas flow into the FTIR. Because the volume of the sampling gas from catalyst channels is limited compared to the gas cell of the FTIR, the sample gas was diluted with $\mathrm{N}_{2}$ under a specific ratio before feeding to the FTIR. The measured concentrations multiplied by the dilution ratio gave the gas concentrations at different axial locations. Compared to traditional reactor experiments, the spaci test results gave a better insight of the changes in gaseous concentrations along the catalyst channel and the relative reaction rate at different axial locations. With a carefully designed test protocol, the Spaci-FTIR technique also provides opportunities of determining the axial $\mathrm{NH}_{3}$ storage distribution, which is important to modeling and control strategy development efforts.

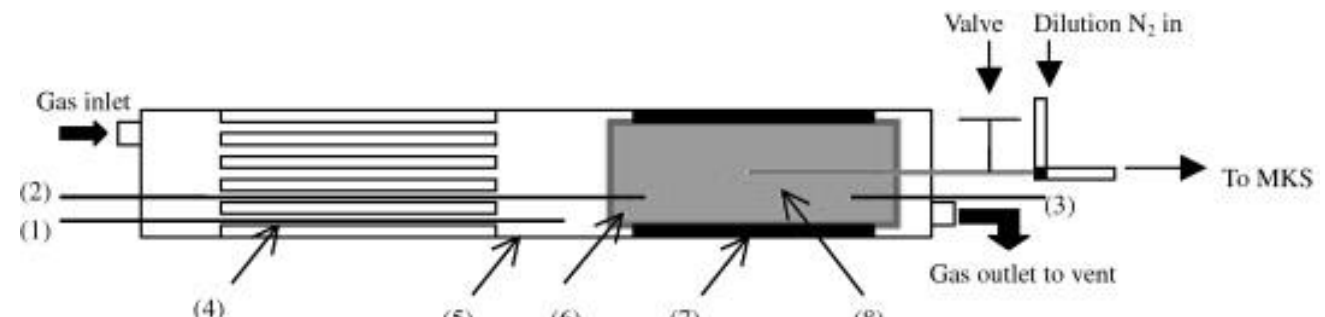

(4)

(5)

(6)

(7)

(8)

Figure 2.2: A schematic of the Spaci-FTIR test setup: “(1, 2, 3): thermocouples; (4) hollow quartz rods for gas preheating and distribution; (5) quartz tube reactor; (6) monolith Fe-zeolite catalyst; (7) insulation material; (8) fused silica capillary."[63] 
An alternative testing procedure was introduced in reference [64] to achieve the axial resolved measurements for a Cu-zeolite SCR by coating the core sample with a different length of catalytic washcoat. However, keeping the washcoat loading density consistent for different core samples tested was a problem.

The reactor testing technique provides opportunities for the SCR characterization and kinetics analysis. However, it simplifies the SCR exposure conditions in engine applications by introducing gaseous $\mathrm{NH}_{3}$ as reductant instated of urea water solution. The more complex engine exhaust conditions including the urea decomposition, flow, temperature and species maldistribution were not considered in the reactor tests.

\subsubsection{SCR Engine Testing}

The engine testing environment allows the SCR to be exposed to the real engine exhaust conditions. In an engine test cell, the engine is coupled with a dynamometer to control the engine speed and torque. Both steady-state and transient SCR tests can be performed in an engine test cell. Procedures for performing transient aftertreatment system tests in an engine dynamometer test cells can be found in reference [65].

A study of the performance of both Fe-zeolite and Cu-zeolite SCR catalysts were carried out in an engine test cell at Johnson Matthey Inc. [66]. The test cell consisted of a Model Year 2007 US medium duty diesel engine coupled with a 600 HP AC dynamometer. The aftertreatment system consisted of DOC, CPF, and SCR. A MKS FTIR and a Chemiluminescence $\mathrm{NO}_{x}$ detector were used for emission measurements. Ultra-low sulfur diesel and the $32.5 \%$ urea water solution were used in the tests. Steady state tests under different temperature and space velocity conditions with three different $\mathrm{NH}_{3} / \mathrm{NO}_{\mathrm{x}}$ ratios $(0.8,1.0$ and 1.3) and transient tests with a developed urea injection strategy were performed for both catalysts. The engine test results showed similar results with the reactor test results and confirmed the conclusion that Cu-zeolite provided better performance under low temperatures and better tolerance to the inlet 
$\mathrm{NO}_{2} / \mathrm{NO}_{\mathrm{x}}$ ratio. The paper also concluded that the Cu-zeolite SCR is less tolerant to the sulfur poisoning compared to the Fe-zeolite SCR.

Reference [67] presented a detailed engine aftertreatment system test procedure and a experimental data collection strategy to develop a "virtual diesel aftertreatment exhaust line" consisting of DOC, DPF, SCR, and ammonia oxidation catalyst (AMOX) models. The test cell consisted of a $13 \mathrm{~L} 355 \mathrm{~kW}$ diesel engine that met EPA 2007 regulations. The aftertreatment system setup consisted of a DOC, a DPF, two Fe-zeolite SCR bricks and an AMOX catalyst. A measurement setup for collecting the data used for model development and calibration was introduced in the paper. A FTIR was setup for emission measurements before and after each catalyst while a LDS Laser Diode analyzer was used for tailpipe $\mathrm{NH}_{3}$ measurements. The emissions at the SCR inlet was not measured using the analyzers because of the possible presence of incomplete urea decomposition products. Temperatures at different locations of the aftertreatment system as well as temperature gradients in the DOC and DPF substrates were measured. Pressure before and after each catalyst brick and the engine out PM were also measured. A measurement program specially designed for the purpose of calibrating the models was introduced to collect data under various conditions. The determined SCR test conditions included low, medium and high exhaust mass flow rates with post-DPF temperatures from 200 to $380^{\circ} \mathrm{C}$ as well as the DPF regeneration conditions. The performance of the calibrated virtual exhaust line was validated against transient test cycles. The possible applications of the virtual exhaust line were also introduced. However, a drawback of the test setup is that the gaseous concentrations after a catalyst brick might not be uniformly distributed. This would cause incorrect emission measurements when sampling right after the brick, especially after the SCR when the urea injected upstream is not well mixed with the exhaust flow.

On road vehicle tests were conducted to test the system reliability, durability and field performance under real driving conditions [36,68,69]. Portable emission analyzers or sensors are required for emission measurements during on road vehicle testing. The vehicle aged catalyst after significant driving hours can be used for laboratory testing to study the aging effects on the SCR performance. The on road tests did not contribute to 
studying the characteristics of the SCR or developing the SCR model, therefore it will not be discussed in further detail.

\subsection{SCR Modeling Studies}

A SCR model usually consists of chemical reaction mechanisms and their associated kinetics, mass balances of gaseous species, and an energy balance. This section will be focusing on the literature that are related to the SCR reaction mechanisms, modeling of the mass transfer and heat transfer for the SCR system.

\subsubsection{SCR Reaction Mechanisms}

As discussed in previous sections, a calibrated high fidelity SCR model is important for system characterization, optimization, control strategy and OBD development. Selection of the SCR reaction mechanisms is important for a SCR model to simulate the reaction system. The experimental and modeling findings and accomplishments about the SCR reaction mechanisms from the literature are presented. The global chemical reactions for the urea SCR system include urea decomposition reactions that produce $\mathrm{NH}_{3}$ and the SCR reactions that take place on the catalytic surface.

For the urea-SCR, the reductant is introduced by injecting the urea-water solution into the exhaust stream. A numerical model simulating the interaction of the spray and the surrounding exhaust gas is presented in reference [70]. The injected urea water solution then goes through a complex process to produce $\mathrm{NH}_{3} . \mathrm{NH}_{3}$ is released through hydrolysis and thermal decomposition of the aqueous urea. After the urea-water solution is injected, evaporation of the water from small droplets formed by high pressure injection leads to formation of solid or molten urea as shown in Equation 2.1, followed by a urea decomposition process which can be divided into two steps [71-74]. The first step is the hydrolysis process of the molten urea producing ammonia and isocyanic acid are given in Reaction 2.2. Then in the second step, hydrolysis of isocyanic acid takes place and produces ammonia and $\mathrm{CO}_{2}$ as given in Reaction 2.3. Isocyanic acid is stable in the gas phase and requires a catalytic surface to accelerate the hydrolysis reaction [75]. 


$$
\begin{gathered}
\left(\mathrm{NH}_{2}\right)_{2} \mathrm{CO} \text { (aqueous) } \rightarrow\left(\mathrm{NH}_{2}\right)_{2} \mathrm{CO} \text { (molten) }+\mathrm{x} \mathrm{H}_{2} \mathrm{O} \\
\left(\mathrm{NH}_{2}\right)_{2} \mathrm{CO} \text { (molten) } \rightarrow \mathrm{NH}_{3}+\mathrm{HNCO} \\
\mathrm{HNCO}+\mathrm{H}_{2} \mathrm{O} \rightarrow \mathrm{NH}_{3}+\mathrm{CO}_{2}
\end{gathered}
$$

The modeling studies to simulate the urea injection and decomposition process can be found in the literature $[70,76,77]$. However, because of the complexity of this process, it was not included in most of the SCR models. It was assumed that gas phase $\mathrm{NH}_{3}$ instead of urea and its incomplete decomposition products was present at the SCR inlet.

The ammonia formation process may occur all the way from injection of the urea-water solution until the mixed flow passes the first section of the SCR catalyst, and its completion is dependent on the exhaust temperature. Each of the reactions given in 2.1, 2.2, and 2.3 starts at the temperature of 193,250 , and $400^{\circ} \mathrm{C}$ respectively [71]. At exhaust temperatures below $300 \mathrm{C}$, less than $20 \%$ of urea decomposition is complete before going into the SCR and the rest of urea decomposition process takes place on the SCR catalyst surface [73]. This is considered to be one reason that the SCR performance in the engine environment is lower than in the reactor tests [77]. Additionally, the process shown in Equation 2.1 cannot be complete under situations of low exhaust temperature and poor mixing, leading to urea deposits or urea crystallization formation on the exhaust pipes.

The urea decomposition process is complex and has several pathways forming side products including $(\mathrm{CNOH})_{3}$ (cyanuric acid) and $\mathrm{C}_{3} \mathrm{H}_{6} \mathrm{~N}_{6}$ (melamine), as reported in reference [78]. The pathway reactions are not significant and no by-products have been observed post SCR, but the byproducts can form solid deposits in the exhaust pipe or on the catalyst surface. These factors can significantly affect the SCR performance when applied to engine exhaust. For vehicle applications, urea injection is not initiated at low exhaust temperature conditions in order to prevent large amounts of urea formatting deposits. 
A laboratory and engine study of the urea deposit formation and vaporization on Fezeolite and Cu-zeolite SCR catalysts is presented in reference [72]. A core SCR sample was tested with laboratory urea dripping onto the catalyst in a flow reactor at a space velocity of $15,000 \mathrm{hr}^{-1}$ and temperatures from 150 to $350 \mathrm{C}$. In the engine tests, urea decomposition products and solid deposits were analyzed. A powder X-ray diffraction (XRD) analyzer and a Mattson Instruments Cygnus 100 FTIR were used for analyzing the diffraction patterns and the infrared spectra of the solid deposits collected from the reactor and engine tests. An MKS FTIR was used to measure the urea decomposition products in the engine tests. The results showed that the deposits formed in reactor tests were similar to the samples collected from engine tests. The urea- related deposits were formed below a temperature of $300^{\circ} \mathrm{C}$ and large fraction of the deposits (more than $95 \%$ ) can be vaporized at temperatures higher than $350^{\circ} \mathrm{C}$. The solid deposits formed contained a series of components including urea and urea decomposition byproducts (biuret, cyanuric acid, etc.). Based on the analysis of a thermal gravimetric test, it was determined that different components vaporized at different temperatures.

The SCR reaction mechanisms are complex and include a series of pathway reactions. However, several global reactions were selected in the literature to represent the complex reaction mechanisms. The global $\mathrm{NO}_{\mathrm{x}}$ reduction reactions take place between the species in the surface phase (an assumed thin layer on the catalytic surface) and the $\mathrm{NH}_{3}$ stored on the catalyst. The $\mathrm{NH}_{3}$ adsorption/desorption reaction shown in Reaction 2.4 is considered as the first step of the SCR reaction mechanisms. The stored $\mathrm{NH}_{3}$ then reacts with other species in the surface phase. $S^{*}$ refers to the storage sites on the catalyst and $\mathrm{NH}_{3}{ }^{*}$ refers to the stored $\mathrm{NH}_{3}$. Most of the SCR models published have only one type of $\mathrm{NH}_{3}$ storage site which stores $\mathrm{NH}_{3}$ and supports all SCR reactions. However, Choi proved that there were two types of reaction sites on a SCR catalytic surface by performing temperature-programmed desorption (TPD) tests over the SCR catalyst [79]. Reference [23] also showed that adding a second reaction site which only supports $\mathrm{NH}_{3}$ adsorption and desorption to the Fe-zeolite SCR model improved the model performance. A second reaction site improves the $\mathrm{NH}_{3}$ adsorption and desorption performance of the model and adds more kinetic parameters to be identified. 


$$
\mathrm{NH}_{3}+\mathrm{S}^{*} \leftrightarrow \mathrm{NH}_{3}^{*}
$$

As described in the literature [80-86], there are three dominant global reactions between the stored $\mathrm{NH}_{3}, \mathrm{NO}$ and $\mathrm{NO}_{2}$ taking place on the SCR surface for different SCR formulations including $\mathrm{TiO}_{2}$-based, titania-based, Cu-zeolite, and Fe-zeolite SCR. The first global reaction introduced is called the standard SCR reaction which takes equal molar of $\mathrm{NH}_{3}$ and $\mathrm{NO}$ to react in the presence of $\mathrm{O}_{2}$. This chemical reaction is shown in Reaction 2.5 [82]. It was reported in reference [50] that the catalyst exhibited two separate functions on two types of catalyst sites under standard SCR condition. The catalyst support behaved as an acid site which adsorbed $\mathrm{NH}_{3}$ while the transition metal oxidized $\mathrm{NO}$ and formed "surface $\mathrm{NO}_{x}$ adsorption complexes" which react with $\mathrm{NH}_{3}$ afterwards [50]. The excess $\mathrm{NH}_{3}$ in the gas flow has more effect on the NO oxidation process for the Fe-zeolite SCR than the Cu-zeolite SCR and it explains the $\mathrm{NH}_{3}$ inhibition effects observed over Fe-zeolite SCR. However, this mechanism and explanation is still debatable and the detailed mechanism of the standard SCR reaction is still under investigation.

$$
4 \mathrm{NH}_{3}+4 \mathrm{NO}+\mathrm{O}_{2} \rightarrow 4 \mathrm{~N}_{2}+6 \mathrm{H}_{2} \mathrm{O}
$$

This reaction plays an important role for $\mathrm{NO}_{x}$ reduction if the SCR is placed in front of the DOC and the CPF because about $90 \%$ of diesel engine out $\mathrm{NO}_{x}$ is $\mathrm{NO}$. For applications where the SCR is located after a DOC and CPF, the $\mathrm{NO}_{x}$ composition will be different because of the $\mathrm{NO}$ and $\mathrm{NO}_{2}$ involved reactions across these first two components. In the situation with the SCR inlet molecular $\mathrm{NO} / \mathrm{NO}_{2}$ ratio of 1 , the second global SCR reaction which is given in Reaction 2.6 will become dominant. It is called fast SCR reaction because it has the highest reaction rate among all global SCR reactions reported. As a result, controlling the SCR inlet $\mathrm{NO} / \mathrm{NO}_{2}$ ratio close to unity will promote the fast SCR reaction and improve $\mathrm{NO}_{\mathrm{x}}$ reduction efficiency [87].

$$
2 \mathrm{NO}+2 \mathrm{NO}_{2}+4 \mathrm{NH}_{3} \rightarrow 4 \mathrm{~N}_{2}+6 \mathrm{H}_{2} \mathrm{O}
$$

The fast SCR reaction was reported to be a combination of a series of step reactions over a Fe-zeolite SCR catalyst [49]. This reaction mechanism was separated into two steps which were the formation of ammonia nitrates and consumption of ammonia 
nitrates. The reactions included in the first step are given in Reactions 2.7-2.10, and the reactions included in the second step are given in Reactions 2.11-2.13. It can be seen that there are several intermediate products that can be consumed right away. Those intermediate products include nitrous acid ( $\mathrm{HONO})$, nitric acid $\left(\mathrm{HNO}_{3}\right)$ and ammonium nitrate $\left(\mathrm{NH}_{4} \mathrm{NO}_{3}\right)$.

$\begin{array}{cr}2 \mathrm{NO}_{2} \leftrightarrow \mathrm{N}_{2} \mathrm{O}_{4} & 2.7 \\ \mathrm{~N}_{2} \mathrm{O}_{4}+\mathrm{H}_{2} \mathrm{O} \leftrightarrow \mathrm{HONO}+\mathrm{HNO}_{3} & 2.8 \\ \mathrm{NH}_{3}+\mathrm{HONO} \leftrightarrow \mathrm{N}_{2}+2 \mathrm{H}_{2} \mathrm{O} & 2.9 \\ \mathrm{NH}_{3}+\mathrm{HNO}_{3} \leftrightarrow \mathrm{NH}_{4} \mathrm{NO}_{3} & 2.10 \\ \mathrm{NH}_{4} \mathrm{NO}_{3} \leftrightarrow \mathrm{NH}_{3}+\mathrm{HNO}_{3} & 2.11 \\ \mathrm{HNO}_{3}+\mathrm{NO} \leftrightarrow \mathrm{NO}_{2}+\mathrm{HONO} & 2.12 \\ \mathrm{NH}_{3}+\mathrm{HONO} \rightarrow \mathrm{N}_{2}+2 \mathrm{H}_{2} \mathrm{O} . & 2.13\end{array}$

A study of a copper-on-alumina SCR catalyst reported the surface nitrates formation impacts the $\mathrm{NH}_{3}$ oxidation in presence of $\mathrm{NO}$ [88]. Reference [89] reported a "NO selfinhibition effect" below the temperature of $300^{\circ} \mathrm{C}$ over a Cu-zeolite SCR catalyst. It also concluded that the effect was caused by the $\mathrm{NO}_{x}$ and ammonium nitrate formed from $\mathrm{NO}_{x}$ and $\mathrm{NH}_{3}$ under low temperatures occupying and blocking the active catalytic site. Reference [48] reported a similar phenomenon which was the fast SCR reaction being blocked by $\mathrm{NH}_{3}$ over a Fe-zeolite SCR catalyst. All these phenomena can be explained by the mechanisms introduced above. When $\mathrm{NH}_{3}$ is present in the exhaust flow, the $\mathrm{HNO}_{3}$ formed when $\mathrm{NO}_{2}$ and $\mathrm{H}_{2} \mathrm{O}$ react with $\mathrm{NH}_{3}$ to form $\mathrm{NH}_{4} \mathrm{NO}_{3}$ (Reaction 2.10) which is stable at low temperatures. The reaction rate of Reaction 2.11 which releases $\mathrm{HNO}_{3}$ is limited by the low temperature, causing no $\mathrm{HNO}_{3}$ to react with $\mathrm{NO}$. So the reason of the inhibition was not actually because of the $\mathrm{NH}_{3}$ blocking the fast SCR reaction, but the $\mathrm{NH}_{4} \mathrm{NO}_{3}$ formation interrupted the following step reactions. Reducing $\mathrm{NH}_{3}$ concentration at this point would help move the step reactions forward to increase NO reduction efficiency. As a result, from the application point of view, high $\mathrm{NO}_{2}$ accompanied with high $\mathrm{NH}_{3}$ concentrations at the SCR inlet should be avoided at low temperatures because of the surface ammonium nitrate formation inhibiting the $\mathrm{NO}_{\mathrm{x}}$ reduction performance as discussed above. 
A chemical model of an integrated SCR-on-DPF system was introduced in Reference [90]. The model included the ammonium nitrate formation from $\mathrm{NO}_{2}$ and $\mathrm{NH}_{3}$ which is a reversible reaction as shown in Reaction 2.14. The ammonium nitrate formed can adsorb and desorb from the catalyst storage site. A similar mechanism for the nitrates formation was also included in a kinetic model for a $\mathrm{V}_{2} \mathrm{O}_{5}-\mathrm{WO}_{3} / \mathrm{TiO}_{2}$ urea-SCR catalyst [91].

$$
2 \mathrm{NH}_{3}+2 \mathrm{NO}_{2}+\mathrm{S}^{*} \leftrightarrow \mathrm{NH}_{4} \mathrm{NO}_{3}{ }^{*}+\mathrm{N}_{2}+\mathrm{H}_{2} \mathrm{O}
$$

The reaction between $\mathrm{NO}_{2}$ and $\mathrm{NH}_{3}$ on a SCR catalyst is slower than the reactions described in Reactions 2.5 and 2.6. It is called the slow SCR reaction and is shown in Reaction 2.15 .

$$
4 \mathrm{NH}_{3}+3 \mathrm{NO}_{2} \rightarrow 7 / 2 \mathrm{~N}_{2}+6 \mathrm{H}_{2} \mathrm{O}
$$

In addition to the slow SCR reaction, there are pathway reactions generating $\mathrm{N}_{2} \mathrm{O}$ in the $\mathrm{NH}_{3}-\mathrm{NO}_{2}$ reaction mechanisms [73] with the pathway reactions given in Reaction 2.16 and 2.17. The amount of $\mathrm{N}_{2} \mathrm{O}$ formed from $\mathrm{NH}_{3}-\mathrm{NO}_{2}$ reaction mechanisms over $\mathrm{Fe}-$ zeolite SCR is temperature dependent. $\mathrm{N}_{2} \mathrm{O}$ generated is greatest between the temperature range of 300 and $350^{\circ} \mathrm{C}$, then drops off at both lower and higher temperatures [23]. The Cu-zeolite SCR tends to form less $\mathrm{N}_{2} \mathrm{O}$ than the Fe-zeolite.

$$
\begin{gathered}
6 \mathrm{NH}_{3}+8 \mathrm{NO}_{2} \rightarrow 7 \mathrm{~N}_{2} \mathrm{O}+9 \mathrm{H}_{2} \mathrm{O} \\
4 \mathrm{NH}_{3}+4 \mathrm{NO}_{2}+\mathrm{O}_{2} \rightarrow 4 \mathrm{~N}_{2} \mathrm{O}+6 \mathrm{H}_{2} \mathrm{O}
\end{gathered}
$$

Another $\mathrm{N}_{2} \mathrm{O}$ formation reaction can be expressed as the global reaction

$$
2 \mathrm{NH}_{3}+2 \mathrm{NO}_{2} \rightarrow \mathrm{N}_{2} \mathrm{O}+\mathrm{N}_{2}+3 \mathrm{H}_{2} \mathrm{O} \text {. }
$$

$\mathrm{N}_{2} \mathrm{O}$ formation is also observed even if there is no $\mathrm{NO}_{2}$ in the SCR inlet stream for a Cuzeolite SCR at temperatures higher than $250^{\circ} \mathrm{C}$ with peak formation rate between 300 and $350^{\circ} \mathrm{C}$ [87]. However, this $\mathrm{NO}_{2}$ formed is not stable in the presence of $\mathrm{NH}_{3}$, and it is 
assumed that $\mathrm{N}_{2} \mathrm{O}$ is consumed as fast as it is produced under certain situations, so $\mathrm{N}_{2} \mathrm{O}$ exists for only a short period of time [23]. This $\mathrm{N}_{2} \mathrm{O}$ consumption reaction is given as

$$
3 \mathrm{~N}_{2} \mathrm{O}+2 \mathrm{NH}_{3} \rightarrow 4 \mathrm{~N}_{2}+3 \mathrm{H}_{2} \mathrm{O}
$$

The ammonia oxidation reaction needs to also be taken into consideration for SCR chemical mechanisms, especially for temperatures higher than $300^{\circ} \mathrm{C}$ [92]. The ammonia oxidation reaction proceeds selectively to $\mathrm{N}_{2}$ (given in Reaction 2.20)instead of NO (given in Reaction 2.21) [51,93]. The selectivity to $N_{2}$ is greater than $90 \%$ at the temperatures below $600^{\circ} \mathrm{C}$, and the selectivity to $\mathrm{NO}$ may only start at temperatures higher than $500^{\circ} \mathrm{C}$ for the Fe-zeolite catalyst [93]. It was reported that byproducts including $\mathrm{NO}, \mathrm{NO}_{2}$, and $\mathrm{N}_{2} \mathrm{O}$ were detected in $\mathrm{NH}_{3}$ oxidation products at temperatures above $400^{\circ} \mathrm{C}$ for a Cu-zeolite SCR as shown in Figure 2.3 [46], which is evidence that $\mathrm{NH}_{3}$ is oxidized to $\mathrm{NO}_{x}$. However, no byproduct was found below $400^{\circ} \mathrm{C}$ [37]. The activation energy of Reaction 2.20 is estimated to be about $20 \mathrm{kcal} / \mathrm{gmol}$ over a Fezeolite catalyst, and $24 \mathrm{kcal} / \mathrm{gmol}$ over a vanadium-based catalyst [51].

$$
\begin{gathered}
4 \mathrm{NH}_{3}+3 \mathrm{O}_{2} \rightarrow 2 \mathrm{~N}_{2}+3 \mathrm{H}_{2} \mathrm{O} \\
\mathrm{NH}_{3}+5 / 4 \mathrm{O}_{2} \rightarrow \mathrm{NO}+3 / 2 \mathrm{H}_{2} \mathrm{O}
\end{gathered}
$$

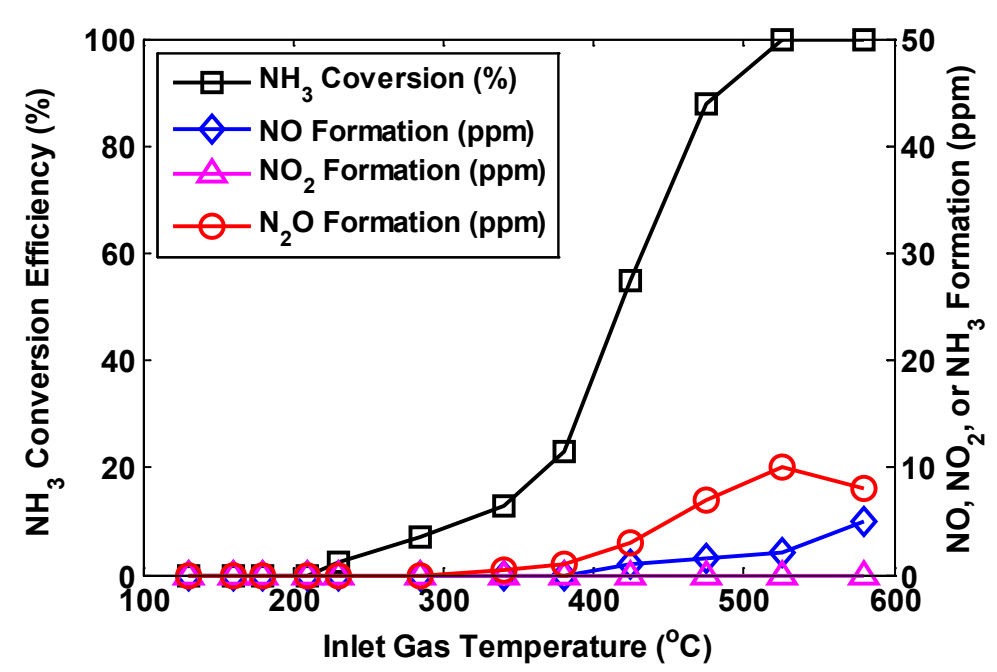

Figure 2.3: $\mathrm{NH}_{3}$ oxidation in the absence of $\mathrm{NO}_{x}$ as a function of temperature over a Cubased SCR under the space velocity of $30,000 \mathrm{hr}^{-1}$ [46] 
It is described in references $[43,94]$ that both the $\mathrm{NO}_{2}$ decomposition reaction and the $\mathrm{NO}$ oxidation reaction are taking place on the SCR catalytic surface. $\mathrm{NO}_{2}$ is decomposed to $\mathrm{NO}$ at temperatures higher than $450^{\circ} \mathrm{C}$ on a Cu-zeolite catalyst, and this $\mathrm{NO}_{2}$ reduction reaction is reversible, which means that $\mathrm{NO}$ can also be oxidized to $\mathrm{NO}_{2}$. $\mathrm{NO}$ oxidation to $\mathrm{NO}_{2}$ never exceeds $10 \%$ at any temperatures [94]. The activation energy of the $\mathrm{NO}_{2}$ reduction reaction was reported to be substantially higher than the $\mathrm{NO}$ oxidation reaction [43]. This reversible reaction is given as

$$
2 \mathrm{NO}+\mathrm{O}_{2} \leftrightarrow 2 \mathrm{NO}_{2}
$$

In reference [23], it was presented that overconsumption of $\mathrm{NH}_{3}$ under standard SCR reaction conditions was determined for a Fe-zeolite SCR catalyst based on reactor experimental data. It consumed more $\mathrm{NH}_{3}$ to reduce a certain amount of $\mathrm{NO}$ and the $\mathrm{NH}_{3}$ oxidation reactions were not able to explain the missing $\mathrm{NH}_{3}$. A second standard SCR reaction shown in Reaction 2.23 with $\mathrm{NH}_{3} / \mathrm{NO}$ ratio of $5 / 3$ in the reactants was introduced. The $2^{\text {nd }}$ standard SCR reaction is the combination of the standard SCR reaction (2.5) and the reaction of $\mathrm{NH}_{3}$ oxidation to $\mathrm{NO}$ (2.21). At low temperatures when $\mathrm{NH}_{3}$ oxidation to $\mathrm{NO}$ did not take place, $\mathrm{NH}_{3}$ overconsumption under $\mathrm{NO}+\mathrm{NH}_{3}$ reaction condition was still observed, hence it is reasonable to add the $2^{\text {nd }}$ reaction.

$$
5 \mathrm{NH}_{3}+3 \mathrm{NO}+9 / 4 \mathrm{O}_{2} \rightarrow 4 \mathrm{~N}_{2}+15 / 2 \mathrm{H}_{2} \mathrm{O}
$$

A Cu-zeolite SCR model was introduced in reference [94]. A 1-D numerical kinetic model for Cu-zeolite SCR was developed and calibrated based on reactor experimental data. The model is capable of simulating the SCR reactions and predicting SCR outlet NO, $\mathrm{NO}_{2}, \mathrm{NH}_{3}$ and $\mathrm{N}_{2} \mathrm{O}$ concentrations. $\mathrm{H}_{2} \mathrm{O}$ storage and desorption was included in the model to improve the temperature prediction performance during the cold start period. The model had a single storage site that supported $\mathrm{NH}_{3}$ adsorption, desorption and other SCR reactions including standard, fast, slow SCR reactions, $\mathrm{N}_{2} \mathrm{O}$ formation, two $\mathrm{NH}_{3}$ oxidation reactions, $\mathrm{NO}$ oxidation reaction which is a reversible reaction of the $\mathrm{NO}_{2}$ decomposition reaction, and an unusual reaction shown in Reaction 2.24 which can explain the phenomenon that the $\mathrm{NO}_{2}$ to $\mathrm{NO}$ conversion in the presence of $\mathrm{NH}_{3}$ was greater than the $\mathrm{NO}_{2}$ decomposition reaction in absence of $\mathrm{NH}_{3}$. The model was 
calibrated to reactor data and the model performance was validated to transient engine data. It was found more challenging for the model to predict the engine data because a series of factors including errors in the model inlet $\mathrm{NH}_{3}$ concentrations, simple rate equations for the reaction mechanisms, incomplete urea decomposition and $\mathrm{NH}_{4} \mathrm{NO}_{3}$ deposition in engine tests.

$$
2 \mathrm{NH}_{3}+3 \mathrm{NO}_{2} \rightarrow 3 \mathrm{NO}+\mathrm{N}_{2}+3 \mathrm{H}_{2} \mathrm{O}
$$

Although the SCR reaction mechanisms have been studied and presented in the literature, it is not able to cover all the step reactions and pathways that are taking place in the SCR. It is also too complex for a model to include all the SCR reactions. Careful selection of the global chemical reactions mechanisms is important in developing a model that effectively simulates the SCR reaction system.

\subsubsection{Mass Transfer}

A simplified one-dimensional SCR model which considers one single SCR channel is used by many researchers in order to reduce model computation time. SCR models over different catalytic formulations including Vanadia-titania SCR [95,96], Fe-zeolite SCR [97,98], Cu-zeolite SCR [94] have been published. For the SCR models published, EleyRideal mechanism which assumes only one of the reactants gets adsorbed on the catalytic surface and reacts with other reactants in the surface phase is commonly used to model the reaction mechanisms over the SCR catalyst. It's assumed that $\mathrm{NH}_{3}$ is the only species adsorbed on the catalyst, and the adsorbed $\mathrm{NH}_{3}$ reacts with other species in the surface phase. Gaseous species in the surface phase are assumed to be transported from the gas phase which refers to the bulk flow through the catalyst channel.

The mass transfer process is a factor affecting the SCR performance. Modeling the mass transfer between gases in gas and surface phases is important for developing the SCR model and improving the model accuracy in the mass transfer controlled regime [99]. 
The importance of modeling the mass transfer of channel flow catalysts have been addressed and studied [100-102]. Many studies on correlations of the Sherwood number which refers to a dimensionless mass transfer coefficient have been reported in the literature. The Sherwood number $(S h)$ correlations (a represent of the ratio between convective and diffusive mass transport) for difference channel shapes can be found in references [100,101]. A correlation for the Sherwood number for automobile catalyst converter applications was developed based on monolith reactor tests and was introduced in Reference [103]. The correlation of the Sherwood number is given in Equation 2.25.

$$
S h=0.766 \cdot\left(\frac{d}{L} \cdot \operatorname{Re} \cdot S c\right)^{0.483}, \quad\left(0.8<\frac{d}{L} \cdot \operatorname{Re} \cdot S c<130\right)
$$

Where, $d$ and $L$ are the diameter and the length of the channel. $R e$ is the Reynolds number which is the ratio of inertial force to viscous force. Sc is the Schmidt number which represents the ratio of the momentum diffusivity and mass diffusivity. The calculations of $R e$ and $S c$ are given in Equations 2.26 and 2.27 respectively.

$$
\begin{gathered}
\mathrm{Re}=\frac{\rho Q D_{H}}{\mu A} \\
S c=\frac{\mu}{\rho D}
\end{gathered}
$$

Where, $\rho$ is the density of the exhaust flow, $Q$ is the standard volumetric flow rate, $D_{H}$ is the hydraulic diameter of the catalyst channel, $\mu$ is the dynamic viscosity of the exhaust flow in, $A$ is the cross sectional area of the channel, $D$ is the mass diffusivity.

A comparison of the Sherwood number correlation shown in Equation 2.25 above with several other correlations in the literature given in Equations 2.28, 2.29 and 2.30 was presented in reference [104].

$$
S h=2.976 \cdot\left(1+0.095 \cdot \frac{d}{L} \cdot \operatorname{Re} \cdot S c\right)^{0.45}
$$




$$
\begin{gathered}
S h=0.705 \cdot\left(\frac{d}{L} \cdot \operatorname{Re}\right)^{0.45} \cdot S c^{0.56} \\
S h=2.709 \cdot\left(\frac{L}{z} \cdot \operatorname{Re} \cdot S c\right)^{0.45}
\end{gathered}
$$

Where, $z$ is the axial distance from the inlet the channel. And $L$ is the length of the catalyst channel. The results showed that each correlation showed a different Sherwood number distribution along the channel length because of the differences in catalyst specifications. Different Sherwood number correlations can affect the SCR model performance.

The mass transfer effects on the SCR model performance was studied in reference $[104,105]$. It was concluded that the higher Sherwood number resulted in better $\mathrm{NO}_{\mathrm{x}}$ conversion performance and the effects increased along with increase in the temperature and space velocity. The mass transfer effect on the SCR reactions is a function of the temperature and $\mathrm{NO}_{2} / \mathrm{NO}_{\mathrm{x}}$ ratio. At low temperatures when there is $\mathrm{NO}_{2}$ present, the change in Sherwood number on the $\mathrm{NO}_{\mathrm{x}}$ conversion becomes more obvious [104].

The mass transfer process discussed above is classified as external mass transfer, which refers to the diffusion process of the species in bulk flow to the catalytic surface. Other mass transfer processes in a SCR catalyst include: (1) the diffusion of species in the pores of the catalyst washcoat which referred as internal mass transfer (2) the diffusion of species within the nano pores of the zeolite crystallites (3) the convective flow through the channels of the monolithic catalyst $[99,106]$. The effects of both external and internal mass transfer on different SCR reactions over Fe-and Cu-zeolite SCR catalysts were studied through reactor tests with different catalyst dimensions and washcoat loadings [99]. The washcoat diffusion limitations on different reactions were determined: (1) the $\mathrm{NH}_{3}$ oxidation was affected by washcoat diffusion for both Fe-and Cu-zeolite SCR while the effects under low temperatures over the Cu-zeolite SCR was more pronounced (2) the diffusion limitations for the standard SCR reaction were important for both formulations in different temperature ranges (3) the washcoat diffusion limitations were observed for the fast and slow SCR reactions over both catalyst 
formulations in the temperature range of 300 to $500 \mathrm{C}(4)$ the NO oxidation reaction was not affected by the washcoat diffusion [99].

\subsubsection{Heat and Energy Transfer}

Modeling of the heat and energy transfer for the heavy-duty diesel aftertreatment system is usually applied to the DOC and CPF since there are significant temperature gradients when oxidizing the $\mathrm{HC}$ and PM. The SCR temperatures are more constant from inlet to the out under steady state conditions because of the small enthalpy change for the SCR reactions. A temperature difference of less than $4^{\circ} \mathrm{C}$ between the inlet and outlet of the catalyst was observed from the Fe-zeolite SCR experimental data [23]. Isothermal assumption was made for most of the published SCR models. When operating under transient engine conditions, the temperature gradients in the SCR catalyst cannot be neglected because of the heat transfer between the exhaust flow, the substrate and the ambient. Including the heat transfer in the SCR model is important for improving the simulation results under transient conditions.

The modeling of heat transfer for a DOC catalyst was introduced in the literature $[107,108]$. The heat transfer between exhaust flow and the substrate and between the substrate and the ambient were considered. Two energy balance equations respectively for the gas phase and the substrate were used to model the heat transfer.

\subsection{SCR Deactivation Effects}

Although the durability of the Fe- and Cu- SCR catalysts has been proved as discussed in previous sections, the activity of the catalysts may become deactivated after being exposed to $\mathrm{HC}$ or sulfur compounds and long terms of hydrothermal conditions. Other factors including the uniformity of the exhaust flow and the gaseous concentrations going into the SCR also affects the overall SCR performance in terms of $\mathrm{NO}_{x}$ conversion efficiency and $\mathrm{NH}_{3}$ slip. The $\mathrm{HC}$ poisoning, thermal aging, as well as the maldistribution effects on the SCR will be discussed in this section. Ultra-low sulfur diesel with sulfur 
concentration of less than $15 \mathrm{ppm}$ is used in the US since 2006, therefore, the sulfur poisoning effect is negligible [46].

\subsubsection{SCR HC Poisoning Effect}

Diesel fuel is a combination of several thousand hydrocarbons including saturated hydrocarbons and aromatic hydrocarbons, .etc. The unburned hydrocarbons in diesel exhaust compete with ammonia for occupying active SCR catalytic sites below temperatures of $300^{\circ} \mathrm{C}$ [109], as a result, blocking the active sites and reducing $\mathrm{NO}_{\mathrm{x}}$ reduction performance of the SCR $[110,111]$. For heavy-duty diesel engine applications, the SCR is typically used in a combination with a DOC and CPF in front of the SCR, which under normal conditions oxidize most of hydrocarbons in the diesel exhaust. In the case of a DOC and/or CPF failure, the SCR will be exposed to higher concentrations of hydrocarbons. Alternatively, in applications such as light-duty vehicles, the SCR is located right after the turbocharger and the hydrocarbons go directly into the SCR in this situation. As a result, it is important to understand the effect of hydrocarbons on SCR performance.

A study of the effect of hydrocarbons on a low temperature SCR was carried out on a bench reactor system [111]. Decane, ethylene and benzene were used as surrogate hydrocarbons representing the main components of diesel hydrocarbons. It was observed that $\mathrm{NO}_{\mathrm{x}}$ reduction over the SCR was inhibited with injection of the surrogate hydrocarbons. Benzene showed the most negative effect on $\mathrm{NO}_{\mathrm{x}}$ reduction activity. $\mathrm{A}$ series of tests were also carried out to determine suitable conditions for regenerating a SCR poisoned by hydrocarbons [111].

A study of hydrocarbon effect on a urea SCR was also performed and introduced in reference [110,112]. Toluene was used as a representative hydrocarbon and the experimental work was carried out on a bench reactor system. Hydrocarbon inhibition of the NO oxidation, hydrocarbon storage, and hydrocarbon effect on the SCR reactions were studied. It was concluded that the toluene adsorbed on the catalyst sites has 
inhibition effects on SCR reactions. More effects were observed under lower temperatures because of the greater toluene adsorption which blocked the active SCR sites. A higher ammonia oxidation rate was observed in the presence of toluene at temperatures higher than $350^{\circ} \mathrm{C}$. A model describing hydrocarbon storage on the SCR and the hydrocarbon effect on $\mathrm{NO}$ oxidation and $\mathrm{NO}_{2}$ dissociation was developed, and the model prediction was validated against the experimental data. The hydrocarbon poisoning effect on SCR performance was confirmed for both ULSD and biodiesel through experiments carried out in both a bench reactor and an engine test cell [109].

The influence of hydrocarbon storage on a Fe-zeolite and a Cu-zeolite SCR was studied in both a bench reactor and an engine test cell [113]. It was reported that both Fe-zeolite and Cu-zeolite SCR can store hydrocarbons up to $21 \mathrm{~g} / \mathrm{L}$ at a temperature of $200^{\circ} \mathrm{C}$. A SCR being exposed to a temperature ramp after storing excessive hydrocarbons may cause irreversible thermal damage to the catalyst because of the exotherm resulting from the oxidation of stored hydrocarbons. A Cu-zeolite SCR formulation with less hydrocarbon storage capacity can avoid the high exotherm from oxidation of stored hydrocarbons [113].

The HC poisoning effect on the SCR performance and modeling of the HC effects are still open research areas. Effectively designed experiments are required to study the mechanisms of $\mathrm{HC}$ inhibition on SCR reactions. On the modeling aspects, only models of $\mathrm{HC}$ storage and the $\mathrm{HC}$ effect on $\mathrm{NO}$ oxidation are available from the literature. Engine experimental data shows low engine out $\mathrm{HC}$ levels (about 100 ppmC) and the $\mathrm{HC}$ conversion efficiency across the DOC and CPF is close to $100 \%$. Therefore, the $\mathrm{HC}$ level at SCR inlet is low when the SCR is located after the DOC and CPF in heavy-duty diesel engine aftertreatment systems. The chance of seeing a significant level of hydrocarbons at the SCR inlet only happens under multiple system failures including DOC and CPF failure and engine injector failure. As a result, it is not important to study the hydrocarbon poisoning effects on SCR kinetics under normal operating conditions for heavy-duty diesel applications. 


\subsubsection{SCR Thermal Aging Effects}

The SCR is periodically exposed to high temperatures (> $550 \mathrm{C}$ ) during the CPF active regeneration conditions. The SCR capability of reducing $\mathrm{NO}_{x}$ may deteriorate over time as a result of thermal deactivation. The thermal durability and thermal aging effect of the Cu-zeolite SCR were studied [44,114]. It has been shown from the studies that extremely high temperatures (800 - $900 \mathrm{C}$ ) can permanently damage the SCR catalyst, but the application-relevant aging conditions have a small effect on the performance of the Cu-zeolite SCR. A 5-10\% of reduction in $\mathrm{NO}_{x}$ conversion efficiency was reported from a vehicle aged SCR at the end of the vehicle life [114].

The SCR performance under different levels of hydrothermal aging (also known as steam aging or steam treatment) conditions was evaluated. Compared to a de-greened catalyst, the experimental results show that progressive hydrothermal aging of the $\mathrm{Cu}$ zeolite SCR up to $850^{\circ} \mathrm{C}$ for 10 hours leads to $[44,114]$ :

1. limited $(<5 \%)$ increase in $\mathrm{NO}_{x}$ conversion below $400^{\circ} \mathrm{C}$,

2. significant decrease in $\mathrm{NO}_{x}$ conversion above $450^{\circ} \mathrm{C}\left(>20 \%\right.$ at $\left.500^{\circ} \mathrm{C}\right)$,

3. increase in $\mathrm{NO}$ conversion to $\mathrm{NO}_{2}$ throughout the reaction temperature range,

4. increase in $\mathrm{NH}_{3}$ oxidation and selectivity to $\mathrm{NO}_{x}$, and

5. negligible change in $\mathrm{NH}_{3}$ storage capacity.

Progressive hydrothermal aging of the Cu-zeolite SCR up to $900^{\circ} \mathrm{C}$ for 10 hours leads to $[44,114]$ :

1. substantial loss of $\mathrm{NO}_{\mathrm{x}}$ conversion,

2. substantial degradation of $\mathrm{NO}$ conversion to $\mathrm{NO}_{2}$,

3. substantial degradation of $\mathrm{NH}_{3}$ oxidation, and

4. substantial degradation of $\mathrm{NH}_{3}$ storage capacity. This degradation is irreversible because of the SCR structure collapsing caused by extreme hydrothermal aging. 
A study of the thermal durability of the SCR catalyst which is used in GM light-duty trucks was in traduced in reference [115]. The performance of the SCR catalysts aged in lab reactors, lab oven, and on vehicles were compared. Both lab reactor aging under $850^{\circ} \mathrm{C}$ for 12 hours and oven aging under $850^{\circ} \mathrm{C}$ for 54 hours can simulate the performance of 135,000 mile vehicle aged catalysts. This study confirmed the aging effect discussed above, and damage equations for a reactor aged SCR were developed and are given in Figure 2.4. The functions are water content and catalyst formulation dependent. It was also concluded that water content can accelerate the SCR thermal aging by comparing lab reactor aging and oven aging [115]. Modeling of the thermal aging effect is ongoing and publications about the modeling studies were planned, but no results have been published.

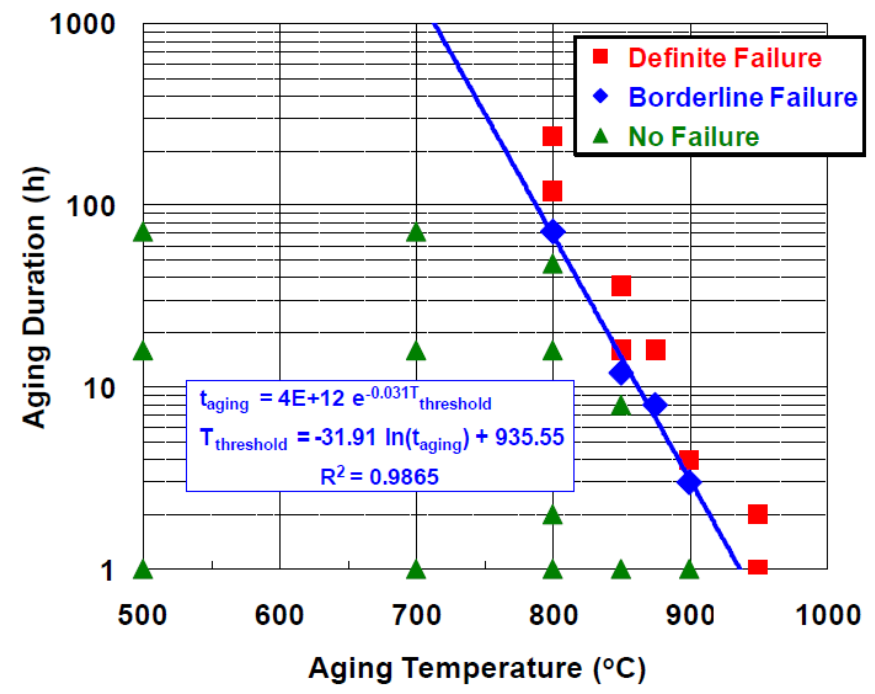

Figure 2.4: Damage equations for reactor aging of $\mathrm{Cu}$-chabazite SCR with space velocity of $30,000 \mathrm{hr}^{-1}$ and $5 \% \mathrm{H}_{2} \mathrm{O}$ [115]

\subsubsection{Maldistribution Effects}

It has been well known that the $\mathrm{NH}_{3}$ slip will be present at the SCR outlet under the conditions of the ANR being higher than then stoichiometric ratio. With an ANR lower or equal to the stoichiometric ratio, the nonuniformly distributed $\mathrm{NH}_{3}$ caused by poor mixing of the injected urea and exhaust flow may result in higher ANR at certain SCR inlet 
areas which then forms $\mathrm{NH}_{3}$ slip. The effects on the $\mathrm{NH}_{3}$ maldistribution on the SCR performance cannot be neglected in engine applications.

The uniformity of the interested parameter at the SCR inlet can be represented by the uniformity index (UI) which ranges from 0 to 1. A UI of one represents perfectly uniform distribution. The uniformity of both $\mathrm{NO}_{x}$ and $\mathrm{NH}_{3}$ at the SCR inlet were discussed. The calculation of UI is given in Equation 2.31.

$$
U I=1-\frac{\sum_{i}\left|v_{i}-v_{\text {avg }}\right| \cdot A_{i}}{2 v_{\text {avg }} A_{\text {total }}}
$$

Where, $v_{i}$ is the interested local parameter at the $i$ th cell of the inlet face which the area of $A_{i} . v_{\text {avg }}$ is the average of all $v_{i}$ values. $A_{\text {total }}$ is the inlet area of the catalyst.

The effects of the uniformity indices on the SCR performance were studied in reference [116] through simulation based on a vanadium SCR model which was divided into several parallel zones to enable the ability of using non - uniform input. A relationship between the simulated $\mathrm{NO}_{x}$ conversion efficiency and $\mathrm{NH}_{3}$ slip as a function of the ANR uniformity is shown in Figure 2.5. The results showed reduction in $\mathrm{NO}_{x}$ conversion and increase in $\mathrm{NH}_{3}$ slip with decrease of ANR uniformity.

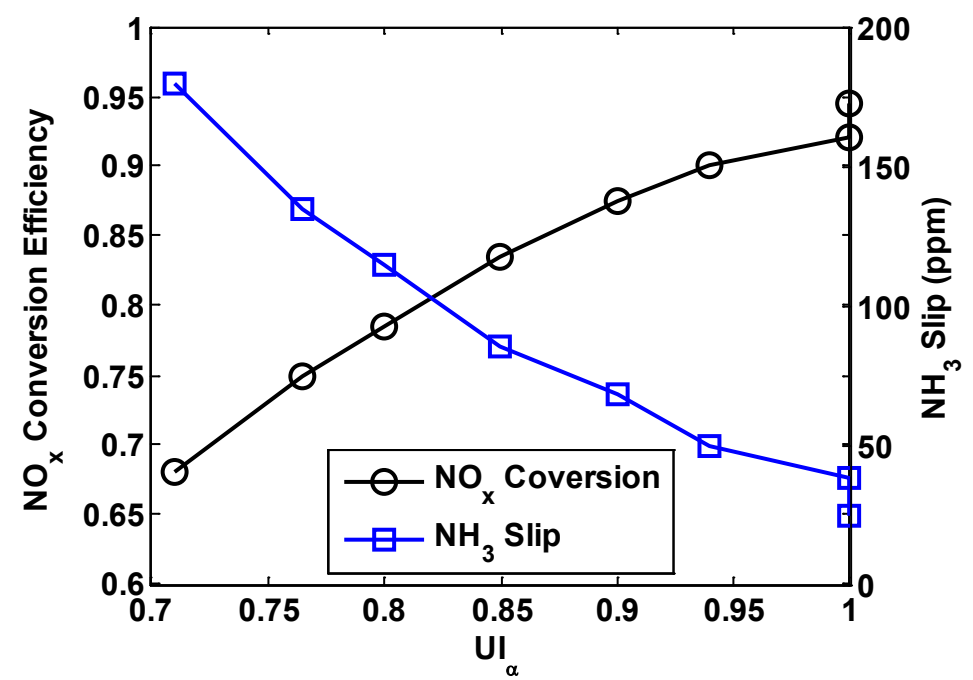

Figure 2.5: $\mathrm{NO}_{x}$ conversion efficiency and $\mathrm{NH}_{3}$ slip as a function ANR uniformity [116] 
Another simulation study of the ANR uniformity effects on the " $\mathrm{NH}_{3}$ slip limited $\mathrm{NO}_{\mathrm{x}}$ conversion efficiency" of the SCR for both steady state and transient conditions was presented in reference [117]. Several copies of a SCR model calibrated to Cu-zeolite SCR data were setup to run in parallel with different inlet ANR values. A probability distribution function (PDF) was used to generate the different ANRs based on beta distribution for a certain UI. The simulations were conducted for different steady state engine operating conditions as well as the heavy-duty FTP and SET emission test cycles to find the required $\mathrm{UI}$ for achieving the optimal SCR performance. The results showed that the UI requirements are different for different engine operating conditions with less critical UI requirements for moderate and high temperatures. It also concluded that higher UI was required for the heavy-duty FTP cycle than the SET cycle. The uniformity of the ANR at the SCR inlet can be improved by increasing urea injection performance, better mixer design, more efficient system configuration, etc [118-121].

The nonuniformity of the exhaust flow going into the SCR is another factor that might affect the SCR performance because of the nonuniformly distributed space velocity for different regions of the catalyst. The flow nonuniformity effects were studied by a few researchers mainly through Computational Fluid Dynamics (CFD) analysis $[120,122,123]$. It was not investigated by most of the SCR modeling studies.

\subsection{Summary}

Due to the standards of the 2010 emission regulations, aftertreatment systems with a DOC, CPF and urea-SCR are used for heavy-duty diesel engines. Because of the prior performance of the exchange metal based SCR catalysts, the Fe-and Cu-zeolite SCR are used for $\mathrm{NO}_{x}$ reduction. Flow reactor experimental studies provided opportunities to study the catalyst performance under wide a range of conditions and for various catalyst kinetic properties. At the same time, engine experimental studies under steady state and transient conditions were necessary to understand the SCR performance under complex engine exhaust conditions. 
Efforts were carried out for developing SCR kinetic models. The Eley-Rideal mechanism which assumes only one of the reactants gets adsorbed on the catalytic surface and reacts with other reactants is typically used to model the reaction mechanisms over the SCR catalyst. $\mathrm{NH}_{3}$ is assumed to be the only species being adsorbed on the catalyst and the stored $\mathrm{NH}_{3}$ reacts with other species in the surface phase which is transported from species in the bulk flow (the gas phase). External mass transfer between species in gas and surface phases was considered in published high fidelity models. The reduced order models neglected the mass transfer process and allow species in the gas phase to react with the stored $\mathrm{NH}_{3}$. Limitations of internal mass transfer and other mass transfer processes on the SCR performance are being studied. Most of the SCR models have only one type of $\mathrm{NH}_{3}$ storage site which stores $\mathrm{NH}_{3}$ and supports all SCR reactions. Adding a second site improves the $\mathrm{NH}_{3}$ adsorption and desorption performance of the model but this also adds more parameters to identify. In this study, 2-site modeling approach was adopted to simulate both species concentrations and the $\mathrm{NH}_{3}$ storage.

The major global SCR reactions included in the published models were similar. The detailed SCR reaction mechanisms are still under investigation. Urea decomposition, solid deposit formation from injected urea and nitrates formation under SCR reaction conditions were considered by a few models but excluded by most of the studies. SCR models were commonly calibrated against reactor data and validated against engine experimental data. SCR models calibrated to engine experimental data are important for on-vehicle model based control strategy and OBD development. In this study, a global reaction mechanism was selected to represent the reaction system for the Cu-zeolite catalyst. The SCR model was developed to simulate the catalyst performance for engine exhaust conditions.

The factors affecting the SCR performance were studied. Those factors include HC poisoning, thermal aging, etc. For heavy-duty diesel applications, the chance for the SCR to see high concentrations of $\mathrm{HC}$ is limited. Modeling efforts are being carried out to include these effects. Modeling the $\mathrm{HC}$ effects is problematic because a single $\mathrm{HC}$ type cannot represent the complex HC compounds in the diesel exhaust. The thermal aging effects on the SCR performance was studied through reactor experiments. 
Modeling results of the thermal aging effects on the SCR performance and the kinetics have not been reported. The $\mathrm{NH}_{3}$ distribution at the SCR inlet is not uniform in engine environments. The $\mathrm{NH}_{3}$ maldistribution needs to be quantified through SCR engine experiments. The $\mathrm{NH}_{3}$ maldistribution effects on the SCR performance were studied mainly through model simulation. It is considered to be one of the factors that causing the different SCR performance between reactor and engine testing environments. Coupled experimental and modeling studies were performed in this thesis research to determine the $\mathrm{SCR}$ inlet $\mathrm{NH}_{3}$ maldistribution profiles and to investigate its effects on the catalyst performance and the kinetics. A modeling study of the thermal aging effects on the SCR performance and kinetics is also presented in this thesis. 


\section{Chapter 3.}

\section{Experimental Setup and Test Procedures}

This chapter presents the engine test cell setup for both steady-state and transient SCR engine experiments, the reactor experimental setup for both flow reactor and Spaci-IR experiments including the test matrixes and test procedures. The SCR maldistribution experiments are also explained.

\subsection{Engine Test Cell Setup}

The Michigan Tech diesel engine test cell was designed for acquiring diesel aftertreatment system experimental data for the DOC, CPF, and SCR. A picture of the test cell is shown in Figure 3.1. Various parameters affecting the performance of the aftertreatment system can be measured and recorded during an experiment.

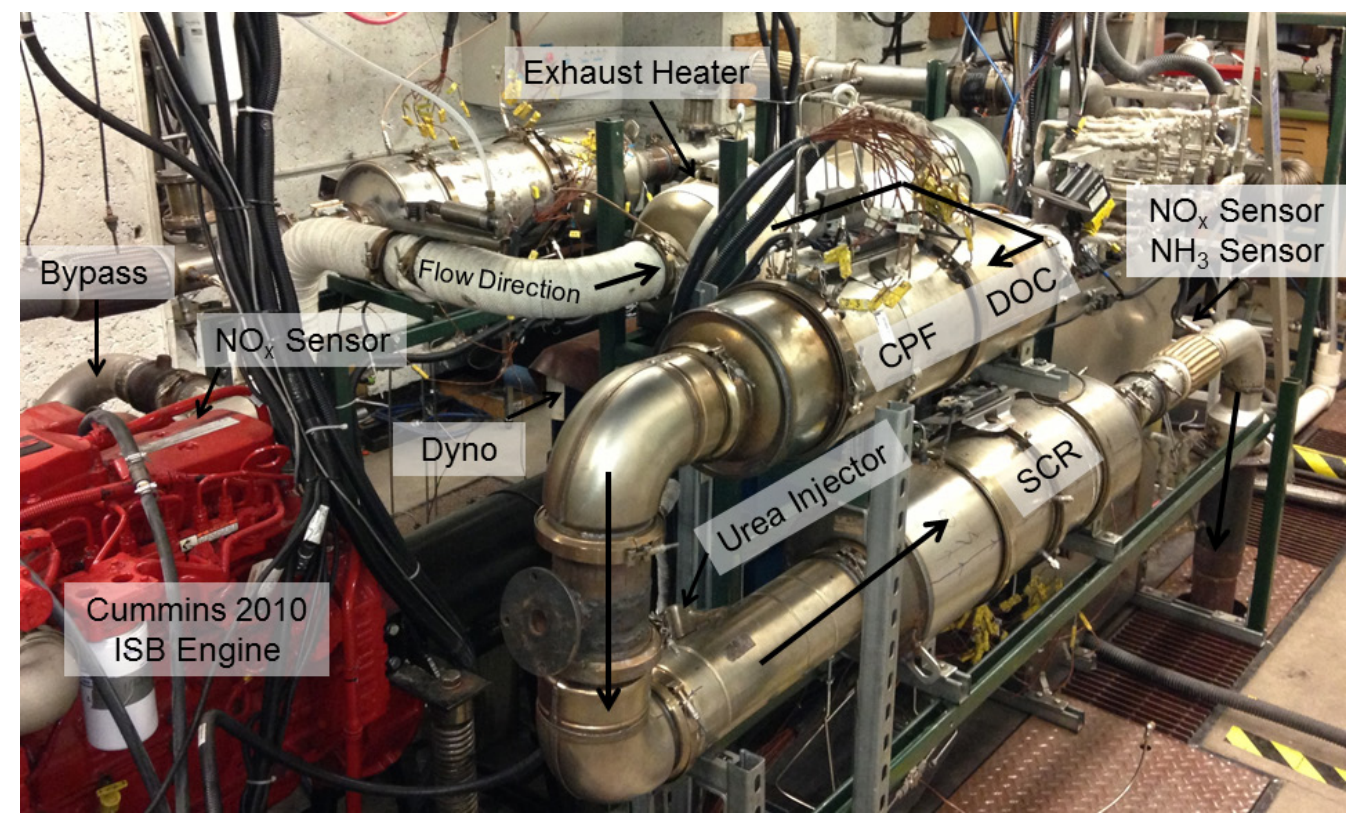

Figure 3.1: Picture of the diesel engine test cell at MTU 


\subsection{Engine, Fuel and Aftertreatment System}

\subsubsection{Engine and Dynamometer}

A 2010 Cummins ISB $224 \mathrm{~kW}$ (300 hp) engine which meets the U.S. EPA 2010 emission regulations is used in the study. The specifications of the engine are given in Table 3.1. The dynamometer coupled to the engine is an eddy current dynamometer from Eaton Corp., with a maximum power capacity of $370 \mathrm{~kW}(500 \mathrm{hp})$ from 1700 to $7000 \mathrm{rpm}$. The speed and torque of the engine is controlled by a Digalog Model 1022A controller which is set to 'Speed Control' mode. It regulates the engine speed by regulating dynamometer speed at certain desired values. The engine torque is controlled by varying the fuel flow rate supplied to the engine through a manually controlled rheostat. In-cylinder fuel injectors were used for injecting diesel fuel into the exhaust to initiate the CPF active regeneration process.

Table 3.1: Specifications of 2010 Cummins ISB engine

\begin{tabular}{|c|c|}
\hline Model & Cummins ISB 224 kW (300 hp) \\
\hline Year of Manufacture & 2010 \\
\hline Cylinders & 6 \\
\hline Bore \& Stroke & $407 \times 124 \mathrm{~mm}$ \\
\hline Displacement & Turbocharged \\
\hline Aspiration & Cummins Charge Air Cooler \\
\hline Aftercooling & Variable Geometry Turbocharger (VGT) \\
\hline Turbocharger & 2600 RPM and 224 kW \\
\hline Rate Speed and Power & 895 N.m @1600 RPM \\
\hline Peak Torque & Electronically controlled and cooled \\
\hline EGR system &
\end{tabular}

\subsubsection{Fuel Properties}

Two batches of ultra-low sulfur diesel (ULSD) were used for SCR testing. The fuel was analyzed in the Cummins lab to determine the chemical properties. The fuel properties are shown in Table 3.2. 
Table 3.2: Fuel properties for the ULSD used for SCR testing

\begin{tabular}{|l|r|r|}
\cline { 2 - 3 } \multicolumn{1}{c|}{} & ULSD -1 & ULSD -2 \\
\hline API. Gravity at $15.6^{\circ} \mathrm{C}$ & 35.6 & 33.4 \\
\hline SP. Gravity at $15.6^{\circ} \mathrm{C}$ & 0.847 & 0.858 \\
\hline Viscosity at $40^{\circ} \mathrm{C}$ & 2.290 & 2.609 \\
\hline Total Sulfur $(\mathrm{ppm})$ & 7 & 12 \\
\hline Initial Boiling Point $\left({ }^{\circ} \mathrm{C}\right)$ & 168 & 172 \\
\hline Final Boiling Point $\left({ }^{\circ} \mathrm{C}\right)$ & 340 & 359 \\
\hline ICP for metals $(\mathrm{ppm})$ & $<1$ & $<1$ \\
\hline Water Content $(\mathrm{ppm})$ & 92 & 348 \\
\hline IR for \% Biodiesel & 0 & $<0.1$ \\
\hline Higher Heating Value $(\mathrm{MJ} / \mathrm{kg})$ & 45.60 & 45.21 \\
\hline Lower Heating Value $(\mathrm{MJ} / \mathrm{kg})$ & 42.80 & 42.55 \\
\hline
\end{tabular}

\subsubsection{Engine Aftertreatment System}

The engine is equipped with in-cylinder fuel injector for active CPF regeneration and a full aftertreatment system including DOC, CPF, SCR, and AMOX. Each catalyst is mounted in a stainless steel can that can be removed from the exhaust line. The specifications of each catalyst are shown in Table 3.3. There are two SCR bricks with the length of 6 inches each.

The aftertreatment system was de-greened before running tests and collecting data, because the performance of the catalysts may change during the first period of operation. The de-greening process was carried out based on the information provided by Cummins. The de-green conditions consisted of 12 engine operating points, with the engine exhaust running through the aftertreatment system for 1 hour for each engine operating condition. The engine operating points were provided by Cummins and are given in Table 3.4 
Table 3.3: Specifications of the catalysts in 2010 Cummins ISB engine aftertreatment system

\begin{tabular}{|c|c|c|c|c|}
\hline & DOC & CPF & SCR & AMOX \\
\hline Material & Cordierite & Cordierite & Cordierite & Cordierite \\
\hline Diameter (inch) & 9.50 & 9.50 & 10.50 & 10.50 \\
\hline Length (inch) & 4 & 12 & $12^{*}$ & 4 \\
\hline Cell Geometry & Square & Square & Square & Square \\
\hline Total Volume (L) & 4.65 & 13.94 & 17.03 & 5.68 \\
\hline Open Volume (L) & 3.92 & NA & 14.41 & 4.80 \\
\hline Cell Density /in ${ }^{2}$ & 400 & 200 & 400 & 400 \\
\hline Cell Width (mil) & 46 & 59 & 46 & 46 \\
\hline Filtration Area (in $\left.{ }^{3}\right)$ & NA & $1.99 \mathrm{e} 4$ & NA & NA \\
\hline Open Frontal Area (in $\left.{ }^{2}\right)$ & 60.00 & 24.43 & 73.29 & 73.29 \\
\hline Channel Wall Thickness (mil) & 4 & 12 & 4 & 4 \\
\hline Wall density (g/cm ${ }^{3}$ ) & 1.2 & 1.2 & 1.2 & 1.2 \\
\hline Porosity & $35 \%$ & $59 \%$ & $35 \%$ & $35 \%$ \\
\hline Mean Pore Size $(\mu m)$ & NA & 15 & NA & NA \\
\hline Number of inlet cells & $2.84 \mathrm{e} 4$ & $0.71 \mathrm{e} 4$ & $3.46 \mathrm{e} 4$ & $3.46 \mathrm{e} 4$ \\
\hline * There are 2 6-inch long SCR bricks with 1.5 inches space in between
\end{tabular}

Table 3.4: Engine operating points for de-greening the aftertreatment system

\begin{tabular}{|c|c|c|c|c|c|c|}
\hline $\begin{array}{c}\text { Degreen } \\
\text { Point }\end{array}$ & Speed & Load & $\begin{array}{c}\text { Air Mass } \\
\text { Flowrate }\end{array}$ & $\begin{array}{c}\text { Fuel Mass } \\
\text { Flowrate } \\
(\mathrm{kg} / \mathrm{min})\end{array}$ & $\begin{array}{c}\text { Exhaust Mass } \\
\text { Flowrate } \\
(\mathrm{kg} / \mathrm{min})\end{array}$ & $\begin{array}{c}\text { SCR Inlet } \\
\text { Temperature }\end{array}$ \\
\hline & $(\mathrm{rpm})$ & $(\mathrm{N} \cdot \mathrm{m})$ & $(\mathrm{kg} / \mathrm{min})$ & 0.21 & 6.90 & 265 \\
\hline 1 & 1614 & 215 & 6.69 & 0.32 & 8.37 & 362 \\
\hline 2 & 1615 & 453 & 8.05 & 0.39 & 9.56 & 444 \\
\hline 3 & 1594 & 670 & 9.17 & 0.51 & 10.9 & 485 \\
\hline 4 & 1594 & 867 & 10.4 & 0.21 & 9.54 & 287 \\
\hline 5 & 1975 & 220 & 9.33 & 0.35 & 10.6 & 357 \\
\hline 6 & 1975 & 415 & 10.3 & 0.46 & 13.3 & 412 \\
\hline 7 & 1975 & 665 & 12.9 & 0.63 & 16.1 & 437 \\
\hline 8 & 1975 & 875 & 15.4 & 0.27 & 12.9 & 290 \\
\hline 9 & 2327 & 229 & 12.6 & 0.43 & 14.5 & 357 \\
\hline 10 & 2327 & 440 & 14.0 & 0.60 & 17.7 & 399 \\
\hline 11 & 2327 & 665 & 17.1 & 0.6 & 444 \\
\hline 12 & 2327 & 885 & 19.2 & 0.77 & 19.9 & \\
\hline
\end{tabular}




\subsubsection{Exhaust Heater}

A $25 \mathrm{~kW}$ exhaust heater designed and manufactured by Watlow Electric Manufacturing Company was integrated into the ISB engine test cell for the purpose of expanding the testing temperature range and improved steady-state temperature control. The heater was installed before the DOC in order to raise the temperature of exhaust flow going through the entire aftertreatment system. It enables the aftertreatment catalysts to be evaluated under different temperatures without changing the exhaust flow rate and engine operating conditions. The temperature effects on the catalysts performance can be isolated by utilizing the heater. Since the heater outlet temperature is controlled by an independent PID controller, it is able to maintain the exhaust temperature of the aftertreatment system more constant regardless of the slight changes in engine operating conditions. The heater capability of heating up the exhaust is shown in Figure 3.2. With an exhaust mass flow rate of $4.45 \mathrm{~kg} / \mathrm{min}$, the temperature of the exhaust can be increased $250^{\circ} \mathrm{C}$ by the heater. For an exhaust mass flow rate of $11.7 \mathrm{~kg} / \mathrm{min}$, a temperature increase of $125^{\circ} \mathrm{C}$ can be achieved by the heater.

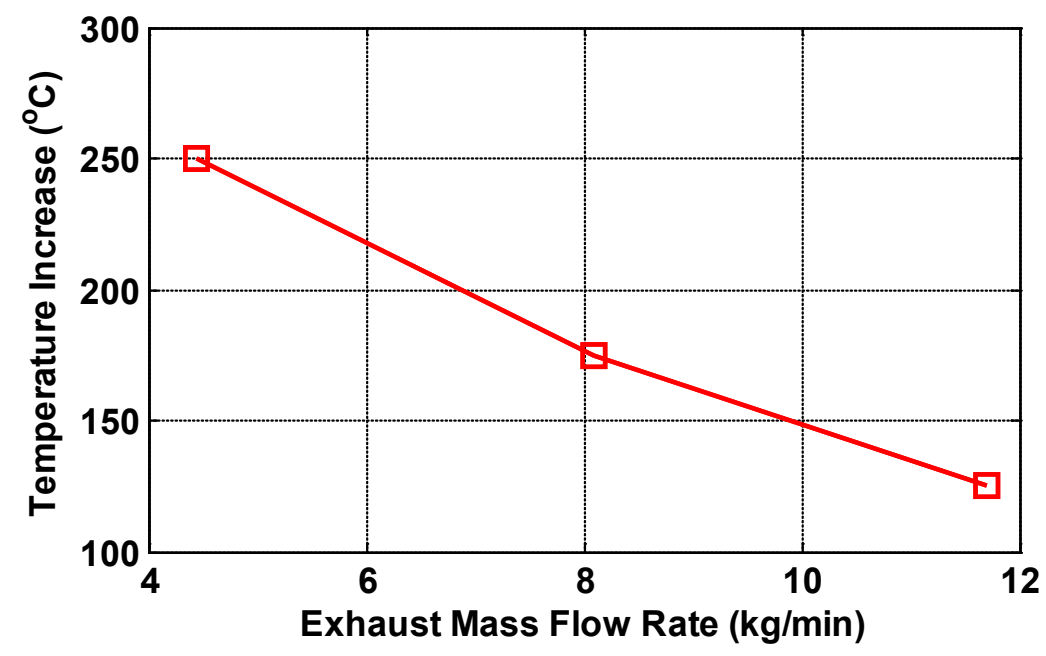

Figure 3.2: The heating capability of the heater as a function of the exhaust mass flow rate going through the heater 
The effects of the heater on the emissions were evaluated by taking emission measurements and PM samples under different heater operating conditions. The DOC inlet $\mathrm{NO}_{2} / \mathrm{NO}_{x}$ ratio affected by the heating element temperature of the heater is shown in Figure 3.3. The engine operating condition was maintained at $1300 \mathrm{rpm}$ and $240 \mathrm{~N} \cdot \mathrm{m}$. A DOC inlet $\mathrm{NO}_{2} / \mathrm{NO}_{x}$ ratio of $4.8 \%$ was observed with the heater off. After turning on the heater, the ratio keeps decreasing when the heating element temperature is increasing, meaning that more $\mathrm{NO}_{2}$ in the exhaust is oxidized through the heater as its temperature increases.

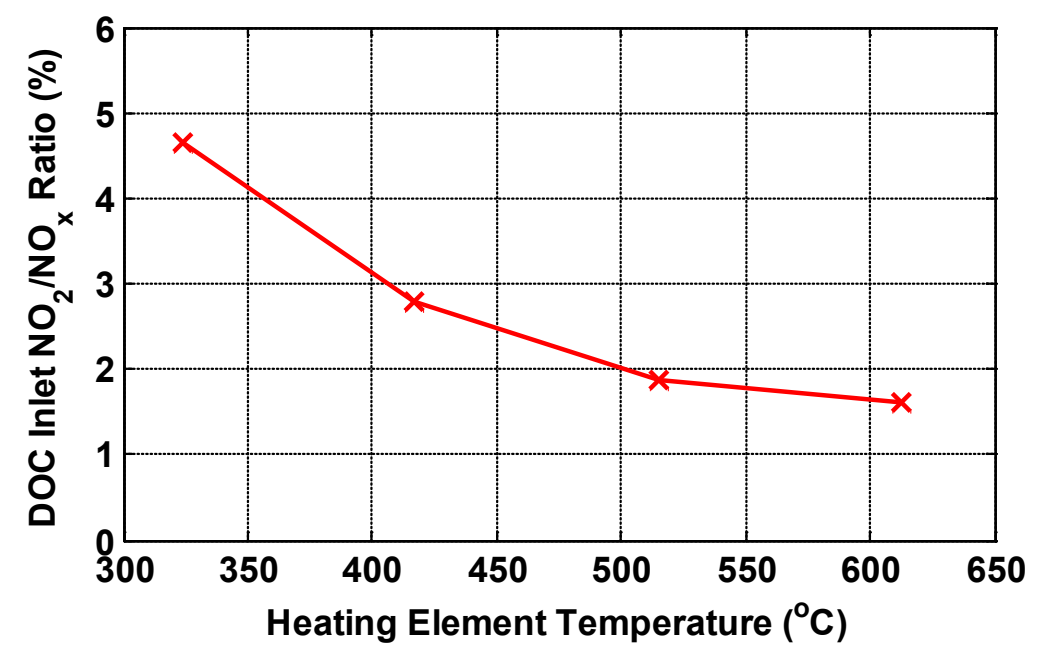

Figure 3.3: The $\mathrm{DOC}$ inlet $\mathrm{NO}_{2} / \mathrm{NO}_{\mathrm{x}}$ ratio as a function of the heating element temperature

Similarly, the DOC inlet PM concentrations as a function of the heating element temperature is given in Figure 3.4. The engine operating conditions for the test was maintained at $2500 \mathrm{rpm}$ and $245 \mathrm{~N} \cdot \mathrm{m}$. The error bars in this figure represent the plus and minus the standard error for the PM samples taken. A decreasing trend can be observed in the PM concentration, showing that the heater oxidizes more PM at higher temperatures. 


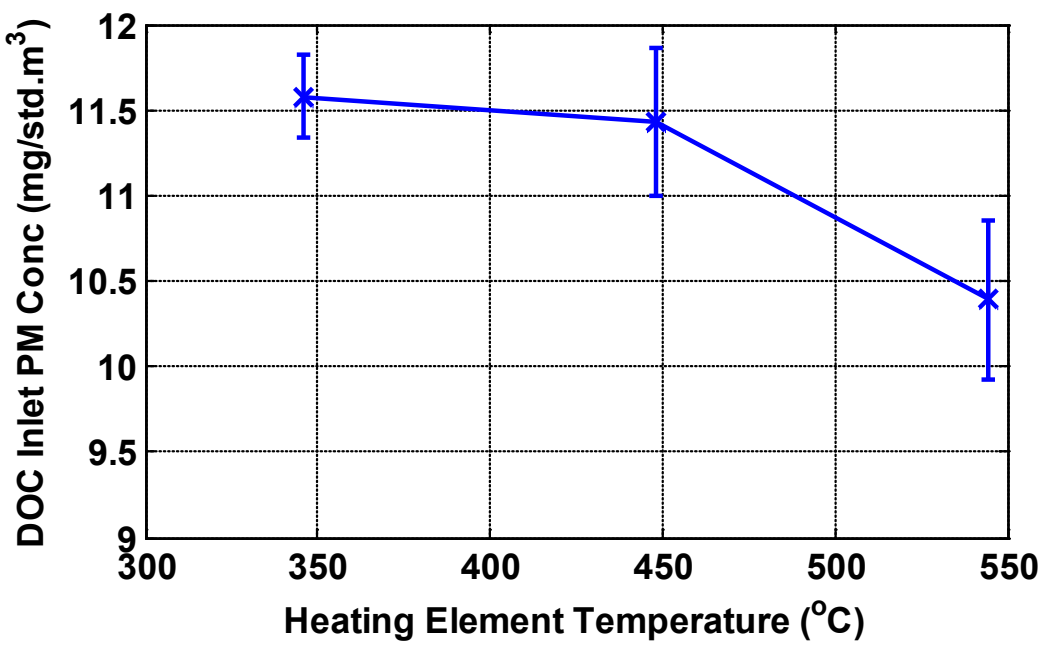

Figure 3.4: The DOC inlet PM concentration as a function of the heating element temperature

\subsubsection{Test Cell Layout}

The layout of the engine and aftertreatment system is shown in Figure 3.5. The engine exhaust is routed through a four-inch diameter exhaust pipe. There are two paths for the exhaust to go through. One path called baseline leads directly to the building exhaust ventilation system without passing through the aftertreatment system. The other path is called trapline which has full aftertreatment system components. Exhaust flow can be controlled to go through either path by opening and closing the corresponding pneumatic valves located at the junction of the two paths. When it goes to the trapline, the first component is the DOC with an exhaust mixer in front to improve the mixing of the fuel and exhaust gas going into the DOC during the active regeneration process. The $25 \mathrm{~kW}$ exhaust heater described in Section 3.2.4 was installed before the DOC to raise or maintain the exhaust temperature when necessary. The next component is the CPF, followed by a decomposition tube with the DEF injector mounted on it, and then the SCR catalysts. There is a mixer in between the DEF injector and SCR inlet face to improve the mixing of the injected DEF droplets and the exhaust gas stream. Another mixer is installed after the SCR bricks, before the production $\mathrm{NO}_{x}$ sensor, ammonia sensor, and the emission sampling probe. The purpose of this mixer is to make sure that the exhaust 
gas is uniform and the sensors and analyzers are measuring the same gas components. The AMOX was removed from the trap line for the purposes of measuring ammonia slip downstream of the SCR during DEF injection. The distance between the SCR downstream sampling location and the SCR outlet face as well as the mixer improve the mixing of the exhaust gas being measured by multiple instruments.

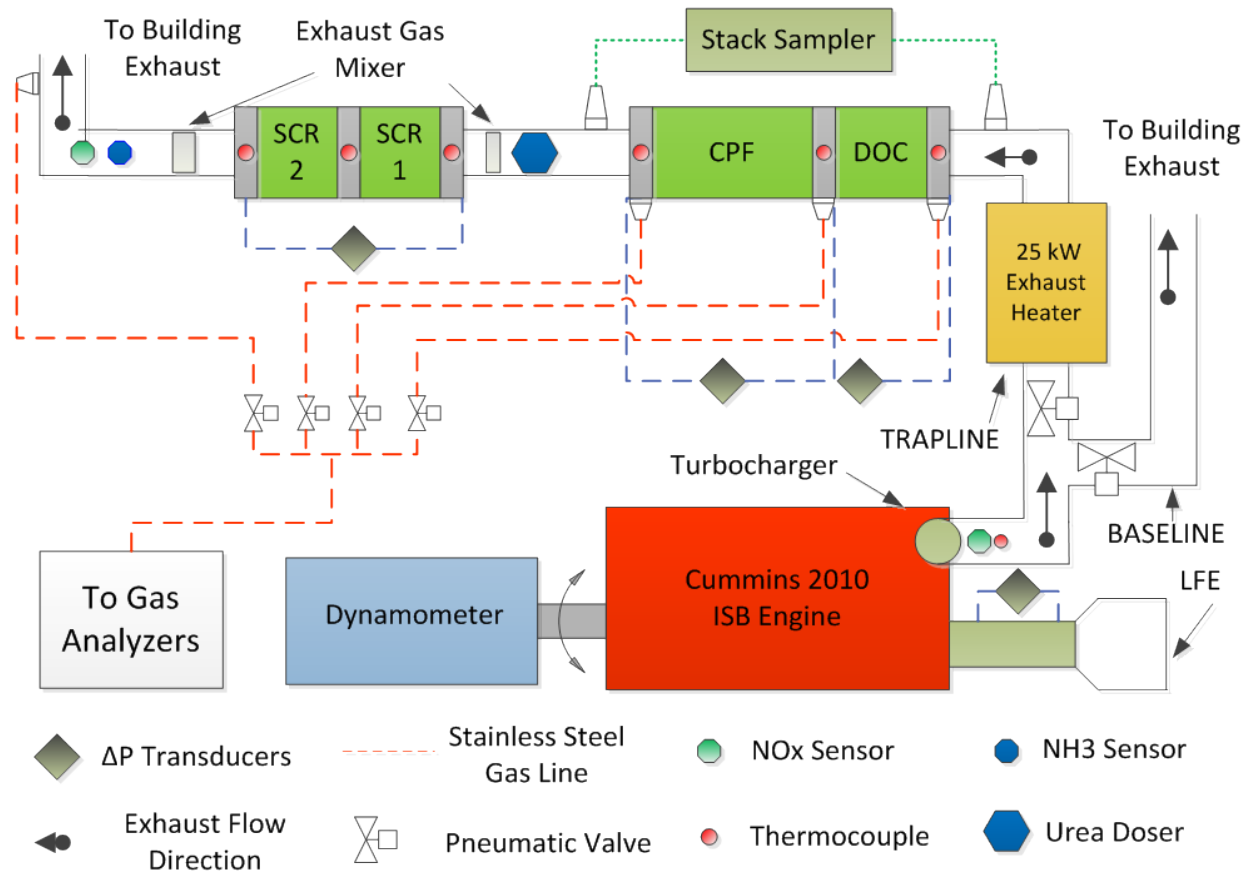

Figure 3.5: Schematic of the ISB engine test cell setup with instrumentation

\subsection{Measurements and Data Acquisition System}

\subsubsection{Temperature Measurement}

The catalysts performance is highly dependent on exhaust temperatures. Temperatures at different locations throughout the exhaust pipes and in the catalyst substrates are measured using type $\mathrm{K}$ thermocouples provided by Watlow. The thermocouples used in the substrates and exhaust pipes are listed in Table 3.5. 
Table 3.5: Specifications of thermocouples used in ISB aftertreatment system

\begin{tabular}{|c|c|c|c|c|c|c|}
\hline Location & Type & Diameter & Length & Watlow Part \# & Body material & Quantity \\
\hline DOC & K & $0.020 "$ & $12 "$ & AX1078701 & Stainless Steel & 9 \\
\hline CPF & K & $0.032 "$ & $12 "$ & AX1078801 & Stainless Steel & 12 \\
\hline SCR & K & $0.020 "$ & $12 "$ & AX1078701 & Stainless Steel & 22 \\
\hline SCR & K & $0.020 "$ & $16 "$ & n/a & Stainless Steel & 4 \\
\hline Other & K & $0.125 "$ & $6 "$ & ACGF00Q060U4000 & Stainless Steel & 7 \\
\hline
\end{tabular}

The thermocouple layout in the DOC, CPF, and SCR substrates is carefully selected in order to determine both radial and axial temperature gradients within the substrates. The thermocouple layout in the DOC, CPF, and SCR are given in Figure 3.6, 3.7, and 3.8 respectively.

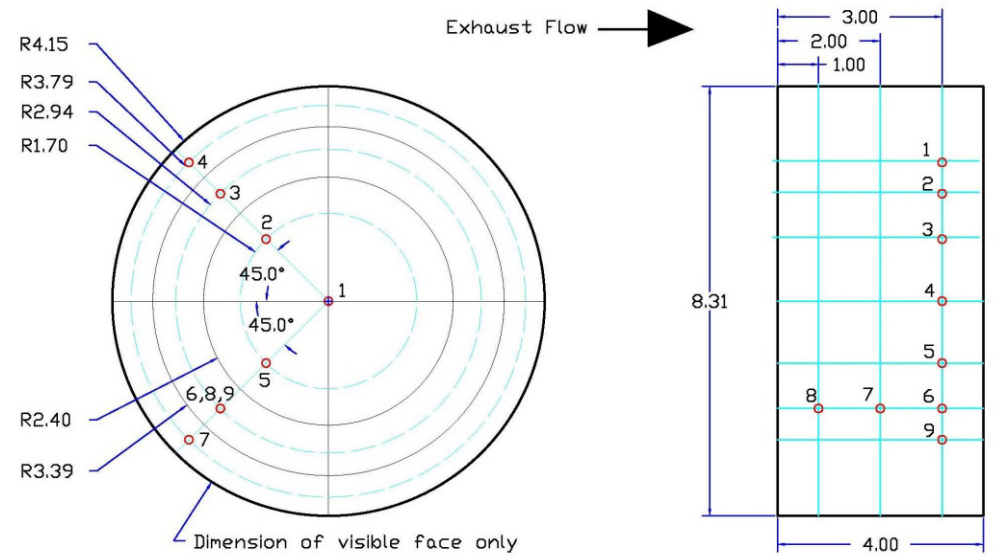

Figure 3.6 DOC thermocouple layout (Unit: inch)

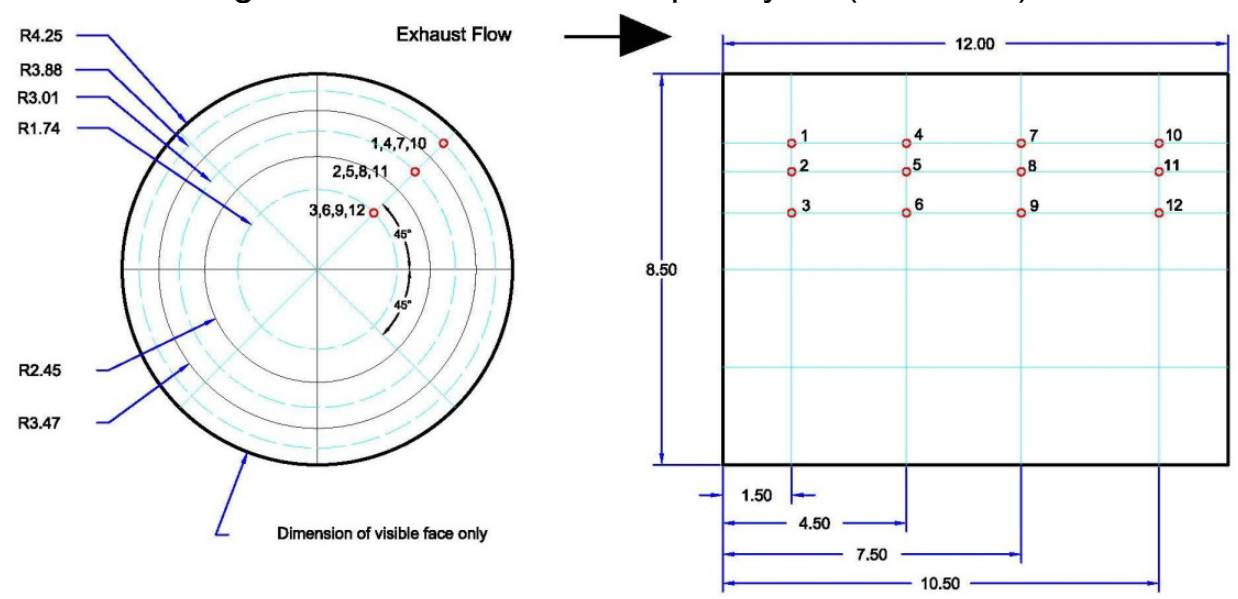

Figure 3.7: CPF thermocouple layout (Unit: inch) 


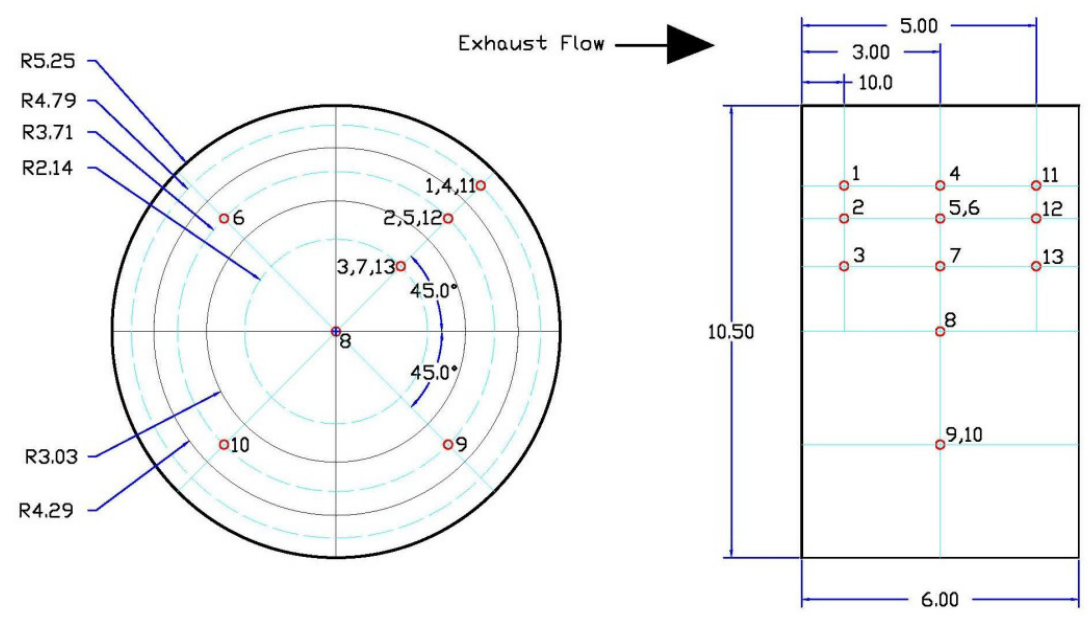

Figure 3.8: SCR thermocouple layout (Unit: inch)

\subsubsection{Pressure Measurement}

The barometric pressure of the test cell and the pressure drops across the Laminar Flow Element (LFE), DOC, CPF, and SCR substrates are continuously monitored by five different sensors. The barometric pressure is measured using a pressure transducer and the pressure drops are measured using four differential pressure transducers. The details about the sensors for differential pressures across the LFE, DOC, CPF, and the barometric pressure can be found in reference [124], while the information for the differential pressure across the SCR is given in Table 3.6.

Table 3.6: Specification of differential pressure transducers

\begin{tabular}{|c|c|c|c|c|c|c|}
\hline Parameter & Manufacture & Part No. & $\begin{array}{c}\text { Sensor } \\
\text { Type }\end{array}$ & Range & Unit & $\begin{array}{c}\text { Sensor } \\
\text { Output }\end{array}$ \\
\hline $\begin{array}{c}\text { DP across } \\
\text { SCR }\end{array}$ & $\begin{array}{c}\text { Omega } \\
\text { Engineering }\end{array}$ & $\begin{array}{c}\text { PX409- } \\
2.5 D W U 5 V\end{array}$ & $\begin{array}{c}\text { Differential } \\
\text { Pressure }\end{array}$ & $0.0 \sim 17.2$ & $\mathrm{kPa}$ & $\begin{array}{c}0 \sim 5 \\
\text { Vdc }\end{array}$ \\
\hline
\end{tabular}

\subsubsection{Exhaust Mass Flow Rate Measurement}

The exhaust mass flow rate is considered as the summation of engine intake air and fuel mass flow rates. The intake air standard volumetric flow rate is calculated based on the measured pressure drop generated by the intake air flow through the LFE. The standard 
volumetric flow rate is then converted to mass flow rate using standard density of the intake air under reference conditions ( $1 \mathrm{~atm}$ pressure and $20^{\circ} \mathrm{C}$ ). The fuel mass flow rate is measured by an AVL fuel system. The system measures the time for flowing $0.4 \mathrm{~kg}$ of fuel to the engine. By dividing the $0.4 \mathrm{~kg}$ of fuel by the time in seconds used for consuming the fuel gives the fuel mass flow rate in units of $\mathrm{kg} / \mathrm{sec}$. Adding the fuel mass flow rate to the intake air mass flow rate gives the exhaust mass flow rate. This measurement process and calculation equations are described in detail in reference [124].

\subsubsection{Gaseous Emissions}

The gaseous emissions are measured using two different analyzers and also by production sensors. One of the analyzers is an AVL AMA 4000 exhaust gas analyzer which is able to measure total hydrocarbon (THC), $\mathrm{O}_{2}, \mathrm{CO}_{2}, \mathrm{CO}$, and $\mathrm{NO}_{\mathrm{x}}$ (or $\mathrm{NO}$ ). Detailed information about the analyzer and calibration gases used for adjusting the analyzer can be found in reference [124]. The analyzer consists of a flame ionization detector (FID) for THC measurement, a paramagnetic analyzer for $\mathrm{O}_{2}$ measurement, an infra-red detector (IRD) for $\mathrm{CO}$ and $\mathrm{CO}_{2}$ measurement, and a chemiluminescence detector (CLD) for $\mathrm{NO}_{\mathrm{x}}$ and $\mathrm{NO}$ measurement. The CLD is able to measure both $\mathrm{NO}_{\mathrm{x}}$ and NO but it cannot measure the two concentrations at the same time, since it must be switched between $\mathrm{NO}_{\mathrm{x}}$ and $\mathrm{NO}$ measurement mode.

For the SCR tests, continuous $\mathrm{NO}, \mathrm{NO}_{2}$, and $\mathrm{NH}_{3}$ concentration measurements are required, so an Airsense ion molecule reaction mass spectrometer (IMR-MS) was set up for the SCR tests [125]. The MS is setup to measure $\mathrm{NO}, \mathrm{NO}_{2}, \mathrm{NH}_{3}$, and $\mathrm{O}_{2}$. The monitoring mass and the ion source for each measured species are given in Table 3.7. $\mathrm{N}_{2} \mathrm{O}$ measurement is also desired for the SCR tests, but because of the interference issue, accurate $\mathrm{N}_{2} \mathrm{O}$ measurement cannot be measured by the MS. 
Table 3.7: Monitoring mass and the ion source for each measured species

\begin{tabular}{|c|c|c|c|c|}
\hline \multicolumn{2}{|c|}{ Components } & $\begin{array}{c}\text { Detection levels in } \\
\text { ppb at 100 msec }\end{array}$ & $\begin{array}{c}\text { Monitoring } \\
\text { Mass (amu) }\end{array}$ & lonisatior \\
\hline $\mathrm{NH}_{3}$ & Ammonia & 120 & 17 & $\mathrm{Hg}$ \\
\hline $\mathrm{NO}$ & Nitrogen monoxide & 100 & 30 & $\mathrm{Hg}$ \\
\hline $\mathrm{NO}_{2}$ & Nitrogen dioxide & 50 & 46 & $\mathrm{Hg}$ \\
\hline $\mathrm{O}_{2}$ & Oxygen & $<5 \mathrm{ppm}$ & 33 & $\mathrm{Xe}$ \\
\hline
\end{tabular}

A list of calibration gases for calibrating the IMR-MS is given in Table 3.8. The IMR - MS start up and calibration procedures are given in Appendix $A$.

Table 3.8: Specifications of calibration gases for IMR-MS

\begin{tabular}{|c|c|c|c|c|}
\hline Component & Span gas & Concentration & Units & Accuracy \\
\hline $\mathrm{NH}_{3}$ & $\mathrm{NH}_{3}, \mathrm{~N}_{2}$ balance & 103.8 & $\mathrm{ppm}$ & $\pm 2 \%$ \\
\hline $\mathrm{NO}$ & $\mathrm{NO}_{2} \mathrm{~N}_{2}$ balance & 515.4 & $\mathrm{ppm}$ & $\pm 1 \%$ \\
\hline $\mathrm{NO}_{2}$ & $\mathrm{NO}_{2}$, Air balance & 99.05 & $\mathrm{ppm}$ & $\pm 2 \%$ \\
\hline $\mathrm{O}_{2}$ & $\mathrm{NO}_{2}$, Air balance & 20.9 & $\%$ & N/A \\
\hline
\end{tabular}

The stainless steel sampling lines connecting the analyzers and sampling locations in the aftertreatment system are heated to $190^{\circ} \mathrm{C}$ to keep exhaust gas at a temperature that prevents water from condensing and to minimize the other gases from adsorbing on the sampling lines.

There are two UniNOx sensors installed in the production aftertreatment system. They are mounted at the engine outlet and the SCR outlet for $\mathrm{NO}_{x}$ measurement. A picture of the $\mathrm{NO}_{\mathrm{x}}$ sensor is given in Figure 3.9. It consists of a NGK sensing element with a Continental control unit. A Delphi ammonia sensor shown in Figure 3.10 is installed at the SCR outlet for ammonia slip measurement. The specifications of the $\mathrm{NO}_{\mathrm{x}}$ and $\mathrm{NH}_{3}$ sensors are given in Table 3.9. Data transmission with both sensors is based on controller-area network (CAN) communication. 


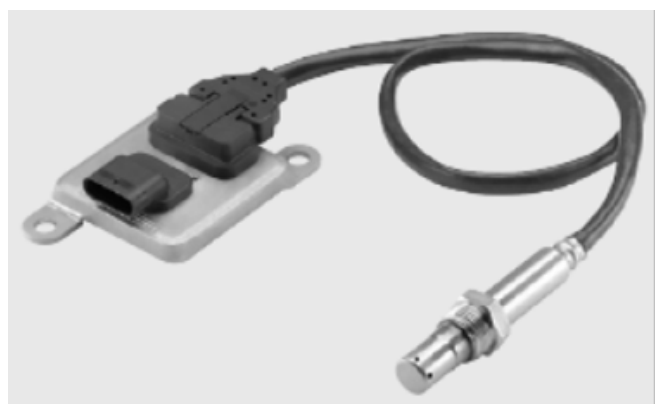

Figure 3.9: Production $\mathrm{NO}_{x}$ sensor

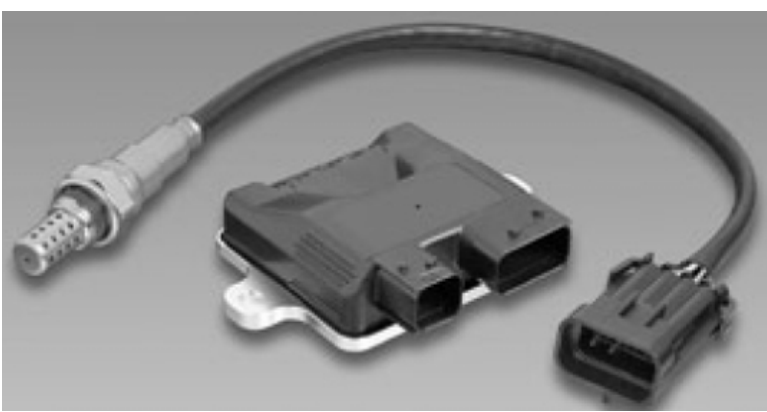

Figure 3.10: Delphi ammonia sensor

Table 3.9: Specifications of the $\mathrm{NO}_{x}$ sensor and $\mathrm{NH}_{3}$ sensor

\begin{tabular}{|c|c|c|c|c|c|}
\cline { 2 - 6 } \multicolumn{1}{c|}{} & Measurement & Range & Resolution & $\begin{array}{c}\text { Voltage } \\
\text { Range }\end{array}$ & $\begin{array}{c}\text { Operating } \\
\text { Temperature }\end{array}$ \\
\hline $\begin{array}{c}\mathrm{NO}_{\mathrm{x}} \\
\text { sensor }\end{array}$ & $\begin{array}{c}\mathrm{NO}_{\mathrm{x}} \\
\text { concentration }\end{array}$ & $\begin{array}{c}0-1500 \\
\mathrm{ppm}\end{array}$ & $0.1 \mathrm{ppm}$ & $12-32 \mathrm{~V}$ & $100-800^{\circ} \mathrm{C}$ \\
\hline $\begin{array}{c}\mathrm{NH}_{3} \\
\text { sensor }\end{array}$ & $\begin{array}{c}\mathrm{NH}_{3} \\
\text { concentration }\end{array}$ & $\begin{array}{c}0-1500 \\
\mathrm{ppm}\end{array}$ & $0.1 \mathrm{ppm}$ & $13.5-32 \mathrm{~V}$ & $200-500^{\circ} \mathrm{C}$ \\
\hline
\end{tabular}

\subsubsection{Particulate Matter}

The engine out particular matter (PM) concentration is measured during the test to provide data for potential CPF modeling work. The PM samples are taken by doing a hot sampling from the engine exhaust flow. The PM is sampled on a $47 \mathrm{~mm}$ diameter glass fiber filter using the stack-sampler. Detailed information about PM sample collection is available in references [124] and [126].

\subsubsection{Data Acquisition System}

National Instruments ( $\mathrm{NI}$ ) system was setup and a corresponding LabView program was created to collect temperature, pressure, engine speed/load data, and CAN messages. The hardware system consists of two NI Compact DAQ 8-Slot USB Chassis with multiple $\mathrm{NI}$ modules plugged in for collecting or generating different types of signals. The chassis is connected to the desktop computer through two separate USB cables. The detailed information about the modules being used is listed in Table 3.10. The CAN communication setup uses a separate USB-CAN interface which is connected to the desktop computer through another USB cable. 


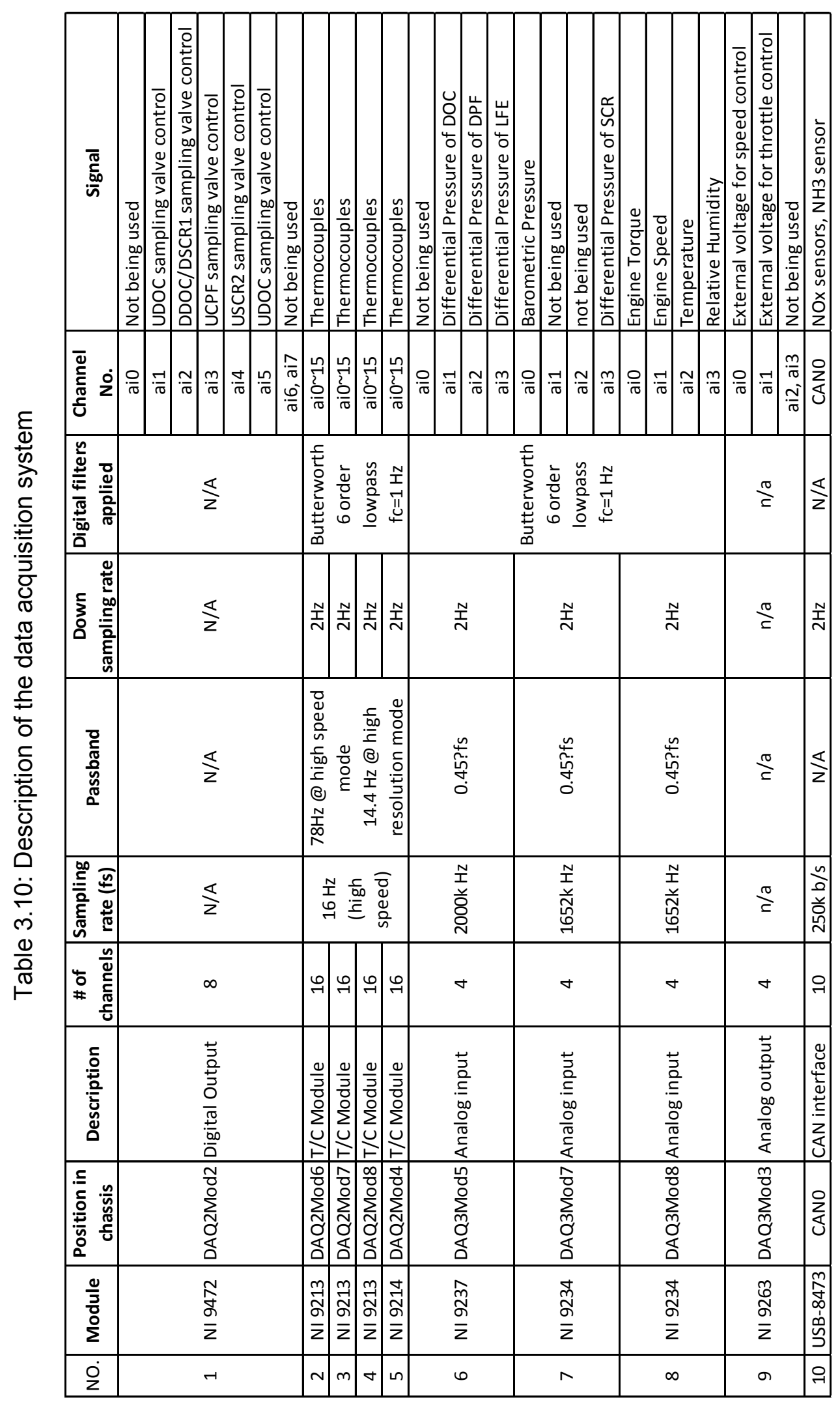




\subsection{Steady - State SCR Engine Tests}

The primary objective of the SCR engine tests is to acquire data suitable for SCR model calibration and state estimation strategy development. This requires measurements of exhaust temperature, $\mathrm{NO}, \mathrm{NO}_{2}$, and $\mathrm{NH}_{3}$ concentrations before and after the SCR for a variety of different steady-state and transient conditions.

SCR model calibration requires pre and post SCR measurements over a wide range of exhaust conditions (temperatures, flow rates, $\mathrm{NO}_{2} / \mathrm{NO}_{x}$ ratios, and $\mathrm{NH}_{3} / \mathrm{NO}_{x}$ ratios). Engine operating conditions were selected to cover a wide range of exhaust temperature, and $\mathrm{SCR}$ inlet $\mathrm{NO}_{2} / \mathrm{NO}_{x}$ ratios. The $\mathrm{DEF}$ injection flow rate commands were selected to realize different $\mathrm{SCR}$ ammonia to $\mathrm{NO}_{x}$ ratios (ANR) for each test point. Knowing the flow rate of DEF injected into the exhaust is required. Since there is not a direct measurement of DEF, an open loop DEF flow rate calibration method was developed and is described in Appendix B.

\subsubsection{Test Matrix}

The engine speed/load points, shown in Table 3.11, were selected to span an exhaust temperature range from 250 to $400^{\circ} \mathrm{C}$, which reflects typical engine operating conditions. Point 8 is selected as a baseline point, this point was run before and after each SCR test point to make sure that the engine operating condition and instrumentation are repeatable for each test. Test point 1 and 4 were repeated to verify the repeatability of the test. Lower than $250^{\circ} \mathrm{C}$ temperature points were avoided because of potential urea deposit formation in the exhaust pipe. For each engine test point, the DEF injection rate was set to realize five different $\mathrm{NH}_{3} / \mathrm{NO}_{x}$ ratios of $0.3,0.5,0.8,1.0$, and 1.2. Test plan execution facilitates acquisition of both steady-state and transient species concentrations and temperature responses suitable for both SCR model calibration and the estimator development. 
Table 3.11: Engine speed/load points for SCR tests

\begin{tabular}{|c|c|c|c|c|c|c|c|c|c|}
\hline \multirow{2}{*}{$\begin{array}{c}\text { Test } \\
\text { Point }\end{array}$} & Speed & Load & $\begin{array}{c}\text { Exhaust } \\
\text { Mass } \\
\text { Flowrate }\end{array}$ & $\begin{array}{c}\text { SCR inlet } \\
\text { Temp }\end{array}$ & $\begin{array}{c}\text { Std. SCR } \\
\text { Space } \\
\text { Velocity }\end{array}$ & $\begin{array}{c}\text { Act. } \\
\text { SCR } \\
\text { Space } \\
\text { Velocity }\end{array}$ & $\begin{array}{c}\text { SCR } \\
\text { Inlet } \\
\text { NO }\end{array}$ & $\begin{array}{c}\text { SCR } \\
\text { Inlet } \\
\text { NO }_{2}\end{array}$ & $\begin{array}{c}\mathbf{N O}_{2} / \mathbf{N O}_{\mathbf{x}} \\
\text { ratio }\end{array}$ \\
\hline 1 & 1501 & 608 & 7.40 & 399 & 23.9 & 58.9 & 462 & 295 & 0.39 \\
\hline 2 & 1715 & 500 & 7.90 & 376 & 25.3 & 60.1 & 185 & 160 & 0.46 \\
\hline 3 & 1980 & 420 & 8.38 & 353 & 26.8 & 61.5 & 115 & 187 & 0.62 \\
\hline 4 & 2097 & 342 & 8.43 & 331 & 27.0 & 59.8 & 97 & 176 & 0.64 \\
\hline 5 & 2063 & 273 & 8.99 & 301 & 28.8 & 60.6 & 78 & 220 & 0.74 \\
\hline 6 & 2153 & 194 & 9.40 & 278 & 30.1 & 60.8 & 80 & 199 & 0.71 \\
\hline 7 & 2173 & 143 & 9.76 & 252 & 31.3 & 60.2 & 110 & 120 & 0.52 \\
\hline 8 & 1600 & 475 & 7.25 & 366 & 22.9 & 53.5 & 197 & 208 & 0.51 \\
\hline
\end{tabular}

${ }^{*}$ Calculation of the space velocities is given in Appendix C.

\subsubsection{Test Procedures}

The test consists of three parts. First, the engine is run at the baseline condition to check the repeatability of the engine and instrumentation. Second, both DOC and CPF inlet and outlet gaseous emissions and engine out PM samples are taken to provide data for DOC and CPF model calibration. Third, the engine is run with varying DEF injection rates to collect SCR response data. The procedures are shown in the flow chart in Figure 3.11.

During the SCR tests, the transient responses are required. Therefore, carefully timed switching of the IMR-MS between measurement points is needed since a single IMR MS is used. The SCR outlet measurements have the highest priority. It is also important to detect any changes in the SCR inlet concentrations over a long test. The $\mathrm{NO}_{x}$ sensors can be used to give real-time indications of SCR inlet condition changes and provide a "trigger" to make a measurement upstream of the SCR. 


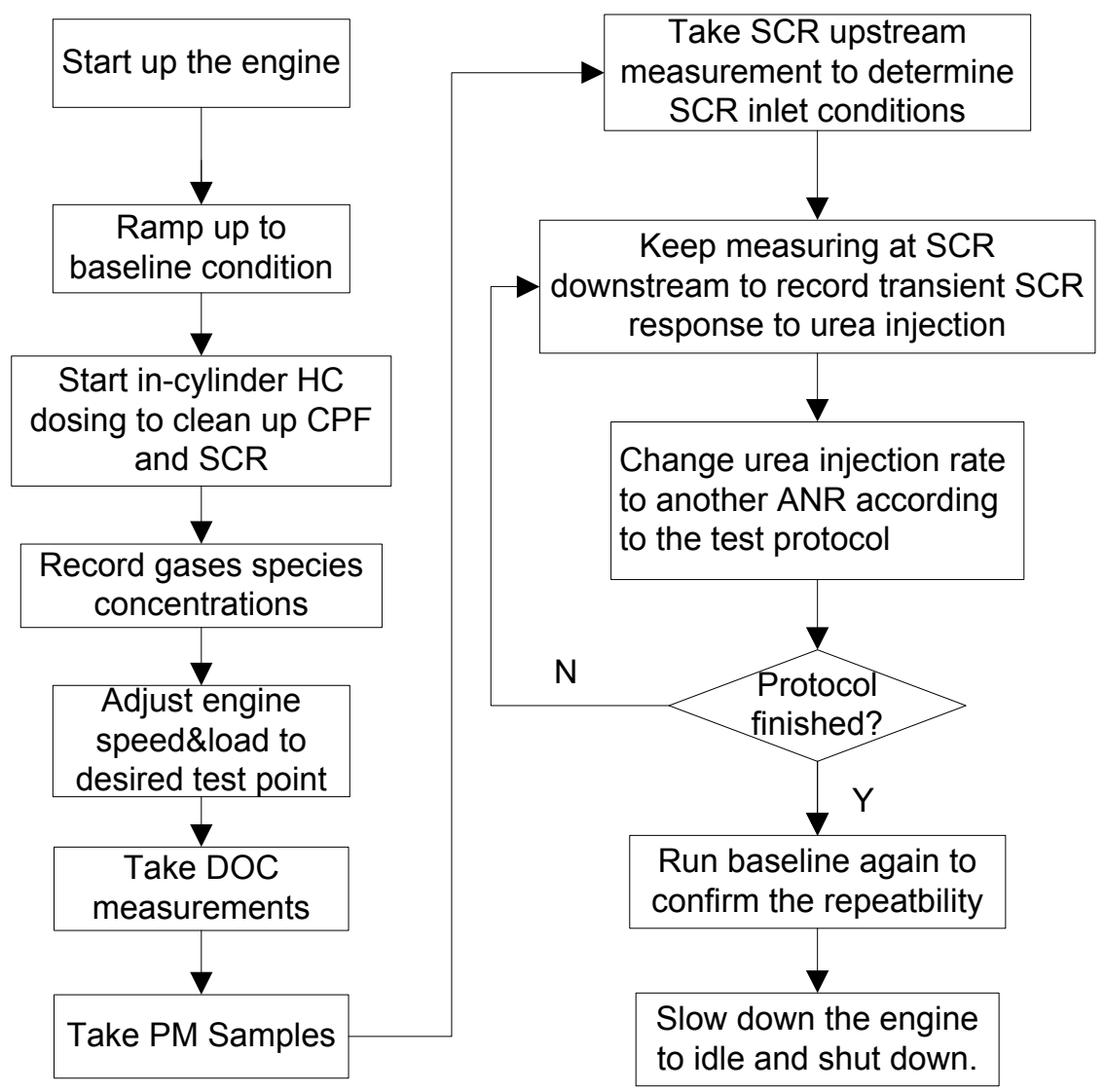

Figure 3.11: Flow chart of SCR test procedures

A step-by-step description of the test procedure illustrated in Figure 3.11 is described in the following steps:

1) Set exhaust flow to trapline. Startup engine and idle for 2 minutes. This slow ramp-up is only necessary for the first portion of a test and can be performed faster when the engine and dynamometer are warmed up.

2) Check that all sensors are providing output and are being recorded.

3) Slowly increase throttle control and dyno control to baseline condition (1600 rpm, $475 \mathrm{~N} \cdot \mathrm{m})$. 
4) Start in-cylinder HC dosing to clean up the CPF and SCR. Monitor the pressure drop across the CPF and $\mathrm{NH}_{3}$ slip at SCR outlet. Wait until the pressure drop across the CPF has stabilized and $\mathrm{NH}_{3}$ slip falls below 3 ppm.

5) Stop in-cylinder $\mathrm{HC}$ dosing, and wait until all the temperatures across the exhaust system are stabilized. Take gaseous samples to make sure the engine and instrumentation operation are repeatable.

6) Adjust speed/load to achieve the test point. Keep adjusting the intercooler control valve to adjust cooling water flow through the intercooler, until intake air temperature has stabilized at $50^{\circ} \mathrm{C}$.

7) Wait until all temperatures across the aftertreatment system are stabilized. Start recording gas species concentrations at different sampling locations for 6 minutes at each location.

8) Take three engine out PM samples, 10 minutes for each sample.

9) Record gas species concentrations at CPF outlet to determine SCR inlet conditions, and calculate the DEF injection rate needed for desired ANR.

10) Switch to SCR downstream sampling location and start DEF injection.

11) Keep measuring downstream of the SCR to record the transient SCR response to DEF injection. Once the SCR is in equilibrium state (a state that no $\mathrm{NO}_{\mathrm{x}}$ is being further reduced and there is a stabilized ammonia slip). Change DEF injection rate for another ANR based on the DEF injection schedule given in Figure 3.12.

12) Repeat step 11 until the DEF injection schedule is complete.

13) Stop DEF injection, wait until no $\mathrm{NO}_{x}$ is reduced and no $\mathrm{NH}_{3}$ slip is observed. 
14) Change engine speed/load to baseline point, and take gaseous measurements at different sampling locations to double check the repeatability of the engine and instrumentation operation.

15) Slow down the engine to idle, keep engine idling for a couple of minutes before cutting off the fuel supply.

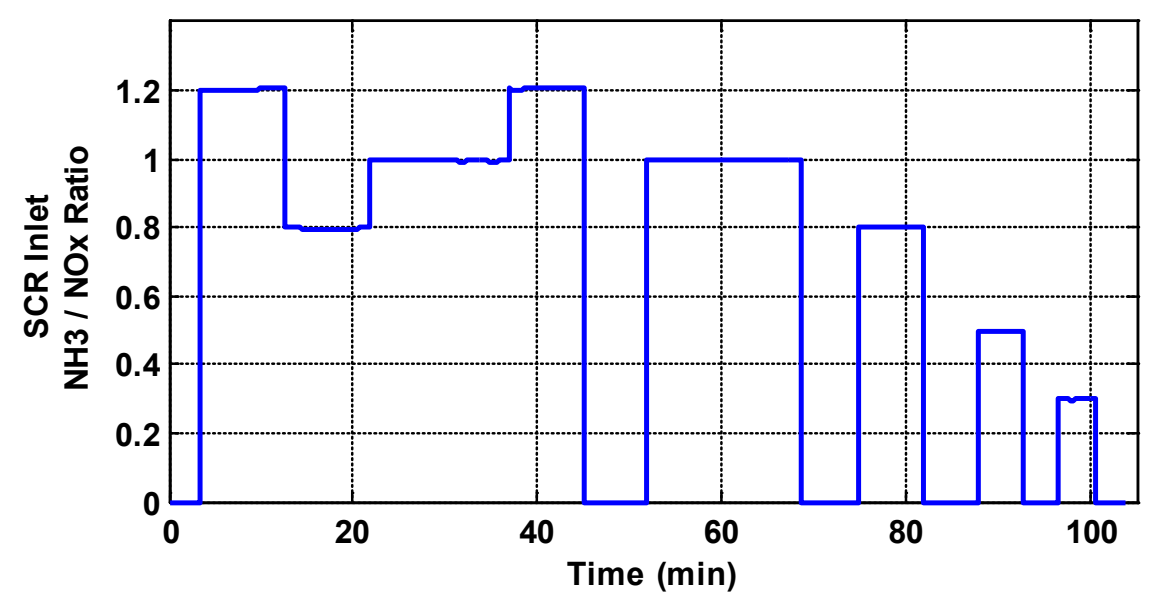

Figure 3.12: Scheduled SCR inlet ANR for steady state SCR engine test

\subsection{Transient SCR Engine Tests}

\subsubsection{Test Cell Setup for Transient Tests}

The engine test cell was developed to have the capability of running transient cycle tests. A NI analog output module ( $\mathrm{NI}$ 9263) was added into the data acquisition system to provide scheduled external voltage for controlling the dynamometer speed and engine throttle position. The switch on the front panel of the dynamometer controller and the switch newly added (shown in Figure 3.13) gives the capability of switching between the previous manual control mode and external voltage control mode. A LabView program 
was then created to drive the analog output module to generate desired voltage outputs to control both speed and fuel flow rate. The program can either take manual input values or read data points from a pre-created data file. The data file should contain desired voltage points which represent different engine speed and load conditions in a specified test cycle.

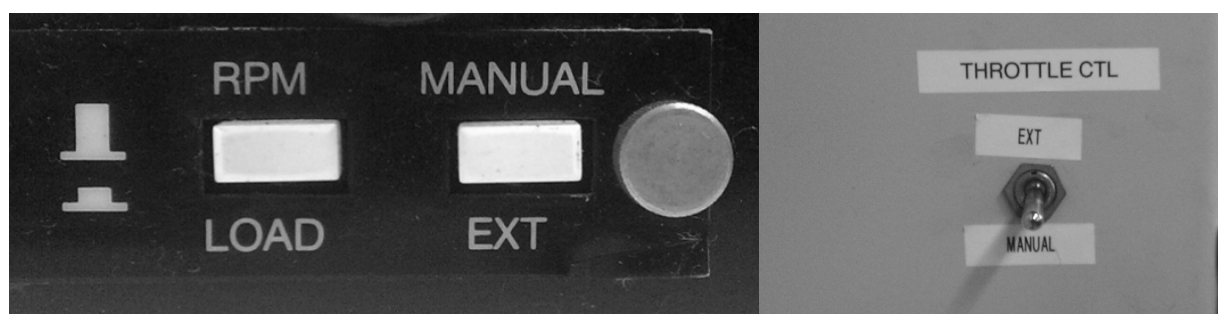

Figure 3.13: Switches used for switching between manual control mode and external voltage control mode

\subsubsection{Surrogate HD-FTP Cycle Development}

HD-FTP transient test cycle is used in the U.S. for emission testing of diesel engines for heavy-duty diesel vehicle. Four different typical driving conditions are simulated in the 20-minute long cycle which consists of a series of driving and motoring segments [127]. It is important to validate the SCR model performance under transient conditions. In order to collect SCR data under typical transient exhaust conditions, a surrogate HDFTP test cycle was developed to simulate the representative transients and make it possible to run transient tests in the engine test cell. The development procedure for the surrogate HD-FTP cycle is described.

The normalized engine conditions in terms of percent of full speed and percent of full torque as shown in plot (a) of Figure 3.14 were first scaled to the ISB engine according to the engine performance curve. Since the eddy-current dynamometer used in the test cell was not capable of running the motoring segments, the motoring points of the cycle were replaced by idle conditions. The scaled ISB engine conditions without the motoring segments are shown as plot (b) of Figure 3.14. Then the cycle was simplified by smoothing the speed and torque curves so that the cycle consisted of fewer engine 
operating conditions but still keeps the typical transient segments. The simplified cycle consisted of 38 different engine operating conditions with transition between engine operating points as shown in plot (c) of Figure 3.14.

The engine speed and torque were controlled by voltage signals generated from the $\mathrm{NI}$ 9236 analog output module. The LabView program used for testing reads a pre-defined input data file which contains the time history voltage signals corresponding to the engine speed and torque for the cycle. The analog output module generates the desired voltage signals for controlling the dynamometer speed and the fuel flow rate to the engine.

The repeatability of the cycle in terms of engine speed/torque, exhaust flow rate, exhaust temperature, and engine out $\mathrm{NO}_{\mathrm{x}}$ concentration measured by the $\mathrm{NO}_{\mathrm{x}}$ sensor were validated by comparing the measurements of multiple runs of the developed cycle as shown in Figure 3.15. The cycle was started at a specific engine operating condition to keep the SCR conditions during the cycle consistent. The engine operating conditions as well as the exhaust conditions of the cycle were repeatable except a spike in exhaust mass flow rate and engine out $\mathrm{NO}_{x}$ concentration at about 13 minutes. For a warmed up engine, it is feasible to use a single analyzer for transient testing by separately measuring inlet and outlet conditions of the catalyst during separate runs of the same cycle since the cycle is repeatable. 

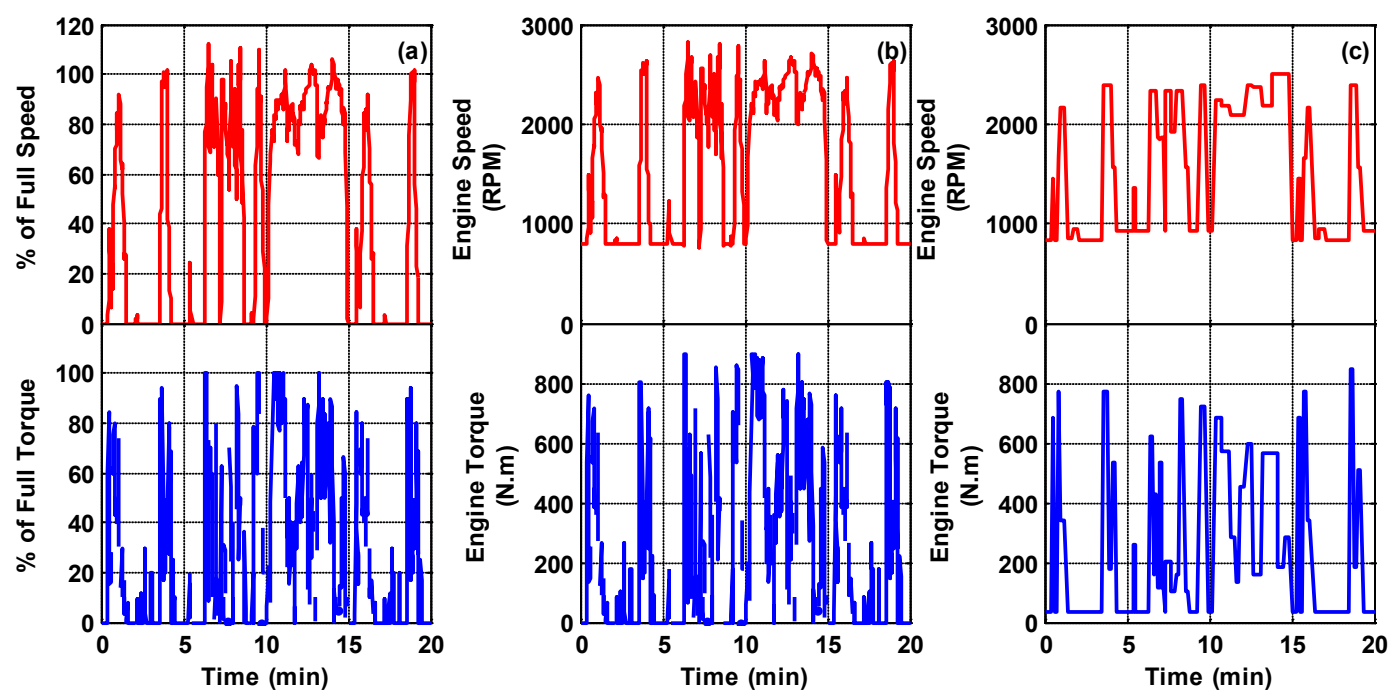

Figure 3.14: Engine conditions of the HD-FTP cycle and the developed surrogate HDFTP cycle

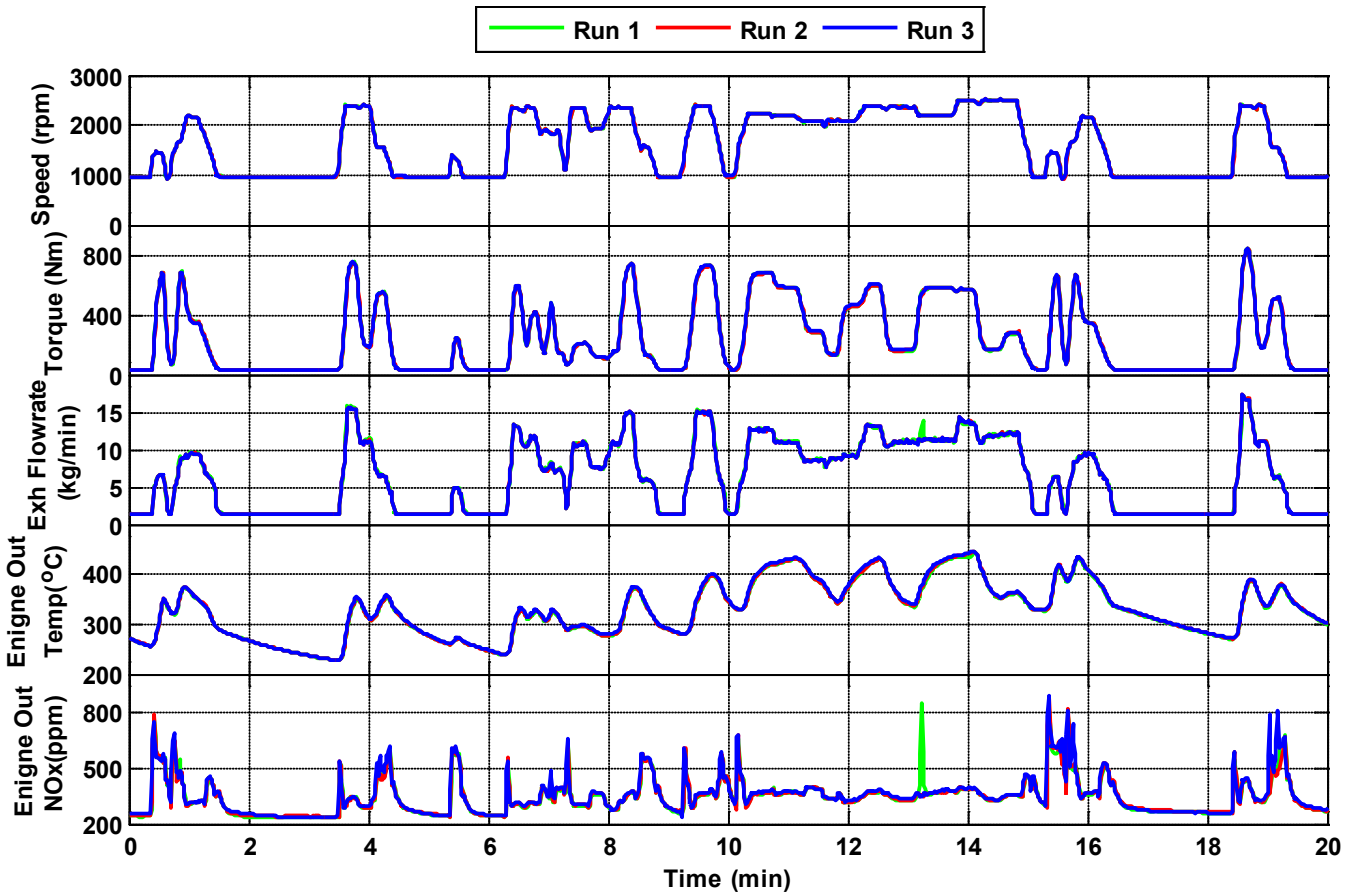

Figure 3.15: Comparison the experimental measurements for different runs of the developed surrogate HD-FTP cycle 


\subsubsection{Test Matrix}

The transient trends in SCR inlet concentrations of $\mathrm{NO}$ and $\mathrm{NO}_{2}$, SCR inlet temperature and standard SCR space velocity over the developed surrogate HD-FTP cycle are shown in Figure 3.16.

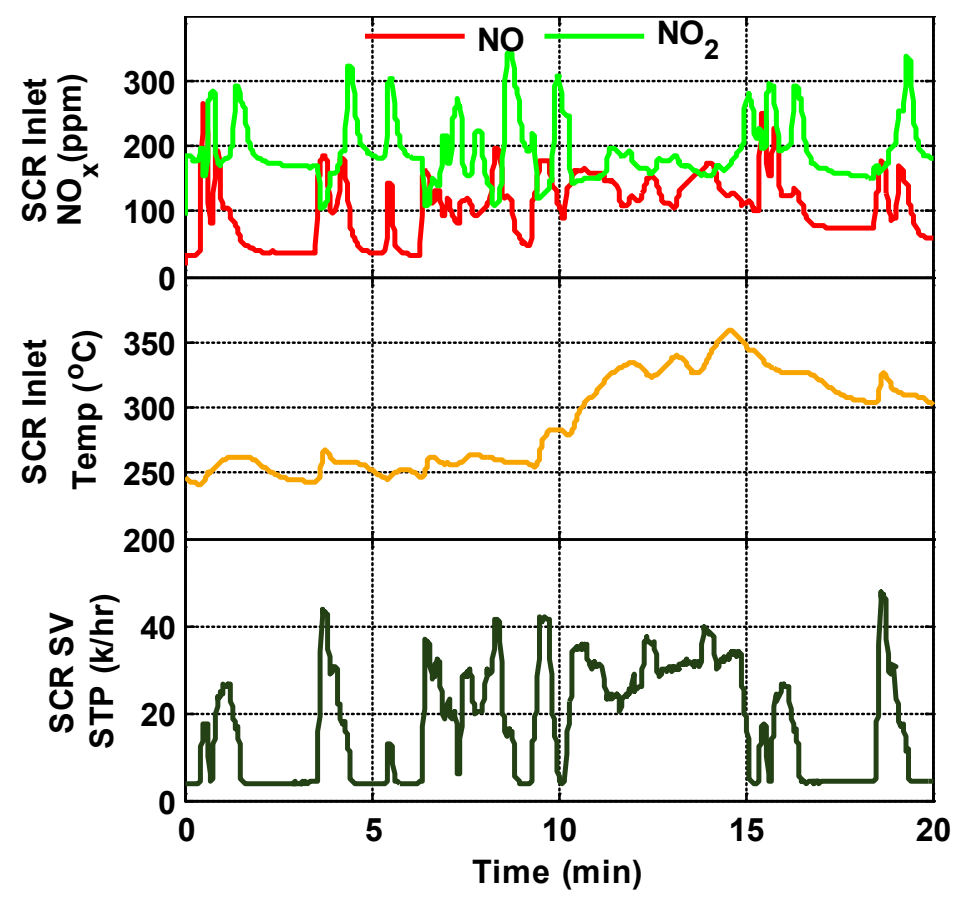

Figure 3.16: SCR inlet $\mathrm{NO}, \mathrm{NO}_{2}$ concentrations, SCR inlet temperature, and SCR standard space velocity during the surrogate HD-FTP cycle

Six transient runs with different DEF injection levels were conducted to collect transient SCR data. A transient test matrix is shown in Table 3.12. The default DEF injection level of the cycle was determined by the control strategy implanted in the engine ECM. The ECM parameter was manually overwritten to achieve $0.3,0.5,0.8,1.0$, and 1.2 times of the default overall DEF injection rate for the full cycle. Another transient test was conducted with manual control of DEF injection. The DEF injection was manually turned on and off to study both $\mathrm{NH}_{3}$ adsorption and desorption under transient conditions. The IMR-MS was used for emission measurements for the transient experiments. 
Table 3.12: Test matrix of transient SCR tests

\begin{tabular}{|c|c|}
\hline Test No. & $\begin{array}{c}\text { DEF Injection } \\
\text { Level }\end{array}$ \\
\hline 1 & Manual \\
\hline 2 & 1.2 \\
\hline 3 & 1.0 \\
\hline 4 & 0.8 \\
\hline 5 & 0.5 \\
\hline 6 & 0.3 \\
\hline
\end{tabular}

\subsubsection{Test Procedures}

A CPF active regeneration process was carried out before running each transient cycle in order to oxidize the PM mass retained in the CPF and $\mathrm{NH}_{3}$ stored in the SCR. The CPF active regeneration was carried out under the engine operating condition of 1600 rpm and $475 \mathrm{~N} \cdot \mathrm{m}$ with a target CPF inlet temperature of $550^{\circ} \mathrm{C}$ for 15 minutes or until the pressure drop across the CPF was constant. After the CPF active regeneration process, the engine was run at a steady state condition for 10 minutes as shown in subplot (a) Figure 3.17 to in order to lower the aftertreatment system temperature to $250^{\circ} \mathrm{C}$. The transient operating conditions from 10 to 20 minutes shown in Figure 3.17 was run before starting the surrogate FTP cycle. The purpose of this was to move the EGR valve in order to prevent any valve sticking problem. The following 20 minutes segment which is between 20 and 40 minutes is the surrogate HD-FTP cycle. The DEF injection was off before the 20 minute time point to start the surrogate HD-FTP cycle with zero $\mathrm{NH}_{3}$ storage on the catalyst. The engine operating condition was switched back to steady state after finishing the cycle. Subplot (b) of the Figure 3.17 shows the voltage signals used for driving the engine to perform the transient tests. 

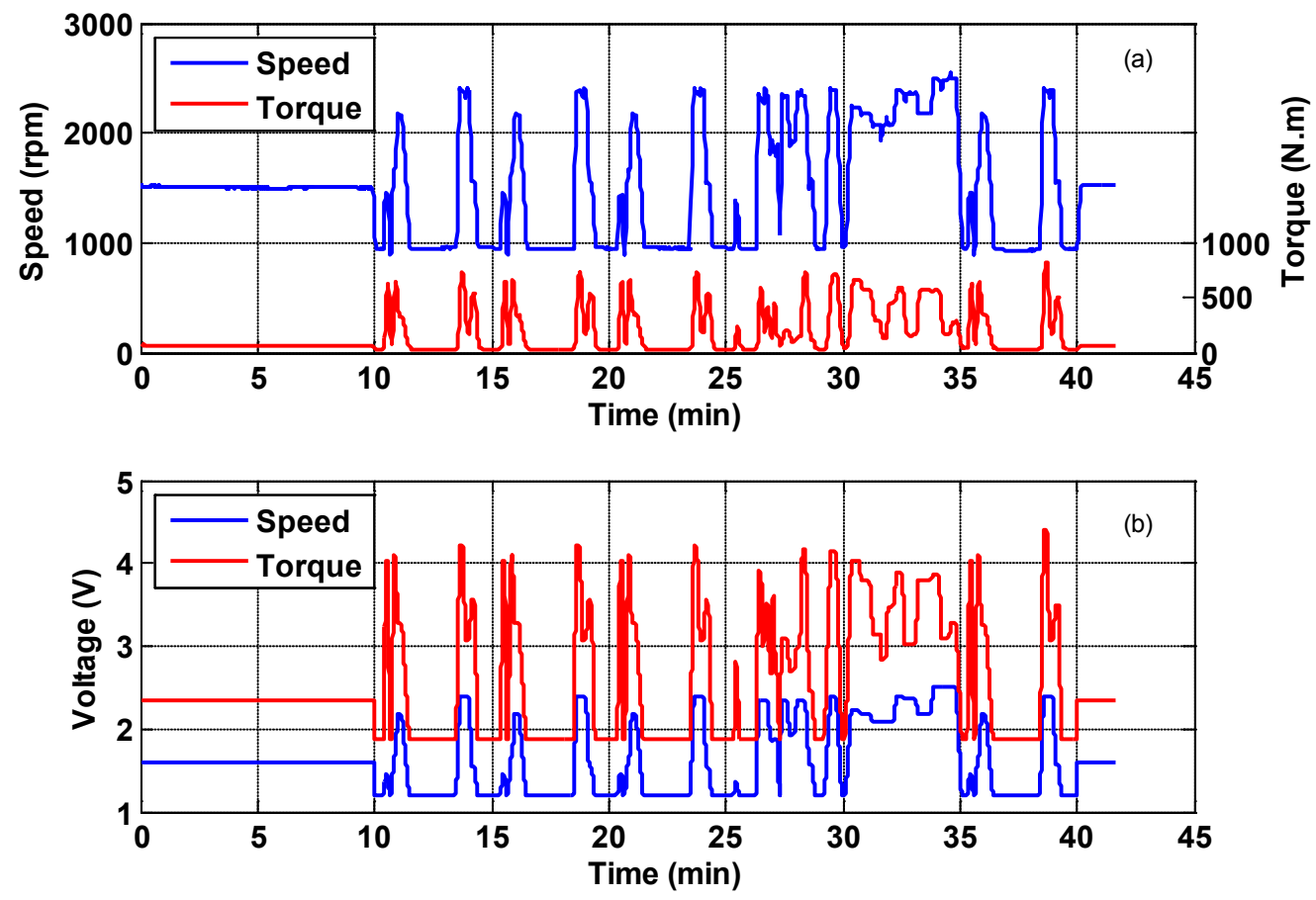

Figure 3.17: Engine conditions and control voltages for the transient test

For each of the transient tests in the test matrix shown in Table 3.12, the surrogate HDFTP cycle was run with continuous emission measurements taken from downstream of the CPF to determine the SCR inlet concentrations for the cycle. Then the emission measurement point was switched to the downstream SCR position for outlet concentration measurements during the $2^{\text {nd }}$ run of the cycle with upstream DEF injections. The intake air flow rate, pressure drops and temperatures were continuously monitored and recorded during each cycle. Gaseous concentrations of $\mathrm{NO}, \mathrm{NO}_{2}$ and $\mathrm{NH}_{3}$ were measured using the IMR- MS, and the $\mathrm{HC}, \mathrm{CO}, \mathrm{CO}_{2}$, and $\mathrm{O}_{2}$ concentrations were recorded by the AVL Pierburg five gas analyzer. The fuel flow rate measuring principle of the AVL fueling system made it unable to measure real time fuel flow rate for transient cycle. The air/fuel ratio back calculated from the exhaust and the measured intake air flow rate was used to calculate the fuel flow rates during the transient cycle. The calculation procedure is given in Appendix D. The transient experimental data were used for validating the SCR model performance. 


\subsection{Sample Line Test}

It was noticed during the steady-state SCR tests that the $\mathrm{NH}_{3}$ slip measurements from the IMR-MS were delayed because of the $\mathrm{NH}_{3}$ adsorption on the sample line wall. Using the delayed $\mathrm{NH}_{3}$ measurements in the $\mathrm{SCR}$ model calibration process without correction causes an error in the model kinetic parameters. As a result, it is important to quantify the $\mathrm{NH}_{3}$ adsorption and desorption process in the sample line and take it into consideration during calibration of the SCR model.

$\mathrm{A} \mathrm{NH}_{3}$ sensor was installed at the end of the steady-state testing phase. The sensor was located near the $\mathrm{NO}_{\mathrm{x}}$ sensor which was also downstream of the SCR. The location of the sensors was $1.5 \mathrm{~m}$ from the outlet face of the $2^{\text {nd }}$ SCR catalyst to allow the gas species to be uniformly mixed. A mixer was installed between the SCR outlet face and the sensors to improve the mixing and make sure that the $\mathrm{NH}_{3}$ sensor, $\mathrm{NO}_{\mathrm{x}}$ sensor and the emission analyzers are measuring the same exhaust gas components when measuring downstream of the SCR.

An engine test for comparison of the $\mathrm{NH}_{3}$ measurements from the IMR-MS, the $\mathrm{NH}_{3}$ sensor, and the $\mathrm{NO}_{x}$ sensor (which is cross sensitive to $\mathrm{NH}_{3}$ ) was conducted. $\mathrm{A}$ calibration of the $\mathrm{NO}_{x}$ sensor is shown in Equation 3.1. The test was performed for a SCR inlet temperature of $300{ }^{\circ} \mathrm{C}$ and a SCR space velocity of $60 \mathrm{k} \mathrm{hr}^{-1}$. The SCR inlet concentrations of $\mathrm{NO}, \mathrm{NO}_{2}$, and available $\mathrm{NH}_{3}$ formed from the $\mathrm{DEF}$ injection are given in the top plot of Figure 3.18. Similar to the steady state test procedure presented in the previous section, the gas sampling was first taken at the SCR inlet with the engine running at steady state to determine the inlet concentrations of $\mathrm{NO}$ and $\mathrm{NO}_{2}$. The DEF injection rate command for the desired SCR inlet ANR was then back calculated. Then the gas sampling was switched to downstream of the SCR to measure the outlet concentrations of $\mathrm{NO}, \mathrm{NO}_{2}$, and $\mathrm{NH}_{3}$ slip with upstream DEF injection. The SCR outlet concentrations of $\mathrm{NO}, \mathrm{NO}_{2}$ and $\mathrm{NH}_{3}$ from the IMR - MS and the sensors are given in the bottom plot of Figure 3.18. The $\mathrm{NH}_{3}$ sensor measurements were calibrated to the IMRMS as described in Appendix E. The SCR inlet ANR was set to 2.0 for this test to get close to $100 \%$ of $\mathrm{NO}_{x}$ reduction so that the measurement from the $\mathrm{NO}_{x}$ sensor was the 
$\mathrm{NH}_{3}$ concentration instead of a combination of $\mathrm{NO}_{x}$ and $\mathrm{NH}_{3}$ concentrations between 10 and 30 minutes of the test time. The $\mathrm{NO}_{x}$ sensor measurement shows different amplitude between 20 and 30 minutes probably because it has a different sensitivity to $\mathrm{NH}_{3}$ compared to the IMR-MS.

$$
\mathrm{NO}_{x}=1.21 \mathrm{NO}+0.85 \mathrm{NO}_{2}+1.05 \mathrm{NH}_{3}
$$

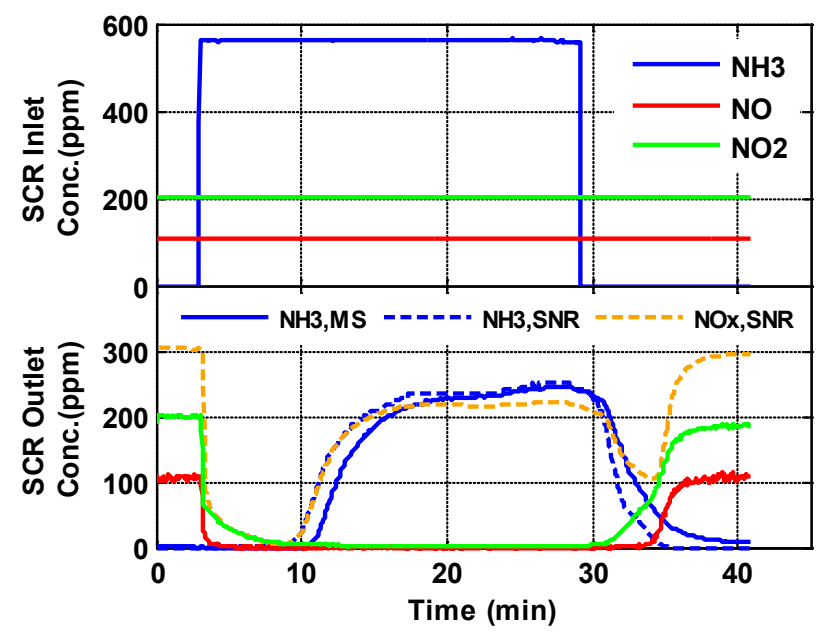

Figure 3.18: Comparison of $\mathrm{NH}_{3}$ slip measurements from the IMR-MS, the $\mathrm{NH}_{3}\left(\mathrm{NH}_{3}\right.$, $\mathrm{SNR})$ and the $\mathrm{NO}_{\mathrm{x}}$ sensors $\left(\mathrm{NO}_{\mathrm{x}}, \mathrm{SNR}\right)$

It can be observed from Figure 3.18 that the $\mathrm{NH}_{3}$ slip measurements from both sensors started just before 10 minutes. However, the IMR-MS measured $\mathrm{NH}_{3}$ slip was delayed by 2 minutes. After the DEF injection was turned off at 29 minutes, the $\mathrm{NH}_{3}$ slip measured by the $\mathrm{NH}_{3}$ sensor dropped faster than the IMR-MS. The $\mathrm{NH}_{3}$ sensor shows no slip beyond 35 minutes while the $\mathrm{NH}_{3}$ measurement from the IMR-MS was present until the end of the test at $\mathbf{4 0}$ minutes. This phenomenon was determined to be caused by the $\mathrm{NH}_{3}$ adsorption/desorption on the wall of the sample line. When exhaust gas flows through the heated sample line $\left(190^{\circ} \mathrm{C}\right)$, the $\mathrm{NH}_{3}$ gets adsorbed on the sample line wall because of its adsorption properties. $\mathrm{No}^{\mathrm{NH}_{3}}$ can be detected by the analyzer until the $\mathrm{NH}_{3}$ breaks through the storage capacity of the sample line. After DEF injection was turned off, the $\mathrm{NH}_{3}$ slip gradually disappears from the exhaust stream going through the 
sample line. The $\mathrm{NH}_{3}$ previously stored in the sample line starts to be slowly desorbed until no $\mathrm{NH}_{3}$ is stored in the sample line.

The $\mathrm{NH}_{3}$ adsorption on the sample line wall needs to be quantified and taken into consideration when calibrating the SCR model to the engine IMR-MS experimental data. In order to characterize this phenomenon and eliminate the effect of $\mathrm{NH}_{3}$ measurement delay on the SCR kinetics study, another sample line test was conducted by measuring the $\mathrm{NH}_{3}$ calibration gas directly from a gas cylinder with known $\mathrm{NH}_{3}$ concentration of $103.8 \mathrm{ppm}$ using the IMR-MS with a sampling flow rate of $4 \mathrm{~L} / \mathrm{min}$. The calibration gas pressure was regulated to be atmospheric pressure using a 2-stage pressure regulator. The sample line was used to connect the gas cylinder and the analyzer. A valve was installed at the inlet of the sample line to switch sampling flow between the room air and the cylinder. The test was conducted to determine the IMR-MS analyzer response to a step input of a flow with known $\mathrm{NH}_{3}$ concentration in sample line. The data were used to develop a sample line model which simulates the $\mathrm{NH}_{3}$ adsorption and desorption processes.

\section{7 $\quad \mathrm{NH}_{3}$ Maldistribution Tests}

The test cell was initially setup with emissions sampling location right after each of the SCR bricks. The preliminary test results with emission measurements taken close to the SCR outlet face showed unbalanced $\mathrm{NH}_{3}+\mathrm{NO}_{x}$ concentrations across the SCR during upstream DEF injection. The reason was determined to be that the injected DEF is not well mixed with the exhaust before going into the SCR, causing nonuniformity of $\mathrm{NO}_{\mathrm{x}}$ and $\mathrm{NH}_{3}$ after the SCR. The design of the sampling probe was not able to provide average concentration measurements across the diameter of the catalyst. After moving down from the SCR outlet sampling location further from the SCR outlet face and installing an exhaust mixer, the $\mathrm{NH}_{3}+\mathrm{NO}_{\mathrm{x}}$ concentrations were balanced before and after the SCR. The $\mathrm{NH}_{3}$ maldistribution engine tests were aimed at determining the SCR inlet $\mathrm{NH}_{3}$ and ANR distribution profiles with different mixer setups. The test setup, procedures are introduced in this section. 
The SCR inlet $\mathrm{NH}_{3}$ concentration cannot be directly measured using the emission analyzers because the urea injected into the exhaust flow may crystallize in the sample line and damage the analyzer. As a result, the SCR inlet $\mathrm{NH}_{3}$ maldistribution was determined by comparing the $\mathrm{NO}_{\mathrm{x}}$ distribution at the outlet face of the 1st SCR brick with and without the SCR upstream DEF injection. The distribution of the $\mathrm{NO}_{\mathrm{x}}$ reduced across the SCR with DEF injections was considered to be the $\mathrm{NH}_{3}$ distribution of the SCR inlet face. In order to determine the distribution profiles, gas sampling was taken from different radial positions downstream of the 1 st SCR brick using $1 / 4$ inch stainless steel sampling probes shown in Figure 3.19. The measurement positions at the 1st SCR outlet face are shown in Figure 3.20. Sampling probes were extended into seven different radial positions from $A, B, C$, and D positions on the SCR can. A total of 28 radial positions were sampled. The average of $A 4, B 4, C 4$, and D4 measurements was considered as the center measurement. All the other 24 measurement points are located in the center of 24 equal inlet areas.

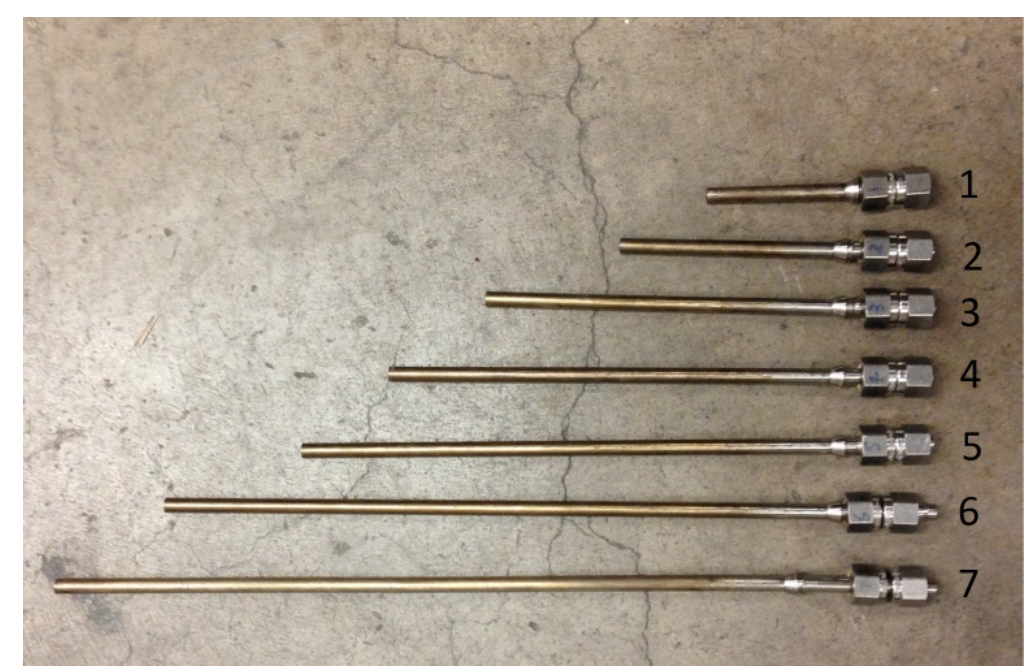

Figure 3.19: Sampling probes for different radial measurement locations 


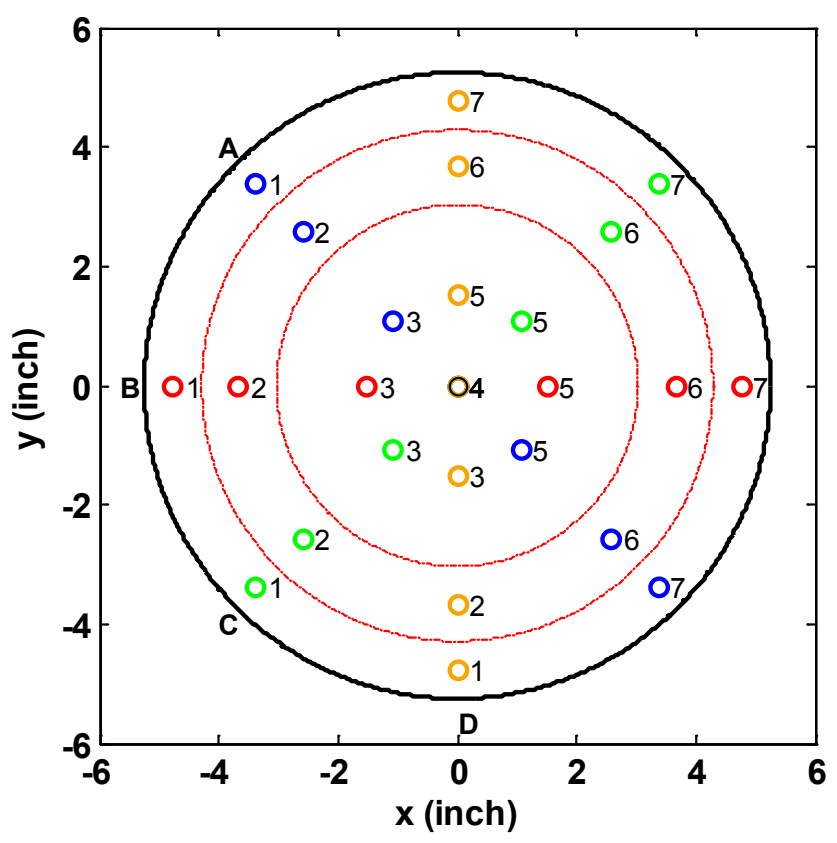

Figure 3.20: Gaseous sampling positions at the outlet face of the 1st SCR brick

The gaseous measurements of $\mathrm{NO}$ and $\mathrm{NO}_{2}$ at each of the positions were measured with and without the DEF injections. During the maldistribution test, the $\mathrm{NO}$ and $\mathrm{NO}_{2}$ concentrations at each measurement location were first measured after the engine and aftertreatment system conditions reached steady state. Then, DEF injection was initiated upstream of the SCR. The $\mathrm{NO}$ and $\mathrm{NO}_{2}$ concentrations at each measurement location were measured again after the SCR reached steady state. The IMR-MS was used for the gaseous concentration measurements. The SCR $\mathrm{NH}_{3}$ concentration at each measurement location was determined by calculating the differences in $\mathrm{NO}_{\mathrm{x}}$ concentrations with and without the upstream DEF injection.

The test matrix for the $\mathrm{NH}_{3}$ maldistribution test is shown in Table 3.13. A total of six tests were conducted. The two exhaust mass flow rates tested represented low and high flow rates of the engine along with two SCR inlet temperatures of 300 and $350^{\circ} \mathrm{C}$. The SCR inlet temperature was controlled and maintained by using the $25 \mathrm{~kW}$ exhaust heater. Lower temperatures were avoided because of the potential formation of solid ammonium nitrate affecting the $\mathrm{NH}_{3}$ balance. Higher temperatures were also not tested because of the increasing $\mathrm{NH}_{3}$ oxidation rate. Tests number 1, 2 and 3 were performed with the 
original mixer setup. Tests number 4, 5 and 6 were performed with a swirl mixer installed into the SCR inlet cone.

Table 3.13: Test matrix for the $\mathrm{NH}_{3}$ maldistribution test

\begin{tabular}{|c|c|c|c|c|c|c|c|}
\hline $\begin{array}{l}\text { Test } \\
\text { No. }\end{array}$ & $\begin{array}{c}\text { Engine } \\
\text { Speed } \\
(\mathrm{rpm})\end{array}$ & $\begin{array}{c}\text { Engine } \\
\text { Torque } \\
(\mathrm{N} \cdot \mathrm{m})\end{array}$ & $\begin{array}{c}\text { Exh Mass } \\
\text { Flow } \\
\text { Rate } \\
\text { (kg/min) }\end{array}$ & $\begin{array}{c}\text { SCR } \\
\text { Inlet } \\
\text { Temp. } \\
\left({ }^{\circ} \mathrm{C}\right)\end{array}$ & $\begin{array}{c}\text { SCR } \\
\text { Inlet NO } \\
\text { (ppm) }\end{array}$ & $\begin{array}{c}\text { SCR } \\
\text { Inlet NO } \\
(p p m)\end{array}$ & $\begin{array}{c}\text { SCR Inlet } \\
\text { Average } \\
\text { ANR }\end{array}$ \\
\hline 1 & 1450 & 203 & 4.23 & 350 & 170 & 192 & 0.30 \\
\hline 2 & 2500 & 245 & 9.89 & 350 & 150 & 131 & 0.30 \\
\hline 3 & 2500 & 245 & 9.89 & 350 & 150 & 131 & 0.65 \\
\hline $4^{*}$ & 1450 & 200 & 4.20 & 300 & 229 & 120 & 0.30 \\
\hline $5^{*}$ & 1450 & 200 & 4.20 & 350 & 171 & 170 & 0.30 \\
\hline $6^{*}$ & 2500 & 240 & 10.0 & 350 & 146 & 126 & 0.30 \\
\hline
\end{tabular}

* Test No. 4, 5 and 6 were performed with an additional swirl mixer installed into the SCR inlet cone.

\subsection{Flow Reactor Tests}

\subsubsection{Flow Reactor Setup}

The flow reactor SCR experiments were conducted in an automated flow reactor at Oak Ridge National Laboratory (ORNL). "The flow reactor uses synthetic exhaust gas mixtures to probe specific functions and properties of catalytic materials. Gas compositions were mixed from compressed gas bottles using mass flow controllers. Water vapor was introduced through a high performance liquid chromatography (HPLC) pump and a custom capillary injection vaporizer system. Two four-way pneumatically actuated switching valves directed the flow of $\mathrm{NH}_{3}$ and $\mathrm{NO}_{x}$ either to the reactor or to an exhaust line, creating approximately stepwise changes in inlet gas composition. All of the stainless steel reactor gas lines were heated to roughly $200^{\circ} \mathrm{C}$ to prevent water condensation, adsorption of $\mathrm{NH}_{3}$, and formation of $\mathrm{NH}_{4} \mathrm{NO}_{3}$. The catalyst core sample was loaded in a $2.5 \mathrm{~cm}$ diameter quartz tube with custom stainless steel end caps and placed in a tube furnace to control the catalyst temperature. Type $\mathrm{K}$ thermocouples $(0.5$ $\mathrm{mm}$ diameter) were deployed $5 \mathrm{~mm}$ upstream, $5 \mathrm{~mm}$ downstream, and at the midpoint inside the catalyst core sample to monitor catalyst temperatures. Gas concentrations upstream and downstream of the catalyst sample were measured with an MKS Multigas 
2030HS Fourier Transform Infrared (FTIR) spectrometer. A custom LabVIEW interface provided automated data acquisition and system control." [128]

\subsubsection{Catalyst Sample}

The core sample used in the reactor tests came from another Cu-zeolite SCR catalyst used in the 2010 Cummins ISB engine. The core SCR sample was cut from the front face of the full size catalyst monolith. The details of the catalyst sample are given in Table 3.14. The core sample was de-greened in a laboratory furnace at $700^{\circ} \mathrm{C}$ for four hours under a flow of $20 \% \mathrm{O}_{2}$ and $4.5 \% \mathrm{H}_{2} \mathrm{O}$ in a balance of $\mathrm{N}_{2}$ before reactor experiments to stabilize the catalyst performance.

Table 3.14 Details of the core SCR sample

\begin{tabular}{|l|c|}
\hline Catalyst material & Cu-zeolite \\
\hline Core diameter $(\mathrm{cm})$ & 2.0 \\
\hline Core length $(\mathrm{cm})$ & 3.0 \\
\hline Core volume* $\left(\mathrm{cm}^{3}\right)$ & 9.42 \\
\hline Number of channels & 162 \\
\hline De-green condition & $\begin{array}{r}700^{\circ} \mathrm{C} \text { for } 4 \text { hours, under } 20 \% \text { of } \mathrm{O}_{2}, \\
4.5 \% \text { of } \mathrm{H}_{2} \mathrm{O}, \text { balanced with } \mathrm{N}_{2}\end{array}$ \\
\hline
\end{tabular}

* Used for space velocity and $\mathrm{NH}_{3}$ storage capacity calculations

\subsubsection{Test Protocol}

The reactor experiment protocol was developed to measure the key catalyst properties that control SCR NO $\mathrm{N}_{x}$ conversion performance. The isothermal protocol consisted of a series of step changes in inlet gas composition designed to measure $\mathrm{NH}_{3}$ inventories and reaction rates under both transient and steady state SCR operating conditions. The changes in inlet composition for a single run of the isothermal protocol are shown in Figure 3.21, while the detailed composition for each step in the protocol is shown in Table 3.15. The exhaust flow contains $5 \%$ of $\mathrm{H}_{2} \mathrm{O}$ and $5 \%$ of $\mathrm{CO}_{2}$ for all protocol steps to ensure relevance to exhaust conditions. Each step in the protocol was allowed to run 
until the outlet gas concentrations reached a steady state. The protocol was repeated over a range of temperatures and space velocities.

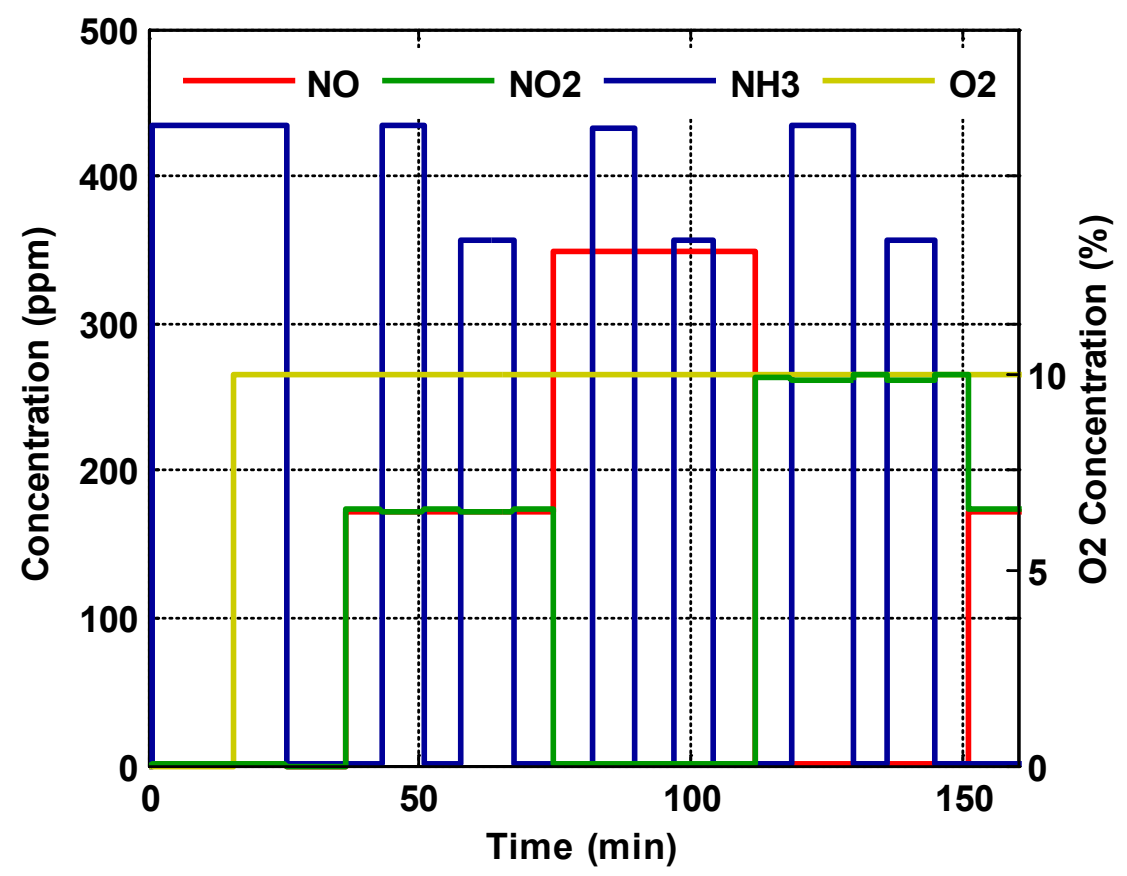

Figure 3.21: Test protocol showing the inlet gas concentrations for reactor testing 
Table 3.15: Gas species concentrations for each test step of the reactor test protocol

\begin{tabular}{|c|c|c|c|c|c|c|c|}
\hline Step & Description & $\begin{array}{c}\text { NO } \\
(\mathrm{ppm})\end{array}$ & $\begin{array}{c}\mathrm{NO}_{2} \\
(\mathrm{ppm})\end{array}$ & $\begin{array}{c}\mathrm{NH}_{3} \\
\text { (ppm) }\end{array}$ & $\begin{array}{l}\mathrm{O}_{2} \\
(\%)\end{array}$ & $\begin{array}{l}\mathrm{H}_{2} \mathrm{O} \\
(\%)\end{array}$ & $\begin{array}{l}\mathrm{CO}_{2} \\
(\%)\end{array}$ \\
\hline 1 & stabilize & 0 & 0 & 0 & 0 & 5 & 5 \\
\hline 2 & $\mathrm{NH}_{3}$ adsorption & 0 & 0 & 420 & 0 & 5 & 5 \\
\hline 3 & $\mathrm{NH}_{3}$ oxidation & 0 & 0 & 420 & 10 & 5 & 5 \\
\hline 4 & $\mathrm{NH}_{3}$ desorption & 0 & 0 & 0 & 10 & 5 & 5 \\
\hline 5 & $\begin{array}{l}\mathrm{NH}_{3} \text { inventory: } \\
\mathrm{NO}_{2} / \mathrm{NO}_{\mathrm{x}}=0.5\end{array}$ & 175 & 175 & 0 & 10 & 5 & 5 \\
\hline 6 & $\begin{array}{c}\text { SCR: } \mathrm{NH}_{3} / \mathrm{NO}_{\mathrm{x}}=1.2 ; \\
\mathrm{NO}_{2} / \mathrm{NO}_{\mathrm{x}}=0.5\end{array}$ & 175 & 175 & 420 & 10 & 5 & 5 \\
\hline 7 & $\begin{array}{c}\mathrm{NH}_{3} \text { inventory: } \\
\mathrm{NO}_{2} / \mathrm{NO}_{\mathrm{x}}=0.5\end{array}$ & 175 & 175 & 0 & 10 & 5 & 5 \\
\hline 8 & $\begin{array}{c}\text { SCR: } \\
\mathrm{NH}_{3} / \mathrm{NO}_{\mathrm{x}}=1.0 ; \\
\mathrm{NO}_{2} / \mathrm{NO}_{\mathrm{x}}=0.5\end{array}$ & 175 & 175 & 350 & 10 & 5 & 5 \\
\hline 9 & $\begin{array}{l}\mathrm{NH}_{3} \text { inventory: } \\
\mathrm{NO}_{2} / \mathrm{NO}_{\mathrm{x}}=0.5\end{array}$ & 175 & 175 & 0 & 10 & 5 & 5 \\
\hline 10 & $\begin{array}{c}\mathrm{NO} \text { oxid: } \\
\mathrm{NO}_{2} / \mathrm{NO}_{\mathrm{x}}=0.0\end{array}$ & 350 & 0 & 0 & 10 & 5 & 5 \\
\hline 11 & $\begin{array}{c}\text { SCR: } \\
\mathrm{NH}_{3} / \mathrm{NO}_{\mathrm{x}}=1.2 ; \\
\mathrm{NO}_{2} / \mathrm{NO}_{\mathrm{x}}=0.0\end{array}$ & 350 & 0 & 420 & 10 & 5 & 5 \\
\hline 12 & $\begin{array}{l}\mathrm{NH}_{3} \text { inventory: } \\
\mathrm{NO}_{2} / \mathrm{NO}_{\mathrm{x}}=0.0\end{array}$ & 350 & 0 & 0 & 10 & 5 & 5 \\
\hline 13 & $\begin{array}{c}\text { SCR: } \\
\mathrm{NH}_{3} / \mathrm{NO}_{\mathrm{x}}=1.0 \\
\mathrm{NO}_{2} / \mathrm{NO}_{\mathrm{x}}=0.0\end{array}$ & 350 & 0 & 350 & 10 & 5 & 5 \\
\hline 14 & $\begin{array}{l}\mathrm{NH}_{3} \text { inventory: } \\
\mathrm{NO}_{2} / \mathrm{NO}_{\mathrm{x}}=0.0\end{array}$ & 350 & 0 & 0 & 10 & 5 & 5 \\
\hline 15 & $\begin{array}{c}\mathrm{NO}_{2} \text { decomposition: } \\
\mathrm{NO}_{2} / \mathrm{NO}_{\mathrm{x}}=1.0\end{array}$ & 0 & 263 & 0 & 10 & 5 & 5 \\
\hline 16 & $\begin{array}{c}\text { SCR: } \\
\mathrm{NH}_{3} / \mathrm{NO}_{\mathrm{x}}=1.60 \\
\mathrm{NO}_{2} / \mathrm{NO}_{\mathrm{x}}=1.0\end{array}$ & 0 & 263 & 420 & 10 & 5 & 5 \\
\hline 17 & $\begin{array}{l}\mathrm{NH}_{3} \text { inventory: } \\
\mathrm{NO}_{2} / \mathrm{NO}_{\mathrm{x}}=1.0\end{array}$ & 0 & 263 & 0 & 10 & 5 & 5 \\
\hline 18 & $\begin{array}{c}\text { SCR: } \\
\mathrm{NH}_{3} / \mathrm{NO}_{\mathrm{x}}=1.33 ; \\
\mathrm{NO}_{2} / \mathrm{NO}_{\mathrm{x}}=1.0\end{array}$ & 0 & 263 & 350 & 10 & 5 & 5 \\
\hline 19 & $\begin{array}{l}\mathrm{NH}_{3} \text { inventory: } \\
\mathrm{NO}_{2} / \mathrm{NO}_{\mathrm{x}}=1.0\end{array}$ & 0 & 263 & 0 & 10 & 5 & 5 \\
\hline 20 & $\begin{array}{c}\text { clean: } \\
\mathrm{NO}_{2} / \mathrm{NO}_{\mathrm{x}}=0.5\end{array}$ & 175 & 175 & 0 & 10 & 5 & 5 \\
\hline
\end{tabular}


The following description of the test protocol is quoted from reference [128]. "The gas composition changes within the protocol were structured in an attempt to isolate key processes that occur over the SCR catalyst so that model parameter estimation could be performed on individual reactions or reaction subsets rather than the full SCR reaction mechanism. The protocol can be roughly divided into four sections. Step 1 of the protocol was used to stabilize the SCR conditions. The following section of the protocol (steps 2-5) focused on $\mathrm{NH}_{3}$ adsorption, desorption, and oxidation in the absence of $\mathrm{NO}_{\mathrm{x}}$. The remaining three sections measured $\mathrm{NH}_{3}$ inventories and reaction rates under three different SCR regimes, as commonly defined by the $\mathrm{NO}_{2} / \mathrm{NO}_{x}$ ratio: fast SCR $\left(\mathrm{NO}_{2} / \mathrm{NO}_{x}\right.$ $=0.5$, steps 5-9), standard or NO SCR $\left(\mathrm{NO}_{2} / \mathrm{NO}_{\mathrm{x}}=0.0\right.$, steps 10-14), and slow or $\mathrm{NO}_{2}$ SCR $\left(\mathrm{NO}_{2} / \mathrm{NO}_{x}=1.0\right.$, steps 15-19). The $\mathrm{NH}_{3}$ storage section of the protocol began in step 2 with $\mathrm{NH}_{3}$ uptake measured in the absence of $\mathrm{O}_{2}$ and $\mathrm{NO}_{x}$. This step yielded a measurement of the total $\mathrm{NH}_{3}$ storage capacity while avoiding the complications introduced by $\mathrm{NH}_{3}$ oxidation. The rate of $\mathrm{NH}_{3}$ oxidation was measured in step 3 after $\mathrm{O}_{2}$ was turned on. Step 4 measured the rate of $\mathrm{NH}_{3}$ desorption from the catalyst surface after $\mathrm{NH}_{3}$ was removed from the feed gas. Finally, in step 5, any remaining $\mathrm{NH}_{3}$ on the catalyst surface was cleaned off with a 1:1 mixture of $\mathrm{NO}$ and $\mathrm{NO}_{2}$. Integrating the $\mathrm{NH}_{3}$ desorbed in step 4 and the $\mathrm{NO}_{x}$ reduced by stored $\mathrm{NH}_{3}$ in step 5 yielded a measure of the $\mathrm{NH}_{3}$ storage under saturation conditions in the presence of $\mathrm{O}_{2}$. The three SCR sections of the protocol all followed the same pattern. As detailed in Table 3.15, the feed gas included $\mathrm{O}_{2}$ and $\mathrm{NO}_{x}$ in all of the SCR steps. The first step in each SCR section stabilized the flow of $\mathrm{NO}_{x}$ at the desired $\mathrm{NO}_{2} / \mathrm{NO}_{x}$ ratio. These steps also enabled measurement of the forward and reverse rates of the $\mathrm{NO}$ oxidation reaction (the reverse reaction is also referred to as $\mathrm{NO}_{2}$ decomposition below). Once the $\mathrm{NO}_{\mathrm{x}}$ concentrations stabilized, $420 \mathrm{ppm} \mathrm{NH}$ was turned on, corresponding to $20 \%$ excess $\mathrm{NH}_{3}$ relative to the amount needed to convert all of the $\mathrm{NO}_{x}$ to $\mathrm{N}_{2}$. After the SCR reactions came to steady state, the $\mathrm{NH}_{3}$ was turned off while $\mathrm{NO}_{x}$ continued to flow. Integrating the $\mathrm{NO}_{x}$ reduced by the $\mathrm{NH}_{3}$ stored on the catalyst during this step yielded a measure of the $\mathrm{NH}_{3}$ inventory under SCR conditions with excess $\mathrm{NH}_{3}$ in the feed. Once the catalyst surface had been cleaned, $\mathrm{NH}_{3}$ was restarted at a concentration of $350 \mathrm{ppm}$, corresponding to exactly the amount of $\mathrm{NH}_{3}$ needed to convert all of the incoming $\mathrm{NO}_{x}$ to $\mathrm{N}_{2}$. Once again, the SCR reaction was allowed to come to a steady state, and then the $\mathrm{NH}_{3}$ was shut off 
while $\mathrm{NO}_{x}$ continued to flow. Integrating the $\mathrm{NO}_{x}$ reduced by the stored $\mathrm{NH}_{3}$ allowed measurement of the $\mathrm{NH}_{3}$ inventory under stoichiometric SCR conditions."

\subsection{4 $\mathrm{NH}_{3}$ TPD Test}

The following description of the temperature programmed desorption (TPD) experiments is quoted from reference [128]. "To further investigate the effects of temperature on $\mathrm{NH}_{3}$ storage capacity and stability, TPD experiments were also conducted. Prior to the TPD experiment, the catalyst surface was cleaned by holding it at $500^{\circ} \mathrm{C}$ under $10 \% \mathrm{O}_{2}, 5 \%$ $\mathrm{H}_{2} \mathrm{O}$, and $5 \% \mathrm{CO}_{2}$ for $30 \mathrm{~min}$. The sample was then cooled to $200{ }^{\circ} \mathrm{C}$ under the same gas mixture and the temperature was stabilized before $\mathrm{O}_{2}$ was shut off. The test protocol for the $\mathrm{NH}_{3}$ TPD experiments is shown in Figure 3.22. The inlet gas included $5 \% \mathrm{H}_{2} \mathrm{O}$ and $5 \% \mathrm{CO}_{2}$ during all steps of the experiment. The experiment began with an $\mathrm{NH}_{3}$

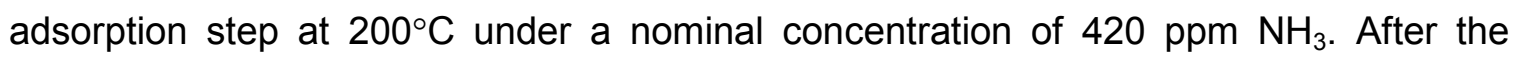
catalyst was saturated with $\mathrm{NH}_{3}$ (as evidenced by a steady state outlet concentration equal to the inlet concentration), the flow of $\mathrm{NH}_{3}$ was shut off and the catalyst was held at $200{ }^{\circ} \mathrm{C}$ while $\mathrm{NH}_{3}$ isothermally desorbed. When the rate of desorption had slowed to the point that the outlet $\mathrm{NH}_{3}$ concentration dropped below $5 \mathrm{ppm}$, the catalyst temperature was increased to $500^{\circ} \mathrm{C}$ at a rate of $5^{\circ} \mathrm{C} \mathrm{min}^{-1}$. This temperature was sufficiently high to remove all $\mathrm{NH}_{3}$ from the catalyst surface. The TPD experiments were used in validating the $\mathrm{NH}_{3}$ adsorption/desorption rates in the model."

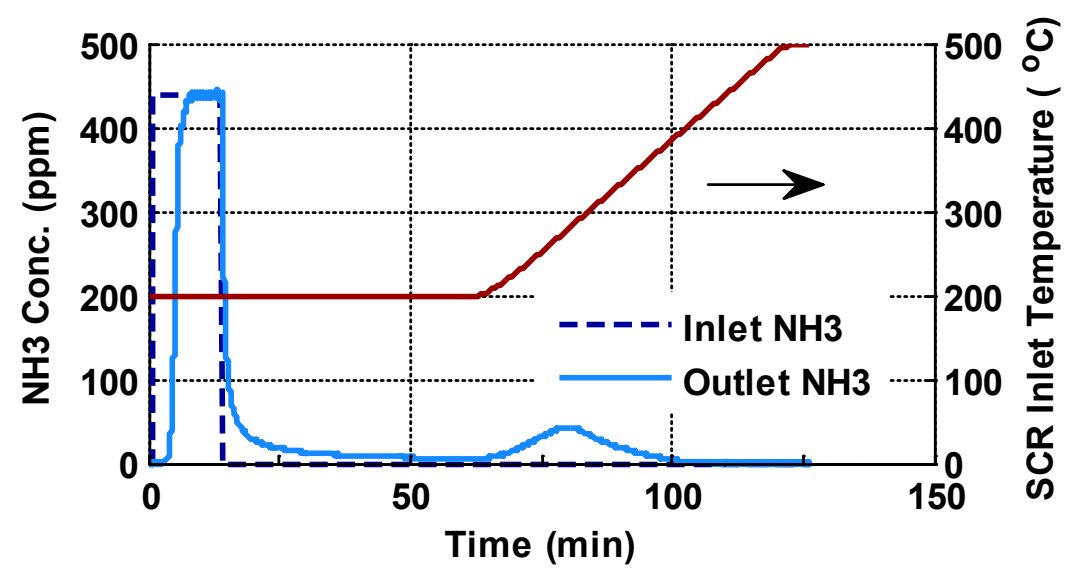

Figure 3.22: Test protocol for $\mathrm{NH}_{3}$ TPD experiments 


\subsubsection{Test Matrix}

The reactor tests were conducted over a broad range of temperatures from 200 to 425 ${ }^{\circ} \mathrm{C}$ and standard space velocities of $60,000,90,000$, and 120,000 $\mathrm{hr}^{-1}$. The standard SCR space velocity was calculated using $0^{\circ} \mathrm{C}$ and 1 atm pressure as a reference condition. The space velocity calculation is shown in Equation 3.2.

$$
S V=\frac{\text { Std Volumetric Flow Rate }}{\text { Catalyst Volume }}
$$

The reactor test matrix is given in Table 3.16. For 60,000 and $120,000 \mathrm{hr}^{-1}$, the isothermal experiment protocol was repeated at intervals of $50^{\circ} \mathrm{C}$ from 200 to $400^{\circ} \mathrm{C}$. At $90,000 \mathrm{hr}^{-1}$, the protocol was repeated at $25^{\circ} \mathrm{C}$ intervals between 200 and $425^{\circ} \mathrm{C} . \mathrm{NH}_{3}$ TPD tests were conducted for each space velocity tested. The span of the broad temperature range allowed identification of the effect of the temperature on kinetic parameters as well as determination of pre-exponential factors and activation energies for each reaction included in the model. Different space velocity tests facilitated validation of the mass transfer modeling included in the SCR model. $\mathrm{NH}_{3}$ TPD experiments provided opportunities for validating the $\mathrm{NH}_{3}$ storage and adsorption/desorption parameters.

Table 3.16: Test Matrix for reactor testing

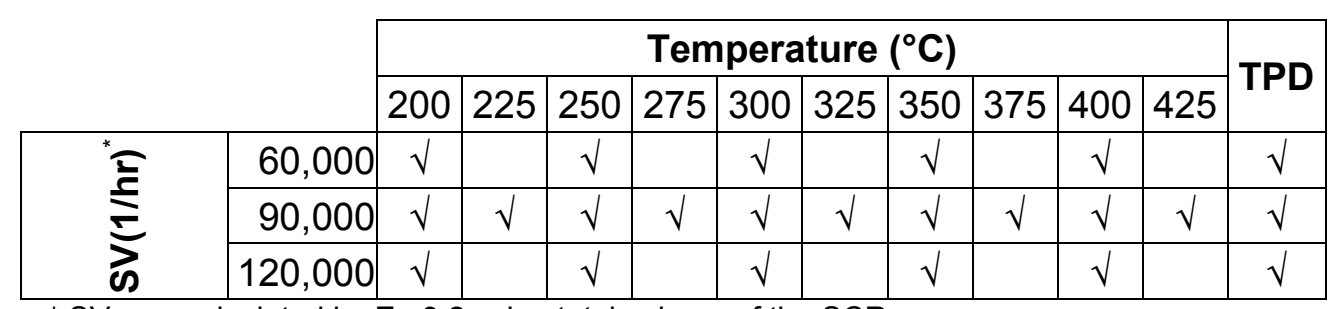

* SV was calculated by Eq 3.2 using total volume of the SCR 


\subsection{Spaci-IR Reactor Tests}

The Spaci-FTIR testing was also developed and carried out at Oak Ridge National Laboratory to measure axially resolved concentrations internal to the SCR channels. The Cu-zeolite core SCR samples, $5 \mathrm{~cm}$ long with a diameter of $2 \mathrm{~cm}$, were taken from the 2010 Cummins ISB production catalyst. The core samples were also de-greened at $700^{\circ} \mathrm{C}$ for four hours under a gas stream containing $20 \% \mathrm{O}_{2}$ and $4.5 \% \mathrm{H}_{2} \mathrm{O}$ and the balance being $\mathrm{N}_{2}$. The core sample was mounted in a micro flow bench-reactor system for reactor testing. The exhaust flow going through the core sample was generated from gas cylinders using mass flow controllers to control the flow rate of each gas. A furnace with feedback control was used to maintain the SCR temperature. A test protocol with nine steps is given in Figure 3.23. The details about the inlet conditions of each step of the protocol are shown in Table 3.17. The protocol was conducted to study the SCR response of $\mathrm{NH}_{3}$ saturation (step 2 of the protocol), $\mathrm{NH}_{3}$ inventory under $\mathrm{NO}_{\mathrm{X}} \mathrm{SCR}$ condition (step 4: $\mathrm{NO}_{2} / \mathrm{NO}_{\mathrm{x}}=0.5$ ), and $\mathrm{NH}_{3}$ inventory under $\mathrm{NO}$ SCR condition (step 7: $\mathrm{NO}_{2} / \mathrm{NO}=0$ ). For each step of the protocol, the gas stream also contained $10 \%$ of $\mathrm{O}_{2}$, $5 \%$ of $\mathrm{H}_{2} \mathrm{O}, 5 \%$ of $\mathrm{CO}_{2}$, with the balance being $\mathrm{N}_{2}$.

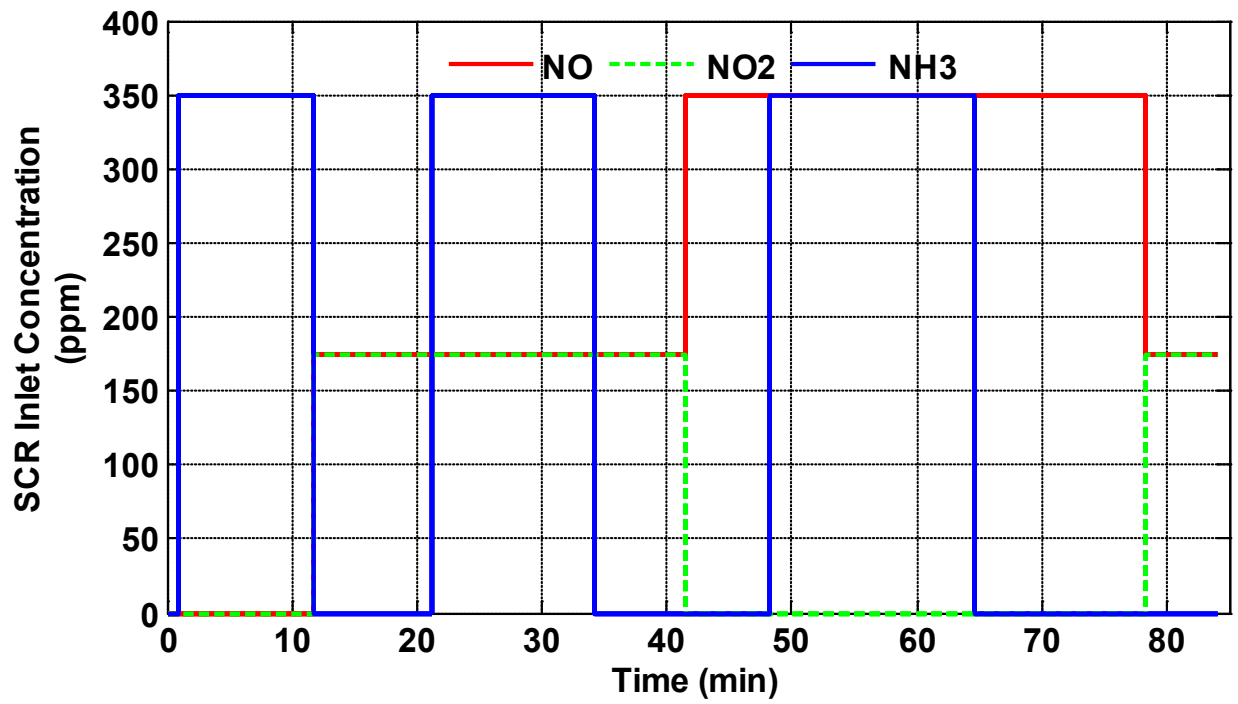

Figure 3.23: Test protocol of the Spaci-IR reactor tests 
Table 3.17: Details of each step of the Spaci-IR test protocol

\begin{tabular}{|c|c|c|c|c|}
\hline Step & Description & NO (ppm) & $\mathrm{NO}_{2}(\mathrm{ppm})$ & $\mathrm{NH}_{3}(\mathrm{ppm})$ \\
\hline 1 & Stabilize & 0 & 0 & 0 \\
\hline 2 & $\mathrm{NH}_{3}$ adsorption & 0 & 0 & 350 \\
\hline 3 & $\mathrm{NH}_{3}$ inventory: $\mathrm{NO}_{2} / \mathrm{NO}_{\mathrm{x}}=0.5$ & 175 & 175 & 0 \\
\hline 4 & $\mathrm{NH}_{3} / \mathrm{NO}_{\mathrm{x}}=1.0 ; \mathrm{NO}_{2} / \mathrm{NO}_{\mathrm{x}}=0.5$ & 175 & 175 & 350 \\
\hline 5 & $\mathrm{NH}_{3}$ inventory: $\mathrm{NO}_{2} / \mathrm{NO}_{\mathrm{x}}=0.5$ & 175 & 175 & 0 \\
\hline 6 & Stabilize $\mathrm{NO}: \mathrm{NO}_{2} / \mathrm{NO}_{\mathrm{x}}=0.0$ & 350 & 0 & 0 \\
\hline 7 & $\mathrm{NH}_{3} / \mathrm{NO}_{\mathrm{x}}=1.0 ; \mathrm{NO}_{2} / \mathrm{NO}_{\mathrm{x}}=0.0$ & 350 & 0 & 350 \\
\hline 8 & $\mathrm{NH}_{3}$ inventory: $\mathrm{NO}_{2} / \mathrm{NO}_{\mathrm{x}}=0.0$ & 350 & 0 & 0 \\
\hline 9 & Clean: $\mathrm{NO}_{2} / \mathrm{NO}_{\mathrm{x}}=0.5$ & 175 & 175 & 0 \\
\hline
\end{tabular}

Axially resolved gaseous concentrations in the core samples were measured by a FTIR with two capillaries sampling from one channel apart from each other near the center of the core sample. In order to increase the flow rate fed to the FTIR, the reactor pressure was controlled to be 1.25 bar and the capillary flow was diluted using a dilution ratio of about 10:1 with $\mathrm{N}_{2}$ before feeding the gas mixture into the FTIR. The actual concentrations in the reactor were equal to the FTIR measured concentrations times the dilution ratio. For each axial measurement location, the test protocol was conducted continuously. The test conditions were held constant until the measured gaseous concentrations reached a steady state for each step of the protocol. The measurement process was repeated for each axial measurement location and each test condition shown in Table 3.18. Another run was conducted for each test temperature measuring at the location of $0.4 \mathrm{~cm}$ in front of the inlet face to determine the inlet conditions. $\mathrm{NH}_{3}$ storage on the catalyst under $\mathrm{NH}_{3}$ saturation, $\mathrm{NO}_{x} \mathrm{SCR}$, and $\mathrm{NO}$ SCR conditions were calculated by integrating the $\mathrm{NO}_{x}$ converted by stored $\mathrm{NH}_{3}$ after $\mathrm{NH}_{3}$ was turned off during step 3, 5, and 8 respectively.

Table 3.18: Test matrix of the Spaci-IR tests

\begin{tabular}{|c|c|c|}
\hline $\begin{array}{c}\text { Space Velocity } \\
\left(\mathbf{h r}^{-1} \mathbf{)}\right.\end{array}$ & $\begin{array}{c}\text { Temperature } \\
\left.\mathbf{(}^{\circ} \mathbf{C}\right)\end{array}$ & $\begin{array}{c}\text { Normalized Axial Measurement } \\
\text { Location }\end{array}$ \\
\hline 60,000 & 250 & $0.05,0.1,0.2,0.3,0.4,0.6,0.8$ \\
\hline 60,000 & 300 & $0.02,0.05,0.1,0.2,0.3,0.4,0.6,0.8$ \\
\hline 60,000 & 350 & $0.05,0.1,0.2,0.3,0.4,0.6,0.8$ \\
\hline
\end{tabular}


When processing the Spaci-IR data, it was determined that the exhaust flow rate for the SCR channel with the sampling capillary was higher than expected, resulting in a higher local space velocity. The reason was determined to be that the lowered local pressure caused by the sampling flow resulted in more flow into the channel. After comparing the $\mathrm{NH}_{3}$ storage for the same catalyst under the same conditions without the Spaci-IR technique, the actual space velocity of the Spaci-IR tests was estimated to be 70,000 instead of $60,000 \mathrm{hr}^{-1}$. 


\section{Chapter 4.}

\section{SCR Model Development and Calibration}

The high fidelity Cu-zeolite SCR model was developed from an existing Fe-zeolite SCR model which was calibrated to reactor data [23]. The model was modified by changing the mass transfer correlation and adding the $\mathrm{NH}_{3}$ oxidation reaction with the oxidation selectivity to $\mathrm{NO}$ (Reaction 2.21) and the $\mathrm{NO}_{2}$ decomposition reaction (Reaction 2.22) which is the reversible reaction of the NO oxidation reaction. It was then calibrated to the Cu-zeolite SCR experimental data from both SCR reactor and engine experiments. The details of the model as well as the calibration procedures are described in this chapter.

\subsection{SCR Model Overview}

The model considers one single channel which is assumed to be one dimensional and divided into 10 finite elements in the axial direction from inlet to outlet. Three types of states including gas phase, surface phase and storage phase are associated with each axial element of the model. Gas phase refers to the bulk gas flowing through the channel. $\mathrm{NH}_{3}$ is assumed to be the only species that is stored on the catalytic surface. The SCR reactions take place between the species in surface and the storage $\mathrm{NH}_{3}$. The model consists of two $\mathrm{NH}_{3}$ storage sites with different properties. The $1^{\text {st }}$ site supports $\mathrm{NH}_{3}$ adsorption and desorption reactions as well as all of the other SCR reactions. While at the same time, the $2^{\text {nd }}$ site only adsorbs and desorbs $\mathrm{NH}_{3}$. Mass transfer takes places between the species in the gas phase and surface phase. Heat transfer between bulk flow and the substrate and between the substrate and the ambient is included in the model to simulate the SCR outlet temperatures under transient conditions. 


\subsubsection{Mass Transfer Equations}

Mass transfer between the gas phase and surface phase is included in the model. The mass balance equations for the gas phase and surface phase is given in Equations 4.1 and 4.2. Equation 4.1 describes the mass balance for the gas phase, and Equation 4.2 describes the mass balance for the surface phase.

$$
\begin{gathered}
\varepsilon \frac{\partial C_{g, i}^{n}}{\partial t}=-u \frac{\partial C_{g, i}^{n}}{\partial x}-\beta_{i} A_{g}\left(C_{g, i}^{n}-C_{s, i}^{n}\right) \\
(1-\varepsilon) \frac{\partial C_{s, i}^{n}}{\partial t}=\beta_{i} A_{g}\left(C_{g, i}^{n}-C_{s, i}^{n}\right)-\sum_{j} N_{i, j} R_{j} \\
i=\mathrm{NO}, \mathrm{NO}_{2}, \mathrm{NH}_{3}, \\
j=\text { ads, des, fast } \mathrm{SCR}, \text { standard SCR, slow SCR, etc. }
\end{gathered}
$$

Where, $\varepsilon$ is the void fraction of the catalyst, $u$ is the speed of the exhaust flow, $A_{g}$ is the geometric surface area and $\beta_{i}$ is the mass transfer coefficient shown in Equation 4.3. $C_{g}$ and $C_{s}$ are the gas phase and surface phase gaseous concentrations.

$$
\beta_{i}=S h_{i} D_{m, i} / d_{h}
$$

Where, $d_{h}$ is the hydraulic diameter of the channel and $D_{m, i}$ is the molecular diffusivity of the $i$ th species. $S h_{i}$ is the Sherwood number of the ith species and is shown in Equation 4.4 [103].

$$
S h_{i}=0.766 \times\left(\frac{d}{L} \cdot R e \cdot S c\right)^{0.483}
$$

Where, $d$ and $L$ are the diameter and length of the channel. Re and $S c$ are the Reynolds number and Schmidt number. A multiplier factor of 4 was applied to the Sherwood number calculation when calibrating the model to better simulate the space velocity effects on the SCR performance. 


\subsection{2 $\mathrm{NH}_{3}$ Storage Equations}

The model assumes $\mathrm{NH}_{3}$ is the only species that can be stored on the catalyst and the stored $\mathrm{NH}_{3}$ react with other species in the surface phase. The storage equations for the two sites of the model are given in Equation 4.5.

$$
\left\{\begin{array}{l}
\Omega_{1} \theta_{1}=R_{A d s 1}-R_{D e s 1}-\sum_{j} N_{j} R_{j} \\
\Omega_{2} \theta_{2}=R_{A d s 2}-R_{D e s 2}
\end{array}\right.
$$

Where, $j$ represents the reaction consuming the stored $\mathrm{NH}_{3} . \theta_{i}$ and $\Omega_{i}(i=1,2)$ are the $\mathrm{NH}_{3}$ coverage fraction and the $\mathrm{NH}_{3}$ storage capacity of each site. $R$ represents the reaction rate for each reaction.

\subsubsection{Heat Transfer Equations}

In order to predict the axial temperature gradients in the SCR as well as the outlet temperatures under transient conditions, the SCR model also includes heat transfer between the exhaust flow and the substrate as well as the heat transfer to the ambient. This is valuable for improving the model prediction performance under transient conditions by considering the axial temperature gradients in the model. The heat transfer equations for the SCR are given in Equations 4.6 and 4.7. Equation 4.6 is the gas phase energy balance equation and Equation 4.7 is the surface phase energy balance equation. The heat transfer to the ambient is empirical and is modeled through convection heat transfer from the outer surface of the channel with the heat transfer coefficient $h_{a}$ and an ambient temperature $T_{a}$. The heat release due to the SCR reactions is negligible and was set to zero in this model.

$$
\rho C_{v} \frac{d T_{g}}{d t}=-\rho u \frac{d T_{g}}{d x}-h_{g} \frac{4}{a_{w}}\left(T_{g}-T_{w}\right)
$$




$$
\rho_{w} C_{w} \frac{d T_{w}}{d t}=h_{g} \frac{4 a_{w}}{a_{p}^{2}-a_{w}^{2}}\left(T_{g}-T_{w}\right)-h_{a} \frac{4 a_{p}}{a_{p}^{2}-a_{w}^{2}}\left(T_{w}-T_{a}\right)+\sum_{i} \frac{-\Delta H_{i} R_{i}}{M W_{i}}
$$

In equations 4.6 and 4.7, $\rho$ and $\rho_{w}$ refer to the density of the exhaust gas and the substrate wall. $T_{w}, T_{g}$, and $T_{a}$ refer to the temperatures of the wall, gas, and the ambient. $h_{g}$ and $h_{a}$ refer to the heat transfer coefficients. $a_{p}$ and $a_{w}$ are the width of the monolith element and the open channel respectively.

\subsubsection{Reaction Mechanism}

The global reactions considered in the model are given in Table 4.1. It was assumed that the reactions were first order in the reactants. The assumption was previously tested against Fe-zeolite SCR reactor data [23]. As can be seen from the table, there were two $\mathrm{NH}_{3}$ storage sites with both sites supporting $\mathrm{NH}_{3}$ adsorption and desorption reactions but only the 1 st site supporting all the other SCR reactions. The $2^{\text {nd }}$ site performed as a storage site which only adsorbs and desorbs $\mathrm{NH}_{3}$. Other reactions taking place on the $1^{\text {st }}$ site included two $\mathrm{NH}_{3}$ oxidation reactions with different selectivity to $\mathrm{N}_{2}$ and $\mathrm{NO}$ respectively, two standard SCR reactions with different equivalence ratio between $\mathrm{NH}_{3}$ and NO in the reactants, the fast SCR reaction, the slow SCR reaction, NO oxidation (the reverse of which is $\mathrm{NO}_{2}$ decomposition), and an $\mathrm{N}_{2} \mathrm{O}$ formation reaction.

R1 and 2 are the $\mathrm{NH}_{3}$ adsorption and desorption reactions on the $1^{\text {st }}$ storage site. R3 and 4 are the $\mathrm{NH}_{3}$ adsorption and desorption reactions on the $2^{\text {nd }}$ storage site. $\mathrm{R} 5$ to 12 are the SCR reactions that take place in the $1^{\text {st }}$ storage site. There are two $\mathrm{NH}_{3}$ oxidation reactions (R5 and 6) showing $\mathrm{NH}_{3}$ oxidation selectivity to both $\mathrm{N}_{2}$ and NO. R7 and 8 are two standard SCR reactions which have different stoichiometry $\mathrm{NH}_{3} / \mathrm{NO}_{x}$ ratio. The $2^{\text {nd }}$ standard SCR reaction (R8) had a higher stoichiometric $\mathrm{NH}_{3}$ to $\mathrm{NO}_{x}$ ratio and was used to explain the $\mathrm{NH}_{3}$ overconsumption observed from the reactor test results. $\mathrm{R} 9$ and 10 are the fast and slow SCR reactions respectively. R11 is the NO oxidation reaction which is considered as a reversible reaction of the $\mathrm{NO}_{2}$ decomposition reaction. $\mathrm{R} 12$ is the $\mathrm{N}_{2} \mathrm{O}$ formation from $\mathrm{NO}_{2}$ and the stored $\mathrm{NH}_{3}$. The reaction rate calculation for each 
reaction is also given in the table. The reaction rate constant was assumed to be in Arrhenius form as shown in Equation 4.8.

$$
k=A e^{-\frac{E}{R T}}
$$

Where, $A$ and $E$ are the pre-exponential factor and activation energy. $R$ is the universal gas constant and $T$ is the temperature.

The $2^{\text {nd }}$ standard SCR reaction (R8 in Table 4.1) which has a higher $\mathrm{NH}_{3} / \mathrm{NO}$ stoichiometry was added in the reaction mechanism to account for the overconsumption of $\mathrm{NH}_{3}$ with respect to $\mathrm{NO}$ observed in reactor tests as given in Figure 4.1. The figure shows the molar ratio of consumed $\mathrm{NH}_{3}$ and $\mathrm{NO}$ during step 11 (ANR = 1.2) and 13 $(A N R=1.0)$ of the reactor test protocol shown in Figure $3.21 \mathrm{It}$ can be observed that this ratio is slightly higher than unity in the temperature range of 200 to $400^{\circ} \mathrm{C}$, meaning that more $\mathrm{NH}_{3}$ was consumed than the $\mathrm{NO}$ reduced. Similar $\mathrm{NH}_{3}$ overconsumption phenomena were reported for both Fe-zeolite and Cu-zeolite SCR [63,87], and it was explained by the $\mathrm{NH}_{3}$ oxidation reactions that consume $\mathrm{NH}_{3}$. The reactor experimental data during step 3 of the test protocol showed that the $\mathrm{NH}_{3}$ oxidation reactions were not active at temperatures under $250^{\circ} \mathrm{C}$ as shown in Figure 4.1. The $\mathrm{NH}_{3}$ overconsumption was obvious in this temperature range. As a result, the reaction R8 which has a higher $\mathrm{NH}_{3} / \mathrm{NO}$ stoichiometry was added into the SCR reaction mechanism. 


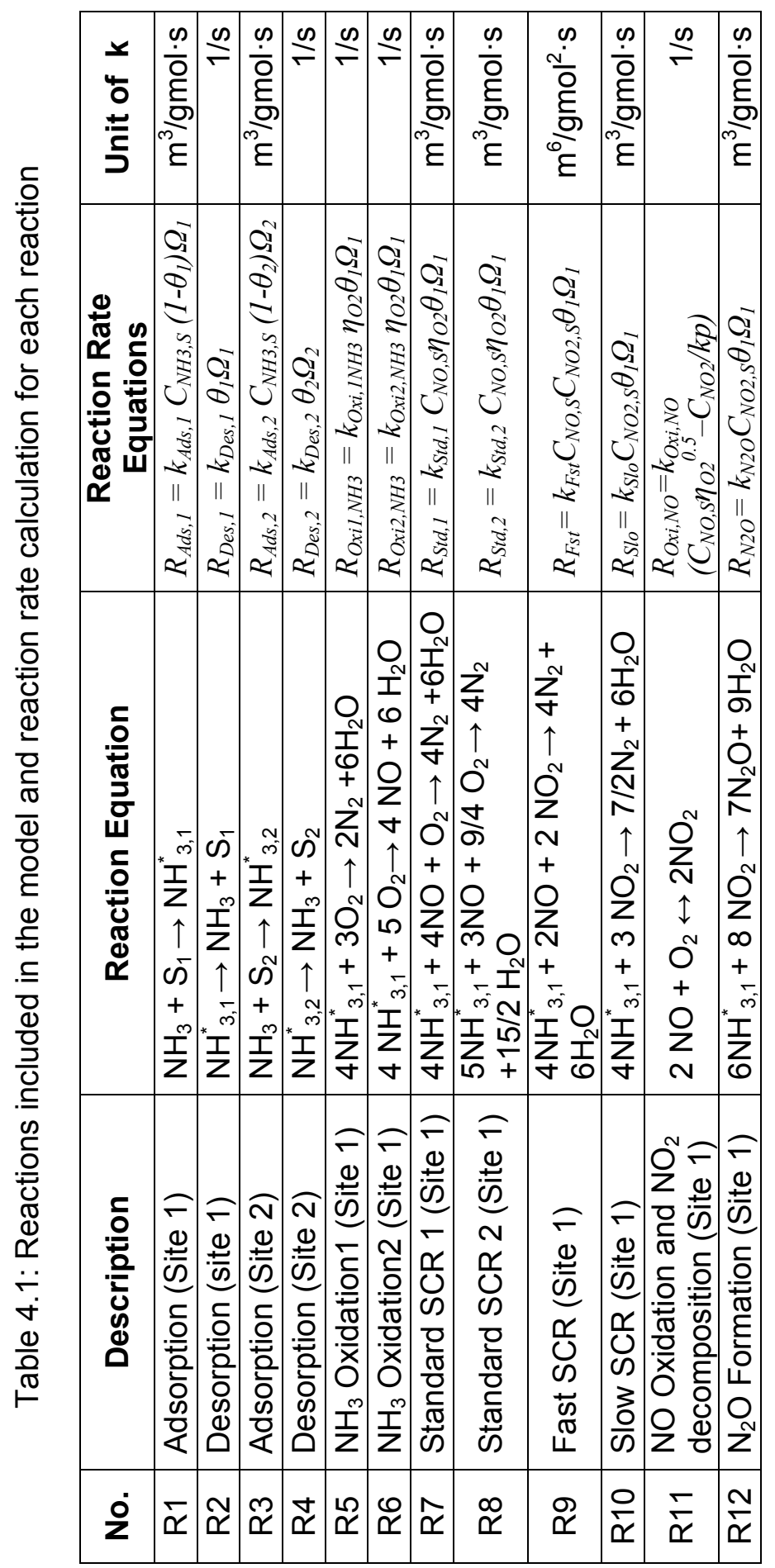




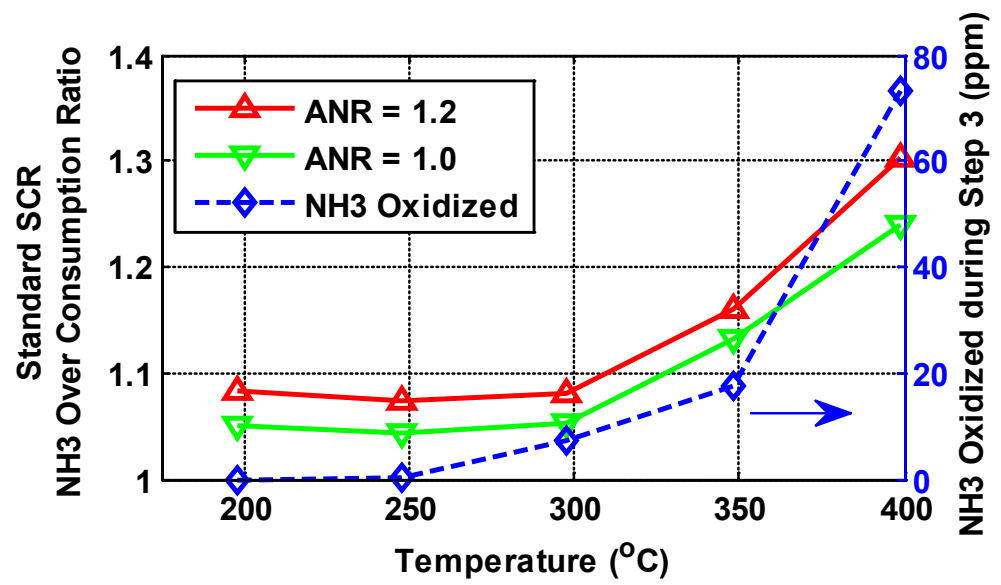

Figure 4.1: Overconsumption of $\mathrm{NH}_{3}$ with respect to $\mathrm{NO}$ under standard SCR conditions and $\mathrm{NH}_{3}$ oxidized during step 3 (Space Velocity $=60,000 \mathrm{hr}^{-1}$ )

Expressions for the reaction rate for each reaction included in the model and the corresponding units of the reaction rate constant $k$ are also given in Table 4.1. $S_{1}$ and $S_{2}$ represent the two storage sites; $\mathrm{NH}_{3,1}^{*}$ and $\mathrm{NH}_{3,2}^{*}$ are the $\mathrm{NH}_{3}$ adsorbed on the catalytic surface of each site. $\theta_{i}$ and $\Omega_{i}(i=1,2)$ are the $\mathrm{NH}_{3}$ coverage fraction and the $\mathrm{NH}_{3}$ storage capacity of each site. $R$ and $k$ represent the reaction rate and the reaction rate constant for each reaction. $\eta_{\mathrm{O} 2}$ is the oxygen concentration in terms of a mole fraction. The equation for calculating the reaction rate constant is given as Equation 4.9.

$$
k=A \cdot \exp (-E / R T)
$$

Where, $A$ is the pre-exponential and $E$ is the activation energy for each reaction, $R$ is the universal gas constant, $T$ is the temperature.

\subsection{SCR Model Calibration with Spaci-IR Data}

The Spaci-IR data provided good insights about the SCR internal states including gaseous concentrations and the $\mathrm{NH}_{3}$ storage along the catalyst channel. Only SCR inlet and outlet concentrations were collected from the traditional reactor tests and the SCR internal states were unknown. It was difficult to validate the internal state predictions 
from a model capable of correctly predicting the outlet concentrations. The model performance in terms of internal states predictions can be verified by the Spaci-IR test results. Furthermore, instead of calibrating the model to simulate the time history of the SCR outlet species concentrations, the Spaci-IR experimental data provided a novel approach to quantify the relationships between the critical kinetic model parameters.

As can be seen from the SCR reaction equations, the $\mathrm{NH}_{3}$ stored on the catalyst participates in all SCR reactions. As a result, the $\mathrm{NH}_{3}$ storage capacity of each model site $\Omega_{i}(i=1,2)$ are key parameters that need to be identified first. The total $\mathrm{NH}_{3}$ storage capacity of the catalyst can be calculated by integrating the $\mathrm{NO}_{\mathrm{x}}$ reduced by stored $\mathrm{NH}_{3}$ during step 3 of the protocol. Since there were two storage sites in the model, how to separate the total storage capacity between each site became a unique problem to solve. The gas phase concentrations were assumed to be equal to the surface phase concentrations in this analysis. For the purpose of simplifying the calculation, a 1-site model was used to compute the total ammonia storage capacity.

\subsubsection{1-Site Model Analysis}

The steady-state portion of the experimental data at step 2 of the protocol shown in Figure 3.23 and Table 3.17, which is the $\mathrm{NH}_{3}$ saturation section, was used for the storage analysis. The time rate of change of stored $\mathrm{NH}_{3}$ was zero and the reaction rate of the adsorption reaction was equal to that of the desorption reaction when the catalyst reached the equilibrium state. This leads to Equation 4.10 in which the left hand side is the $\mathrm{NH}_{3}$ adsorption rate and the right hand side of the equation is the desorption rate. The reaction rate constant for the adsorption and desorption reactions are assumed to be in Arrhenius form as shown in Equation 4.11.

$$
\begin{gathered}
k_{a d s, i} C_{N H 3, i}\left(1-\theta_{i}\right) \Omega=k_{d e s, i} \theta_{i} \Omega \\
k_{j}=A_{j} \exp \left(-E_{j} / R T_{i}\right)
\end{gathered}
$$


Where, the subscript $i$ represents the $i$ th test temperature $\left(250,300\right.$, or $\left.350^{\circ} \mathrm{C}\right)$ and $j$ is reaction index. The quantity $k$ is the reaction rate constant for the reactions, $C$ is the gas concentration, $\theta$ is the $\mathrm{NH}_{3}$ coverage fraction which is a ratio of the $\mathrm{NH}_{3}$ storage at each temperature and $\Omega_{i}$ is the storage capacity $\Omega$, shown in Equation 4.12.

$$
\theta_{i}=\Omega_{i} / \Omega
$$

The relationship between the reaction rate constants of adsorption and desorption reactions given in Equation 4.13 can be derived by substituting Equation 4.12 into Equation 4.10 and rearranging the equation.

$$
k_{a d s, i} / k_{d e s, i}=\Omega_{i} /\left(C_{N H 3, i}\left(\Omega-\Omega_{i}\right)\right)
$$

The left hand side of Equation 4.13 can be rearranged by replacing the reaction rate constants by Equation 4.11 for $\mathrm{NH}_{3}$ adsorption and desorption reactions shown in Equation 4.14. As a result, Equation 4.15 can be obtained from Equations 4.13 and 4.14.

$$
\begin{gathered}
k_{a d s, i} / k_{d e s, i}=\frac{A_{a d s} \exp \left(-E_{a d s} / R T_{i}\right)}{A_{d e s} \exp \left(-E_{d e s} / R T_{i}\right)}=\frac{A_{a d s}}{A_{d e s}} \exp \left(-\frac{E_{a d s}-E_{d e s}}{R T_{i}}\right) \\
\frac{A_{a d s}}{A_{d e s}} \exp \left(-\frac{E_{a d s}-E_{d e s}}{R T_{i}}\right)=\Omega_{i} /\left(C_{N H 3, i}\left(\Omega-\Omega_{i}\right)\right)
\end{gathered}
$$

Equation 4.16 was derived by taking the natural log of both sides of Equation 4.15 and rearranging the equation. Three unknown terms showing the relationships between the pre-exponentials and activation energies of the $\mathrm{NH}_{3}$ adsorption and desorption relations as well as the storage capacity $\Omega$ are present in the equation. The $\mathrm{NH}_{3}$ concentrations $C_{N H 3}$ and the $\mathrm{NH}_{3}$ storage $\Omega_{i}$ at different axial positions of the catalyst can be quantified from the Spaci-IR test results for the three temperatures tested $\left(250,300\right.$ and $\left.350^{\circ} \mathrm{C}\right)$. The procedures for the $\mathrm{NH}_{3}$ storage calculation are shown in Appendix $\mathrm{F}$. Three identical equations can be obtained from Equation 4.16 for the three temperatures tested. The three unknown terms in Equation 4.16 can be quantified by solving the equation set for the three temperature runs. 


$$
\ln \left(\frac{A_{a d s}}{A_{d e s}}\right)-\frac{\left(E_{a d s}-E_{d e s}\right)}{R T_{i}}=\ln \left(\frac{\Omega_{i}}{C_{N H 3, i}}\right)-\ln \left(\Omega-\Omega_{i}\right)
$$

The calculated $\Omega_{i}$ for 250,300 , and $350^{\circ} \mathrm{C}$ are $\Omega_{1}=84.4, \Omega_{2}=63.8$, and $\Omega_{3}=37.7$ $\mathrm{gmol} / \mathrm{m}^{3}$ as shown in Figure 4.2. A linear relationship between $\mathrm{NH}_{3}$ storage and temperature in this temperature range for the same catalyst formation was reported when $\mathrm{O}_{2}$ was not present in the stream. $\Omega_{3}$ was below the dotted red line generated from $\Omega_{1}$ and $\Omega_{2}$ because $\mathrm{NH}_{3}$ was oxidized at the temperature of $350^{\circ} \mathrm{C}$. The $\mathrm{NH}_{3}$ oxidation resulted in less $\mathrm{NH}_{3}$ storage than expected. In order to eliminate the effect of $\mathrm{NH}_{3}$ oxidation, $\Omega_{3}{ }^{*}=43.1 \mathrm{gmol} / \mathrm{m}^{3}$ which fell on the line from the two lower temperature tests was used. After substituting the known parameters for each temperature, a set of three equations were available for solving the three unknown terms. One meaningful solution was shown in Table 4.2. The $\mathrm{NH}_{3}$ adsorption reaction was typically considered as nonactivated reaction with activation energy of zero. As a result, the activation energy for desorption reaction is equal to $41.3 \mathrm{~kJ} / \mathrm{gmol}$.

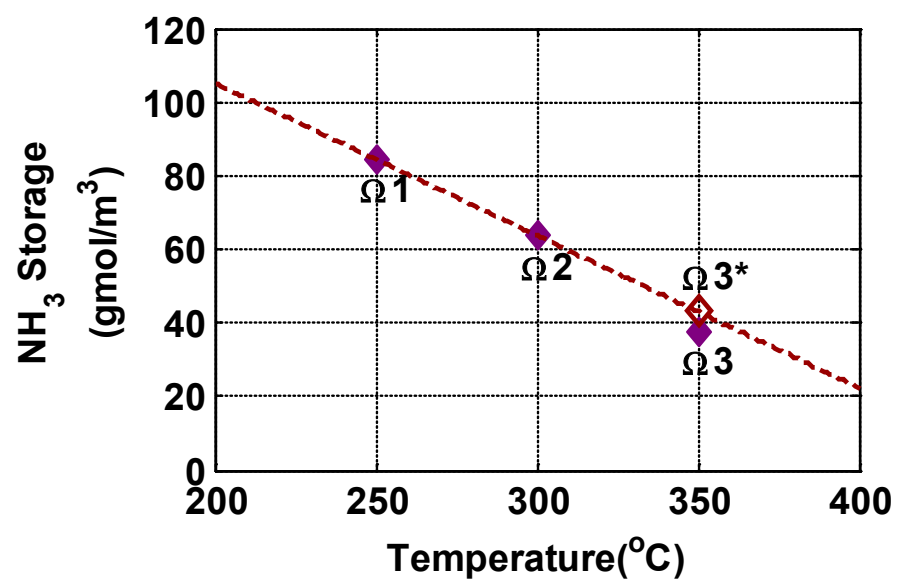

Figure 4.2: $\mathrm{NH}_{3}$ storage at each test temperature

Table 4.2: Identified $\mathrm{NH}_{3}$ storage capacity, Aads/Ades and Eads-Edes for 1-site model

\begin{tabular}{|l|c|c|}
\hline Parameter & Value & Unit \\
\hline $\boldsymbol{\Omega}$ & 108 & $\mathrm{gmol} / \mathrm{m}^{3}$ \\
\hline Aads $/$ Ades & $2.84 \mathrm{e}-2$ & $\mathrm{none}$ \\
\hline Eads - Edes & 41.3 & $\mathrm{~kJ} / \mathrm{gmol}$ \\
\hline
\end{tabular}


The $\mathrm{NH}_{3}$ oxidation at $350^{\circ} \mathrm{C}$ was used to calculate the relationship between the reaction rate constant of $\mathrm{NH}_{3}$ adsorption reaction and $\mathrm{NH}_{3}$ oxidation to $\mathrm{N}_{2}$. The $2^{\text {nd }} \mathrm{NH}_{3}$ oxidation reaction was not considered because it was reported that the $\mathrm{NH}_{3}$ oxidation to $\mathrm{NO}$ is not significant below a temperature of $550^{\circ} \mathrm{C}$ [94]. By considering the $\mathrm{NH}_{3}$ oxidation reaction, the $\mathrm{NH}_{3}$ storage equation can be written as shown in Equation 4.17. Equation 4.18 can be derived by substituting reaction rate equations of $\mathrm{NH}_{3}$ adsorption, desorption and oxidation reactions as well as Equation 4.12 into Equation 4.17. With the identified relationships above, the ratio of the reaction rate constant for $\mathrm{NH}_{3}$ adsorption and oxidation reaction can be quantified by solving Equations $4.16(i=3)$ and 4.18. The calculation results showed that $k_{\text {ads }} / k_{\text {oxil }}=1.63$ at the temperature of $350{ }^{\circ} \mathrm{C}$.

$$
\begin{gathered}
\Omega \theta=R_{a d s}-R_{d e s}-4 R_{o x i 1}=0 \\
k_{a d s, 3} C_{N H 3,3}\left(1-\frac{\Omega_{3}}{\Omega}\right)-k_{d e s, 3} \frac{\Omega_{3}}{\Omega}=4 k_{o x i 1,3} \eta_{o 2} \frac{\Omega_{3}}{\Omega}
\end{gathered}
$$

The next step was to calculate the activation energy and pre-exponential constant of the fast SCR reaction using steady state portions of step 4 and $5\left(\mathrm{NO}_{\mathrm{x}}+\mathrm{NH}_{3} \mathrm{SCR}\right.$ condition). Fast SCR reaction was dominant with a $\mathrm{NO}_{2} / \mathrm{NO}_{x}$ ratio equal to 0.5 . Other SCR reactions were not considered under this condition in order to simplify the calculation. The changing rates of gaseous concentrations for both gas and surface phase are zero under the SCR equilibrium state, meaning that the concentrations at different axial positions don't change with time when reaching the equilibrium state. Under this condition, Equation 4.19 can be derived from the mass balance equations for both gas and surface phases shown in Equations 4.1 and 4.2 based on the assumption that the gas phase concentrations are equal to the surface phase concentrations.

$$
-u \frac{\partial C_{g, i}}{\partial x}=\sum_{j} N_{i, j} R_{j}
$$

Based on the assumption that only the fast SCR reaction takes place under this condition, Equation 4.19 can be rewritten as given in Equation 4.20. The reaction rate equation for the fast SCR reaction is shown in Equation 4.21. 


$$
\begin{gathered}
-u \frac{\partial C_{g, N H_{3}}}{\partial x}=-2 u \frac{\partial C_{g, N O}}{\partial x}=-2 u \frac{\partial C_{g, N O_{2}}}{\partial x}=R_{a d s}-R_{d e s}=4 R_{f s t} \\
R_{f s t}=k_{f s t} C_{N O, s} C_{N_{2}, s} \Omega \theta
\end{gathered}
$$

Axially resolved measurements of $\mathrm{NH}_{3}, \mathrm{NO}$, and $\mathrm{NO}_{2}$ provided rich data for calculating the fast SCR reaction rate constants through Equations 4.20 and 4.21. $k_{f s t}$ on each axial element divided by two axial measurement locations was calculated using the axial measurements under each temperature for $\mathrm{NH}_{3}, \mathrm{NO}$, and $\mathrm{NO}_{2}$ respectively. The calculated $k_{f s t}$ at 3 temperatures for each element was then plotted in Arrhenius form for the purpose of determining the activation energy and pre-exponential constant. The results was $E_{f s t} \cong 53.8 \mathrm{~kJ} / \mathrm{gmol}$, the $A_{f s t}$ was not constant along the axial locations and ranged from $1.63 \mathrm{e} 8$ to $1.36 \mathrm{e} 9 \mathrm{~m}^{6} / \mathrm{gmol}^{2}$.s with low values at the front of the catalyst and high values at the end of the catalyst.

With the identification results presented above, the 1-site SCR model was run with a different set of parameters following the identified values and relationships between parameters. It was found that a one storage site model was not able to correctly simulate both axial gas concentrations and $\mathrm{NH}_{3}$ storage distributions. After tuning the model parameters following the relationships determined above, the model was able to make correct predictions of axial concentrations under the $\mathrm{NO}_{x}+\mathrm{NH}_{3} \mathrm{SCR}$ section. The model simulated $\mathrm{NO}_{x}$ and $\mathrm{NH}_{3}$ concentrations at different axial locations of the catalyst compared to experimental results are given in Figures 4.3 and 4.4. 


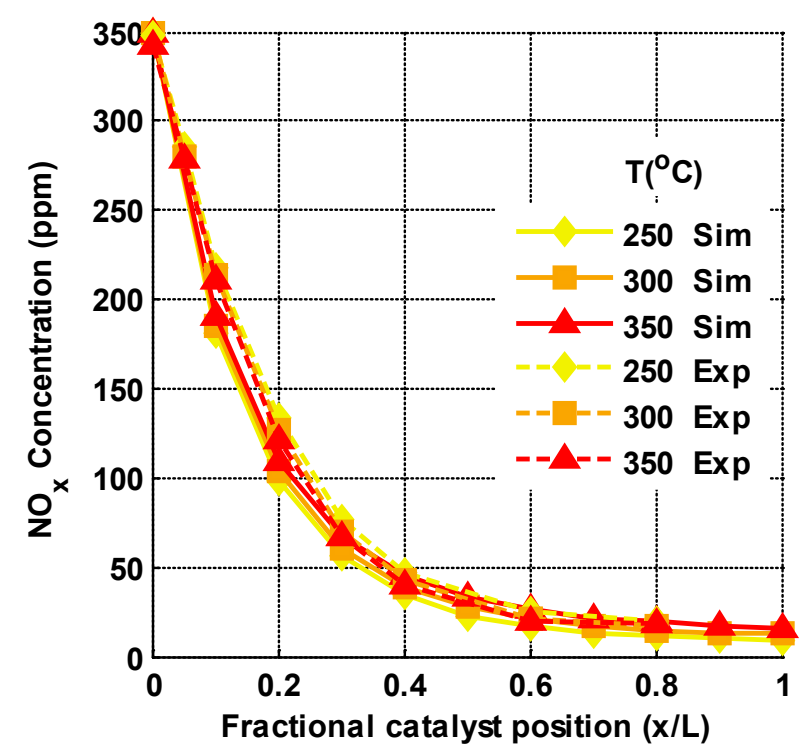

Figure 4.3: 1 -site model simulated $\mathrm{NO}_{x}$ concentration at different axial locations at equilibrium state of $\mathrm{NO}_{\mathrm{x}}+\mathrm{NH}_{3}$ reaction section compared to experimental results

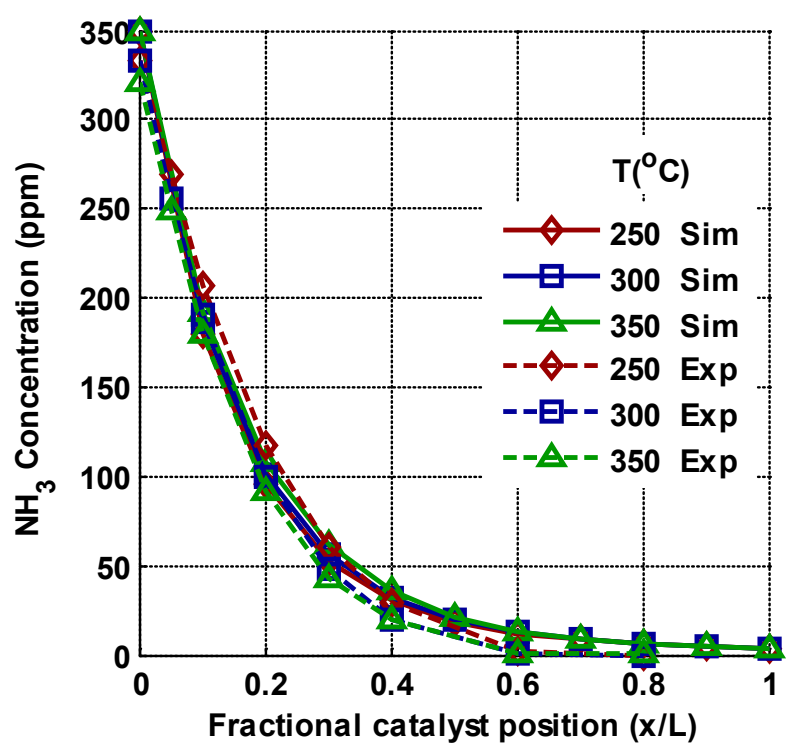

Figure 4.4: 1-site model simulated $\mathrm{NH}_{3}$ concentration at different axial locations at equilibrium state of $\mathrm{NO}_{\mathrm{x}}+\mathrm{NH}_{3}$ reaction section compared to experimental results

The $\mathrm{NH}_{3}$ storage as a function of axial location under $\mathrm{NH}_{3}$ saturation and $\mathrm{NO}_{\mathbf{x}}+\mathrm{NH}_{3}$ sections is given in Figure 4.5. It can be seen that the storage capacity under $\mathrm{NH}_{3}$ 
saturation condition was correctly predicted. The $\mathrm{NH}_{3}$ storage during the $\mathrm{NO}_{\mathrm{x}}+\mathrm{NH}_{3}$ SCR condition was underestimated. This was because the $\mathrm{NH}_{3}$ being adsorbed on the catalyst under the $\mathrm{NO}_{x} \mathrm{SCR}$ condition was consumed more quickly than expected through SCR reactions at the same time. Reducing the reaction rate of the fast SCR reaction can compensate for the $\mathrm{NH}_{3}$ storage during the $\mathrm{NO}_{\mathrm{x}} \mathrm{SCR}$ condition. It led to prediction errors in gaseous concentrations. The solution to correct the $\mathrm{NH}_{3}$ storage prediction without affecting the gas concentration prediction was to add the $2^{\text {nd }}$ storage site which supported only $\mathrm{NH}_{3}$ adsorption and desorption.
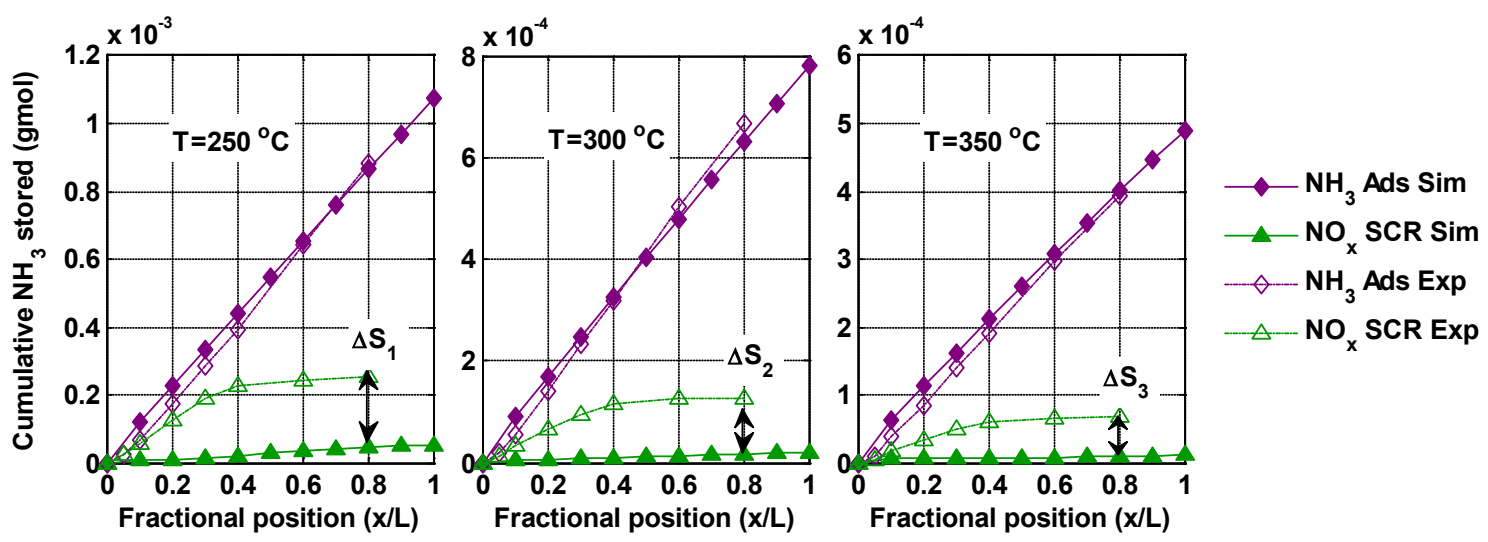

Figure 4.5: Simulated cumulative $\mathrm{NH}_{3}$ stored for the 1-site model as a function of axial position compared to experimental results

\subsubsection{2-Site Model Analysis}

As shown in Figure 4.5, the differences in $\mathrm{NH}_{3}$ storage between model simulation and experimental results under $\mathrm{NO}_{x}+\mathrm{NH}_{3} \mathrm{SCR}$ condition $(\Delta \mathrm{S} 1, \Delta \mathrm{S} 2$ and $\Delta S 3)$ were considered to be the $\mathrm{NH}_{3}$ stored on the $2^{\text {nd }}$ site. As a result, the $\mathrm{NH}_{3}$ storage for each temperature on the $2^{\text {nd }}$ site was calculated to be $34.7,21.1$ and $7.6 \mathrm{gmol} / \mathrm{m}^{3}$. By solving the set of equations derived from Equation 4.16 for the $2^{\text {nd }}$ site at three temperatures, the storage capacity $\Omega_{2}, A_{a d s 2} / A_{d e s 2}$, and $E_{a d s 2}-E_{d e s 2}$ can be solved The meaningful solution for this set of equations was $\Omega_{2}=39.1 \mathrm{gmol} / \mathrm{m}^{3}, A_{a d s 2} / A_{d e s 2}=1.66 \mathrm{e}-6$, and $E_{a d s 2}-E_{d e s 2}=-$ 
$89.2 \mathrm{~kJ} / \mathrm{gmol}$. Similarly, the adsorption reaction on the $2^{\text {nd }}$ site was considered as a nonactivated reaction, and $E_{a d s 2}$ was set to zero, resulting in $E_{\text {des } 2}=89.2 \mathrm{~kJ} / \mathrm{gmol}$.

Adding the $2^{\text {nd }}$ site without reducing the $\mathrm{NH}_{3}$ storage capacity of the $1^{\text {st }}$ site resulted in overestimation of the $\mathrm{NH}_{3}$ stored during the $\mathrm{NH}_{3}$ saturation section of the test protocol. The $\mathrm{NH}_{3}$ storage capacity of site 1 needs to be reduced after adding the $2^{\text {nd }}$ site. The 2site model with identified parameters above was run to determine how much $\mathrm{NH}_{3}$ was actually stored on the $2^{\text {nd }}$ site. Then the difference in $\mathrm{NH}_{3}$ storage between the $\mathrm{NH}_{3}$ stored on the $2^{\text {nd }}$ site and the experimentally found storage was considered to be the storage on the $1^{\text {st }}$ site.

Simulation results showed that about $39.5,30.0$, and $20.5 \mathrm{gmol} / \mathrm{m}^{3}$ of $\mathrm{NH}_{3}$ was stored on the $2^{\text {nd }}$ site under $\mathrm{NH}_{3}$ saturation condition for 250,300 , and $350{ }^{\circ} \mathrm{C}$ respectively. The differences between storage values on the $2^{\text {nd }}$ site and the experimentally found values shown in Figure 4.2 were 44.9, 33.8, and $17.2 \mathrm{gmol} / \mathrm{m}^{3}$ for 250,300 , and $350^{\circ} \mathrm{C}$ respectively, and those values were considered as the $\mathrm{NH}_{3}$ storage on the $1^{\text {st }}$ site after adding the $2^{\text {nd }}$ site. Then the storage capacity of the $1^{\text {st }}$ site $\left(\Omega_{1}\right), A_{\text {adsl }} / A_{\text {des } I}$, and $E_{\text {ads } 1}-E_{d e s I}$ were also updated through re-solving the equation set derived from Equation 4.16 for each temperature. The solution was: $\Omega_{1}=55.5 \mathrm{gmol} / \mathrm{m}^{3}, A_{\text {ads }} / A_{\text {des } 1}=1.28 \mathrm{e}-2, E_{\text {ads } 1}-E_{\text {des } 1}=$ $-45.4 \mathrm{~kJ} / \mathrm{gmol}$. Again $E_{\text {ads } 1}$ was set to be zero since the $\mathrm{NH}_{3}$ adsorption reaction was considered as non-activated reaction. The identified storage parameters and the relationships for the two storage sites are given in Table 4.3.

Table 4.3: Identified $\mathrm{NH}_{3}$ storage capacity, Aads/Ades and Eads-Edes for the storage sites of the 2-site model

\begin{tabular}{|c|r|c|}
\hline Parameter & \multicolumn{1}{|c|}{ Value } & Units \\
\hline$\Omega_{1}$ & 55.5 & $\mathrm{gmol} / \mathrm{m}^{3}$ \\
\hline$\Omega_{2}$ & 39.1 & $\mathrm{gmol} / \mathrm{m}^{3}$ \\
\hline Aads $1 /$ Ades 1 & $1.28 \mathrm{e}-2$ & $\mathrm{none}$ \\
\hline Eads $1-$ Edes 1 & -45.4 & $\mathrm{~kJ} / \mathrm{gmol}$ \\
\hline Aads $2 /$ Ades 2 & $1.66 \mathrm{e}-6$ & $\mathrm{none}$ \\
\hline Eads $2-$ Edes 2 & -89.2 & $\mathrm{~kJ} / \mathrm{gmol}$ \\
\hline
\end{tabular}

After determining the storage parameters for each site, the model kinetic parameters of the remaining reactions taking place on the first site were updated following the 
parameter identification process presented in the previous section. The comparisons between the model simulation results using the parameters constrained by the identified relationships and the experimental results are given in Figures 4.6, 4.7 and 4.8. Figures 4.6 and 4.7 show the comparison of axially resolved $\mathrm{NO}_{x}$ and $\mathrm{NH}_{3}$ concentrations at each temperature between simulation and experimental results under the $\mathrm{NO}_{x} \mathrm{SCR}$ condition of the protocol. The difference in concentrations between temperatures is not significant and the model is able to capture this feature. The simulated concentrations also follow the same trend along the axial location. More than $90 \%$ of the $\mathrm{NO}_{x}$ reduction took place in the first half of the catalyst length. There is not $\mathrm{NH}_{3}$ available for $\mathrm{NO}_{x}$ reduction after the fractional location (x/L) 0.6. A low level of $\mathrm{NO}_{\mathrm{x}}(<20 \mathrm{ppm})$ is still present after this position.

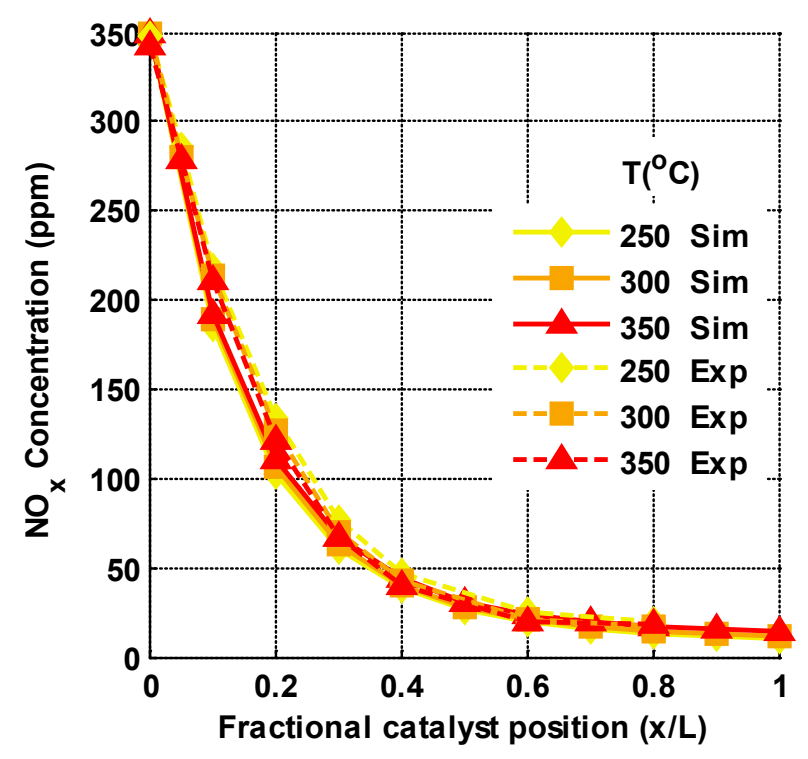

Figure 4.6: 2-site model simulated $\mathrm{NO}_{x}$ concentration at different axial locations at equilibrium state of $\mathrm{NO}_{\mathrm{x}}+\mathrm{NH}_{3}$ protocol section compared to experimental results 


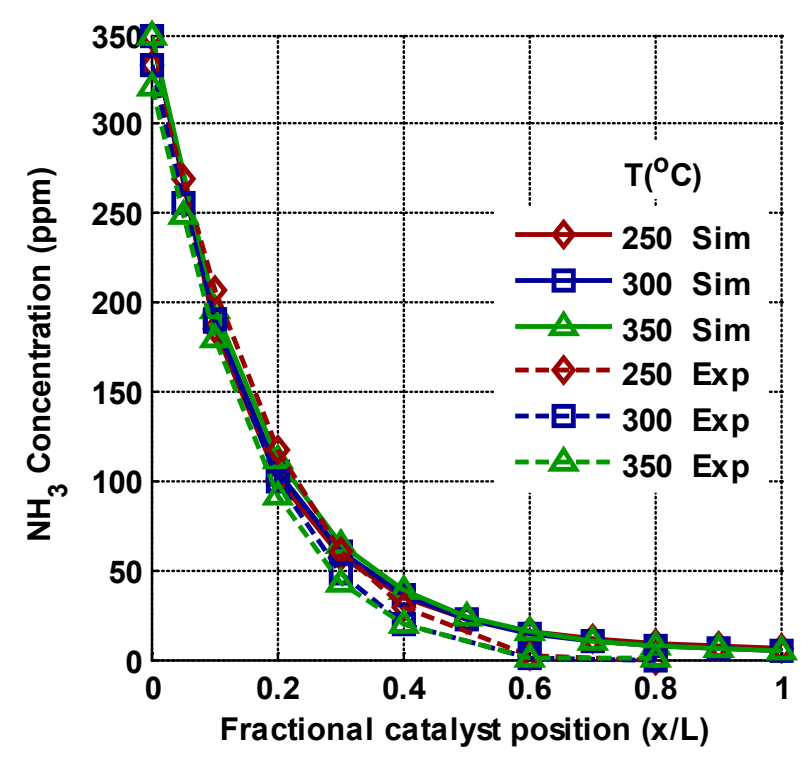

Figure 4.7: 1-site model simulated $\mathrm{NH}_{3}$ concentration at different axial locations at equilibrium state of $\mathrm{NO}_{\mathrm{x}}+\mathrm{NH}_{3}$ protocol section compared to experimental results

Figure 4.8 shows comparisons of the cumulative $\mathrm{NH}_{3}$ stored on the catalyst at each axial measurement location for three temperatures under $\mathrm{NH}_{3}$ saturation and $\mathrm{NO}_{x} \mathrm{SCR}$ conditions of the protocol. The $\mathrm{NH}_{3}$ stored under $\mathrm{NH}_{3}$ saturation condition shows close to a linear increase along the axis for all three temperatures. The model can correctly predict this phenomenon except that it is slightly higher than the experimental results for the $350^{\circ} \mathrm{C}$ data. The storage differences between $\mathrm{NH}_{3}$ saturation and $\mathrm{NO}_{x} \mathrm{SCR}$ conditions are considered to be caused by consumption of stored $\mathrm{NH}_{3}$ through SCR reactions changing the equilibrium state. The experimental results show that the $\mathrm{NH}_{3}$ is completely consumed before fractional position 0.6 . The model gives a slightly different trend for the temperature of $250^{\circ} \mathrm{C}$, but the simulation results show good agreement with the experimental results for higher temperatures of 300 and $350^{\circ} \mathrm{C}$. 

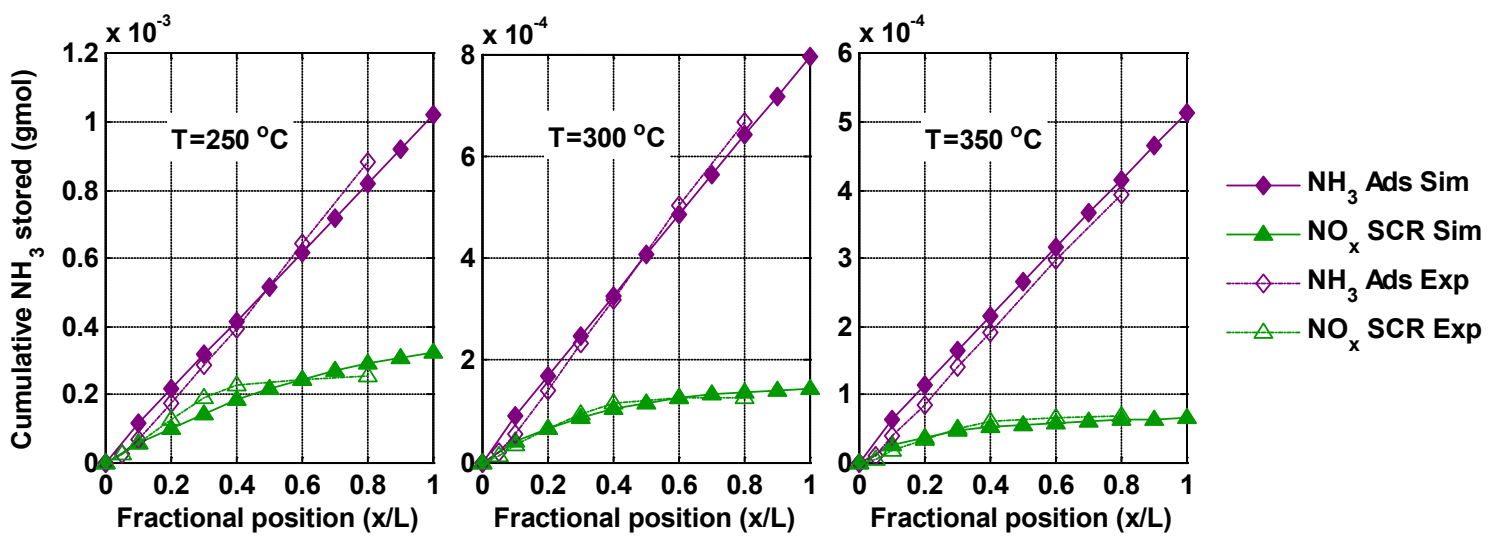

Figure 4.8: Simulated cumulative $\mathrm{NH}_{3}$ stored of the 1-site model as a function of axial position compared to experimental results

The model parameter relationships identified through calibration to the Spaci-IR data served as a basis for developing a SCR model calibration for the reactor data.

\subsection{SCR Model Calibration with Flow Reactor Data}

SCR model parameters including the $\mathrm{NH}_{3}$ storage capacities for each of the two sites, as well as $A$ and $E$ for each of the twelve SCR reactions in Table 4.1 need to be identified by calibrating the model against the reactor experimental data. The SCR model calibration was targeted at matching the model simulated gaseous concentrations with downstream SCR experimental time history data for both the steady state and transient segments of the protocol. Only certain reactor data were selected for model calibration as discussed in Appendix G [128].

\subsubsection{Model Calibration Procedures}

The model parameters from the existing Fe-zeolite SCR model and the relationships quantified from the Spaci-IR data served as the starting point for calibrating the SCR high fidelity model to the reactor data. The reaction rates of the SCR reactions are highly dependent on the $\mathrm{NH}_{3}$ storage capability of the catalyst because the stored $\mathrm{NH}_{3}$ 
participates in all SCR reactions. As a result, the $\mathrm{NH}_{3}$ storage capacities of each site needs to be identified along with the adsorption and desorption reaction rate constant for each test condition as a first step in the model calibration. The next step is to manually tune the reaction rate constant $(k)$ for each SCR reaction to find the best fit between model simulation and experimental measurements for each test condition. Each reaction can be calibrated individually by using the data of certain segments of the test protocol as described in the previous sections. The third step is to carry out an optimization method using the manually found approximate reaction rate constants as a basic calibration. The optimization method called a MATLAB "fmincon" function was used to determine the set of reaction rate constants for the reactions which gave the lowest cost function value. The cost function value for each species is defined as the accumulative absolute error between the model prediction and the experimental measurement divided by the simulation time. The equation calculating the cost function value for each species is given in Equation 4.22.

$$
\operatorname{Cost}_{i}=\frac{\sum_{t_{0}}^{t_{\text {end }}}\left|C_{i, \operatorname{Sim}}-C_{i, \text { Exp }}\right|}{t_{\text {end }}}
$$

Where Cost $t_{i}$ is the cost function for gas species $i\left(i=\mathrm{NO}, \mathrm{NO}_{2}, \mathrm{NH}_{3}\right) . t_{0}$ and $t_{\text {end }}$ are the start and stop time in seconds for the simulation. $\mathrm{C}_{i, \text { sim }}$ and $\mathrm{C}_{i, \text { exp }}$ are the model simulated and experimentally measured gas concentrations for the gas species $i$ respectively. The fourth step is to plot the optimal reaction rate constants for each reaction determined following the optimization process for each test condition described above in Arrhenius form. The process is then to find the linear trend line of the plotted points. The slope and intercept of the trend line were used to calculate the activation energy and preexponential of each reaction using Equation 4.23.

$$
\left\{\begin{array}{l}
A=e^{c} \\
E=-m \cdot R
\end{array}\right.
$$


$m$ is the slope and $c$ is the intercept of the trend line. The confidence interval for the activation energy of each reaction was calculated from the standard error of the slope of each trend line in the Arrhenius plot.

\subsection{2 $\mathrm{NH}_{3}$ Storage Parameters}

$\mathrm{NH}_{3}$ stored on the SCR during step 2 of the protocol was calculated by integrating the difference between the SCR inlet and outlet $\mathrm{NH}_{3}$ concentrations for each temperature and space velocity tested. Calculation results are shown in Figure 4.9. It is clear from the figure that the catalyst stored more $\mathrm{NH}_{3}$ at lower temperatures. The $\mathrm{NH}_{3}$ stored is close to a linear relationship with temperatures between 250 and $350^{\circ} \mathrm{C}$ for each space velocity tested. The highest $\mathrm{NH}_{3}$ storage observed was $76.2 \mathrm{gmol} / \mathrm{m}^{3}$, which was under the condition of $200^{\circ} \mathrm{C}$ and $60,000 \mathrm{hr}^{-1}$ space velocity. In order to get the correct $\mathrm{NH}_{3}$ storage prediction under this condition or even lower temperatures, the total storage capacity of the model should be higher than this value. In addition, the total storage capacity must be partitioned between the two storage sites. The $\mathrm{NH}_{3}$ adsorption and desorption rates of $\mathrm{R} 1-4$ also affect the $\mathrm{NH}_{3}$ stored on the catalyst. Different combinations of the model parameters including the storage capacity on each site, and the $\mathrm{NH}_{3}$ adsorption and desorption reaction rate constants for each site were used in the simulation to find the best possible fit by comparing the simulation results with the experimental measurements during steps 2-4 for each of the test conditions. Since one reaction rate constant works for one temperature and the TPD test covered a wide temperature range, the TPD data were not used in this process. In this step, the $\mathrm{NH}_{3}$ adsorption reaction was considered as a non-activated reaction with a rate constant independent of temperatures, with a resulting activation energy equal to 0 . 


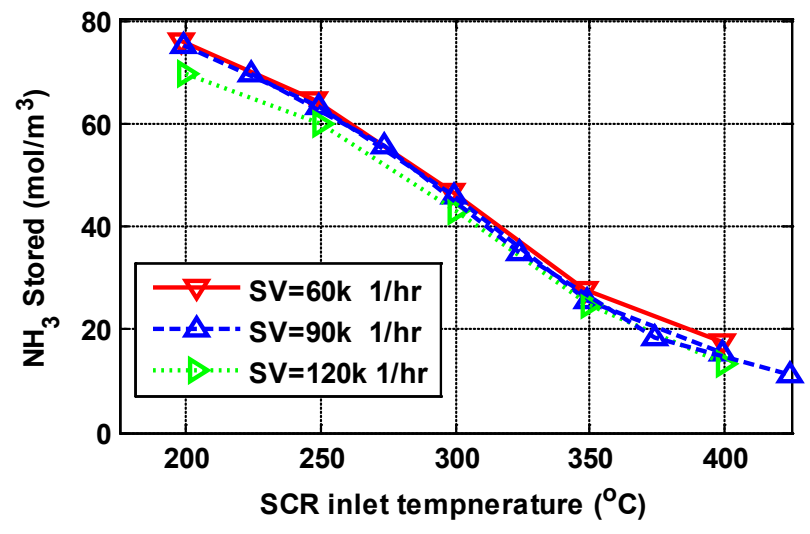

Figure 4.9: $\mathrm{NH}_{3}$ stored on the catalyst at step 2 of the protocol for each reactor test condition

After manual tuning of the model parameters, the optimization method was carried out to further optimize the model parameters using the manually found approximate parameters as a starting point. The optimization method was conducted for steps 2-4 of the test protocol for each test condition. A total of 6 model parameters including the $\mathrm{NH}_{3}$ storage capacity on each of the two sites and $\mathrm{NH}_{3}$ adsorption /desorption reaction rate constants for each of the two sites were identified for every test condition through the procedure presented above.

As discussed above, the initial storage capacity of the model was determined to be higher than $76.2 \mathrm{gmol} / \mathrm{m}^{3}$. For each test condition, the storage capacities and reaction rate constants for the $\mathrm{NH}_{3}$ adsorption/desorption were optimized. The average of the optimal total storage capacities for all test conditions was considered to be the optimal storage capacity of the model. The optimal reaction rate constants for $\mathrm{NH}_{3}$ adsorption and desorption reactions identified for each test condition was plotted in Arrhenius form as shown in Figure 4.10 to find the $A$ and $E$ of the reactions. The slopes and the intercepts of the fit trend lines in Figure 4.10 were used for calculation of $A$ and $E$ for adsorption and desorption reactions on the two sites using Equation 4.23. The standard deviation of the slope of each reaction was used to determine a confidence range for the activation energy of the reaction. The storage parameter identification results are given in Table 4.4. A trend line with the slope of zero was fitted for the $\mathrm{NH}_{3}$ adsorption reaction 
on each storage site to constrain activation energy to be zero. The results show that the activation energy of $\mathrm{NH}_{3}$ desorption on the $2^{\text {nd }}$ site is higher than that on the $1^{\text {st }}$ site. This means that the $\mathrm{NH}_{3}$ stored on the second site needs more energy to be desorbed from the catalyst. The bigger difference between $\mathrm{NH}_{3}$ adsorption and desorption reaction rate constants under lower temperatures explains why the SCR stores more $\mathrm{NH}_{3}$ at lower temperature conditions.

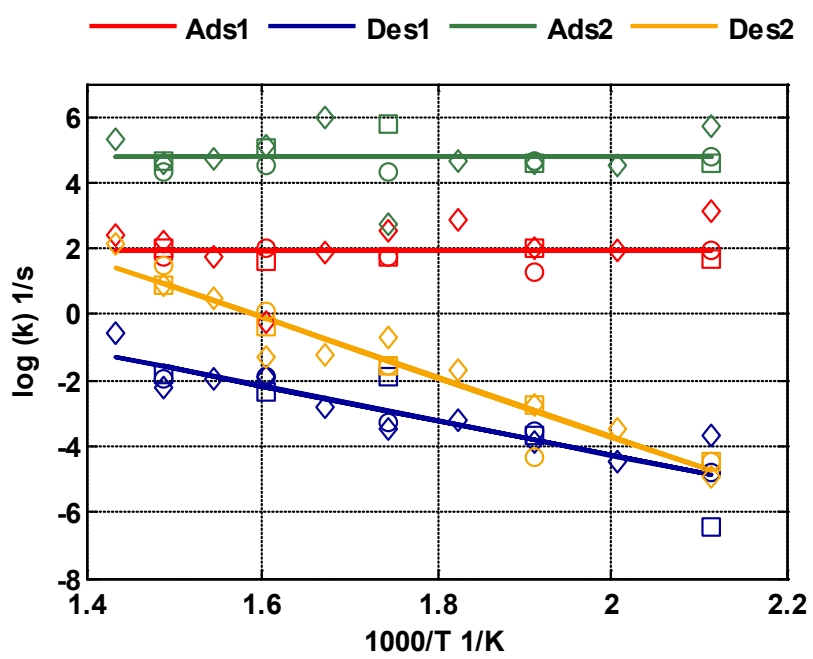

Figure 4.10: Arrhenius plot of reaction rate constants for $\mathrm{NH}_{3}$ adsorption and desorption on two sites (Square: SV = 60,000 hr $^{-1}$, Diamonds: $90,000 \mathrm{hr}^{-1}$, Circle: $120,000 \mathrm{hr}^{-1}$ )

Table 4.4: Identified $\mathrm{NH}_{3}$ storage parameters

\begin{tabular}{|c|c|c|cc|c|}
\hline$\Omega 1$ & \multicolumn{5}{|c|}{$43.0 \mathrm{gmol} / \mathrm{m}^{3}$} \\
\hline$\Omega 2$ & $\mathbf{5}$ & $\mathbf{c}$ & $\mathbf{A}$ & $\mathbf{A}$ \\
\hline & $\mathbf{m}$ & $\mathbf{A J} / \mathbf{g m o l})$ \\
\hline R1: Ads1 & 0 & 1.92 & $6.80 \mathrm{E}+00 \mathrm{~m} / \mathrm{gmol} . \mathrm{s}$ & 0 \\
\hline R2: Des1 & $-5.27 \pm 0.64$ & 6.25 & $5.20 \mathrm{E}+03$ & $1 / \mathrm{s}$ & $52.5 \pm 5.31$ \\
\hline R3: Ads2 & 0 & 4.77 & $1.17 \mathrm{E}+02 \mathrm{~m}^{3} / \mathrm{gmol} . \mathrm{s}$ & 0 \\
\hline R4: Des2 & $-9.12 \pm 0.56$ & 14.5 & $1.97 \mathrm{E}+06$ & $1 / \mathrm{s}$ & $71.6 \pm 4.67$ \\
\hline
\end{tabular}




\subsection{3 $\mathrm{NH}_{3}$ Oxidation Parameters}

Reactor data from step 3 of the protocol was used to calibrate the $\mathrm{NH}_{3}$ oxidation reactions ( $R 5$ and 6 ) which are the major reactions taking place on the $1^{\text {st }}$ site during this step except the adsorption and desorption reactions. The reaction R6 forms NO which reacts with $\mathrm{NH}_{3}$ through standard SCR reactions. The standard SCR reactions at step 3 were not calibrated in this step of the calibration process because of their insignificant effect on the results. Similar to the parameter identification process described in the previous section, a manual tuning of the reaction rate constants was followed by the optimization method. The process was conducted for step 3 of the protocol under each test condition. The optimal reaction rate constants for the $\mathrm{NH}_{3}$ oxidation reactions obtained after the optimization process were plotted in Arrhenius form which is given as Figure 4.11. $A$ and $E$ for each $\mathrm{NH}_{3}$ oxidation reaction were calculated using the slope and intercept trend lines according to Equation 4.23 and are given in Table 4.5. Both $\mathrm{NH}_{3}$ oxidation reactions have relatively low reaction rate constants. The effect of temperature on the $2^{\text {nd }}$ oxidation reaction is more obvious because of its higher activation energy.

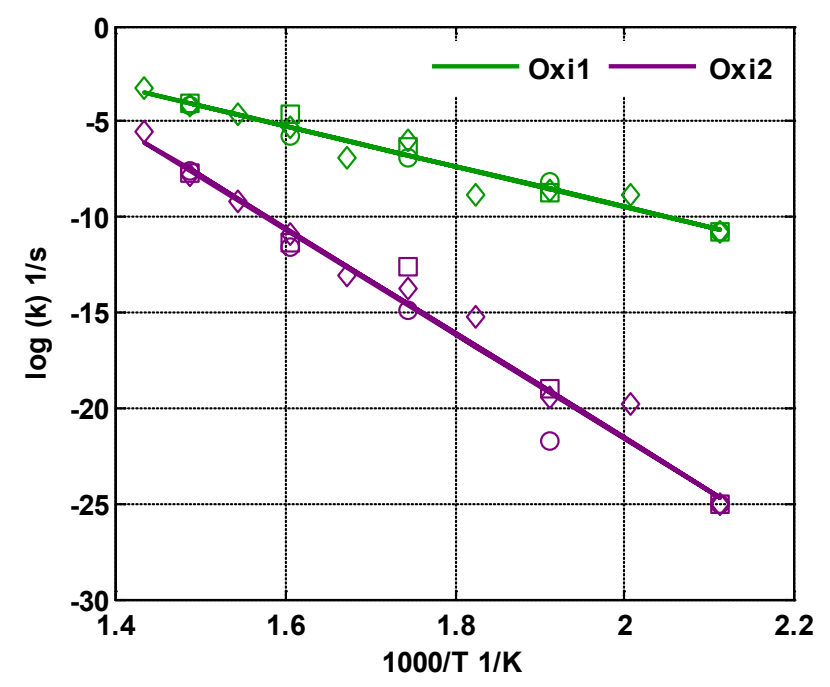

Figure 4.11: Arrhenius plot of reaction rate constants for $\mathrm{NH}_{3}$ oxidation reactions

(Square: SV $=60,000 \mathrm{hr}^{-1}$, Diamonds:90,000 hr-1, Circle: $120,000 \mathrm{hr}^{-1}$ ) 
Table 4.5: Identified $\mathrm{NH}_{3}$ oxidation parameters

\begin{tabular}{|c|c|c|c|c|}
\hline Reaction & $\boldsymbol{m}$ & $\boldsymbol{c}$ & $\boldsymbol{A}(\mathbf{1 / s})$ & $\boldsymbol{E}$ (kJ/gmol) \\
\hline R5: Oxi1 & $-10.5 \pm 0.52$ & 11.5 & $1.02 \mathrm{E}+05$ & $87.3 \pm 4.34$ \\
\hline R6: Oxi2 & $-27.3 \pm 1.08$ & 33.1 & $2.27 \mathrm{E}+14$ & $227 \pm 8.94$ \\
\hline
\end{tabular}

\subsection{4 $\mathrm{NO}+\mathrm{NH}_{3} \mathrm{SCR}$ Reaction Parameters}

With the model parameters identified in the previous sections, the model calibration was then focused on the individual SCR reactions. Although fast SCR reaction (R9) is dominant during $\mathrm{NO}_{x}+\mathrm{NH}_{3}$ SCR steps 5 to 9 of the protocol, other reactions including standard SCR, slow SCR, $\mathrm{NO}$ oxidation, $\mathrm{NH}_{3}$ oxidation, and $\mathrm{N}_{2} \mathrm{O}$ formation also take place because of the presence of both $\mathrm{NO}$ and $\mathrm{NO}_{2}$ in the exhaust stream. Fewer reactions take place during NO+SCR steps 10 to 14 . The major reactions during this segment include $1^{\text {st }}$ and $2^{\text {nd }}$ standard SCR reaction and NO oxidation reaction (R7, 8, and 11). Other reactions taking place in this segment of the protocol includes $\mathrm{NH}_{3}$ adsorption/desorption, $\mathrm{NH}_{3}$ oxidation reactions, and $\mathrm{NO}_{2}$ involved $\mathrm{SCR}$ reactions because of the possible $\mathrm{NO}_{2}$ formation through the $\mathrm{NO}$ oxidation reaction. The determined model parameters for $\mathrm{NH}_{3}$ adsorption/desorption and $\mathrm{NH}_{3}$ oxidation reactions were used but not optimized in this step of the calibration process. The $\mathrm{NO}_{2}$ involved SCR reactions were also not calibrated in this step because of their insignificant effect on the results. The parameter identification process was conducted for steps 10 to 14 of the protocol for all test conditions and the identified optimal reaction rate constants were plotted in Arrhenius form as shown in Figure 4.12 and the $A$ and $E$ of each reaction are given in Table 4.6. The $1^{\text {st }}$ standard SCR reaction has a higher reaction rate constant, meaning that it is the dominant reaction among the three reactions during the $\mathrm{NO}+\mathrm{NH}_{3}$ SCR steps 10 to 14 . 


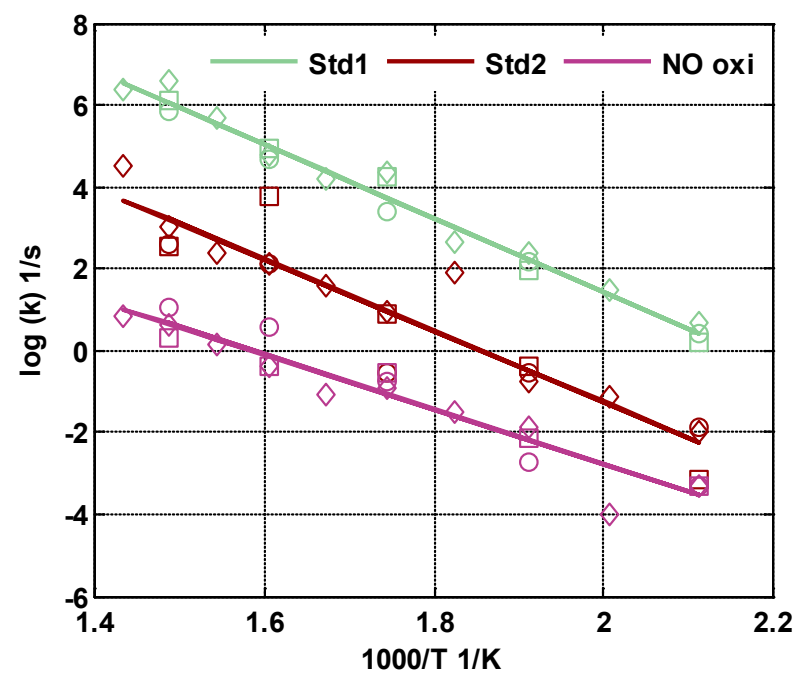

Figure 4.12: Arrhenius plot of reaction rate constants for the two standard SCR reactions and NO oxidation reaction (Square: SV $=60,000 \mathrm{hr}^{-1}$, Diamonds:90,000 hr-1, Circle: $120,000 \mathrm{hr}^{-1}$ )

Table 4.6: Identified model parameters for standard SCR reactions and NO oxidation reaction

\begin{tabular}{|c|c|c|c|c|}
\hline Reaction & $\boldsymbol{m}$ & $\boldsymbol{c}$ & $\boldsymbol{A}$ & $\boldsymbol{E}$ (kJ/gmol) \\
\hline R7: Std1 & $-8.99 \pm 0.31$ & 19.4 & $2.74 \mathrm{E}+08 \mathrm{~m}^{3} / \mathrm{gmol} . \mathrm{s}$ & $74.8 \pm 2.60$ \\
\hline R8: Std2 & $-8.67 \pm 0.77$ & 16.1 & $9.71 \mathrm{E}+06 \mathrm{~m}^{3} / \mathrm{gmol} . \mathrm{s}$ & $72.1 \pm 6.37$ \\
\hline R11: NO Oxi & $-6.69 \pm 0.45$ & 10.6 & $3.99 \mathrm{E}+04 \quad 1 / \mathrm{s}$ & $55.6 \pm 3.75$ \\
\hline
\end{tabular}

\subsection{5 $\mathrm{NO}_{2}+\mathrm{NH}_{3}$ SCR Reaction Parameters}

The reactions taking place during $\mathrm{NO}_{2}+\mathrm{NH}_{3}$ steps 15 to 19 include slow SCR reaction (R10), $\quad \mathrm{N}_{2} \mathrm{O}$ formation reaction (R12), $\quad \mathrm{NO}_{2}$ decomposition reaction (R11), $\mathrm{NH}_{3}$ adsorption/desorption reactions (R 1-4), and the NO involved SCR reaction because of the possible $\mathrm{NO}$ formation through the $\mathrm{NO}_{2}$ decomposition reaction. As discussed in the previous section, the $\mathrm{NO}_{2}$ decomposition reaction is the reversible reaction of the $\mathrm{NO}$ oxidation reaction which was already calibrated as discussed in the previous section, leaving parameters for the slow SCR reaction and the $\mathrm{N}_{2} \mathrm{O}$ formation reaction to identify in this step of the calibration. As discussed in Appendix G, certain parts of the reactor data at low temperatures were not suitable for model calibration purposes. As a result, only high temperature runs were considered in this part of the calibration. After 
conducting the parameter identification process for the two reactions during steps 15 to 19 for the high temperature runs, the identified reaction rate constants were plotted in Arrhenius form shown in Figure 4.13 and the $A$ and $E$ of each reaction are given in Table 4.7.

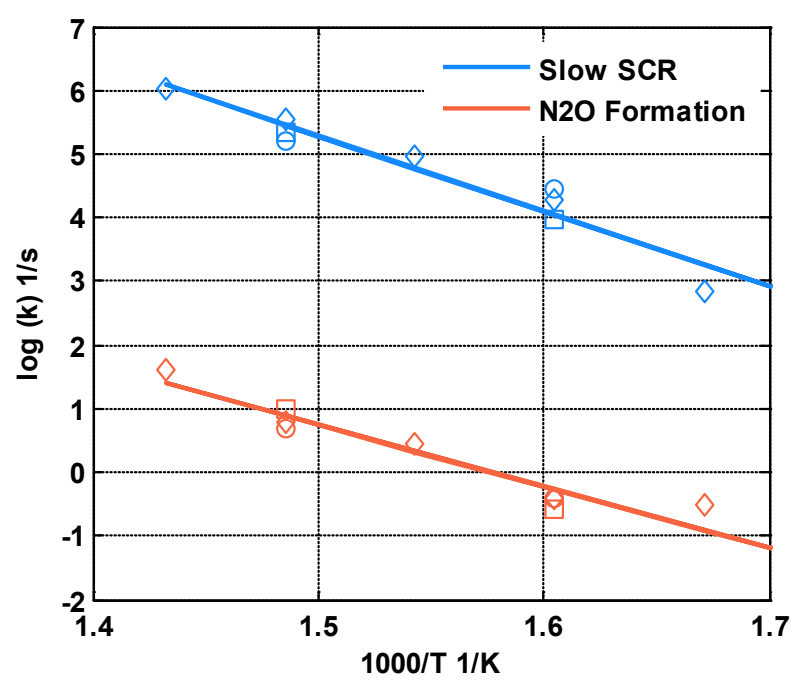

Figure 4.13: Arrhenius plot of reaction rate constants for slow $\mathrm{SCR}$ reactions and $\mathrm{N}_{2} \mathrm{O}$ formation reaction (Square: SV $=60,000 \mathrm{hr}^{-1}$, Diamonds:90,000 $\mathrm{hr}^{-1}$, Circle: 120,000 $\mathrm{hr}^{-1}$ )

Table 4.7: Identified model parameters for slow SCR reactions and $\mathrm{N}_{2} \mathrm{O}$ formation reaction

\begin{tabular}{|c|c|c|c|c|}
\hline Reaction & m & C & A (m ( $^{\text {/gmol.s) }}$ & E (kJ/gmol) \\
\hline R10: Slow SCR & $-11.8 \pm 1.21$ & 22.9 & $9.67 \mathrm{E}+09$ & $98.2 \pm 10.0$ \\
\hline R12: $\mathrm{N}_{2}$ O Form. & $-9.65 \pm 1.09$ & 15.2 & $4.05 \mathrm{E}+06$ & $80.2 \pm 9.13$ \\
\hline
\end{tabular}

\subsection{6 $\mathrm{NO}_{\mathrm{x}}+\mathrm{NH}_{3} \mathrm{SCR}$ Reaction Parameters}

With the parameters identified in previous sections, the calibration of the fast SCR reaction (R9) became the last step. The fast SCR reaction rate constant was optimized using reactor data from step 5 to 9 of the protocol following the same process as in the previous sections for all test conditions except the $200^{\circ} \mathrm{C}$ temperature data as discussed in Appendix G. The optimal reaction rate constant for each test condition was plotted in Arrhenius form as shown in Figure 4.14 and the $A$ and $E$ of the reaction are given in 
Table 4.8. Compared to the other SCR reactions discussed above, the fast SCR reaction has the highest reaction rate constant which means it results in the highest reaction rate under the same temperature conditions.

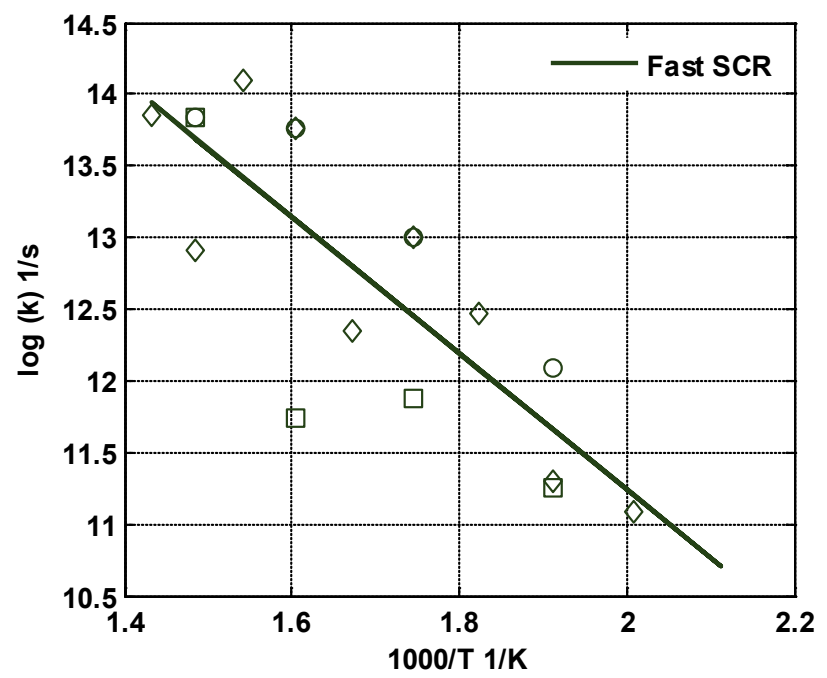

Figure 4.14: Arrhenius plot of reaction rate constants for fast SCR reaction (Square: SV $=60,000 \mathrm{hr}^{-1}$, Diamonds: 90,000 $\mathrm{hr}^{-1}$, Circle: $120,000 \mathrm{hr}^{-1}$ )

Table 4.8: Identified model parameters for fast SCR reaction

\begin{tabular}{|c|c|c|c|c|}
\hline Reaction & $\mathbf{m}$ & C & $\mathrm{A}\left(\mathrm{m}^{6} / \mathrm{gmol}^{2} . \mathrm{s}\right)$ & $E$ (kJ/gmol) \\
\hline R9: Fast SCR & $-4.76 \pm 0.86$ & 20.8 & $1.03 E+09$ & $39.6 \pm 7.13$ \\
\hline
\end{tabular}

The identified model parameters for the model calibration to the reactor experimental data are summarized and shown in Table 4.9. 
Table 4.9: SCR model calibration results based on the reactor data

\begin{tabular}{|c|c|c|c|c|c|}
\hline $\mathbf{\Omega 1}$ & \multicolumn{5}{|c|}{$43.0 \mathrm{gmol} / \mathrm{m}^{3}$} \\
\hline $\mathbf{\Omega 2}$ & \multicolumn{5}{|c|}{$39.3 \mathrm{gmol} / \mathrm{m}^{3}$} \\
\hline Reaction & $m$ & $c$ & & & $E(\mathrm{~kJ} / \mathrm{gmol})$ \\
\hline Ads1 & 0 & 1.92 & $6.80 \mathrm{E}+0$ & $\mathrm{~m}^{3} / \mathrm{gmol} . \mathrm{s}$ & 0 \\
\hline Des1 & $-5.27 \pm 0.64$ & 6.25 & $5.20 E+0$ & $1 / \mathrm{s}$ & $43.8 \pm 5.31$ \\
\hline Ads2 & 0 & 4.77 & $1.17 \mathrm{E}+0$ & $\mathrm{~m}^{3} / \mathrm{gmol} . \mathrm{s}$ & 0 \\
\hline Des2 & $-9.12 \pm 56$ & 14.5 & $1.97 E+06$ & $1 / s$ & $75.7 \pm 4.67$ \\
\hline $\mathrm{NH}_{3} \mathrm{Oxi1}$ & $-10.5 \pm 0.52$ & 11.5 & $1.02 \mathrm{E}+0$ & $1 / s$ & $87.3 \pm 4.34$ \\
\hline $\mathrm{NH}_{3} \mathrm{Oxi2}$ & $-27.3 \pm 1.08$ & 33.1 & $2.27 \mathrm{E}+1$ & $1 / s$ & $227 \pm 8.94$ \\
\hline Std1 & $-8.99 \pm 0.31$ & 19.4 & $2.74 \mathrm{E}+0$ & $\mathrm{~m}^{3} / \mathrm{gmol} . \mathrm{s}$ & $74.8 \pm 2.60$ \\
\hline Std2 & $-8.66 \pm 0.77$ & 16.1 & $9.71 \mathrm{E}+0$ & $\mathrm{~m}^{3} / \mathrm{gmol} . \mathrm{s}$ & $72.0 \pm 6.37$ \\
\hline NO Oxi & $-6.69 \pm 0.45$ & 10.6 & $3.99 E+0$ & $1 / s$ & $55.6 \pm 3.75$ \\
\hline Slow & $-11.8 \pm 1.21$ & 22.9 & $9.67 \mathrm{E}+0$ & $\mathrm{~m}^{3} / \mathrm{gmol} . \mathrm{s}$ & $98.2 \pm 10.0$ \\
\hline $\mathrm{N}_{2} \mathrm{O}$ Form. & $-9.65 \pm 1.10$ & 15.2 & $4.05 E+0$ & $\mathrm{~m}^{3} / \mathrm{gmol} . \mathrm{s}$ & $80.2 \pm 9.13$ \\
\hline Fast & $-4.76 \pm 0.86$ & 20.8 & $1.03 E+0$ & $\mathrm{~m}^{6} / \mathrm{gmol}^{2} . \mathrm{s}$ & $39.5 \pm 7.13$ \\
\hline
\end{tabular}

\subsection{Sample Line Model Development}

As discussed in the previous section, the $\mathrm{NH}_{3}$ slip measurements from the IMR-MS were delayed because of the $\mathrm{NH}_{3}$ adsorption on the sample line wall. Using the delayed $\mathrm{NH}_{3}$ measurements in the SCR model calibration process without correction will cause an error in the model kinetic parameters. As a result, it is important to quantify the $\mathrm{NH}_{3}$ adsorption and desorption process in the sample line and take it into consideration during calibration of the SCR model with the engine data.

A sample line model which simulates the $\mathrm{NH}_{3}$ adsorption and desorption process in the sample line was developed to reduce the effect of the process on the SCR model 
calibration. The model considers the sample line as a $\mathrm{NH}_{3}$ storage site with certain $\mathrm{NH}_{3}$ storage capacity and includes $\mathrm{NH}_{3}$ adsorption and desorption reactions. The storage capacity and the reaction rate constant of each reaction are temperature dependent. Since the sample line was constantly heated to $190^{\circ} \mathrm{C}$, the storage capacity and the reaction rate constants are considered to be constants. As a result, three model parameters including $\mathrm{NH}_{3}$ storage capacity of the sample line and two reaction rate constants for $\mathrm{NH}_{3}$ adsorption and desorption reactions need to be identified by calibrating the model to the experimental data from the sample line tests discussed in the previous section.

As shown in Figure 4.15, the $\mathrm{NH}_{3}$ calibration gas from the gas cylinder with a $\mathrm{NH}_{3}$ concentration of $103.8 \mathrm{ppm}$ was used as a step input to the sample line model. The IMRMS measurement was switched to sampling from the cylinder at 3 minutes to study the $\mathrm{NH}_{3}$ adsorption process in the sample line. After the $\mathrm{NH}_{3}$ measurement from the analyzer reached steady state, the gas sampling was switched to room air at 28 minutes to study the $\mathrm{NH}_{3}$ desorption process in the sample line. The output $\mathrm{NH}_{3}$ concentration of the sample line model was from the measurement of the IMR-MS. The $\mathrm{NH}_{3}$ storage capacity of the sample line was calculated by integrating the difference between the model input and output $\mathrm{NH}_{3}$ concentrations during $\mathrm{NH}_{3}$ adsorption. The reaction rate constants for adsorption and desorption reactions were tuned to match the model simulated outlet $\mathrm{NH}_{3}$ with the $\mathrm{NH}_{3}$ measurements from the IMR-MS. A manual calibration along with an optimization method was conducted to find the optimal model parameters which gave the smallest error between model simulation and experimental measurements. The identified optimal model parameters and the model simulation compared to experimental measurements are shown in Figure 4.15. The storage capacity of the sample line was determined to be $0.12 \mathrm{gmol} / \mathrm{m}^{3}$. The adsorption and desorption reaction rate constants were $3.5 \mathrm{~m}^{3} / \mathrm{gmol}$.s and $0.011 / \mathrm{s}$ respectively.

As can be seen from Figure 4.15, the model can correctly simulate the $\mathrm{NH}_{3}$ measurement delay caused by the $\mathrm{NH}_{3}$ adsorption in the sample line. The model simulation approaches to the steady state faster than the measurement probably because there are other factors affecting the measurement in the analyzer including pre- 
filter effect, slow response of the detector, etc. The $\mathrm{NH}_{3}$ desorption process from the sample line after 28 minutes can also be predicted by the sample line model.

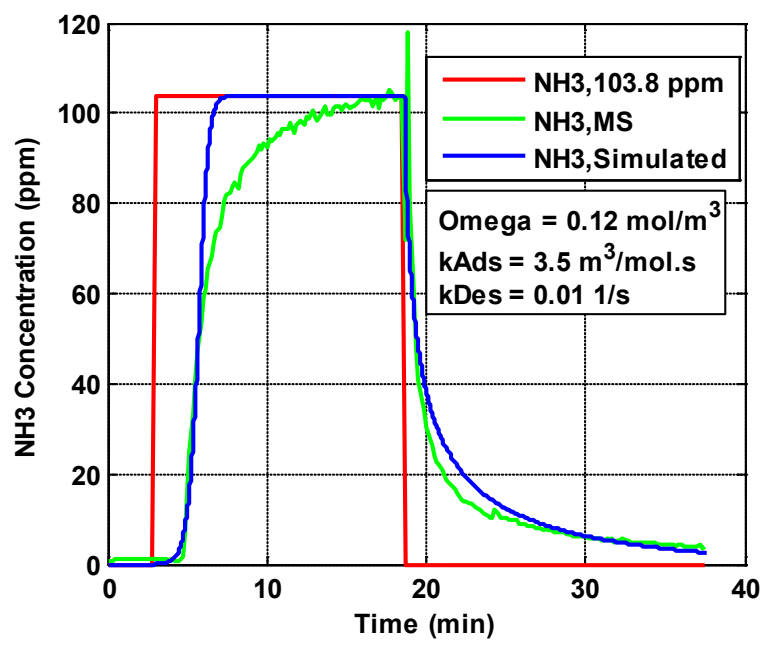

Figure 4.15: Sample line model predicted $\mathrm{NH}_{3}$ compared to the IMR-MS measure during $103.8 \mathrm{ppm} \mathrm{NH}_{3}$ calibration gas as a step input

The model performance was validated using another set of sample line experimental data from the SCR engine experiment as shown in Figure 4.16. The $\mathrm{NH}_{3}$ measurement from the $\mathrm{NH}_{3}$ sensor was considered to be real-time and used as the input to the sample line model. The $\mathrm{NH}_{3}$ measurements from the IMR-MS were used as the output to the model. The model predicted $\mathrm{NH}_{3}$ at the outlet of the sample line was compared to the IMR-MS measurements as shown in Figure 4.16. The results show good agreement between the model prediction and experimental measurements in terms of measurement delay and the transient adsorption/desorption process. The sample line model was used to post process the SCR model predicted $\mathrm{NH}_{3}$ to take into account the effect of the $\mathrm{NH}_{3}$ measurement delay. 


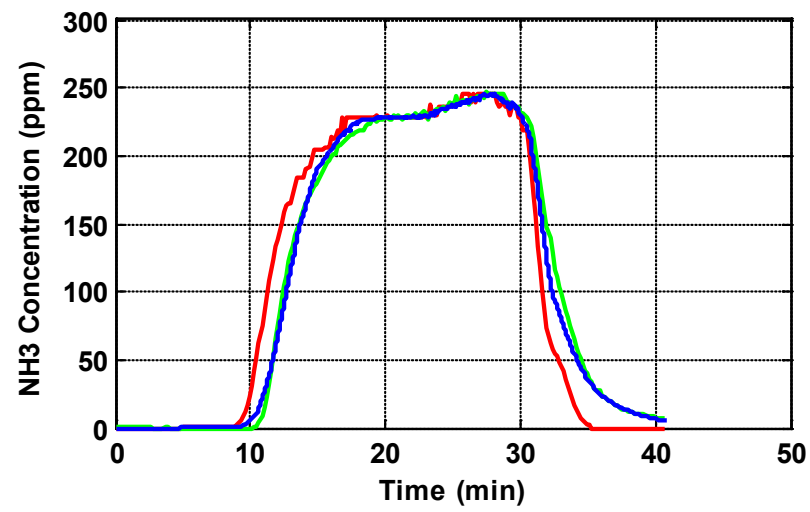

Figure 4.16: Sample line model predicted $\mathrm{NH}_{3}$ compared to the IMR- MS measured $\mathrm{NH}_{3}$ using $\mathrm{NH}_{3}$ sensor measurement during engine test as the input (Red: $\mathrm{NH}_{3}$ sensor measurement, Green: IMR-MS measurement, Blue: Sample line model predictions)

\subsection{SCR Model Calibration with Engine Data}

The main focus of the SCR model calibration was to determine the optimal set of model parameters which gave the best agreement between the model simulation and the engine experimental measurements. The model parameters that need to be determined include $A$ and $E$ for the SCR reactions and the two storage capacities for the two storage sites. The model parameters from the SCR model calibrated to the reactor data were used as a starting calibration for the engine data based SCR model. A manual tuning of the parameters along with an optimization method was performed during the SCR engine data based model calibration process.

The model was first run by manually varying the individual parameters to determine how the change in each parameter affects the simulation results. This determined the response of tuning each model parameter. Iterations of this procedure were performed to find the approximate set of parameters for each experimental run. This provided an initial set that were used as the starting parameters for the optimization method. The optimization method works by varying the parameters of the model to make predictions with the smallest error compared to experimental results. Similarly to the optimization process for calibration of the model with the reactor data, the error between simulation 
results and experimental measurements were defined as the cost values that can be minimized by the MATLAB "fmincon" function. This procedure was also performed by optimizing different model parameters for each experimental run. A smaller weighing factor was used for $\mathrm{NH}_{3}$ when calculating the cost function value because measurement delay was known to be associated with the $\mathrm{NH}_{3}$ measurements from the IMR-MS. The model simulated $\mathrm{NH}_{3}$ will be post processed by a sample line model which simulates the $\mathrm{NH}_{3}$ measurement delay caused by $\mathrm{NH}_{3}$ adsorption and desorption in the sample line. The sample line model introduced in the previous section was incorporated into the SCR model simulation to eliminate the $\mathrm{NH}_{3}$ measurement delay effects. This manual calibration combined with optimization process was performed for each set of experimental data for iterations to achieve optimal match between simulation and experimental results.

The $\mathrm{N}_{2} \mathrm{O}$ formation, $\mathrm{NO}$ oxidation, and the $2^{\text {nd }} \mathrm{NH}_{3}$ oxidation reactions were not calibrated for the engine data based SCR model. Because of the interference issues between $\mathrm{N}_{2} \mathrm{O}$ and interferent $\mathrm{CO}_{2}$ which is present in the exhaust. The low levels of $\mathrm{N}_{2} \mathrm{O}$ (< 100 ppm) concentrations cannot be accurately measured by the IMR-MS. The parameters for the $\mathrm{N}_{2} \mathrm{O}$ formation reaction were retained from the reactor calibration. The effect of temperature on the $\mathrm{NO}$ oxidation and the $2^{\text {nd }} \mathrm{NH}_{3}$ oxidation reaction rate was not captured because of the measurement tolerance of the analyzer was not small enough to identify the reactions in the complex reaction mechanism.

The identified optimal model parameters for each run are given in Table 4.10. The reaction rate constants for each reaction were then plotted in Arrhenius form as shown in Figure 4.17. Run No. $1^{\prime}$ and $4^{\prime}$ are repeats of run 1 and 4 . It can be observed from Figure 4.17 that the reaction rate constant for each reaction followed a linear trend in the Arrhenius form, meaning that the effect of the temperature on the reaction rates was well captured by the model. The slope $m$ and the interception $c$ of each fit trend line were used to calculate the pre-exponential constant and the activation energy of each reaction. The average of the determined $\mathrm{NH}_{3}$ storage capacity for each site for all runs was considered to be the optimized storage capacity on each site. 


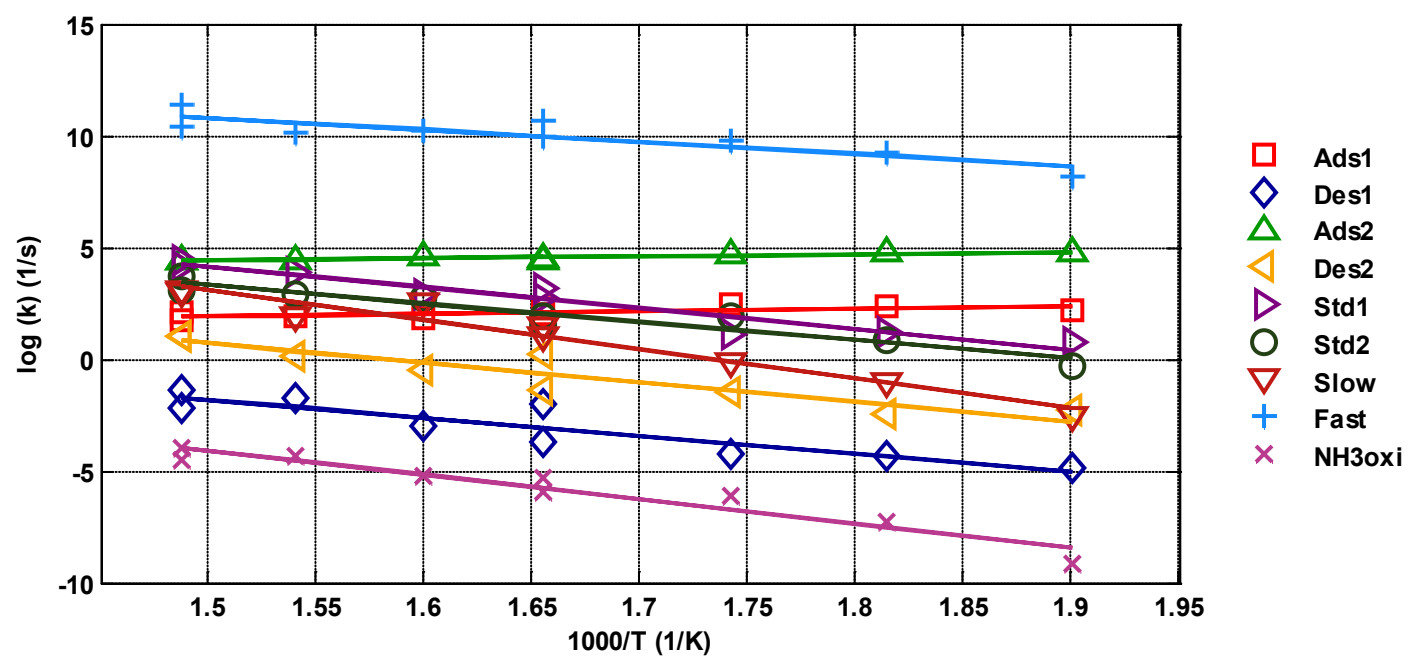

Figure 4.17: Arrhenius plots of reaction rate constant for each reaction in the SCR model based on the engine data 


\begin{tabular}{|c|c|c|c|c|c|c|c|c|c|}
\hline $\begin{array}{r}(s \cdot \text { joub } / \varepsilon w) \\
\text { ozuY :Lレע }\end{array}$ & $\begin{array}{l}8 \\
\\
0\end{array}$ & $\begin{array}{l}8 \\
0\end{array}$ & $\begin{array}{l}8 \\
8 \\
0\end{array}$ & $\begin{array}{l}8 \\
8 \\
0\end{array}$ & $\begin{array}{l}8 \\
8 \\
0\end{array}$ & $\begin{array}{l}8 \\
8 \\
0\end{array}$ & \& & $\begin{array}{l}8 \\
\circ\end{array}$ & ᄋ \\
\hline $\begin{array}{r}(s / L) \\
\text { !xoou }^{-} \text {y :0ly }\end{array}$ & $\begin{array}{l}0 \\
\text { டீ } \\
0\end{array}$ & 8 & $\begin{array}{l}8 \\
8 \\
0\end{array}$ & $\begin{array}{l}8 \\
8 \\
0\end{array}$ & 8: & 8: & 웅 & مै & \& \\
\hline $\begin{array}{r}(s / L) \\
\text { !X0 }{ }^{\varepsilon} \mathrm{HN}^{-}{ }^{-Y}: \mathrm{S}\end{array}$ & 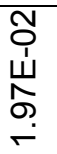 & $\begin{array}{l}\text { Oे } \\
\text { ய் } \\
\text { О } \\
\text { হ }\end{array}$ & 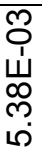 & 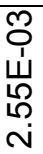 & $\begin{array}{l}\text { m } \\
\text { ய் } \\
\text { ஸ் } \\
\text { ஸ் }\end{array}$ & 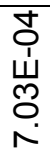 & 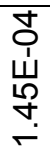 & 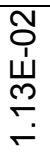 & 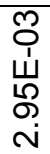 \\
\hline 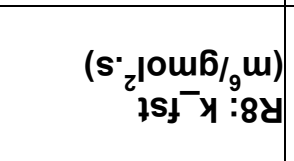 & $\begin{array}{l}\text { ठ } \\
+ \\
\dot{W} \\
\text { Ø } \\
\infty\end{array}$ & 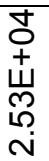 & 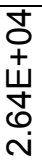 & 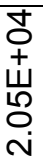 & $\begin{array}{l}\text { ठ } \\
\stackrel{+}{ } \\
\text { ه্ } \\
\text { }\end{array}$ & 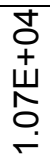 & 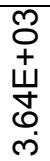 & \begin{tabular}{l}
$\overrightarrow{0}$ \\
+ \\
$\Psi$ \\
\multicolumn{1}{c}{} \\
ம்
\end{tabular} & 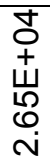 \\
\hline 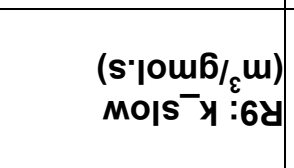 & 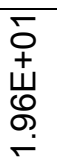 & $\begin{array}{l}\text { ○ } \\
+ \\
\text { W } \\
\text { o } \\
\dot{0}\end{array}$ & $\begin{array}{l}\text { o } \\
+ \\
\stackrel{+}{w} \\
\stackrel{N}{-}\end{array}$ & $\begin{array}{l}\stackrel{8}{\circ} \\
+ \\
\stackrel{+}{\sim} \\
\infty \\
\stackrel{\infty}{N}\end{array}$ & $\begin{array}{l}\check{D} \\
\dot{+} \\
\dot{\sigma} \\
\dot{\sigma}\end{array}$ & 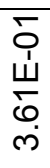 & $\begin{array}{l}\text { Ō } \\
\stackrel{1}{ } \\
\stackrel{N}{\infty} \\
\end{array}$ & $\begin{array}{l}\overline{+} \\
+ \\
\text { W } \\
\infty \\
\text { m. }\end{array}$ & 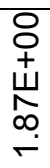 \\
\hline 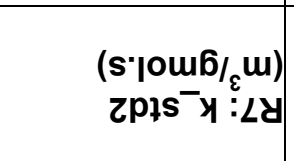 & 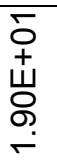 & 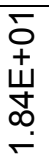 & 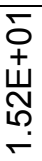 & $\begin{array}{l}\text { Oे } \\
+ \\
\stackrel{+}{\circ} \\
\text { ه } \\
\dot{+}\end{array}$ & 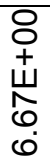 & 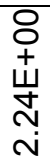 & 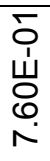 & 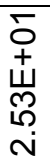 & 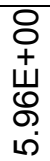 \\
\hline 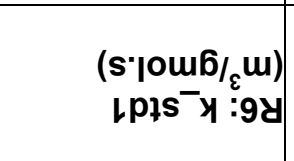 & 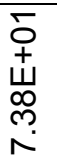 & $\begin{array}{l}\check{+} \\
+ \\
\stackrel{+}{ } \\
\text { ه़ } \\
\dot{\forall}\end{array}$ & 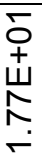 & 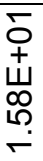 & $\begin{array}{l}8 \\
+ \\
\dot{+} \\
\infty \\
\infty \\
\text { ம் }\end{array}$ & 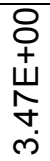 & $\begin{array}{l}\text { ○ } \\
\stackrel{+}{ } \\
\text { யे } \\
\stackrel{0}{ }\end{array}$ & 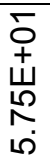 & $\begin{array}{l}\check{0} \\
+ \\
\dot{+} \\
\infty \\
\text { mִ } \\
\Gamma\end{array}$ \\
\hline $\begin{array}{r}(s / L) \\
z s ә p-x: t y\end{array}$ & 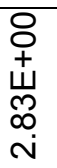 & $\begin{array}{l}8 \\
\circ \\
+ \\
\dot{+} \\
\infty \\
\check{\leftarrow}\end{array}$ & 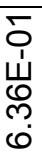 & 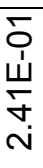 & $\begin{array}{l}\text { ○े } \\
\text { ய் } \\
\text { } \\
\text { ஸे }\end{array}$ & 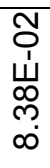 & 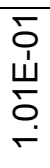 & 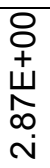 & 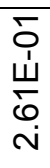 \\
\hline $\begin{array}{l}\left(s^{*} \mid \mathrm{ouw}^{-} / \varepsilon \mathrm{u}\right) \\
z \operatorname{spe}^{-} y: \varepsilon y\end{array}$ & $\begin{array}{l}\bar{o} \\
+ \\
\dot{+} \\
\tilde{m} \\
\infty\end{array}$ & $\begin{array}{l}\overline{0} \\
+ \\
\Psi \\
\varpi \\
\infty\end{array}$ & $\begin{array}{l}\bar{\delta} \\
+ \\
\dot{+} \\
\sigma_{0} \\
\dot{\sigma}\end{array}$ & $\begin{array}{l}\bar{\delta} \\
+ \\
\dot{+} \\
\text { के } \\
\text { '. }\end{array}$ & 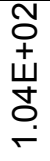 & $\begin{array}{l}\widetilde{D} \\
+ \\
+ \\
\infty \\
\stackrel{+}{-}\end{array}$ & 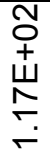 & $\begin{array}{l}\bar{\circ} \\
+ \\
\dot{+} \\
\check{\leftarrow} \\
\infty\end{array}$ & $\begin{array}{l}\overline{0} \\
+ \\
\text { யे } \\
\text { ه } \\
\text { の. }\end{array}$ \\
\hline $\begin{array}{r}(s / L) \\
\text { Lsəp-y :Zy }\end{array}$ & 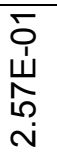 & 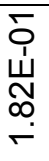 & 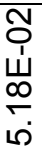 & 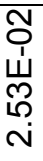 & 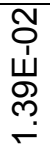 & 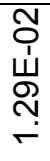 & 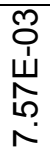 & 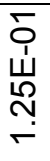 & 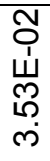 \\
\hline 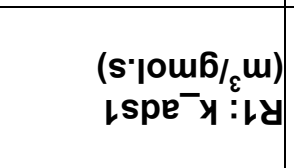 & $\begin{array}{l}\text { ○ } \\
+ \\
\text { 山े } \\
\text { ه } \\
\text { ம் }\end{array}$ & \begin{tabular}{l}
8 \\
+ \\
+ \\
\multirow{+}{*}{} \\
$\omega$ \\
0
\end{tabular} & 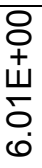 & $\begin{array}{l}8 \\
+ \\
+ \\
\\
\\
\varnothing \\
\infty\end{array}$ & 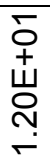 & 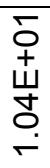 & 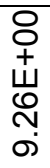 & $\begin{array}{l}8 \\
+ \\
+ \\
\stackrel{+}{\sim} \\
\stackrel{\infty}{ }\end{array}$ & $\begin{array}{l}\text { ○ } \\
+ \\
\text { யे } \\
\text { ○े }\end{array}$ \\
\hline 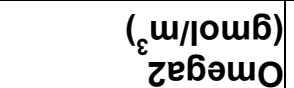 & 离 & ஓं & $\infty$ & $\frac{N}{m}$ & $\hat{e}$ & $\stackrel{m}{m}$ & के & กิ & $\infty$ \\
\hline 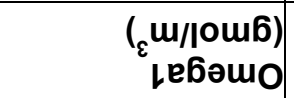 & $\begin{array}{l}\infty \\
0 \\
0 \\
\text { \& }\end{array}$ & 舟 & $\begin{array}{l}+ \\
\dot{\forall}\end{array}$ & 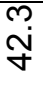 & $\begin{array}{l}\infty \\
L^{\infty}\end{array}$ & 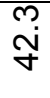 & 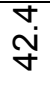 & $\frac{⿱ 亠}{\dot{\sigma}}$ & pi \\
\hline (כ ) ә.пłеләduә & প্ল & $\frac{\varphi}{m}$ & స్ల్ & m & চ্口 & $\stackrel{\infty}{\stackrel{\infty}{\sim}}$ & న్ & ষ্ণে & ల్లి \\
\hline 'ON uny & \ulcorner & $N$ & $m$ & $\forall$ & 10 & 0 & $\curvearrowright$ & $=$ & $\dot{\gamma}$ \\
\hline
\end{tabular}


The calibration results are given in Table 4.11. The standard errors in the slopes of fitted lines were used to calculate the confidence intervals for the activation energy of each reaction. As discussed, the $\mathrm{NH}_{3}$ adsorption reaction was normally considered as a nonactivated reaction with the activation energy of zero in the literature [18]. Efforts were performed during the model calibration process to get the best fit while constraining the activation energy for the $\mathrm{NH}_{3}$ adsorption reactions to be zero. However, it was not successful and the activation energy for the $\mathrm{NH}_{3}$ adsorption reaction was determined to be negative for the SCR model. This was because the model was calibrated with engine data which accounts for the complex urea decomposition process, nonuniformity of the $\mathrm{NH}_{3}$ distribution in the engine exhaust system.

Table 4.11: SCR model calibration results based on the engine data

\begin{tabular}{|c|r|r|rr|r|}
\hline$\Omega 1$ & \multicolumn{5}{|c|}{$43.6 \mathrm{gmol} / \mathrm{m}^{3}$} \\
\hline$\Omega 2$ & \multicolumn{5}{|c|}{$36.0 \mathrm{gmol} / \mathrm{m}^{3}$} \\
\hline Reaction & $\boldsymbol{m}$ & $\boldsymbol{c}$ & \multicolumn{2}{|c|}{$\boldsymbol{A}$} & $\boldsymbol{E}$ (kJ/gmol) \\
\hline Ads1 & $1.22 \pm 0.49$ & 0.49 & $1.08 \mathrm{E}+00$ & $\mathrm{~m}^{3} / \mathrm{gmol} . \mathrm{s}$ & $-10.2 \pm 4.04$ \\
\hline Des1 & $-8.12 \pm 1.45$ & 1.45 & $3.22 \mathrm{E}+04$ & $1 / \mathrm{s}$ & $67.5 \pm 12.1$ \\
\hline Ads2 & $0.91 \pm 0.14$ & 0.14 & $2.11 \mathrm{E}+01$ & $\mathrm{~m}^{3} / \mathrm{gmol} . \mathrm{s}$ & $-7.60 \pm 1.12$ \\
\hline Des2 & $-8.71 \pm 1.31$ & 1.31 & $9.58 \mathrm{E}+05$ & $1 / \mathrm{s}$ & $72.4 \pm 10.9$ \\
\hline Std1 & $-9.29 \pm 0.95$ & 0.95 & $7.18 \mathrm{E}+07$ & $\mathrm{~m}^{3} / \mathrm{gmol} . \mathrm{s}$ & $77.3 \pm 7.92$ \\
\hline Std2 & $-8.22 \pm 0.88$ & 0.88 & $6.17 \mathrm{E}+06$ & $\mathrm{~m}^{3} / \mathrm{gmol}^{3}$ & $68.4 \pm 7.28$ \\
\hline Slow & $-13.1 \pm 1.11$ & 1.11 & $7.13 \mathrm{E}+09$ & $\mathrm{~m}^{3} / \mathrm{gmol}^{.}$ & $109 \pm 9.21$ \\
\hline Fast & $-5.44 \pm 1.15$ & 1.15 & $1.76 \mathrm{E}+08$ & $\mathrm{~m}^{6} / \mathrm{gmol}^{2} . \mathrm{s}$ & $45.2 \pm 9.55$ \\
\hline $\mathrm{NH}_{3}$ Oxi. & $-11.0 \pm 1.10$ & 1.10 & $2.33 \mathrm{E}+05$ & $1 / \mathrm{s}$ & $91.1 \pm 9.18$ \\
\hline
\end{tabular}




\section{Chapter 5.}

\section{Results and Discussion}

The high fidelity SCR model calibration results developed from the reactor and engine experimental data and the applications of the model are discussed in this chapter. The model parameters between the calibrations developed from the reactor and engine data are compared. Differences in the model parameters were determined and the reasons causing the differences were investigated and are discussed. The $\mathrm{NH}_{3}$ maldistribution at the SCR inlet during engine tests was quantified through engine experiments. The effects of the SCR inlet $\mathrm{NO}_{2} / \mathrm{NO}_{x}$ ratio on the $\mathrm{NO}_{x}$ reduction efficiency and $\mathrm{NH}_{3}$ slip under transient conditions was studied using the surrogate HD-FTP cycle. The effects of the $\mathrm{NH}_{3}$ maldistribution on the SCR performance as well as on the kinetic parameters were studied through simulation approach using the calibrated high fidelity SCR model. The hydrothermal aging effects on the SCR performance as well as on the kinetic parameters are also discussed in this chapter.

\subsection{Comparison of Model Parameters}

The comparison of the model parameters identified based on reactor and engine experimental data is shown in Table 5.1. It can be seen that the storage capacities, $\Omega_{1}$ and $\Omega_{2}$ from both calibrations are comparable. There are differences in the kinetic parameters including the pre-exponential factors and activation energies for different SCR reactions. 
Table 5.1: SCR model parameters of the calibrated SCR model along with literature values

\begin{tabular}{|c|c|c|c|c|}
\hline Parameter & $\begin{array}{c}\text { Calibration to } \\
\text { reactor data }\end{array}$ & $\begin{array}{c}\text { Calibration to } \\
\text { engine data }\end{array}$ & $\begin{array}{c}\text { References } \\
{[18,43,129,130]}\end{array}$ & Unit \\
\hline$\Omega 1$ & $4.30 \mathrm{E}+01$ & $4.36 E+01$ & $1.20 \mathrm{E}+02$ & $\mathrm{gmol} / \mathrm{m}^{3}$ \\
\hline$\Omega 2$ & $3.93 E+01$ & $3.60 E+01$ & - & $\mathrm{gmol} / \mathrm{m}^{3}$ \\
\hline A_ads1 & $6.80 E+00$ & $1.08 E+00$ & - & $\mathrm{m}^{3} / \mathrm{gmol} \cdot \mathrm{s}$ \\
\hline E_ads1 & 0 & $-10.2 \pm 4.04$ & 0 & kJ/gmol \\
\hline A_des1 & $5.20 E+02$ & $3.22 E+04$ & - & $1 / \mathrm{s}$ \\
\hline E_des1 & $43.8 \pm 5.31$ & $67.5 \pm 12.1$ & $96.1,97.5$ & $\mathrm{~kJ} / \mathrm{gmol}$ \\
\hline A_ads2 & $1.17 E+02$ & $2.11 E+01$ & - & $\mathrm{m}^{3} / \mathrm{gmol} \cdot \mathrm{s}$ \\
\hline E_ads2 & 0 & $-7.60 \pm 1.12$ & - & $\mathrm{kJ} / \mathrm{gmol}$ \\
\hline A_des2 & $1.97 E+06$ & $9.58 E+05$ & - & $1 / \mathrm{s}$ \\
\hline E_des2 & $75.7 \pm 4.67$ & $72.4 \pm 10.9$ & - & $\mathrm{kJ} / \mathrm{gmol}$ \\
\hline A_nh3oxi1 & $1.02 \mathrm{E}+05$ & $2.33 E+05$ & - & $1 / s$ \\
\hline E_nh3oxi1 & $87.3 \pm 4.34$ & $91.1 \pm 9.18$ & $177,63.8$ & $\mathrm{~kJ} / \mathrm{gmol}$ \\
\hline A_nh3oxi2 & $2.27 E+14$ & - & - & $1 / s$ \\
\hline E_nh3oxi2 & $227 \pm 8.94$ & - & - & kJ/gmol \\
\hline A_std & $2.74 E+08$ & $7.18 \mathrm{E}+07$ & - & $\mathrm{m}^{3} / \mathrm{gmol} \cdot \mathrm{s}$ \\
\hline E_std & $74.8 \pm 2.60$ & $77.3 \pm 7.92$ & $48.7,88.0,89.1$ & $\mathrm{~kJ} / \mathrm{gmol}$ \\
\hline A_std2 & $9.71 E+06$ & $6.17 E+06$ & - & $\mathrm{m}^{3} / \mathrm{gmol} \cdot \mathrm{s}$ \\
\hline E_std2 & $72.1 \pm 6.37$ & $68.4 \pm 7.28$ & - & $\mathrm{kJ} / \mathrm{gmol}$ \\
\hline A_nooxi & $3.99 E+04$ & - & - & $1 / \mathrm{s}$ \\
\hline E_nooxi & $55.6 \pm 3.75$ & - & $23.4 \pm 1.80,-31.6,56$ & $\mathrm{~kJ} / \mathrm{gmol}$ \\
\hline A_slo & $9.67 E+09$ & $7.13 E+09$ & - & $\mathrm{m}^{3} / \mathrm{gmol} \cdot \mathrm{s}$ \\
\hline E_slo & $98.2 \pm 10.0$ & $109 \pm 9.21$ & $58.3,136.3$ & $\mathrm{~kJ} / \mathrm{gmol}$ \\
\hline A_n2o & $4.05 E+06$ & - & - & $\mathrm{m}^{3} / \mathrm{gmol} . \mathrm{s}$ \\
\hline E_n2o & $80.2 \pm 9.13$ & - & $99.8 \pm 16.5,48.2$ & $\mathrm{~kJ} / \mathrm{gmol}$ \\
\hline A_fst & $1.03 E+09$ & $1.76 E+08$ & - & $\mathrm{m}^{6} / \mathrm{gmol}^{2} \cdot \mathrm{s}$ \\
\hline E_fst & $39.6 \pm 7.13$ & $45.2 \pm 9.55$ & $113,32.1,77.1$ & kJ/gmol \\
\hline
\end{tabular}


The major kinetic parameters for the SCR reactions from both models calibrated to the reactor and engine data are compared in Arrhenius form as shown in Figures 5.1-5.4.

It can be seen from Figures 5.1 and 5.2 that flat trend lines were fitted for the $\mathrm{NH}_{3}$ adsorption reactions on both storage sites of the model calibrated to the reactor data, meaning that the reaction is non-activated with activation energy of zero. Negative activation energies were determined for the adsorption reactions when calibrating to the engine data to achieve the lowest cost function values. Differences in the desorption reaction can be seen in Figure 5.1. The desorption reaction on site 1 calibrated to the engine data has lower reaction rate constants and is more affected by the changes in temperature. The desorption reaction on site 2 are similar for the calibrations to the reactor and engine data.

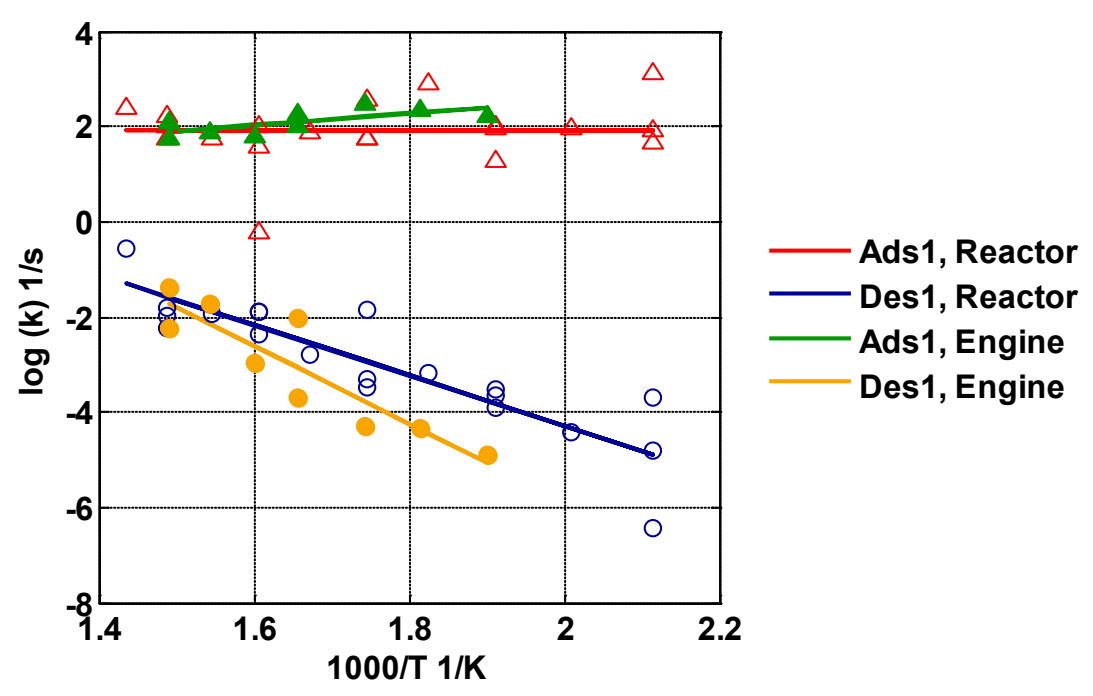

Figure 5.1: Comparison of the kinetic parameters for adsorption and desorption reactions on site 1 


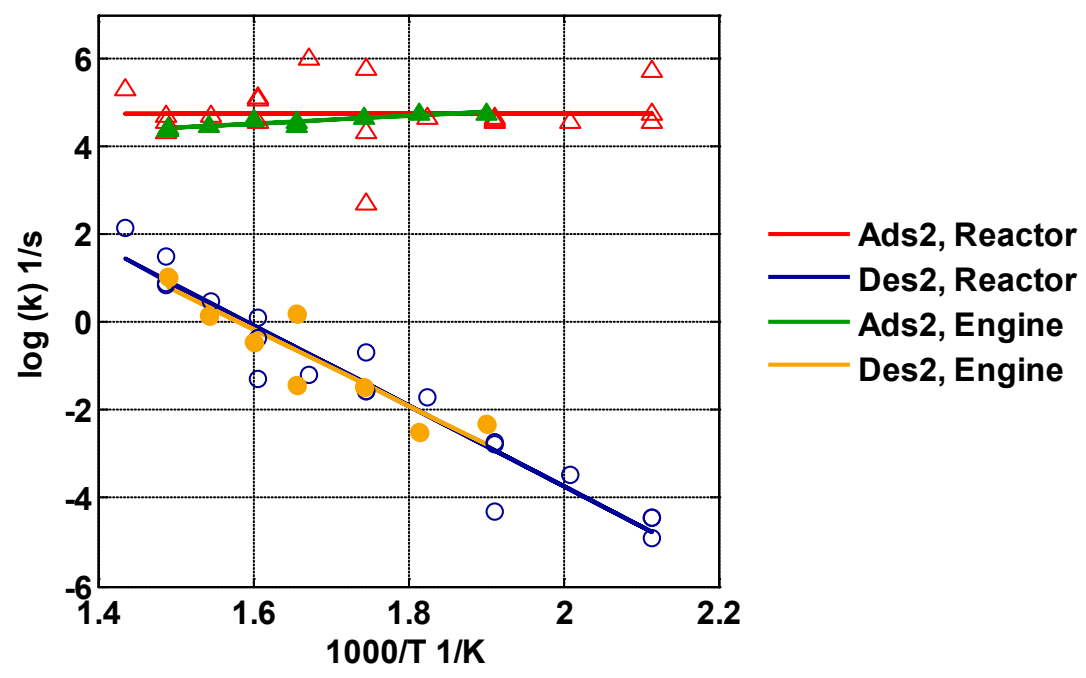

Figure 5.2: Comparison of the kinetic parameters for adsorption and desorption reactions on site 2

The comparison of the standard SCR reaction parameters between the two calibrations is shown in Figure 5.3. It can be seen that the reaction rate constants of the $2^{\text {nd }}$ standard SCR reaction are lower than the $1^{\text {st }}$ standard SCR reaction for both calibrations. Similar slopes of the trend lines indicate that the activation energies are similar for the reactions. The reaction rate constant of the $1^{\text {st }}$ standard SCR reaction calibrated to the engine data is lower that than that calibrated to the reactor data. The reaction rate constants of the $2^{\text {nd }}$ standard SCR reaction calibrated to the engine data are slightly higher that the same reaction calibrated to the reactor data. 


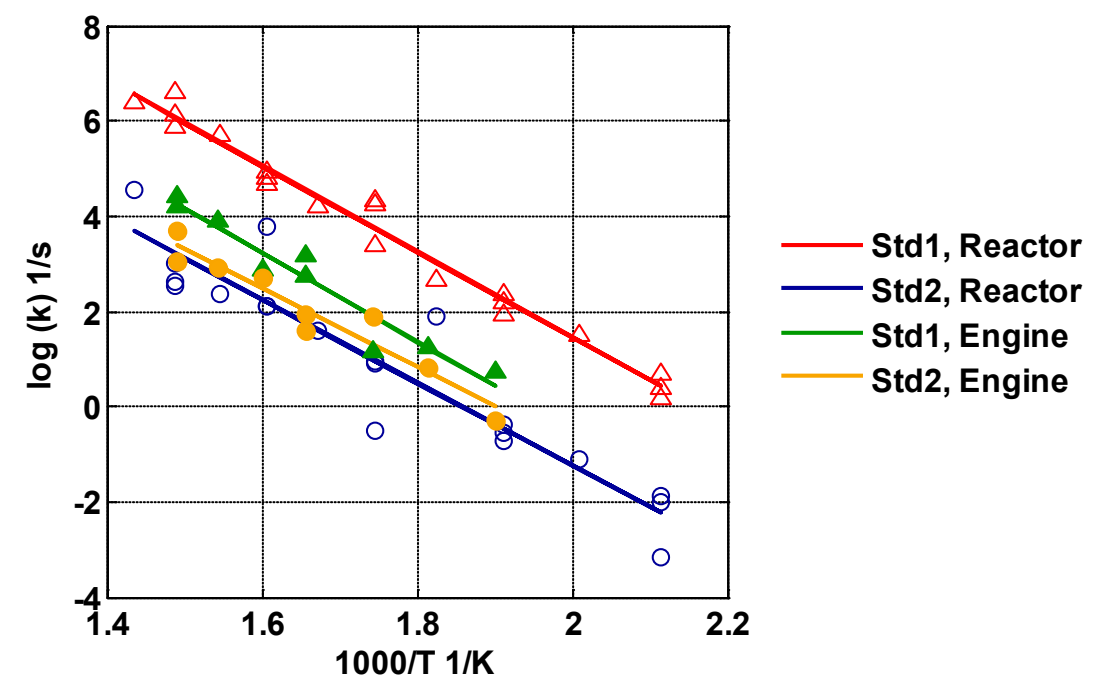

Figure 5.3: Comparison of the kinetic parameters for the standard SCR reactions

The comparisons of the kinetic parameters for the slow and fast SCR reactions between the two calibrations are shown in Figure 5.4. It can be seen that the highest reaction rate constants are associated with the fast SCR reaction. The slopes of the trendlines for the fast SCR reaction calibrated to the reactor and engine data are similar, meaning that similar activation energies were obtained for the two calibrations. The fast SCR reaction calibrated to the engine data has lower reaction rate constants as compared to the reactor data. The same trends are also valid for the slow SCR reaction calibrated to the reactor and engine data. 


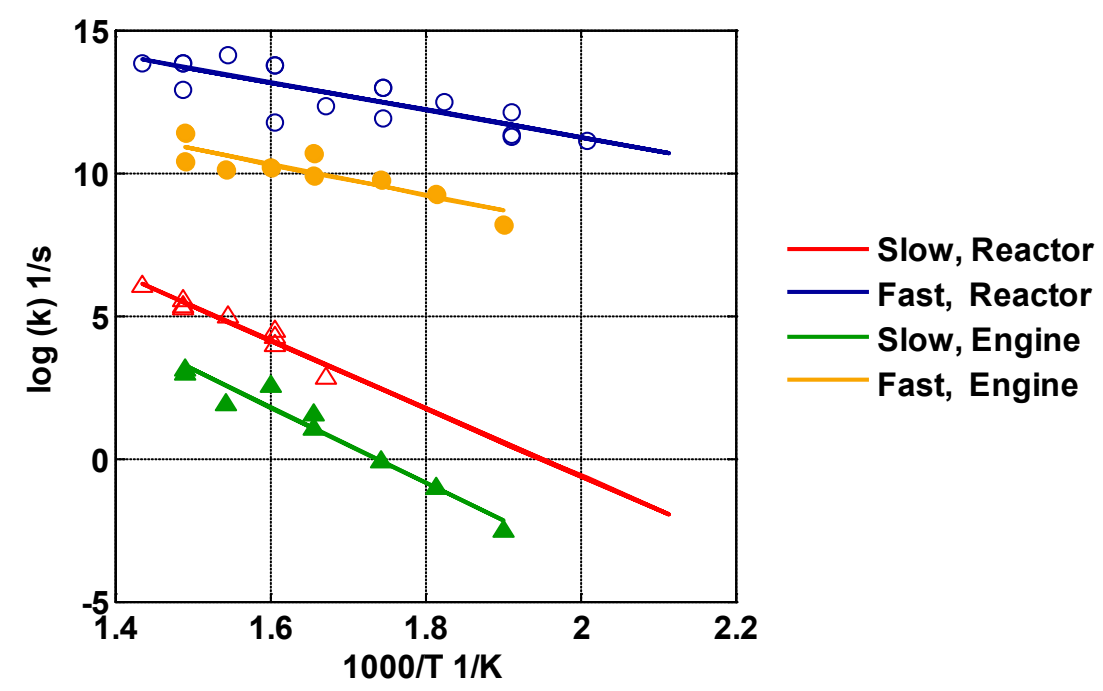

Figure 5.4: Comparison of the kinetic parameters for the fast and slow SCR reactions

Since the catalyst formulations tested in the reactor and engine test cell were the same, it was considered that the more complex engine testing environment caused the differences. The main differences between the reactor and engine tests were (1) complex urea decomposition process, (2) maldistribution of $\mathrm{NH}_{3}$ going into the SCR. Of these differences, maldistribution was postulated to have the largest effect on the calibration. The $\mathrm{NH}_{3}$ maldistribution effects will be discussed later in Section 5.4.

\subsection{SCR Model Calibrated to Reactor Data}

With identified storage capacities and kinetic parameters for each reaction, the model is able to capture the effect of temperature on reaction rates. Additionally, since the model included mass transfer and was calibrated against the experimental data of three different space velocities, the effect of space velocities on SCR performance can be predicted. The parameters identified from the reactor data are given in Table 5.1 along with the calibration based on the engine experimental data and the model parameters from the literature for Cu-zeolite SCR $[18,43,129,130]$. 


\subsubsection{Model Simulation of TPD Experiments}

Three sets of the $\mathrm{NH}_{3}$ TPD experimental data were used for validation of the storage parameters including storage capacity and kinetic parameters for $\mathrm{NH}_{3}$ adsorption and desorption reactions on each storage site of the model. The model simulated $\mathrm{NH}_{3}$ concentrations at the SCR outlet compared to the experimental measurements for the space velocity of $60,000 \mathrm{hr}^{-1}$ are given in Figure 5.5. The model predicted SCR outlet $\mathrm{NH}_{3}$ concentration agrees with the experimental measurements with a cost function value of 5.7. The comparison results confirm that the model is able to correctly predict the $\mathrm{NH}_{3}$ adsorption and desorption under both isothermal and transient temperature conditions.

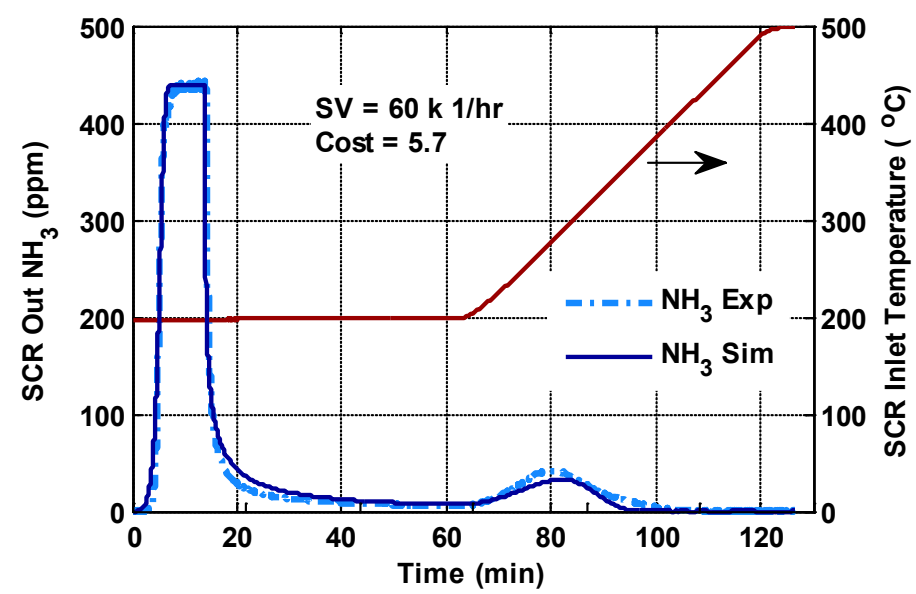

Figure 5.5: Comparison of SCR outlet $\mathrm{NH}_{3}$ between model simulation and TPD reactor experimental results for the space velocity of $60,000 \mathrm{hr}^{-1}$

Besides predicting the SCR outlet gaseous concentrations, the model is able to predict the SCR internal states including the $\mathrm{NH}_{3}$ coverage fraction and $\mathrm{NH}_{3}$ storage on the catalyst which cannot be directly measured. The $\mathrm{NO}_{x}$ reduction performance of the SCR highly depends on the amount $\mathrm{NH}_{3}$ stored on the catalyst. Correctly predicting the $\mathrm{NH}_{3}$ storage is important for model based control and SCR state estimation strategy development. The model predicted $\mathrm{NH}_{3}$ coverage fractions on each site of the model for the space velocity of $60,000 \mathrm{hr}^{-1}$ are given in Figure 5.6. Before $\mathrm{NH}_{3}$ saturation at 8 minutes, the coverage fraction on both sites keeps increasing as $\mathrm{NH}_{3}$ is loaded on the 
catalyst. After the $\mathrm{NH}_{3}$ was turned off at 15 minutes, the $\mathrm{NH}_{3}$ coverage fractions on both sites started to decrease. The $1^{\text {st }}$ site desorbs faster than the $2^{\text {nd }}$ site because the $1^{\text {st }}$ site has a higher $\mathrm{NH}_{3}$ desorption rate. At 65 minutes, about $90 \%$ of the $\mathrm{NH}_{3}$ stored on the $1^{\text {st }}$ site was desorbed, and only about $50 \%$ of the $\mathrm{NH}_{3}$ stored on the $2^{\text {nd }}$ site was desorbed. As temperature was increased, the $\mathrm{NH}_{3}$ desorption from both sites was accelerated and resulted in a bump in the outlet $\mathrm{NH}_{3}$ concentration shown in Figure 5.5. The $\mathrm{NH}_{3}$ coverage fraction on both sites dropped to zero after the stored $\mathrm{NH}_{3}$ completely desorbed from the catalyst. This ability of predicting the $\mathrm{NH}_{3}$ coverage fraction which cannot be directly measured is valuable for the OBD and control applications.

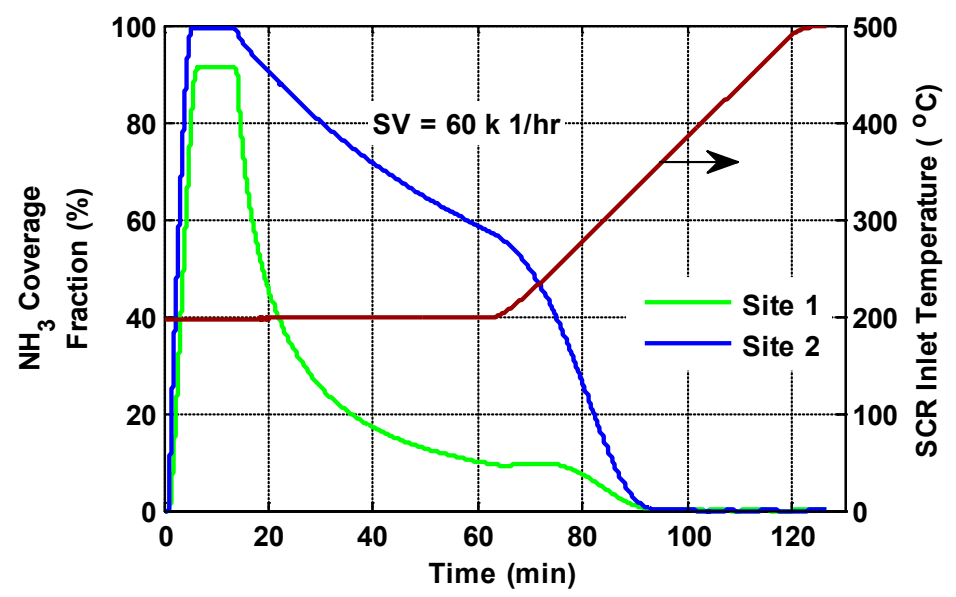

Figure 5.6: Model simulated $\mathrm{NH}_{3}$ coverage fraction for TPD reactor test for the space velocity of $60,000 \mathrm{hr}^{-1}$

The model simulated $\mathrm{NH}_{3}$ concentrations at $\mathrm{SCR}$ outlet compared to the experimental measurements as well as the predicted $\mathrm{NH}_{3}$ coverage fractions on the storage sites for the space velocities of 90,000 and $120,000 \mathrm{hr}^{-1}$ are given in Appendix $\mathrm{H}$. Similar agreement was observed between simulated and experimental results for different space velocities, indicating the space velocity effects on the $\mathrm{NH}_{3}$ adsorption and desorption performance were captured by the model. 


\subsubsection{Model Simulation of Isothermal Reactor Experiments}

The performance of the model calibrated to the reactor experimental data was further validated by running simulations over the full isothermal protocol for each tested temperature and space velocity. The model parameters form the calibration to the reactor data shown in Table 5.1 were used in the model. The activation energies in the middle of the confidence intervals determined for each reaction were used for the SCR reactions except the slow $\mathrm{SCR}$ and the $\mathrm{N}_{2} \mathrm{O}$ formation reactions. Because only temperature runs above $300^{\circ} \mathrm{C}$ were used for calibration of the slow SCR and $\mathrm{N}_{2} \mathrm{O}$ formation reactions, there are more uncertainties in the kinetic parameters in these two reactions. The activation energies for these two reactions were determined by further tuning the parameters in the identified confidence interval to achieve the best simulation results. The model simulated SCR outlet concentrations were compared to the experimental measurements. One typical comparison of $\mathrm{SCR}$ outlet $\mathrm{NO}, \mathrm{NO}_{2}, \mathrm{NH}_{3}$, and $\mathrm{N}_{2} \mathrm{O}$ concentrations for the temperature of $300^{\circ} \mathrm{C}$ and the SV of $90,000 \mathrm{hr}^{-1}$ with the inlet concentrations is shown in Figure 5.7. The simulation results compared to the experimental measurements for other test conditions are shown in Appendix I.

As shown in the figure, the model simulation results agrees with the experimental measurements except a minor over prediction of $\mathrm{NH}_{3}$ slip under the protocol sections with $\mathrm{NO}_{2} / \mathrm{NO}_{x}$ ratio of 0.5 (steps 7 and 9 in Table 3.15). The NO reduction efficiency of the protocol sections with $\mathrm{NO}_{2} / \mathrm{NO}_{x}$ ratio of zero (steps 11 and s3 in Table 3.15) was slightly overestimated. The $\mathrm{N}_{2} \mathrm{O}$ formation and $\mathrm{NO}_{2}$ reduction efficiency of the protocol sections with $\mathrm{NO}_{2} / \mathrm{NO}_{x}$ ratio of 1.0 (steps 16 and 18 in Table 3.15) was also slightly overestimated. The overall model prediction agrees well with the experimental data during both SCR steady state and transient sections when SCR inlet compositions were changed with a maximum simulation error of $25 \mathrm{ppm}$ observed for NO. The maximum simulation errors for $\mathrm{NO}_{2}, \mathrm{NH}_{3}$ and $\mathrm{N}_{2} \mathrm{O}$ are 20,10 and 5 ppm respectively. 


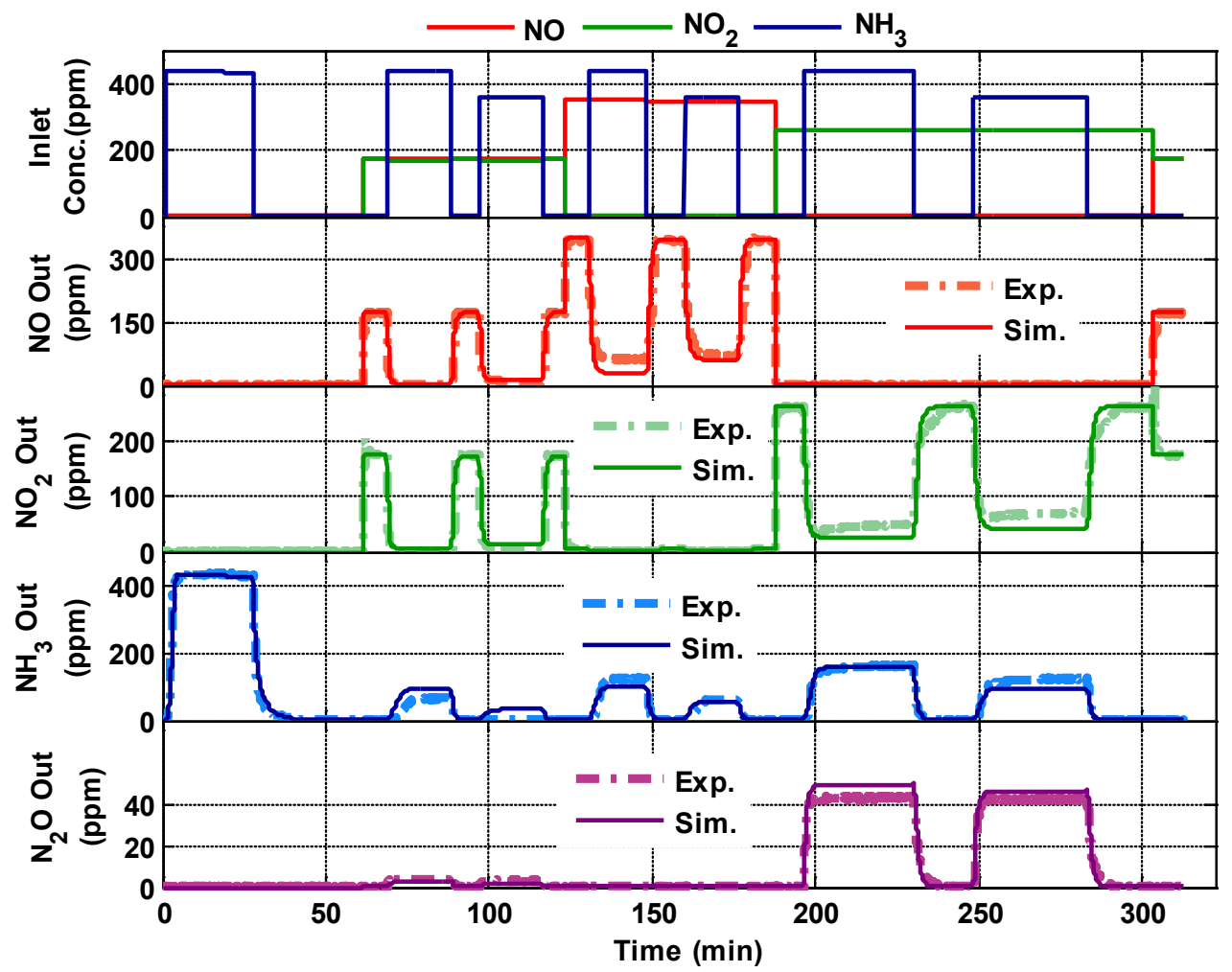

Figure 5.7: SCR outlet concentrations of $\mathrm{NO}, \mathrm{NO}_{2}, \mathrm{NH}_{3}$, and $\mathrm{N}_{2} \mathrm{O}$, comparison between SCR model simulation and experimental measurements. Test conditions: $\mathrm{SV}=90,000$ $\mathrm{hr}^{-1}$, Temperature $=300^{\circ} \mathrm{C}$

The cost function values were used to quantify the agreements between the model simulation results and the experimental measurements. The cost function values of each gas species for all test conditions are given in Table 5.2. It can be observed that all simulation runs give cost function values comparable to the run shown in Figure 5.7 $\left(90,000 \mathrm{hr}^{-1}, 300^{\circ} \mathrm{C}\right)$ except the low temperature test conditions (highlighted) as discussed in Appendix G. This means that the model gives similar agreement with the experimental measurements of the SCR outlet gaseous concentrations under different space velocity and temperature conditions. The space velocity and temperature effects on the reaction rates and the SCR performance are captured by the model. 
Table 5.2: Cost function values for each gas species from comparison of SCR outlet concentrations between model simulation and experimental measurements

\begin{tabular}{|c|c|c|c|c|c|c|}
\hline \multirow{2}{*}{$\begin{array}{c}\text { SV } \\
\left(h r^{-1}\right)\end{array}$} & \multirow{2}{*}{$\begin{array}{c}\text { Temp. } \\
\left({ }^{\circ} \mathrm{C}\right)\end{array}$} & \multicolumn{5}{|c|}{ Cost function value for species } \\
\hline & & $\mathrm{NH}_{3}$ & NO & $\mathrm{NO}_{2}$ & $\mathrm{~N}_{2} \mathrm{O}$ & Total \\
\hline \multirow{5}{*}{60,000} & 200 & 30.1 & 31.5 & 20.0 & 24.0 & 106 \\
\hline & 250 & 30.9 & 5.70 & 26.6 & 36.1 & 99.3 \\
\hline & 300 & 8.79 & 4.79 & 5.08 & 6.05 & 24.7 \\
\hline & 350 & 11.6 & 4.12 & 3.95 & 4.94 & 24.7 \\
\hline & 400 & 12.0 & 6.84 & 4.72 & 6.15 & 29.7 \\
\hline \multirow{10}{*}{90,000} & 200 & 53.8 & 34.4 & 29.5 & 32.6 & 150 \\
\hline & 225 & 17.6 & 13.0 & 19.2 & 25.7 & 75.6 \\
\hline & 250 & 23.7 & 4.93 & 20.6 & 27.5 & 76.7 \\
\hline & 275 & 30.9 & 6.44 & 23.8 & 30.1 & 91.3 \\
\hline & 300 & 10.4 & 5.59 & 8.41 & 9.85 & 34.2 \\
\hline & 325 & 10.2 & 7.57 & 3.81 & 4.45 & 26.0 \\
\hline & 350 & 10.4 & 6.86 & 4.30 & 5.20 & 26.8 \\
\hline & 375 & 10.8 & 6.05 & 4.25 & 5.38 & 26.5 \\
\hline & 400 & 12.3 & 5.81 & 4.87 & 6.21 & 29.2 \\
\hline & 425 & 12.1 & 6.62 & 4.73 & 6.09 & 29.5 \\
\hline \multirow{5}{*}{120,000} & 200 & 51.4 & 47.6 & 34.3 & 37.1 & 170 \\
\hline & 250 & 55.2 & 22.5 & 20.4 & 22.1 & 120 \\
\hline & 300 & 13.4 & 7.08 & 9.79 & 11.4 & 41.7 \\
\hline & 350 & 11.1 & 6.72 & 3.90 & 4.70 & 26.4 \\
\hline & 400 & 14.9 & 6.62 & 5.47 & 6.89 & 33.9 \\
\hline 60,000 & TPD & 5.70 & 0.00 & 0.00 & 0.00 & 5.70 \\
\hline 90,000 & TPD & 4.54 & 0.00 & 0.00 & 0.00 & 4.54 \\
\hline 120,000 & TPD & 3.99 & 0.00 & 0.00 & 0.00 & 3.99 \\
\hline
\end{tabular}

The $\mathrm{NH}_{3}$ storage predicted by the model was also validated through comparisons with the experimental results. The $\mathrm{NH}_{3}$ storage on the catalyst under the equilibrium state of certain steps of the test protocol was calculated from experimental results and are given in Figure 5.8. The model simulation results are also shown in the figure for comparison. The $\mathrm{x}$ axes of the figure refers to the step number of the isothermal reactor test protocol. Step 2 is the $\mathrm{NH}_{3}$ inventory section before $\mathrm{O}_{2}$ was turned on. $\mathrm{NH}_{3}$ oxidation reactions take place at step 3 and less $\mathrm{NH}_{3}$ is stored compared to step 2. Steps 6 and 8 are the protocol sections with the $\mathrm{NO}_{2} / \mathrm{NO}_{x}$ ratio of 0.5 and two different ANRs. Steps 11 and 13 are the protocol sections with $\mathrm{NO}_{2} / \mathrm{NO}_{\mathrm{x}}$ ratio of 0.0 and two different ANRs. Steps 16 and 
18 are the protocol sections with $\mathrm{NO}_{2} / \mathrm{NO}_{x}$ ratio of 1.0. It can be seen that the $\mathrm{NH}_{3}$ stored during steps 6 to 18 are significantly less than step 2 because part of the $\mathrm{NH}_{3}$ stored is being consumed by the SCR reactions. The model predicted $\mathrm{NH}_{3}$ storage is comparable with the experimental results with the maximum simulation error of $11 \mathrm{gmol} / \mathrm{m}^{3}$.

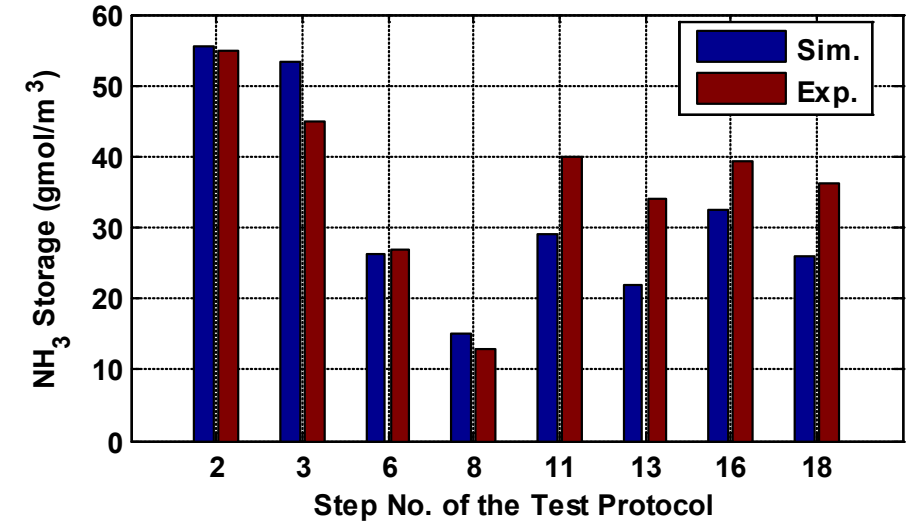

Figure 5.8: Calculated $\mathrm{NH}_{3}$ stored on the catalyst during certain steps of the reactor protocol for the SV of $90,000 \mathrm{hr}^{-1}$ and the temperature of $300^{\circ} \mathrm{C}$

\subsection{SCR Model Calibrated to Engine Data}

The model parameters identified from the steady state engine experimental data were shown in Table 5.1 in Section 5.2. The model simulations results were validated against both the steady state and the transient engine experimental data

\subsubsection{Model Simulation of Steady State Engine Experiments}

The performance of the SCR model with calibration to the steady state engine data was validated by running the simulation with a full set of identified parameters for each test condition and comparing the simulated SCR outlet concentrations of $\mathrm{NO}, \mathrm{NO}_{2}$, and $\mathrm{NH}_{3}$ with experimental measurements. The cost function values for different gaseous species were used to evaluate the agreement between the simulation results and experimental measurements. 
One comparison between the model simulation and experimental measurements of SCR outlet gaseous concentrations under the temperature of $376^{\circ} \mathrm{C}$ is given in Figure 5.9. The top subplot of the figure shows the SCR inlet concentrations of $\mathrm{NO}, \mathrm{NO}_{2}$ and $\mathrm{NH}_{3}$. The bottom three subplots show the SCR outlet concentrations of $\mathrm{NO}, \mathrm{NO}_{2}$ and $\mathrm{NH}_{3}$ compared between the model simulation and the experimental results. The $\mathrm{NH}_{3}$ prediction from the SCR model based on the engine data shown as Sim.1 was post processed by the developed sample line model to take into consideration the $\mathrm{NH}_{3}$ adsorption/desorption in the sample line. The simulated $\mathrm{NH}_{3}$ processed by the sample line model is shown as Sim.2. It can be seen from Figure 5.9 that the simulation results follow the overall trend of the experimental measurements for $\mathrm{NO}$ and $\mathrm{NO}_{2}$. The model is able to simulate the SCR outlet $\mathrm{NO}$ and $\mathrm{NO}_{2}$ concentrations under both steady state and transient DEF injection conditions when it is switched between different DEF injection rates. The maximum simulation error for $\mathrm{NO}$ and $\mathrm{NO}_{2}$ is $19 \mathrm{ppm}$. The model simulated $\mathrm{NH}_{3}$ (Sim1) did not agree with the measured $\mathrm{NH}_{3}$ concentration from the IMR-MS, and it was determined to be caused by the $\mathrm{NH}_{3}$ adsorption and desorption phenomenon in the sample line. The simulated $\mathrm{NH}_{3}$ corrected using the sample line model (Sim2) agrees with the time history experimental measurements with the maximum simulation error of $16 \mathrm{ppm}$. 


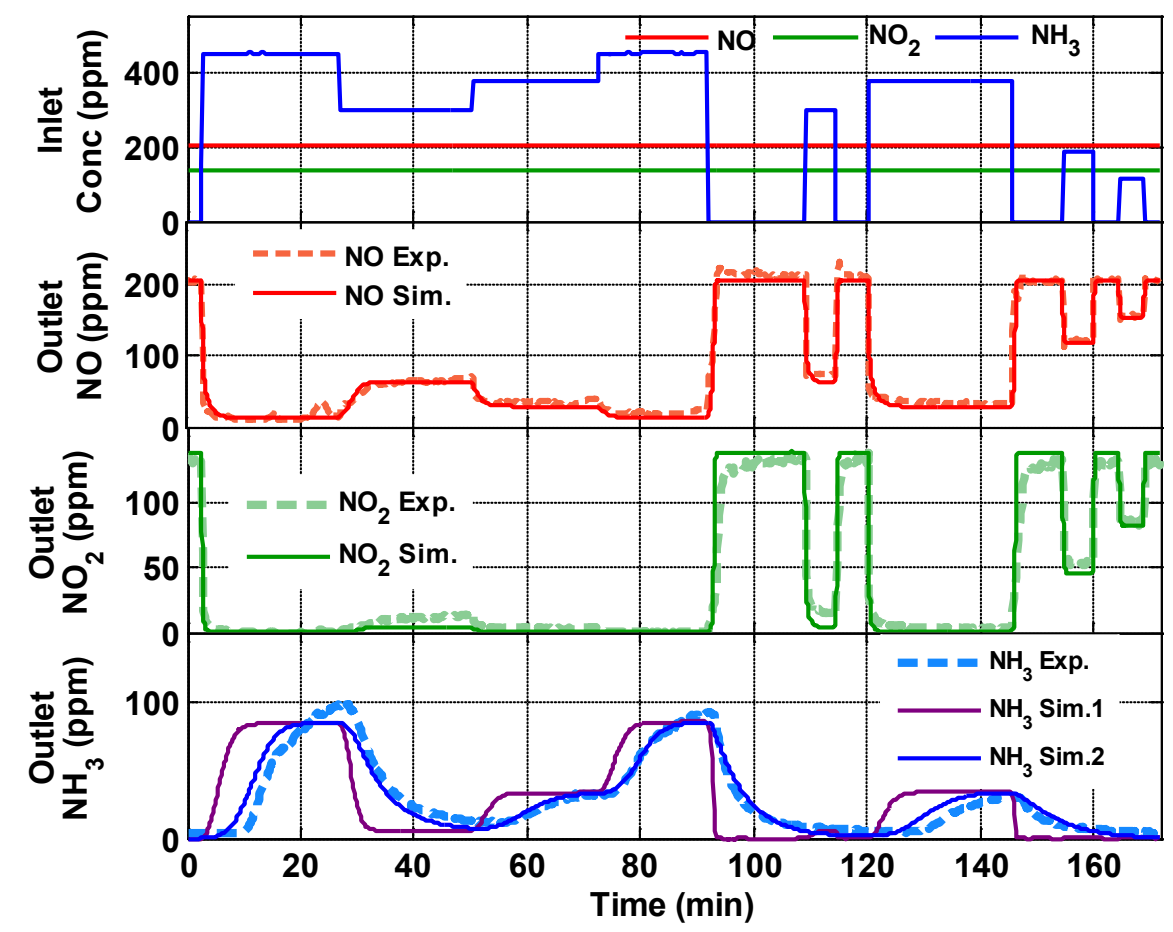

Figure 5.9: Comparison of the SCR outlet gaseous concentrations between simulation results and experimental measurements for test point 2 in Table 3.11 with the temperature of $376^{\circ} \mathrm{C}$. (Sim1: SCR model predicted $\mathrm{NH}_{3}$; $\operatorname{Sim} 2: \mathrm{NH}_{3}$ slip processed by the sample line model)

The cost function values of all simulations for steady state SCR experiments are given in Table 5.3. The cost function value for $\mathrm{NH}_{3}$ from both the SCR model simulation and the sample line model simulation are presented in the table. Since a smaller weighing factor was used for $\mathrm{NH}_{3}$ in the cost function value calculation during the optimization process, the $\mathrm{NH}_{3}$ simulation from the SCR model has a higher level of cost function compared to $\mathrm{NO}$ and $\mathrm{NO}_{2}$. This error was taken care of by the sample line model. It can be observed that the corrected $\mathrm{NH}_{3}$ slip using the sample line model has smaller cost values compared to the SCR model predictions. The total cost values consider the $\mathrm{NH}_{3}$ cost function value from the sample line instead of the SCR model. All runs have a comparable low level of cost function values compared to test point 2 which was shown in Figure 5.9 except test point 1 and its repeat which has high $\mathrm{NO}_{\mathrm{x}}$ and $\mathrm{NH}_{3}$ slip concentrations. The simulation errors are higher for this point. The comparisons between model simulation and experimental results for other test points are given in Appendix J. 
Table 5.3: Cost function values for each gas species of the model simulation with identified model parameter

\begin{tabular}{|c|c|c|c|c|c|}
\hline $\begin{array}{l}\text { Run } \\
\text { No. }\end{array}$ & NO & $\mathrm{NO}_{2}$ & $\begin{array}{c}\mathrm{NH}_{3} \\
\text { SCR } \\
\text { Model }\end{array}$ & $\begin{array}{c}\mathrm{NH}_{3} \\
\text { Sample } \\
\text { Line } \\
\text { Model } \\
\end{array}$ & Total \\
\hline 1 & 18.8 & 10.9 & 18.8 & 13.3 & 43.0 \\
\hline 2 & 6.91 & 5.90 & 17.0 & 5.20 & 18.0 \\
\hline 3 & 3.54 & 4.71 & 11.3 & 7.57 & 15.8 \\
\hline 4 & 4.75 & 5.47 & 7.94 & 5.78 & 16.0 \\
\hline 5 & 4.57 & 10.5 & 10.0 & 6.04 & 21.1 \\
\hline 6 & 3.72 & 6.84 & 5.89 & 4.80 & 15.4 \\
\hline 7 & 6.32 & 7.15 & 3.37 & 3.26 & 16.7 \\
\hline $1^{\prime}$ & 10.1 & 8.60 & 18.2 & 9.95 & 28.6 \\
\hline $4^{\prime}$ & 4.34 & 5.36 & 6.03 & 3.71 & 13.4 \\
\hline
\end{tabular}

\subsubsection{Model Simulation of Transient Engine Experiments}

SCR transient experimental data from the surrogate HD-FTP tests were used to validate the SCR model performance under transient engine conditions. The model parameters were varied in the confidence range determined from the engine data to get the best fit for the transient data. A comparison between model simulation and experimental measurements for transient test 1 shown in Table 3.12 is given in Figure 5.10. The changes in the SCR inlet $\mathrm{NO}$ and $\mathrm{NO}_{2}$ concentrations, temperatures and space velocities for the cycle are shown in Figure 3.16 in Section 3.5. In this test, the SCR inlet $\mathrm{NO}, \mathrm{NO}_{2}$ and $\mathrm{NH}_{3}$ concentrations are shown in the top subplot. The Inlet $\mathrm{NH}_{3}$ concentration was calculated based on the exhaust mass flow rate and the DEF injection rate as described in Appendix $B$. In this test, the DEF injection was manually turned on at 2 minutes to study the $\mathrm{NH}_{3}$ adsorption under transient conditions. The DEF injection was turned off at 15 minutes to allow $\mathrm{NH}_{3}$ desorption under transient conditions. The DEF injection rate between the 2 and 15 minutes was determined by a default control strategy implanted in the engine ECM. The model simulated SCR downstream $\mathrm{NO}, \mathrm{NO}_{2}$, and $\mathrm{NH}_{3}$ concentrations were compared to the experimental measurements as shown in Figure 5.10. The $\mathrm{NH}_{3}$ concentration measurements are from the $\mathrm{NH}_{3}$ sensor and the simulation was run without the sample line model incorporated. It can be seen that the 
simulation results follow the experimental measurements. The simulation errors in NO and $\mathrm{NO}_{2}$ are obvious after 15 minutes when the DEF injection was turned off. The simulated $\mathrm{NH}_{3}$ concentration is also lower than the measured $\mathrm{NH}_{3}$ concentration around 15 minutes. The model predicted species concentrations follow a similar time trend as the experimental measurement results.

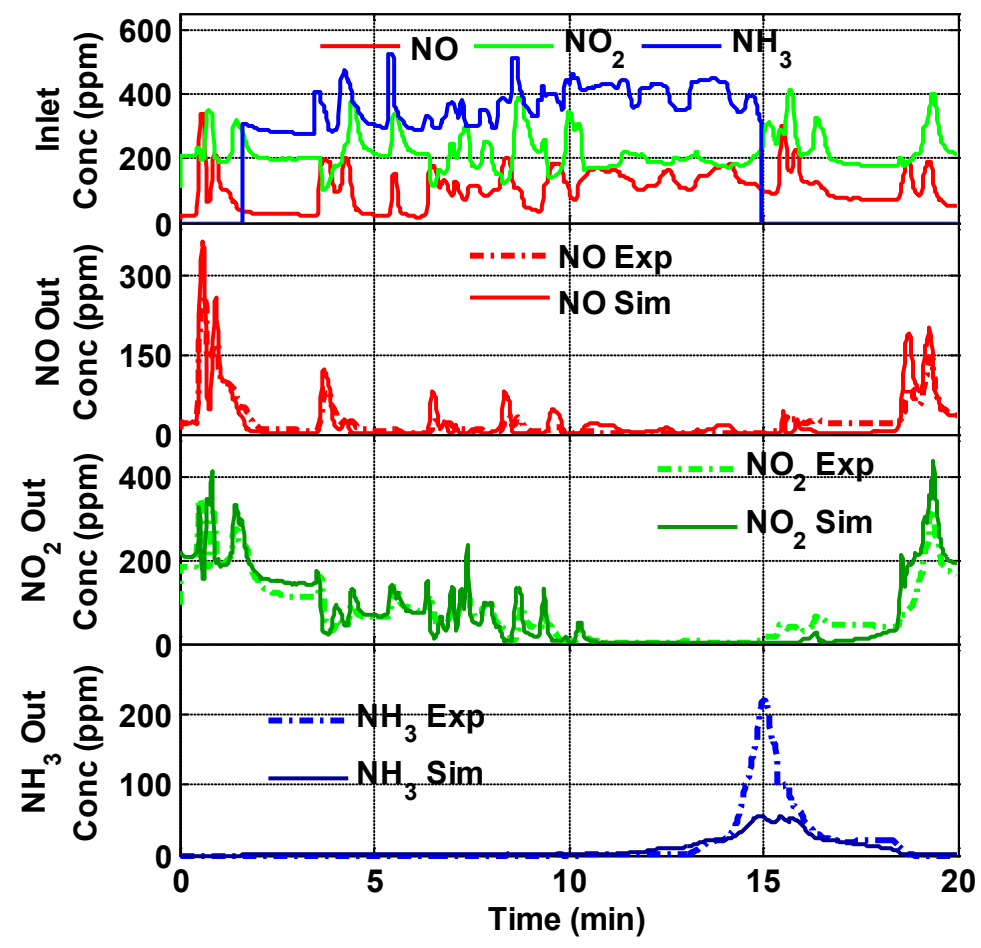

Figure 5.10: Model simulation compared to experimental results for the surrogate HDFTP transient test 1 with manual control of DEF injection

The SCR inlet and outlet cumulative $\mathrm{NO}_{\mathrm{x}}$ and $\mathrm{NH}_{3}$ as well as the $\mathrm{NO}_{\mathrm{x}}$ conversion efficiency of the cycle from both simulation and experiments are shown in Figure 5.11. It can be seen that the model is able to simulate the cumulative SCR outlet $\mathrm{NO}_{x}$ and $\mathrm{NH}_{3}$ with simulation errors of 1.9 and $1.1 \mathrm{~g}$ for $\mathrm{NO}_{\mathrm{x}}$ and $\mathrm{NH}_{3}$ respectively at the end of the cycle. The prediction of $\mathrm{NO}_{x}$ conversion efficiency of the cycle agrees with the experimental results for most of the cycle. The simulation errors mainly exist at the last section of the cycle after the DEF injection was turned off at 15 minutes. The model 
simulation results compared to the experimental measurements for the five other transient SCR experiments are given in Appendix K.

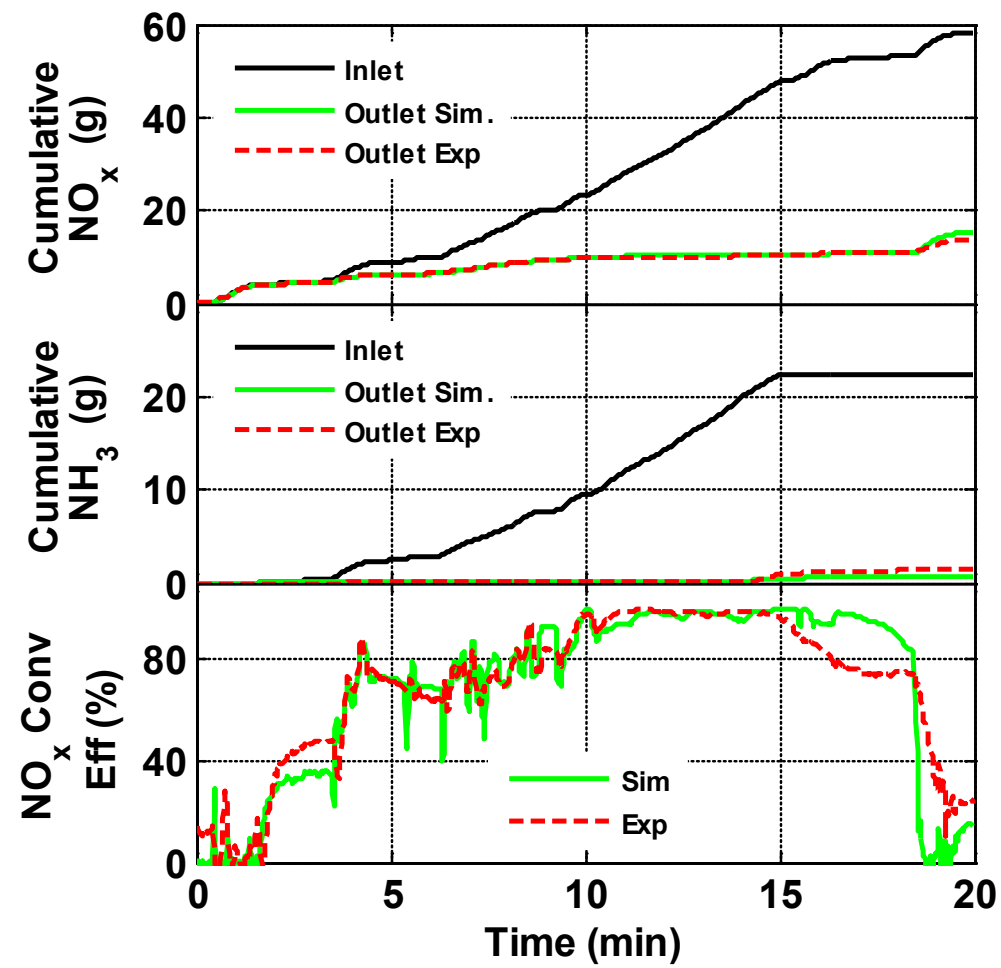

Figure 5.11: Cumulative $\mathrm{NO}_{x}, \mathrm{NH}_{3}$ and $\mathrm{NO}_{x}$ conversion efficiency of the surrogate $\mathrm{HD}-$ FTP cycle, compare between simulation and experimental results for transient test 1

The cost function value of each gas for the transient test 1 shown in Figure 5.10 is given in Table 5.4. The cost function values of each species for each transient test shown in the test matrix of Table 3.12 in Section 3.5 are also given in Table 5.4. Compared to the transient test 1 with results shown in Figure 5.10, higher total cost function values (62.8 compared to 48.4) were observed for transient test 2 with the DEF injection level of 1.2. This is because higher amplitude of $\mathrm{NH}_{3}$ slip was generated because of overdosing of the DEF during this experiment and the difference in model simulated and experimental measured $\mathrm{NH}_{3}$ was also higher. The cost function values from all the experiments are comparable, meaning that similar simulation results were obtained. 
Table 5.4: Cost function values of model simulation compared to experimental measurements for transient tests

\begin{tabular}{|c|c|c|c|c|c|}
\cline { 3 - 6 } \multicolumn{2}{c|}{} & \multicolumn{4}{c|}{ Cost function value } \\
\hline Test No. & $\begin{array}{c}\text { DEF Inj } \\
\text { Level }\end{array}$ & $\mathbf{N H}_{\mathbf{3}}$ & $\mathbf{N O}$ & NO $_{\mathbf{2}}$ & Total \\
\hline 1 & Manual & 11.1 & 13.1 & 24.1 & 48.4 \\
\hline 2 & 1.2 & 46.4 & 8.20 & 8.20 & 62.8 \\
\hline 3 & 1.0 & 10.6 & 9.90 & 9.48 & 30.0 \\
\hline 4 & 0.8 & 5.10 & 11.6 & 13.7 & 30.4 \\
\hline 5 & 0.5 & 0 & 17.8 & 23.7 & 41.5 \\
\hline 6 & 0.3 & 0 & 19.0 & 21.8 & 40.8 \\
\hline
\end{tabular}

Higher errors in $\mathrm{NO}$ and $\mathrm{NO}_{2}$ can be noticed for transient tests 5 and 6 with the DEF injection level of 0.5 and 0.3 respectively. One possible explanation is that the $\mathrm{NO}_{\mathrm{x}}$ reduction performance of the SCR highly depends on the $\mathrm{NH}_{3}$ stored on the catalyst. Under the low level of DEF injection, the $\mathrm{NH}_{3}$ stored on the catalyst is less (shown in Figure 5.12) and is more affected by the changes in SCR space velocity and temperature. Under higher DEF injection level conditions, the $\mathrm{NH}_{3}$ stored on the catalyst is higher, and a slight change in the $\mathrm{NH}_{3}$ storage resulting from a change in the space velocity and temperature has relatively less of an effect on the $\mathrm{NO}_{\mathrm{x}}$ conversion efficiency. The cost function values in $\mathrm{NH}_{3}$ of transient tests 5 and 6 are 0 because no $\mathrm{NH}_{3}$ slip was formed for both model simulation and experiments. Other factors including repeatability of the cycle, analyzer measurement error, and neglecting several SCR reactions $\left(\mathrm{N}_{2} \mathrm{O}\right.$ formation, $2^{\text {nd }} \mathrm{NH}_{3}$ oxidation reaction and $\mathrm{NO}$ oxidation reaction) in the model also contribute to the difference between model simulation and experimental results. 


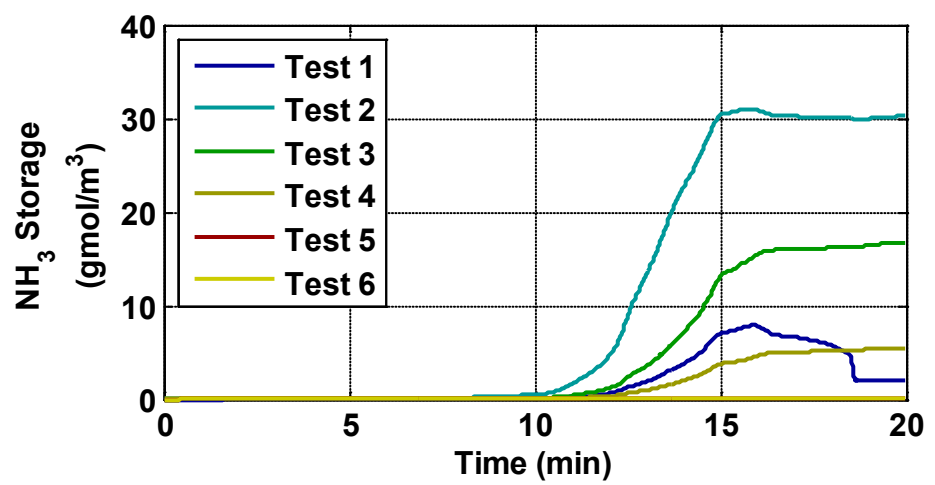

Figure 5.12: Model predicted $\mathrm{NH}_{3}$ storage on the catalyst during surrogate HD-FTP cycle tests 1-6

The model predicted SCR outlet temperatures compared to the experimental measurements as well as the measured inlet temperatures for the surrogate HD-FTP test are given in Figure 5.13. The subplot (a) shows the measured SCR inlet and outlet temperatures compared with the model predicted SCR outlet temperatures. It can be seen that the outlet temperature follows a similar trend as the inlet temperature. $A$ maximum temperature difference of $30^{\circ} \mathrm{C}$ was observed. The differences were caused by the heat transfer between the gas flow, the substrate and the ambient. The heat transfer equations with the heat transfer coefficients identified from the temperature data were incorporated in the SCR model to simulate the outlet temperature (Section 4.1.3). The coefficients for heat transfer between gas flow and the substrate and between the substrate and the ambient were determined to be 13.5 and $0.015 \mathrm{~W} / \mathrm{m}^{2} / \mathrm{K}$ respectively. The subplot (b) shows the error between the simulated and experimentally measured SCR outlet temperatures $\left(T_{S i m}-T_{\text {Exp }}\right)$. It can be seen that the model predictions follow the same trend as the measured outlet temperature. The amplitude and the response to the change in the inlet temperature were correctly predicted with simulation errors within the $\pm 5^{\circ} \mathrm{C}$ range for most of the cycle. The biggest error is about $8^{\circ} \mathrm{C}$ around 18 minutes in the cycle.

The results showed that the model is able to predict the SCR outlet temperatures under transient conditions. By considering the heat transfer in the SCR model, the axial 
temperature gradients in the SCR catalyst can be considered in the different axial model elements (described in Section 4.1.3), thus improving the simulation accuracy.
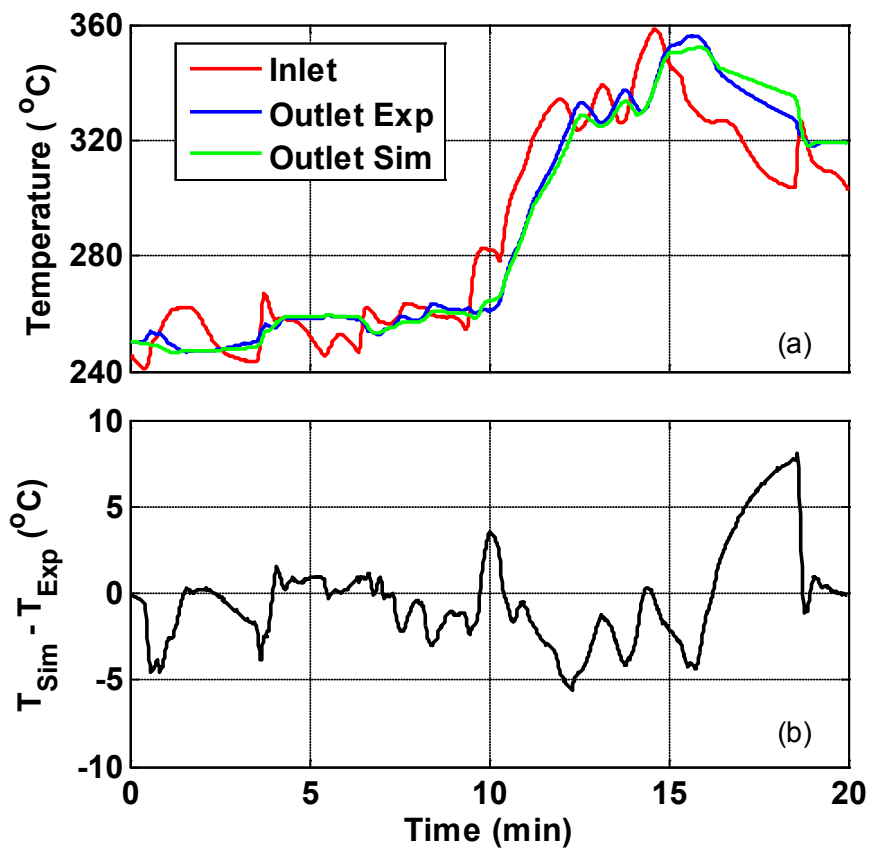

Figure 5.13: Model predicted SCR outlet temperature for the surrogate HD-FTP test

\subsubsection{Simulation of $\mathrm{NO}_{2} / \mathrm{NO}_{x}$ Ratio Effects}

The $\mathrm{NO}_{2} / \mathrm{NO}_{x}$ ratio at the SCR inlet is an important factor that affects the $\mathrm{NO}_{\mathrm{x}}$ reduction performance of the SCR. In engine applications with DOC and CPF installed before the $\mathrm{SCR}$, the $\mathrm{NO}_{2} / \mathrm{NO}_{x}$ ratio going into the SCR is dependent on the DOC oxidation performance as well as the passive regeneration process in the CPF[131]. Reference [132] indicated that aging of the $\mathrm{DOC}$ lowers the $\mathrm{NO}$ to $\mathrm{NO}_{2}$ conversion efficiency across the DOC and changes the $\mathrm{NO}_{2} / \mathrm{NO}_{\mathrm{x}}$ ratio at the SCR inlet. In this study, the $\mathrm{NO}_{2} / \mathrm{NO}_{\mathrm{x}}$ ratio effects on the $\mathrm{NO}_{x}$ reduction performance, $\mathrm{NH}_{3}$ slip and $\mathrm{NH}_{3}$ storage under transient conditions were investigated through model simulations with the SCR model calibrated to the engine data. 
The model was setup to simulate the SCR performance during the surrogate HD-FTP cycle. The model inlet $\mathrm{NO}_{\mathrm{x}}$ concentrations were set to be equal to the inlet $\mathrm{NO}_{\mathrm{x}}$ measurements during the cycle test while the $\mathrm{NO}_{2} / \mathrm{NO}_{\mathrm{x}}$ ratio was varied from 0 to 1 in the model. The temperature and exhaust mass flow rate measured from the transient test remained in the model. A constant inlet ANR of 1 was used for different $\mathrm{NO}_{2} / \mathrm{NO}_{\mathrm{x}}$ ratios. The cumulative $\mathrm{NO}_{x}$ and $\mathrm{NH}_{3}$ at both SCR inlet and outlet, $\mathrm{NH}_{3}$ stored on the catalyst and the overall $\mathrm{NO}_{\mathrm{x}}$ reduction efficiency were quantified for each $\mathrm{NO}_{2} / \mathrm{NO}_{\mathrm{x}}$ ratio simulation.

The simulation results are summarized and given in Table 5.5. The cumulative inlet $\mathrm{NH}_{3}$ is constant for all runs. The cumulative mass of the inlet $\mathrm{NO}_{\mathrm{x}}$ increases with increased $\mathrm{NO}_{2} / \mathrm{NO}_{\mathrm{x}}$ ratio because of the higher molecular weight for $\mathrm{NO}_{2}$ compared to $\mathrm{NO}$ and the increasing fraction of $\mathrm{NO}_{2}$. The cumulative SCR outlet $\mathrm{NH}_{3}$ is 1.70 grams for the $\mathrm{NO}_{2} / \mathrm{NO}_{\mathrm{x}}$ ratio of 0 and it decreases with the $\mathrm{NO}_{2} / \mathrm{NO}_{\mathrm{x}}$ ratio approaching 0.5 . The outlet $\mathrm{NH}_{3}$ is lowest for the $\mathrm{NO}_{2} / \mathrm{NO}_{x}$ ratio greater than 0.6. The $\mathrm{NH}_{3}$ stored on the catalyst at the end of the cycle dropped from 3.4 to 0.08 grams when the inlet $\mathrm{NO}_{2} / \mathrm{NO}_{\mathrm{x}}$ ratio was varied from 0 to 1 . The fast SCR reaction is becoming dominant when the $\mathrm{NO}_{2} / \mathrm{NO}_{\mathrm{x}}$ ratio approaches 0.5 and the greater reaction rate of the fast SCR reaction compared to the standard SCR reaction results in a higher consumption rate of the stored $\mathrm{NH}_{3}$. When the $\mathrm{NO}_{2} / \mathrm{NO}_{\mathrm{x}}$ ratio approaches 1 , the slow SCR reaction becomes dominant. The stored $\mathrm{NH}_{3}$ still decreases although the reaction rate for the slow SCR reaction is low. This is because the slow SCR reaction with a stoichiometric $\mathrm{NH}_{3} / \mathrm{NO}_{\mathrm{x}}$ ratio is higher than 1 (1.33) and consumes more $\mathrm{NH}_{3}$ to reduce the amount of $\mathrm{NO}_{2}$. The cumulative outlet $\mathrm{NO}_{\mathrm{x}}$ and the overall $\mathrm{NO}_{x}$ conversion efficiency of the cycle is highest with the $\mathrm{NO}_{2} / \mathrm{NO}_{\mathrm{x}}$ ratio of 0.5 , meaning that the optimal $\mathrm{NO}_{\mathrm{x}}$ reduction performance under the surrogate $\mathrm{HD}$ FTP transient conditions is achieved with a $\mathrm{NO}_{2} / \mathrm{NO}_{x}$ ratio of 0.5 .

This conclusion leads one to envision an engine-aftertreatment system model for the $\mathrm{ECU}$ that would control the SCR inlet $\mathrm{NO}_{2} / \mathrm{NO}_{x}$ ratio to around 0.5 in order to achieve the highest $\mathrm{NOx}$ conversion efficiency with minimum $\mathrm{NH}_{3}$ slip. 
Table 5.5: Simulation results of $\mathrm{NO}_{2} / \mathrm{NO}_{\mathrm{x}}$ ratio effects

\begin{tabular}{|c|c|c|c|c|c|c|}
\hline $\begin{array}{c}\text { Inlet } \\
\mathbf{N O}_{2} / \mathbf{N O}_{\mathbf{x}} \\
\text { Ratio }\end{array}$ & $\begin{array}{c}\text { Inlet } \\
\mathbf{N H}_{\mathbf{3}}\end{array}$ & $\begin{array}{c}\text { Inlet } \\
\mathbf{N} \mathbf{O}_{\mathbf{x}}\end{array}$ & $\begin{array}{c}\text { Outlet } \\
\mathbf{N H}_{\mathbf{3}}\end{array}$ & $\begin{array}{c}\text { Outlet } \\
\mathbf{N O}_{\mathbf{x}}\end{array}$ & $\begin{array}{c}\mathbf{N H}_{\mathbf{3}} \\
\text { Stored }\end{array}$ & $\begin{array}{c}\mathbf{N} \mathbf{O}_{\mathbf{x}} \text { Conversion } \\
\text { Efficiency }\end{array}$ \\
\hline 0.0 & 24.9 & $\mathbf{( g )}$ & $\mathbf{( g )}$ & $\mathbf{( g )}$ & $\mathbf{( g )}$ & $\mathbf{( \% )}$ \\
\hline 0.1 & 24.9 & 46.4 & 1.70 & 15.7 & 3.40 & 64.3 \\
\hline 0.2 & 24.9 & 48.7 & 0.96 & 11.5 & 2.32 & 76.4 \\
\hline 0.3 & 24.9 & 51.1 & 0.63 & 9.30 & 1.69 & 81.8 \\
\hline 0.4 & 24.9 & 53.4 & 0.38 & 7.40 & 1.08 & 86.1 \\
\hline 0.5 & 24.9 & 55.8 & 0.22 & 6.60 & 0.60 & 88.2 \\
\hline 0.6 & 24.9 & 58.1 & 0.18 & 7.80 & 0.33 & 86.6 \\
\hline 0.7 & 24.9 & 60.5 & 0.19 & 10.6 & 0.21 & 82.5 \\
\hline 0.8 & 24.9 & 62.8 & 0.20 & 14.0 & 0.13 & 77.7 \\
\hline 0.9 & 24.9 & 65.1 & 0.20 & 17.6 & 0.09 & 73.0 \\
\hline 1.0 & 24.9 & 67.5 & 0.19 & 21.2 & 0.08 & 68.6 \\
\hline
\end{tabular}

\section{4 $\mathrm{NH}_{3}$ Maldistribution Data and Simulation of Maldistribution Effects}

As discussed in Section 5.1, The $\mathrm{NH}_{3}$ maldistribution at the SCR inlet is considered to be one of the reasons causing the differences in kinetic parameters between the model calibrations developed based on reactor and engine experimental data. In this section, the $\mathrm{NH}_{3}$ maldistribution level at the SCR inlet was quantified. The $\mathrm{NH}_{3}$ maldistribution effects on the model parameters as well as on the SCR performance in terms of $\mathrm{NO}_{\mathrm{x}}$ conversion efficiency and $\mathrm{NH}_{3}$ slip are studied and discussed in this section.

\subsection{1 $\mathrm{NH}_{3}$ Maldistribution Experimental Results}

The experimental setup and test procedure were introduced in Section 3.7 and the $\mathrm{NH}_{3}$ maldistribution test matrix is shown in Table 3.13. The uniformity index (UI) shown in Equation 5.1 was introduced to quantify the nonuniformity of the species concentrations. 


$$
U I=\frac{\sqrt{\sum_{i=1}^{n}\left(x_{i}-\bar{x}\right)^{2} / n}}{\bar{x}}
$$

Where, $x_{i}$ is the gaseous concentration measurement at each location. $\bar{x}$ is the average of all concentration measurements at different locations, and $n$ is the total number of measurements. All measurement points, except the center location, were used in the UI calculation. An UI of 1.0 means the distribution is uniform and lower UI means less uniformity. The data process procedures and test results for test point 1 shown in Table 3.13 are described as follows.

The distributions of $\mathrm{NO}, \mathrm{NO}_{2}$ and $\mathrm{NO}_{x}$ concentrations downstream of the SCR without the DEF injection $(A N R=0)$ for test point 1 are given in Figures 5.14-5.16. It can be observed that the $\mathrm{UI}$ for $\mathrm{NO}, \mathrm{NO}_{2}$ and $\mathrm{NO}_{\mathrm{x}}$ are $0.99,0.98$ and 0.99 respectively. All the measurements are within the \pm 5 ppm confidence range of the analyzer, indicating that the $\mathrm{NO}_{\mathrm{x}}$ distribution without the DEF injection is uniform. The test point 2 also showed the similar results for the distribution of $\mathrm{NO}_{\mathrm{x}}$ without the upstream DEF injection. As a result, it was safe to assume uniform distribution of $\mathrm{NO}_{x}$ without upstream DEF injection. For the other test conditions in Table 3.13, only downstream $\mathrm{NO}_{\mathrm{x}}$ distribution during the DEF injection was measured.

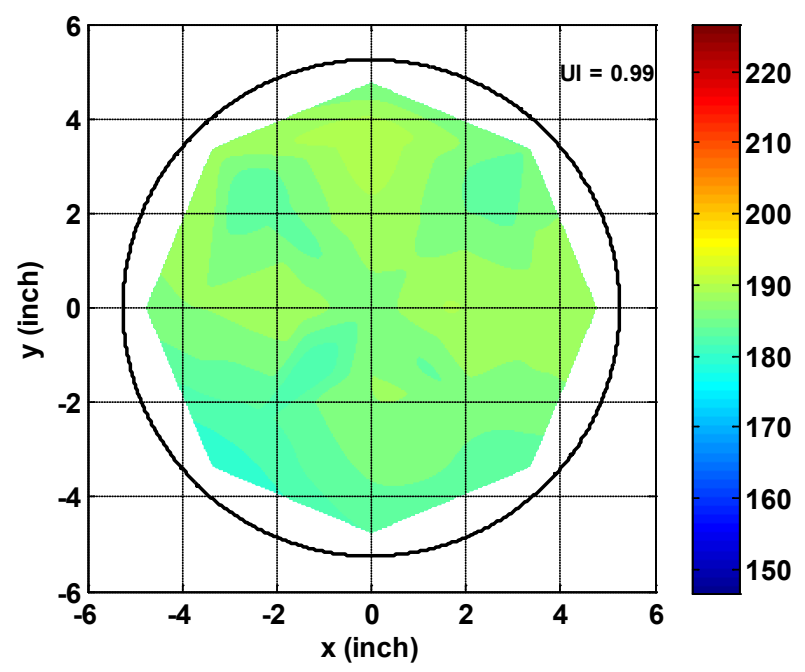

Figure 5.14: Distribution profile of NO with the average ANR of 0 for test point 1 


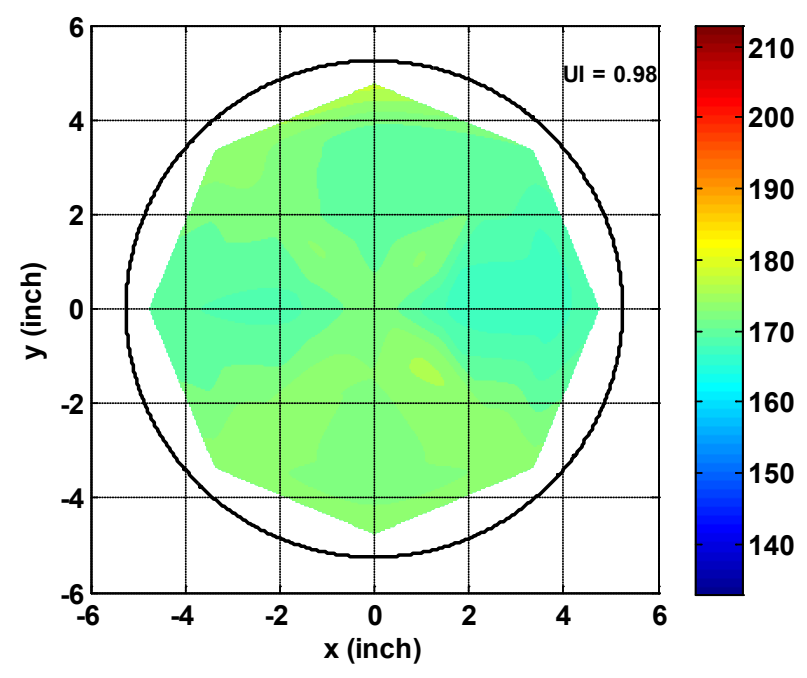

Figure 5.15: Distribution profile of $\mathrm{NO}_{2}$ with the average ANR of 0 for test point 1

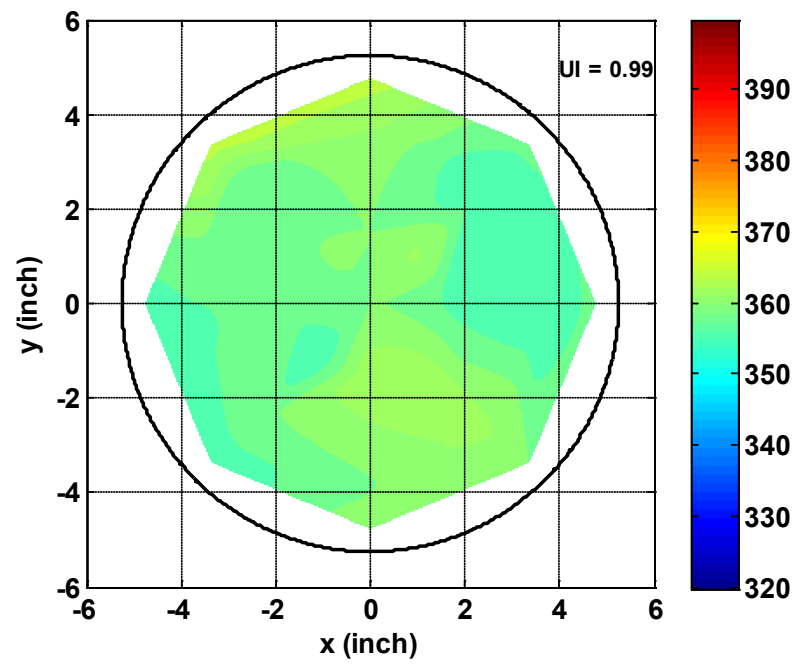

Figure 5.16: Distribution profile of $\mathrm{NO}_{\mathrm{x}}$ with the average ANR of 0 for test point 1

The distributions of $\mathrm{NO}, \mathrm{NO}_{2}$ and $\mathrm{NO}_{x}$ concentrations downstream of the SCR with the DEF injection (ANR $=0.3$ ) for test point 1 shown in Table 3.13 are given in Figures 5.175.19. The nonuniformity for $\mathrm{NO}, \mathrm{NO}_{2}$ and $\mathrm{NO}_{x}$ with the $\mathrm{UI}$ of $0.93,0.89$ and 0.91 were determined. By comparing the distribution profiles determined with and without the DEF 
injection, it can be concluded that the maldistribution of $\mathrm{NH}_{3}$ generated from the injected DEF caused the nonuniformity.

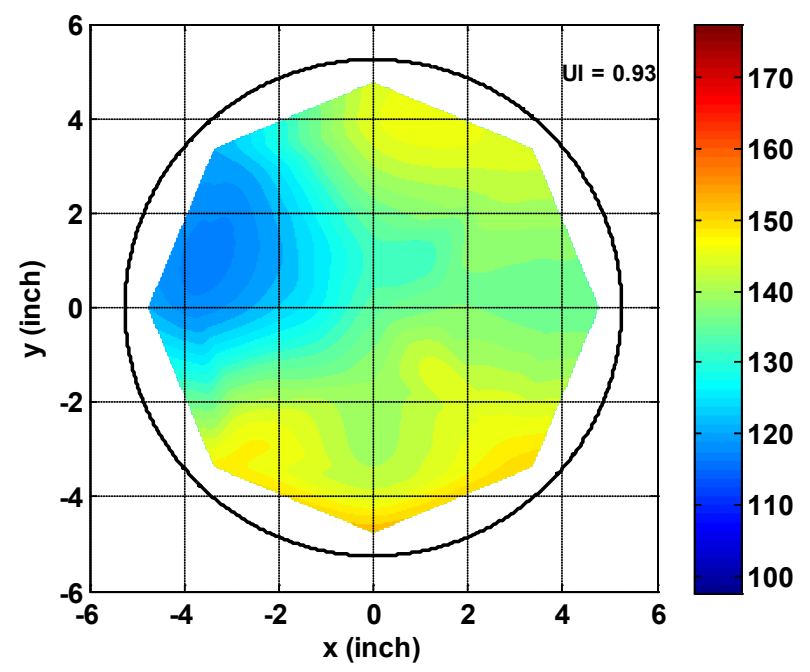

Figure 5.17: Distribution profile of NO with the average ANR of 0.3 for test point 1

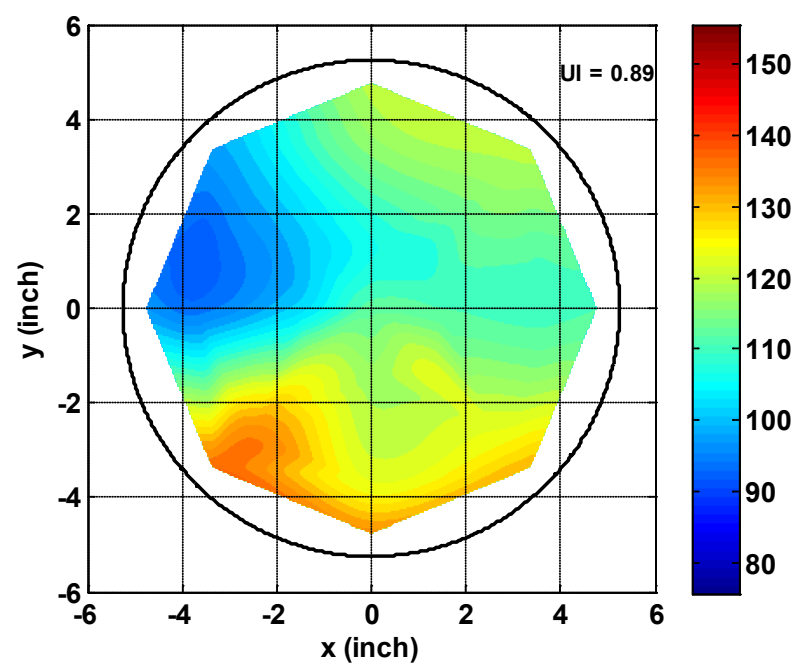

Figure 5.18: Distribution profile of $\mathrm{NO}_{2}$ with the average ANR of 0.3 for test point 1 


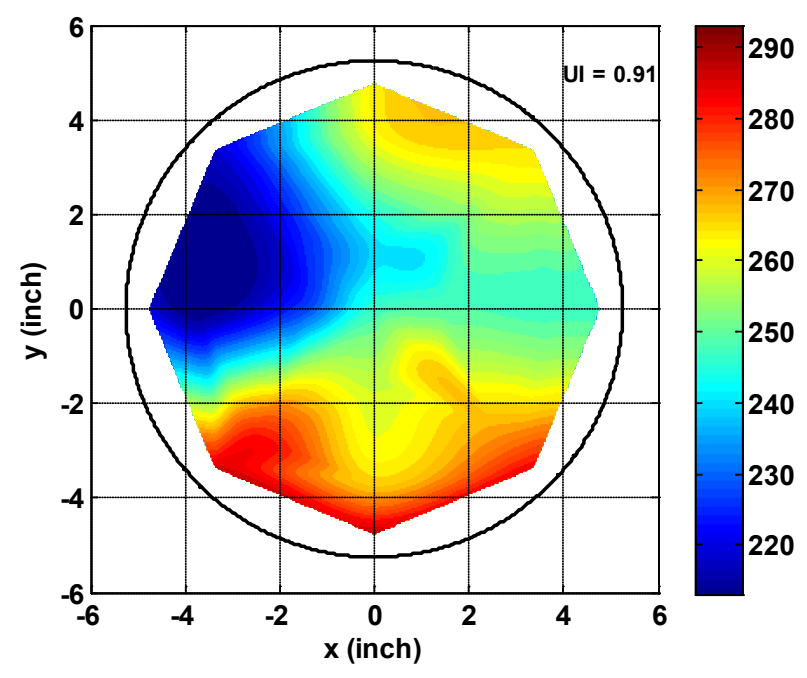

Figure 5.19: Distribution profile of $\mathrm{NO}_{\mathrm{x}}$ with the average ANR of 0.3 for test point 1

The differences between the $\mathrm{NO}_{x}$ distribution profiles with and without the DEF injection shown in Figures 5.16 and 5.19 were considered to be the result of the distribution of $\mathrm{NH}_{3}$ at the SCR inlet. The determined distribution of $\mathrm{NH}_{3}$ for the average ANR of 0.3 with the $\mathrm{UI}$ of 0.79 is shown in Figures 5.20. The determined distribution of ANR is shown in Figure 5.21 with a UI of 0.79 with the average ANR of 0.3 .

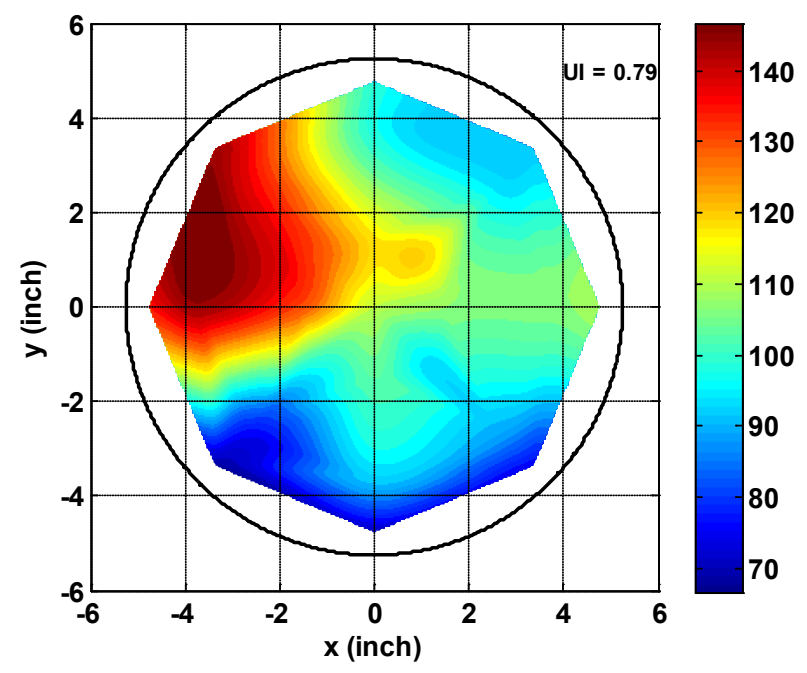

Figure 5.20: Distribution profile of $\mathrm{NH}_{3}$ with the average ANR of 0.3 for test point 1 


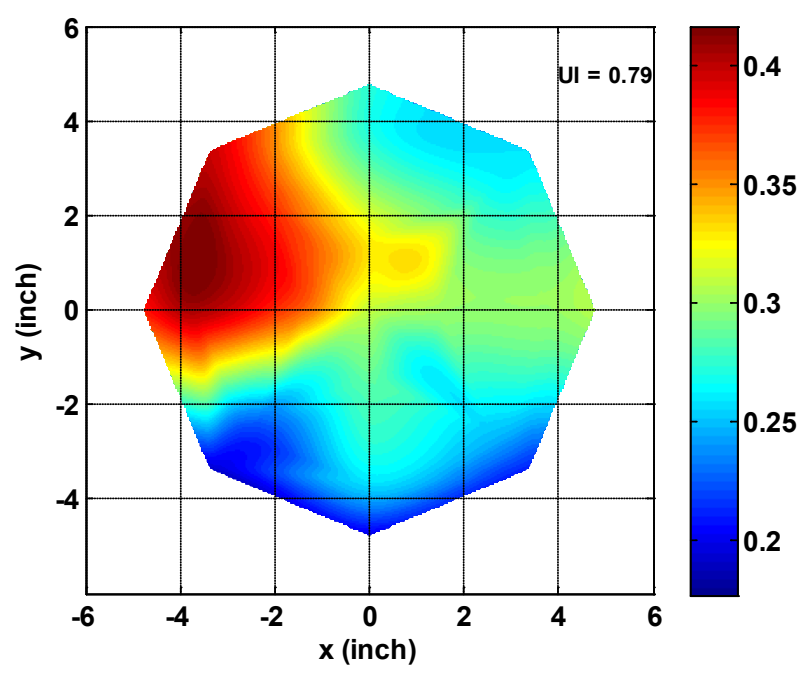

Figure 5.21: Distribution profile of ANR with the average ANR of 0.3 for test point 1

Test number 4, 5 and 6 in Table 3.13 were performed with an additional swirl mixer installed into the SCR inlet cone to further mix the injected DEF with the exhaust flow. The determined ANR distribution at the SCR inlet for test number 5 is given in Figure 5.22. Figure 5.21 shows the determined ANR distribution under the same test condition but without the swirl mixer. It can be seen that the uniformity of ANR at the SCR inlet was improved to a UI of 0.9 with the swirl mixer compared to below 0.8 without the mixer. 


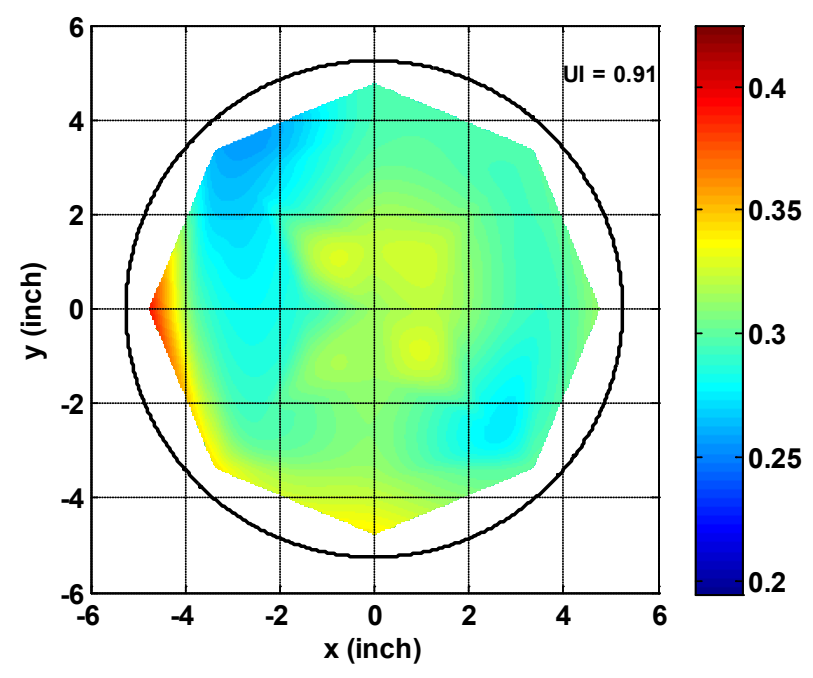

Figure 5.22: Distribution of ANR after installing swirl mixer for condition for test point 5

The $\mathrm{NH}_{3}$ maldistribution test results are summarized and shown in Table 5.6. The contour plots of the SCR inlet ANR for all test conditions are shown in Appendix $L$. It can be seen that the $\mathrm{SCR}$ inlet $\mathrm{NH}_{3}$ and the ANR distributions were not uniform $(\mathrm{UI}<0.95)$ for all test conditions. From the comparison between test number 1 and 2, it can be observed that the changes in exhaust mass flow rate slightly affects the uniformity of the SCR inlet $\mathrm{NH}_{3}$ and ANR. With the exhaust mass flow rate increased from 4.23 to 9.89 $\mathrm{kg} / \mathrm{min}$, the $\mathrm{UI}$ of the SCR inlet $\mathrm{NH}_{3}$ and $\mathrm{ANR}$ was reduced from 0.79 to 0.76 . From the comparison between test number 4 and 6 , it can be seen that the change in the SCR inlet temperature did not affect the uniformity of the SCR inlet $\mathrm{NH}_{3}$ and ANR. From the comparison between test number 2 and 3 , it can be observed that slightly higher UI (0.78 compared to 0.76) was achieved with higher DEF injection rate which gave an average ANR of 0.65 . The $\mathrm{NH}_{3}$ uniformity is improved by installing the additional swirl mixer into the SCR inlet cone. The uniformity index of the $\mathrm{NH}_{3}$ and ANR with the mixer is around 0.90 compared to below 0.80 without the mixer.

Because it is difficult to achieve different $\mathrm{NH}_{3}$ maldistribution levels in the engine test cell, and it is time and cost consuming for running engine experiments, the $\mathrm{NH}_{3}$ 
maldistribution effects on the SCR performance were determined through simulation studies as introduced in the following section.

Table 5.6: Test results for the $\mathrm{NH}_{3}$ maldistribution tests

\begin{tabular}{|c|c|c|c|c|c|c|}
\hline $\begin{array}{c}\text { Test } \\
\text { No. }\end{array}$ & $\begin{array}{c}\text { Exh } \\
\text { Mass } \\
\begin{array}{c}\text { Flow } \\
\text { Rate } \\
(\mathbf{k g} / \mathbf{m i n})\end{array}\end{array}$ & $\begin{array}{c}\text { SCR } \\
\text { Inlet } \\
\text { Temp. } \\
\left({ }^{\circ} \mathbf{C}\right)\end{array}$ & $\begin{array}{c}\text { SCR } \\
\text { Inlet } \\
\text { Average } \\
\text { ANR }\end{array}$ & $\begin{array}{c}\text { SCR } \\
\text { Inlet } \\
\text { Average } \\
\mathbf{N H}_{3} \\
(\mathbf{p p m})\end{array}$ & $\begin{array}{c}\text { Standard } \\
\text { Deviation } \\
\text { in SCR } \\
\text { inlet } \mathbf{N H}_{3} \\
(\mathbf{p p m})\end{array}$ & $\begin{array}{c}\text { Ul of } \\
\text { Determined } \\
\text { SCR inlet } \\
\mathbf{N H}_{3} \text { and } \\
\text { ANR }\end{array}$ \\
\hline 1 & 4.23 & 350 & 0.30 & 106 & 22.2 & 0.79 \\
\hline 2 & 9.89 & 350 & 0.30 & 82 & 19.6 & 0.76 \\
\hline 3 & 9.89 & 350 & 0.65 & 179 & 38.9 & 0.78 \\
\hline $4^{*}$ & 4.20 & 300 & 0.30 & 108 & 9.41 & 0.91 \\
\hline $5^{*}$ & 4.20 & 350 & 0.30 & 104 & 9.76 & 0.91 \\
\hline $6^{*}$ & 10.0 & 350 & 0.30 & 83 & 9.38 & 0.89 \\
\hline
\end{tabular}

* Test No. 4, 5 and 6 were performed with an additional swirl mixer in the SCR inlet cone.

\subsection{2 $\mathrm{NH}_{3}$ Maldistribution Effects on SCR Performance}

A series of simulation studies were carried out for different SCR conditions to understand the $\mathrm{NH}_{3}$ maldistribution effects on the SCR performance. A multi-channel model based on the high fidelity SCR model calibrated to the reactor data was setup for the simulation. The model contained 24 separate channels with different inlet ANR levels to simulate the inlet $\mathrm{NH}_{3}$ maldistribution. A schematic of the model setup is shown in Figure 5.23. As shown in the figure, the average ANR was multiplied by a maldistribution factor $A(i)$ for each individual channel. The maldistribution factors representing different Uls were generated from a normal distributions based on a mean value of 1.0 and different standard deviations. The probability distribution function of the maldistribution factors for the $\mathrm{UI}$ of $0.7,0.8$, and 0.9 is given in Figure 5.24. The average of the outlet concentrations from each channel were considered as the simulated outlet concentrations. The model also gave predictions of gaseous concentrations and $\mathrm{NH}_{3}$ storage at different axial locations of each channel. 


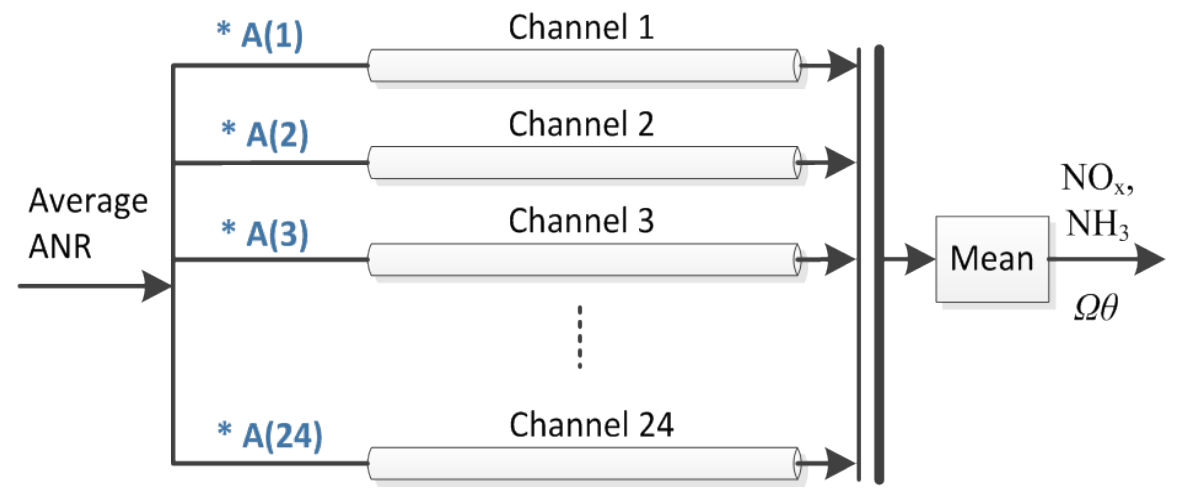

Figure 5.23: Schematic showing the setup of the model for maldistribution effects simulation

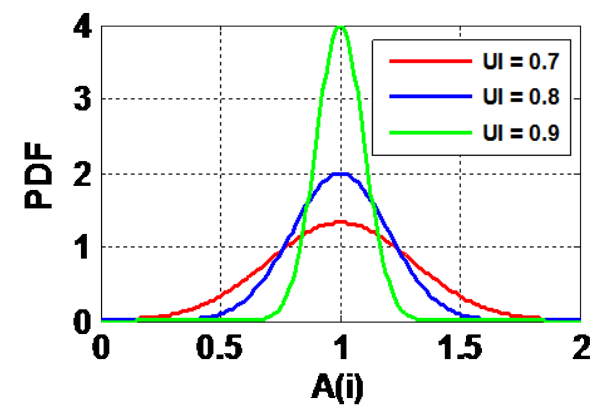

Figure 5.24: Probability distribution function of maldistribution factors for the UI of 0.7 , 0.8 and 0.9

A four-step simulation protocol for the $\mathrm{NO}_{2} / \mathrm{NO}_{x}$ ratio of 0 shown in Figure 5.25 was devised to investigate the maldistribution effects on the $\mathrm{NH}_{3}$ storage and of $\mathrm{NO}_{\mathrm{X}}$ reduction efficiency. The inlet gases also include $7.0 \% \mathrm{H}_{2} \mathrm{O}, 8.0 \% \mathrm{CO}_{2}$, and $10.0 \% \mathrm{O}_{2}$ to simulate a typical engine exhaust gas condition. The complete set of simulation conditions is given in Table 5.7. Three SCR temperatures and one space velocity of $60,000 \mathrm{hr}^{-1}$ with $\mathrm{NO}_{2} / \mathrm{NO}_{X}$ ratio of $0,0.5$ and 1.0 were simulated. For each simulation condition, the average inlet ANR was changed from 0.6 to 1.2 with a interval of 0.2 . For each average ANR of each condition, three different maldistribution levels with UI of 0.7 , $0.8,0.9$ and a uniform condition $(\mathrm{UI}=1.0)$ were simulated. 


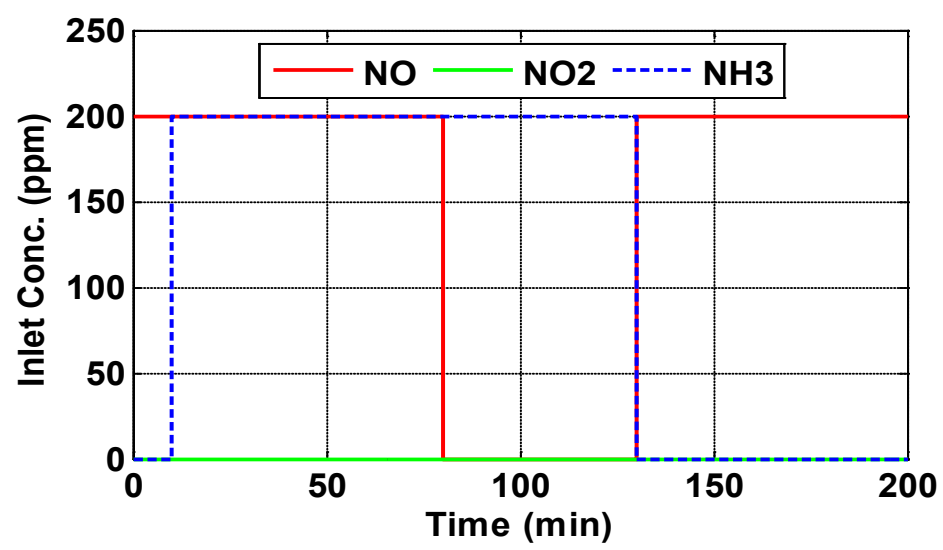

Figure 5.25: Setup of the SCR inlet concentrations for simulation

Table 5.7: Simulation conditions for maldistribution effect study

\begin{tabular}{|c|c|c|c|c|}
\hline $\begin{array}{c}\text { Space } \\
\text { Velocity }\left(\mathrm{hr}^{-1}\right)\end{array}$ & $\begin{array}{c}\mathbf{S C R} \\
\left.\text { Temp. }^{\circ} \mathbf{C}\right)\end{array}$ & $\begin{array}{c}\mathbf{N O}_{2} / \mathbf{N O}_{\mathbf{x}} \\
\text { Ratio }\end{array}$ & $\begin{array}{c}\text { Average } \\
\text { ANR }\end{array}$ & UI \\
\hline 60,000 & $250,300,350$ & $0,0.5,1.0$ & $0.6,0.8,1.0,1.2$ & $0.7,0.8,0.9,1.0$ \\
\hline
\end{tabular}

The simulated $\mathrm{NO}_{x}$ reduction efficiencies and $\mathrm{NH}_{3}$ slip as a function of the average ANR for different Uls under the SCR temperature of $300^{\circ} \mathrm{C}$ and $\mathrm{NO}_{2} / \mathrm{NO}_{X}$ ratio of 0.0 is shown in Figures 5.26 and 5.27. It can be observed that the decrease in UI reduced the $\mathrm{NO}_{X}$ reduction and increased the $\mathrm{NH}_{3}$ slip when the average inlet ANR is above 0.6. When changing the $\mathrm{UI}$ from 0.7 to 1.0 with an average of 1.0 , the $\mathrm{NO}_{x}$ reduction efficiency was increased by $10 \%$ and the $\mathrm{NH}_{3}$ slip was reduced by $15 \mathrm{ppm}$. The SCR performance with Uls of 0.9 and 1.0 is close. The UI effects were similar for the average ANR of 1.0 and 1.2 and were less obvious with lower average ANRs. This is because all of the $\mathrm{NH}_{3}$ participates with the $\mathrm{NO}_{X}$ reduction reactions when the average ANR is low. When the average ANR is close to 1.0, the inlet ANR of certain channels might get higher than 1.0. The excessive $\mathrm{NH}_{3}$ left after reacting with $\mathrm{NO}_{x}$ exits the channel as $\mathrm{NH}_{3}$ slip. The $\mathrm{NH}_{3}$ storage was not significantly affected by the different values of UI. 


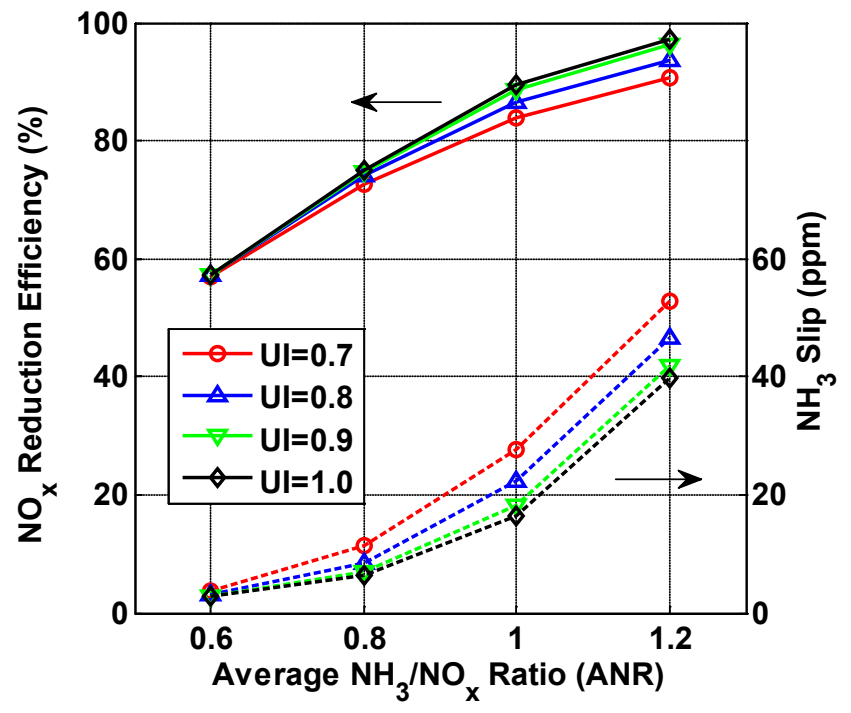

Figure 5.26: $\mathrm{NO}_{\mathrm{x}}$ reduction efficiency and $\mathrm{NH}_{3}$ slip as a function of average ANR for different UI

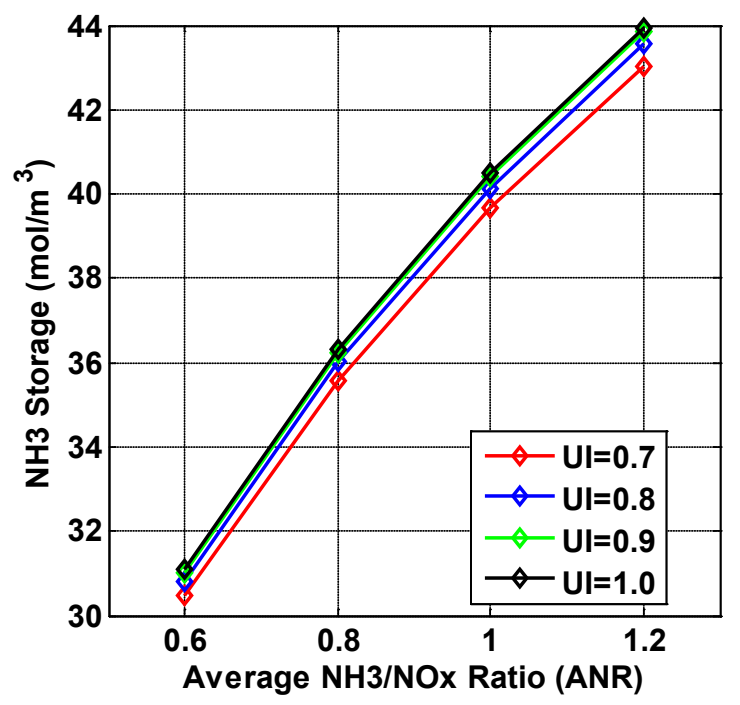

Figure 5.27: $\mathrm{NH}_{3}$ storage as a function of average ANR for different UI

Similar effects were observed in the other simulation runs. The $\mathrm{NO}_{\mathrm{x}}$ reduction was reduced by $5-10 \%$ with 10 to 20 ppm increase in $\mathrm{NH}_{3}$ slip for the different conditions. 
The $\mathrm{NH}_{3}$ slip can be oxidized with the ammonia oxidation catalyst (AMOX) in the production aftertreatment system. However, reducing the SCR outlet $\mathrm{NH}_{3}$ through optimal mixing helps reduce the DEF consumption. Smaller maldistribution effects were observed with a $\mathrm{NO}_{2} / \mathrm{NO}_{\mathrm{x}}$ ratio of 1.0 . This is probably because the $\mathrm{NO}_{\mathrm{x}}$ reduction is kinetic limited under this situation because of the low reaction rates of the slow SCR reaction mechanisms.

\subsubsection{Maldistribution Effects on SCR Model Parameters}

As discussed above, there were differences in the SCR model parameters when calibrated to reactor versus engine test cell data as shown in Table 5.1. In order to determine if the $\mathrm{NH}_{3}$ maldistribution is a factor causing the differences, a simulation study of the engine experimental data was carried out by running the reactor data based model with multiple channels and nonuniform inlet $\mathrm{NH}_{3}$.

A comparison of the SCR model simulation from a single channel model calibrated to the engine data compared to the steady state SCR engine test results is shown in Figure 5.9. The test condition for this run was $1720 \mathrm{rpm}, 503 \mathrm{~N} \cdot \mathrm{m}$ with a SCR inlet temperature of $376^{\circ} \mathrm{C}$. The test was performed before the additional swirl mixer was installed. It can be seen that the model is able to follow the time histories of the SCR outlet $\mathrm{NO}, \mathrm{NO}_{2}$, and $\mathrm{NH}_{3}$. Because of the different model parameters in the SCR model calibrated to reactor data, simulation results of the same set of engine experimental data using a single channel reactor data based model showed obvious disagreements with the data. The comparison of SCR reactor data based model simulation results with engine data is given in Figure 5.28. It can be seen that the model simulated results show higher $\mathrm{NO}_{\mathrm{x}}$ conversion efficiency and lower $\mathrm{NH}_{3}$ slip compared to the experimental data during the DEF injection periods. It should be noted that the sample line model was incorporated in order to compare the simulated $\mathrm{NH}_{3}$ slip with the IMR-MS measurements. 


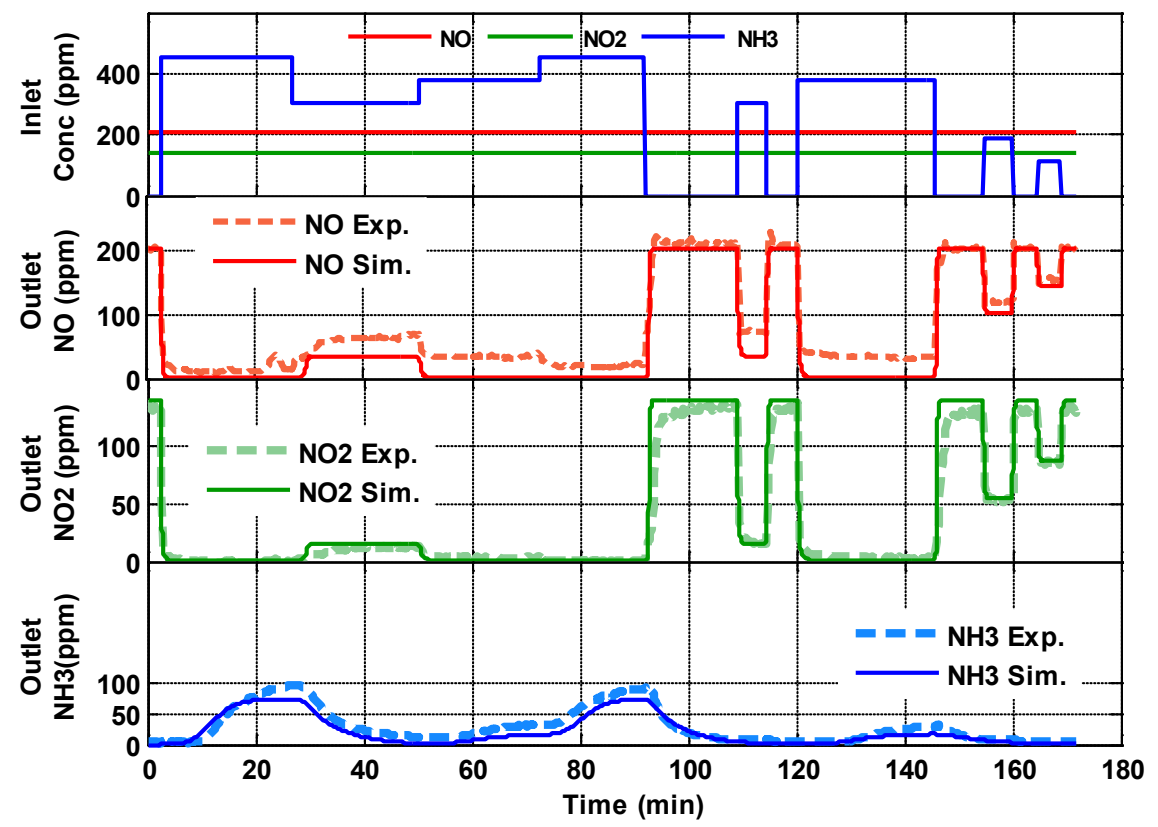

Figure 5.28: Single channel SCR model simulation results with calibration to reactor data compared to engine experimental data

In order to take the $\mathrm{NH}_{3}$ maldistribution effect into account when running the simulation, the multi-channel model was setup with model parameters developed from the reactor data to simulate the engine experimental data. The model also contained 24 channels with a different inlet $\mathrm{NH}_{3}$ concentration for each channel. The inlet $\mathrm{NH}_{3}$ concentration of each channel was assigned by applying a maldistribution factor generated based on the maldistribution test results. A contour plot of the normalized maldistribution factors applied to each channel is shown in Figure 5.29. The set of maldistribution factors has a mean value of 1.0 and $\mathrm{UI}$ of 0.79 which is the same as the maldistribution test results. 


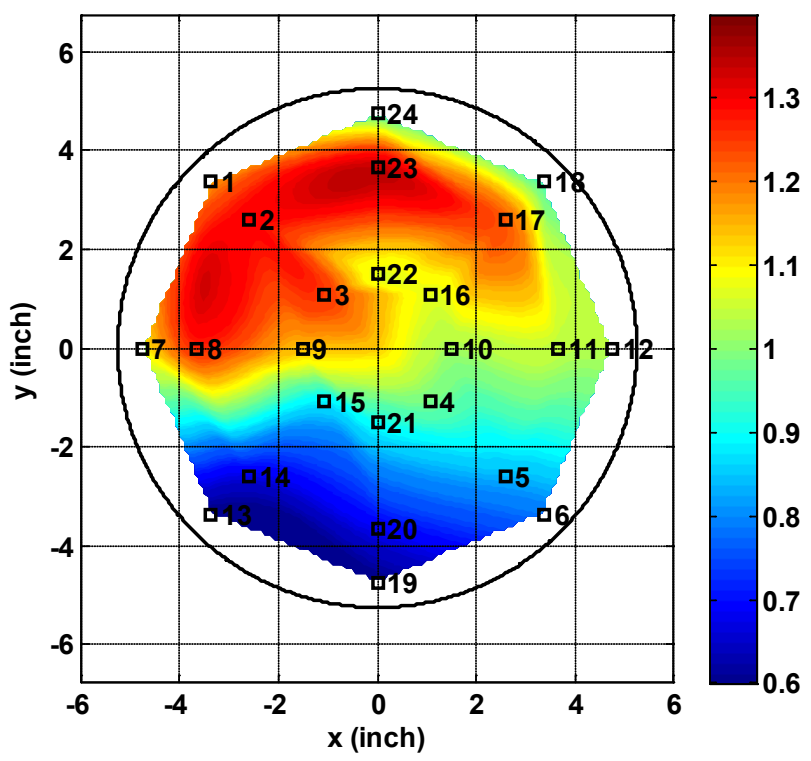

Figure 5.29: Contour plot of the maldistribution factors applied to each channel of the 24channel model with parameters developed from the reactor data

The average of outlet concentrations from each channel is considered as the model simulated outlet concentrations. The multi-channel model simulation results compared to the engine experimental data is shown in Figure 5.30. It can be observed that the simulation results still showed lower outlet $\mathrm{NO}$ and higher $\mathrm{NO}_{2}$ concentrations. The simulated $\mathrm{NH}_{3}$ slip post processed by the sample line model follows the time history of the engine experimental data. The cost functions values for $\mathrm{NO}, \mathrm{NO}_{2}$, and $\mathrm{NH}_{3}$ for the simulation results from both the single and multi-channel models are given in Table 5.8. Lower overall cost function values were achieved by considering the $\mathrm{NH}_{3}$ maldistribution with the multi - channel model. Compared to the single model simulation results with model parameters based on reactor data shown in Figure 5.28, the multi-channel model with same model parameters gave simulation results which are closer to the engine experimental data by taking maldistribution effects into consideration. The conclusion is that the maldistribution effect is a factor causing the different model parameters between the SCR model calibrated to reactor and engine experimental data. The SCR model calibrated to the engine data takes the complex engine exhaust conditions into account and is able to simulate the SCR system with those effects. 


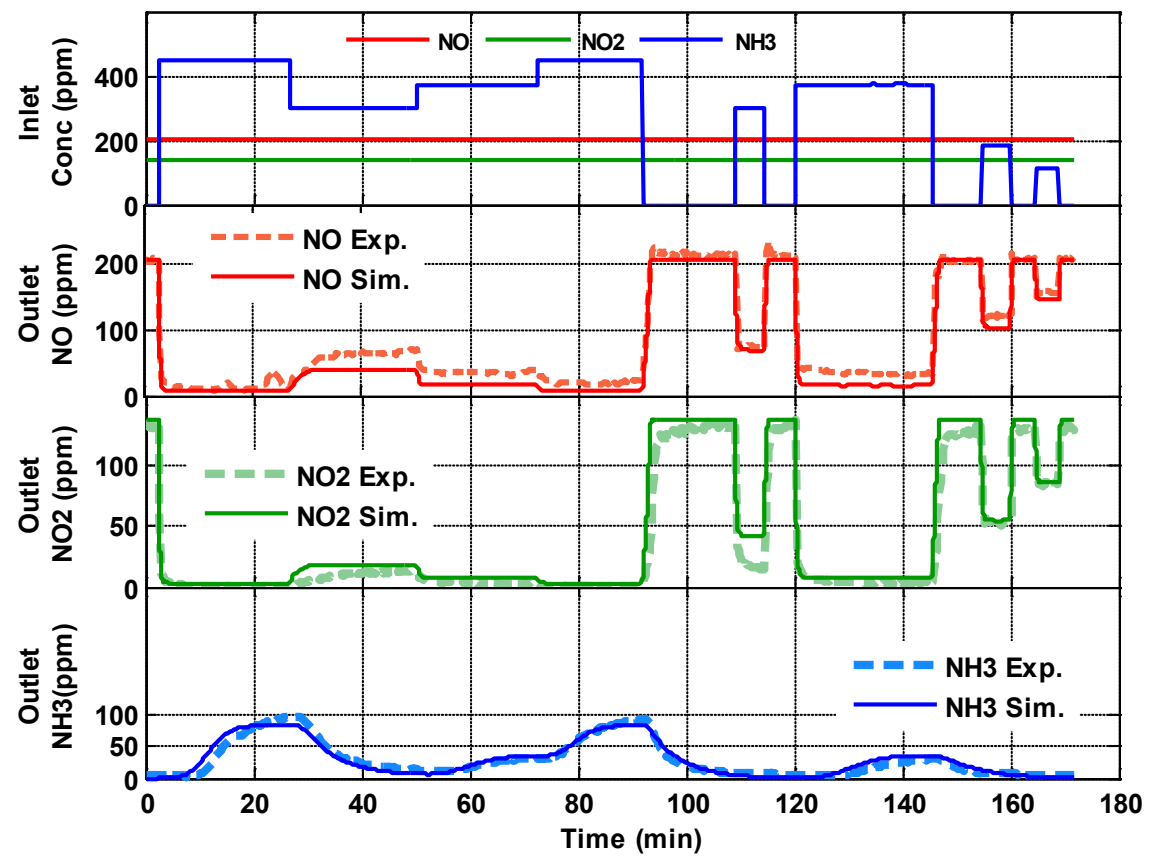

Figure 5.30: Multi-channel model simulation results compared to engine experimental data

Table 5.8: Cost values for simulation results from the single and multi-channel models

\begin{tabular}{|c|c|c|c|c|}
\hline & $\mathbf{N O}$ & $\mathbf{N O}_{2}$ & $\mathbf{N H}_{3}$ & Total \\
\hline Single Channel Model & 21.9 & 5.52 & 8.90 & 36.3 \\
\hline Multi Channel Model & 14.1 & 7.15 & 5.50 & 26.8 \\
\hline
\end{tabular}

\subsection{SCR Thermal Aging Data and Effects}

The representative published experimental studies of the thermal aging effects on the SCR catalyst have been introduced in Section 2.6. It was concluded that the thermal aging deactivates the $\mathrm{NO}_{x}$ reduction performance of the SCR and reduces the $\mathrm{NH}_{3}$ storage capacity. However, no modeling or kinetics study of the thermal aging effects has been published. In this study, both a de-greened and hydrothermally aged Cuzeolite SCR catalyst were tested with the improved Spaci-IR technique developed at ORNL to reveal the thermal aging effects on the axially resolved concentration and 
storage profiles. The experimental data also provided opportunities for analyzing the aging effects on the SCR kinetics from the modeling aspect.

In order to prevent undesired high local space velocity observed from previous Spaci-IR experiments, several improvements were made for the thermal aging testing. Those improvements include (1) four capillaries with bigger diameters $(0.65 \mathrm{~mm}$ outer diameter) were used to sample from four different SCR channels, (2) longer core SCR sample so that there is higher exhaust mass flow rate going into each channel for a given space velocity, (3) Higher SCR inlet $\mathrm{NO}_{x}$ and $\mathrm{NH}_{3}$ concentrations to increase measurable concentration levels at different axial locations and (4) lower reactor pressure [133].

Both the de-greened and aged Cu-zeolite SCR core samples were $8 \mathrm{~cm}$ in length and 2 $\mathrm{cm}$ in diameter. The de-green condition was $700^{\circ} \mathrm{C}$ for four hours with $20 \% \mathrm{O}_{2}, 4.5 \%$ $\mathrm{H}_{2} \mathrm{O}$ and balance $\mathrm{N}_{2}$ while the aging condition was $800^{\circ} \mathrm{C}$ for 16 hours under same gas compositions to simulate $220,000 \mathrm{~km}$ of vehicle aging [115]. The samples were tested under three temperatures of 250,300 and $350^{\circ} \mathrm{C}$ and a constant space velocity of $40,000 \mathrm{hr}^{-1}$. A simplified eight step test protocol shown in Figure 5.31 was conducted with concentrations being measured at nine different axial locations of the catalyst channel for each temperature. The detailed information about each step of the protocol is shown in Table 5.9. The experimental data were used to quantify the axial concentration and $\mathrm{NH}_{3}$ storage profiles for both the de-greened and aged catalysts. The data process procedures are similar to the procedures introduced in Appendix F. 


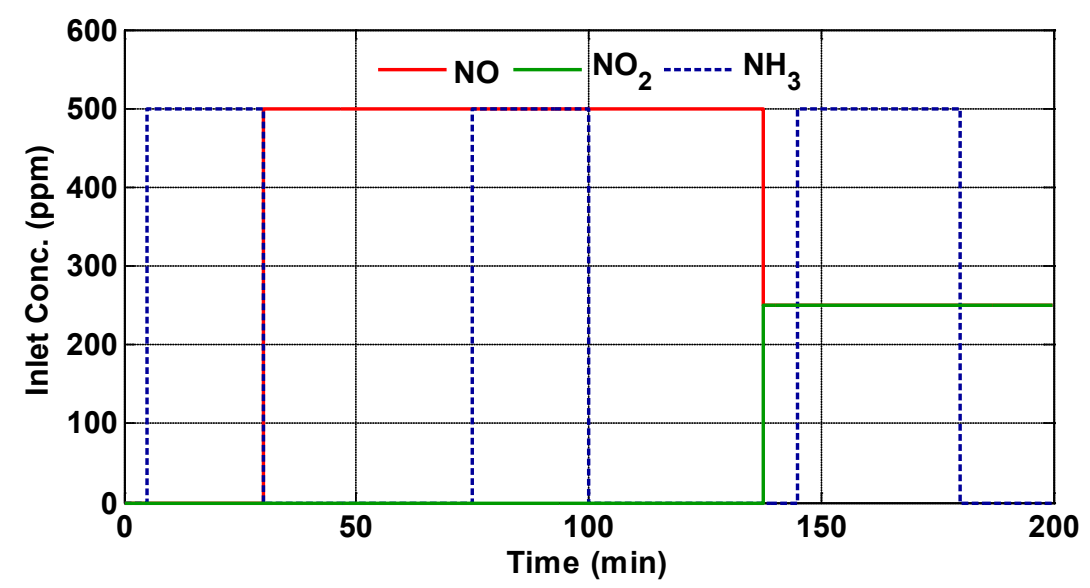

Figure 5.31: Test protocol for SCR hydrothermal aging tests

Table 5.9: Details for each step of the Spaci-IR aging test protocol

\begin{tabular}{|c|c|c|c|c|}
\hline Step & Description & NO (ppm) & $\mathrm{NO}_{2}$ (ppm) & $\mathrm{NH}_{3}(\mathrm{ppm})$ \\
\hline 1 & Stabilize & 0 & 0 & 0 \\
\hline 2 & $\mathrm{NH}_{3}$ saturation & 0 & 0 & 500 \\
\hline 3 & $\mathrm{NH}_{3}$ inventory, $\quad \mathrm{NO}_{2} / \mathrm{NO}_{\mathrm{x}}=0$ & 500 & 0 & 0 \\
\hline 4 & $\mathrm{NH}_{3} / \mathrm{NO}_{\mathrm{x}}=1.0 ; \quad \mathrm{NO}_{2} / \mathrm{NO}_{\mathrm{x}}=0$ & 500 & 0 & 500 \\
\hline 5 & $\mathrm{NH}_{3}$ inventory: $\quad \mathrm{NO}_{2} / \mathrm{NO}_{\mathrm{x}}=0$ & 500 & 0 & 0 \\
\hline 6 & Stabilize $\mathrm{NO}, \quad \mathrm{NO}_{2} / \mathrm{NO}_{\mathrm{x}}=0.5$ & 250 & 250 & 0 \\
\hline 7 & $\mathrm{NH}_{3} / \mathrm{NO}_{\mathrm{x}}=1.0 ; \mathrm{NO}_{2} / \mathrm{NO}_{\mathrm{x}}=0.5$ & 250 & 250 & 500 \\
\hline 8 & $\mathrm{NH}_{3}$ inventory, $\quad \mathrm{NO}_{2} / \mathrm{NO}_{\mathrm{x}}=0.5$ & 250 & 250 & 0 \\
\hline
\end{tabular}

A comparison of the cumulative $\mathrm{NH}_{3}$ stored on the catalyst at the equilibrium state of steps 2, 4 and 7 as functions of fractional axial position under different temperatures are shown in Figure 5.32. For both aging conditions, it can be seen that the $\mathrm{NH}_{3}$ stored decreases under all conditions with increase in temperature. Step 2 of the protocol is the $\mathrm{NH}_{3}$ saturation condition with highest storage levels. The cumulative $\mathrm{NH}_{3}$ stored increases linearly with the axial length of the catalyst channel. The last measurement point near the end of the channel showed a different trend because of the heavier washcoat loading on the substrate. Compared to the de-greened catalyst, the $\mathrm{NH}_{3}$ stored on the aged catalyst is about $30 \%$ lower at all three temperatures. This is because the thermal aging "reduces the number and/or the stability of the $\mathrm{NH}_{3}$ storage sites" [133]. Step 4 is the standard SCR condition with a $\mathrm{NO}_{2} / \mathrm{NO}_{\mathrm{x}}$ ratio of 0 and ANR of 
1. The stored $\mathrm{NH}_{3}$ kept increasing from the inlet to outlet under the temperature of $250^{\circ} \mathrm{C}$ with a lowered increasing rate towards the end of the catalyst channel. This is because the $\mathrm{NH}_{3}$ concentration decreases along the catalyst length and the storage is a function of the $\mathrm{NH}_{3}$ concentration. The stored $\mathrm{NH}_{3}$ stopped increasing from 0.4 of the catalyst length under the temperature of $350^{\circ} \mathrm{C}$, meaning that there is no $\mathrm{NH}_{3}$ storage after the 0.4 axial location. The reason is that the $\mathrm{NH}_{3}$ is completely converted in the first 0.4 catalyst length. The difference in the $\mathrm{NH}_{3}$ stored between this condition and the $\mathrm{NH}_{3}$ saturation condition were consumed by the reactions between $\mathrm{NO}$ and the $\mathrm{NH}_{3}$. The $\mathrm{NH}_{3}$ stored on the aged catalyst is lower than the de-greened catalyst but the smaller differences were observed compared to the $\mathrm{NH}_{3}$ saturation condition (Step 2). Step 4 is the fast SCR condition with a $\mathrm{NO}_{2} / \mathrm{NO}_{x}$ ratio of 0.5 and ANR of 1 . It can be seen that the $\mathrm{NH}_{3}$ stored on the catalyst is about $50 \%$ less compared to the standard SCR reaction under all three temperatures. This is because the fast SCR reaction with a much higher reaction rate is dominant under this condition and consumes more stored $\mathrm{NH}_{3}$, resulting in less $\mathrm{NH}_{3}$ storage at the equilibrium state compared to the standard SCR reaction. Similarly, the cumulative $\mathrm{NH}_{3}$ stored stopped increasing after 0.4 of the catalyst length under all three temperatures, meaning that the $\mathrm{NH}_{3}$ persisted shorter after entering the SCR than the standard SCR reaction. The differences in the $\mathrm{NH}_{3}$ stored between the degreened and aged catalysts are not obvious at step 4 except the first 4 measurement locations under 250 and $300^{\circ} \mathrm{C}$. The reason for this is a combination of the decrease in the $\mathrm{NH}_{3}$ storage capability and the different axial concentration profiles. 

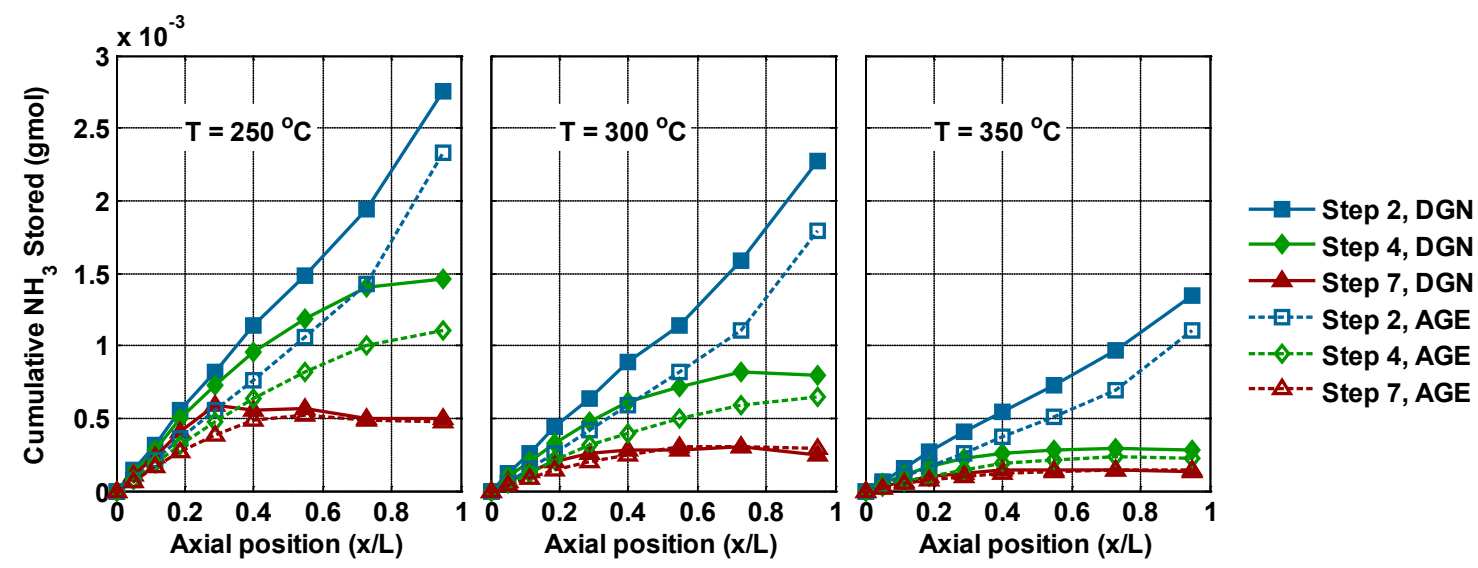

Figure 5.32: Comparison of Cumulative $\mathrm{NH}_{3}$ stored between de-greened and aged catalysts

The axial $\mathrm{NO}_{x}$ and $\mathrm{NH}_{3}$ concentration distribution profiles under the temperature $350^{\circ} \mathrm{C}$ for both de-greened and aged catalysts are shown in Figure 5.33. It can be seen the $\mathrm{NH}_{3}$ concentrations at the last measurement position are zero for both aging conditions. The aging effect on the SCR outlet $\mathrm{NO}_{x}$ concentrations is small with a difference less than 8 $\mathrm{ppm}$ at the last axial measurement position. That means that the hydrothermal aging does not significantly change the overall conversion efficiencies for $\mathrm{NO}_{x}$ and $\mathrm{NH}_{3}$ under the space velocity of 40,000 $\mathrm{hr}^{-1}$. Obvious differences in axial $\mathrm{NO}_{x}$ and $\mathrm{NH}_{3}$ concentration profiles were observed internal the SCR channel. Compared to the degreened catalyst, the concentration profiles for the aged catalyst were shifted towards the outlet direction of the catalyst, meaning relatively higher $\mathrm{NO}_{x}$ and $\mathrm{NH}_{3}$ concentrations at different axial locations and slower $\mathrm{NO}_{x}$ and $\mathrm{NH}_{3}$ conversion rates. The reduced $\mathrm{NH}_{3}$ storage capability along with the different axial concentration profiles resulted in the differences and similarities in stored $\mathrm{NH}_{3}$ at steps 4 and 7 shown in Figure 5.32. From the $\mathrm{NO}_{x}$ and $\mathrm{NH}_{3}$ concentration profiles at step 7 shown in Figure 5.33, it can be observed that the $\mathrm{NH}_{3}$ concentration agrees with the $\mathrm{NO}_{x}$ concentration for the degreened catalyst. The $\mathrm{NH}_{3}$ concentration is slightly lower than the $\mathrm{NO}_{x}$ concentration for the aged catalyst, indicating that there is a slight $\mathrm{NH}_{3}$ overconsumption. This could be explained by a relatively higher $\mathrm{NH}_{3}$ oxidation reaction rate for the aged catalyst under this temperature. It was reported in reference $[44,114]$ that hydrothermal aging under $850^{\circ} \mathrm{C}$ for 10 hours may lead to an increase in the $\mathrm{NH}_{3}$ oxidation reaction and the oxidation selectivity to $\mathrm{NO}_{x}$. The slower conversion rate of $\mathrm{NO}_{\mathrm{x}}$ and $\mathrm{NH}_{3}$ and the different 
concentration profiles observed from the aged catalyst can be explained by lowered reaction rate caused by thermal aging. This could be due to a reduction in $\mathrm{NH}_{3}$ storage capability or a decrease in reaction rate constant cause by deactivation of the catalytic material or both. However, "it's not clear if these two impacts are independent or directly related" [133]. In order to answer this question, a modeling study was conducted to further study the characteristics of the catalysts with different aging conditions and explain the hydrothermal aging effects on the kinetics of the SCR.
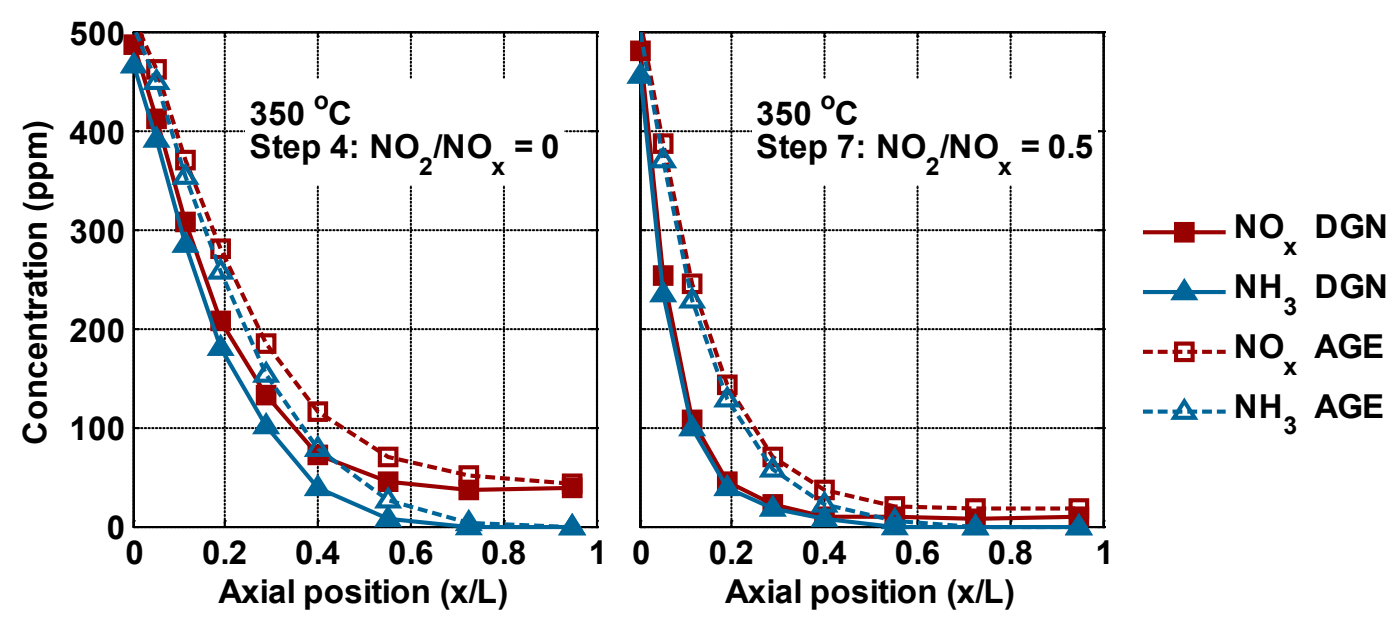

Figure 5.33: Axial $\mathrm{NO}_{x}$ and $\mathrm{NH}_{3}$ distribution profiles at steps 4 and 7 under the temperature of $350^{\circ} \mathrm{C}$

A modeling procedure based on the Spaci-IR data was introduced in Section 4.2. A similar procedure was carried out for both de-greened and aged catalysts to determine the aging effects on the kinetic parameters from the modeling perspective. For the purpose of simplifying the calculation, a 1-site modeling approach was used to compute the total ammonia storage. The first step was to determine the storage capacity of the catalysts. The $\mathrm{NH}_{3}$ storage as a function of temperature for both de-greened and aged catalysts are shown in Figure 5.34. Based on the $\mathrm{NH}_{3}$ storage and Equation 4.16 which was previously derived from the model equations, the total storage capacity of the catalysts $(\Omega)$, and the relationships of the kinetic parameters between the $\mathrm{NH}_{3}$ adsorption and desorption reactions (Aads/Ades, Eads-Edes) can be calculated. The results for both catalysts are shown in Table 5.10. Zero activation energy was applied to the adsorption reaction because it was typically considered as a non-activated reaction. 
It can be seen through the comparison in Table 5.10 that the storage capacity of the catalyst is reduced by $30 \%$ after the hydrothermal aging. The lowered desorption reaction activation energy and a different Aads/Ades ratio indicates that the adsorption and desorption properties of the catalyst are also changed.

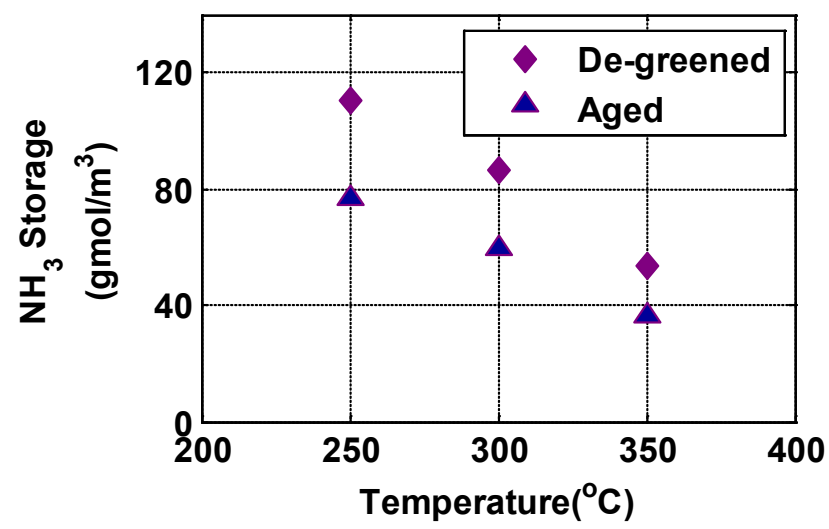

Figure 5.34: $\mathrm{NH}_{3}$ storage as a function of temperature for de-greened and aged catalysts

Table 5.10: Details for each step of the Spaci-IR aging test protocol

\begin{tabular}{|c|r|r|c|}
\hline Parameter & De-greened & Aged & Unit \\
\hline$\Omega$ & 138 & 94.6 & $\mathrm{gmol} / \mathrm{m}^{3}$ \\
\hline Aads / Ades & $4.29 \mathrm{e}-2$ & $3.67 \mathrm{e}-1$ & none \\
\hline Eads & 0 & 0 & $\mathrm{~kJ} / \mathrm{gmol}$ \\
\hline Edes & 38.2 & 28.6 & $\mathrm{~kJ} / \mathrm{gmol}$ \\
\hline
\end{tabular}

A comparison of cumulative $\mathrm{NH}_{3}$ stored under the $\mathrm{NH}_{3}$ saturation condition (step 2) between the experimental and model simulation results is shown in Figure 5.35. It can be seen that the model is able to simulate the $\mathrm{NH}_{3}$ storage under different temperatures for both de-greened and aged catalysts. The last axial measurement point does not agree with the simulation results because the heavier washcoat loading at the end of the core catalyst sample (due to the catalyst manufacturing process) was not considered in the model. 

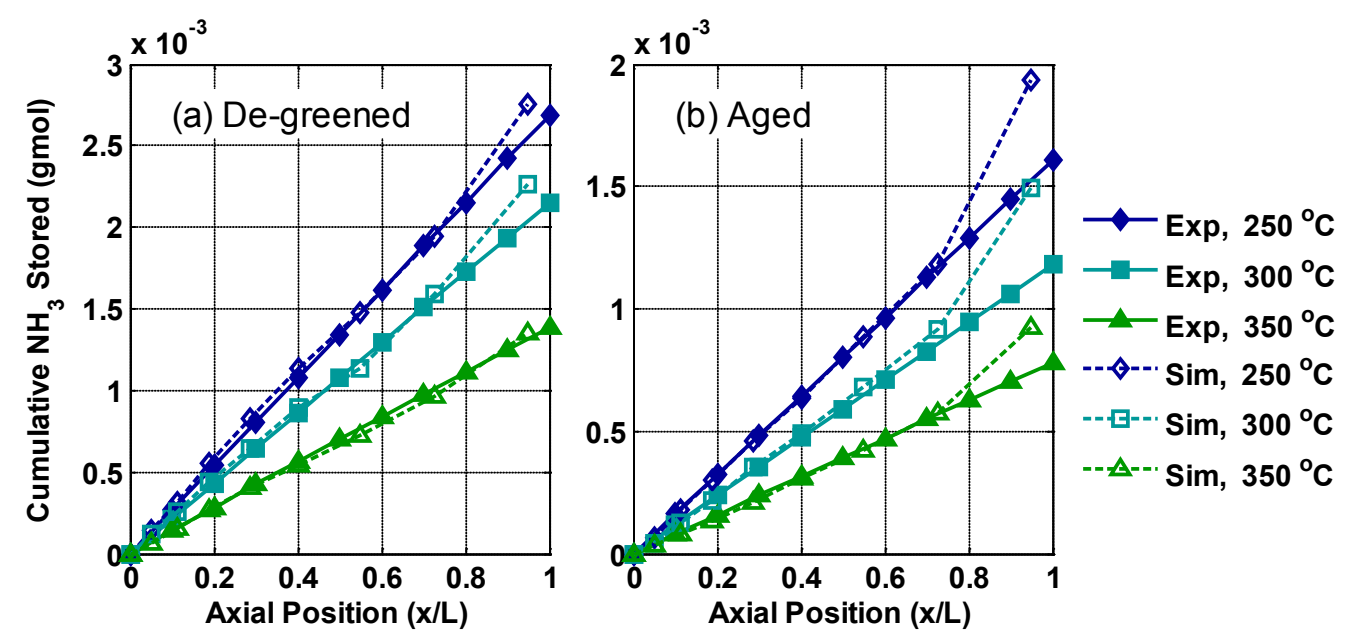

Figure 5.35: Experimental and simulated cumulative $\mathrm{NH}_{3}$ stored under $\mathrm{NH}_{3}$ saturation condition for both de-greened and aged catalysts

The kinetics for the $\mathrm{NH}_{3}$ oxidation, standard SCR and fast SCR reactions were then analyzed based on the model equations and the experimental results from steps 2, 4 and 7 of the test protocol. The $\mathrm{NH}_{3}$ adsorption, desorption and oxidation reactions take place at step 2 of the test protocol. The equilibrium state depends on the reaction rates of the three reactions as shown in Equation 4.17. The axial gradients in $\mathrm{NH}_{3}$ concentration is caused by the $\mathrm{NH}_{3}$ oxidation reaction as shown in Equation 4.19. Along with the storage parameters identified above, the kinetic parameters for the $\mathrm{NH}_{3}$ oxidation reaction were quantified. Here it was assumed that the oxidation is selective to $\mathrm{N}_{2}$. A comparison in the determined pre-exponential factor and activation energy for the $\mathrm{NH}_{3}$ oxidation reaction between de-greened and aged catalysts is shown in Table 5.11. Compared to the de-greened catalyst, the thermal aging caused about $20 \mathrm{~kJ} / \mathrm{gmol}$ decrease in the activation energy and more than one magnitude decrease in the preexponential factor. A visualized comparison of the reaction rate constants in Arrhenius form is shown in Figure 5.36. It can be seen that the decrease in pre-exponential factor and the activation energy resulted in higher reaction rate constants for the aged catalyst for the temperatures studied. This agrees with the findings in reference [44,114]. 
Table 5.11: Kinetic parameters for $\mathrm{NH}_{3}$ oxidation reaction

\begin{tabular}{|c|c|c|}
\hline & Aoxi (1/s) & Eoxi (kJ/gmol) \\
\hline De-greened & $1.35 \mathrm{e} 4$ & 86.0 \\
\hline Aged & $5.81 \mathrm{e} 2$ & 66.3 \\
\hline
\end{tabular}

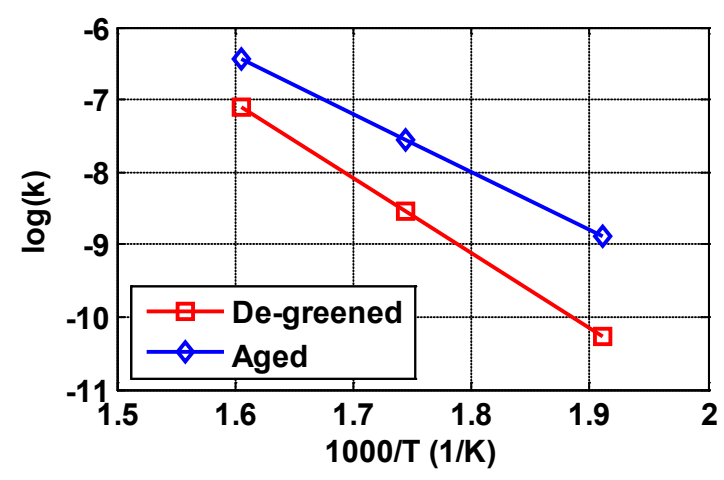

Figure 5.36: Arrhenius form plot of the $\mathrm{NH}_{3}$ oxidation reaction rate constants

A similar analysis was carried out to compare the kinetics of the standard and fast SCR reactions based on the experimental data at steps 4 and 7 of the test protocol. The standard and fast SCR reactions are dominant at steps 4 and 7 respectively and the assumption that only the dominant reaction takes place at certain steps was made to exclude the effects of unimportant reactions and simplify the analysis. The axial concentration gradient as a function of the reaction rates is given in Equation 4.19. The reaction rate equations for the standard and fast SCR reactions can be found in Table 4.1. The parameters in the equations except the reaction rate constants can be computed from the experimental data. The parameters include flow velocity, axial concentration gradients, local gaseous concentration and local $\mathrm{NH}_{3}$ storage, leaving the reaction rate constant to be solved. As a result, the reaction rate constants for the standard and fast SCR reactions on each axial element defined by the two measurement points can be determined by solving Equation 4.19 and the reaction rate equations. Compared to the $\mathrm{NH}_{3}$ concentration profile at step 2, the axial $\mathrm{NO}_{\mathrm{x}}$ and $\mathrm{NH}_{3}$ gradients at steps 4 and 7 are much larger because of the reaction rates of the standard and fast SCR reactions are higher than the $\mathrm{NH}_{3}$ oxidation reaction. The $\mathrm{NO}_{x}$ and $\mathrm{NH}_{3}$ concentrations as well as the concentration gradients dropped close to zero at the rear 
half of the catalyst channel. Slight scatter in experimental measurements may cause big errors in the reaction rate constant calculation results. As a result, only the first three axial elements with significant gaseous concentration and gradient levels were considered in the analysis. After the calculations, the averaged reaction rate constants of the first three elements were plotted in Arrhenius form for both standard and fast SCR reactions as shown in Figure 5.37. The pre-exponential factor and the activation energy for each reaction were calculated based on the Arrhenius plot and are given in Table 5.12. It can be observed that the hydrothermal aging has effects on both the standard and the fast SCR reactions by reducing the activation energy as well as the preexponential factor. The effect on the fast SCR reaction is more obvious since the reaction rate constant decreases by about one magnitude. The effect becomes more obvious at $350^{\circ} \mathrm{C}$ because of the different activation energies. The effect on the standard $\mathrm{SCR}$ reaction is not obvious as compared to the fast SCR reaction.

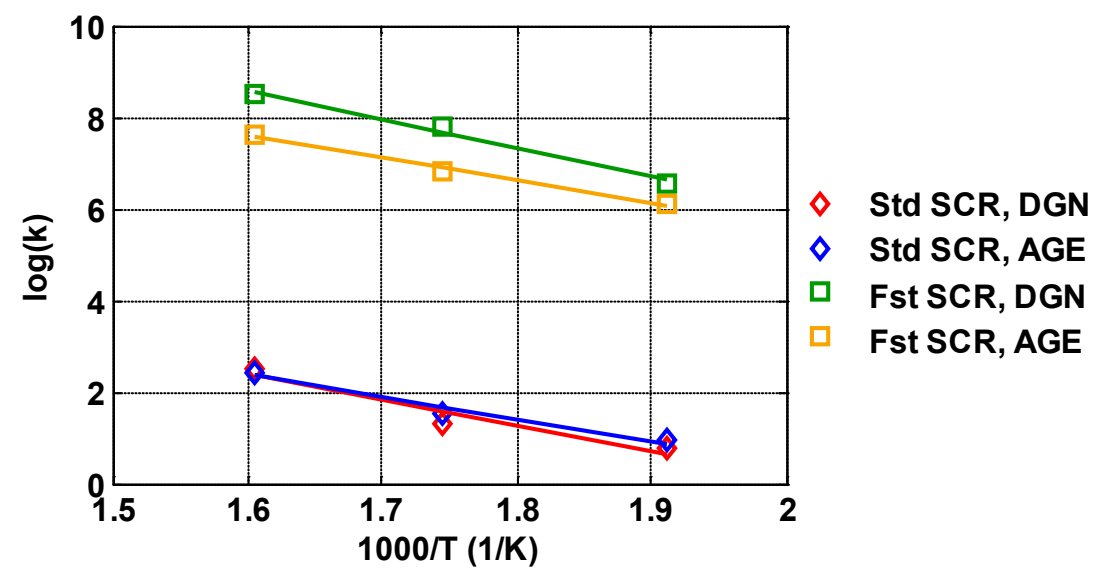

Figure 5.37: Arrhenius form plot of the reaction rate constants for standard and fast SCR reactions

Table 5.12: Kinetic parameters for the standard and fast SCR reactions

\begin{tabular}{|c|r|r|r|}
\hline Parameter & De-greened & Aged & Units \\
\hline Afst & $1.40 \mathrm{e} 8$ & $6.13 \mathrm{e} 6$ & $\mathrm{~m}^{6} / \mathrm{gmol}^{2} \cdot \mathrm{s}$ \\
\hline Efst & 52.8 & 41.6 & $\mathrm{~kJ} / \mathrm{gmol}$ \\
\hline Astd & $9.99 \mathrm{e} 4$ & $2.33 \mathrm{e} 4$ & $\mathrm{~m}^{3} / \mathrm{gmol} \cdot \mathrm{s}$ \\
\hline Estd & 47.3 & 39.9 & $\mathrm{~kJ} / \mathrm{gmol}$ \\
\hline
\end{tabular}


To summarize, the hydrothermal aging condition of $800^{\circ} \mathrm{C}$ for 16 hours has effects on both the $\mathrm{NH}_{3}$ storage capability of the catalyst and the kinetic parameters of the major SCR reactions. Both factors contributed to the change in the axial storage and concentration profiles observed from the experiments. It should be noted that the analysis was based on a series of assumptions and simplified calculations with the purpose of revealing the changes in the kinetic parameters caused by different aging conditions. 


\section{Chapter 6.}

\section{Summary and Conclusions}

The goal of this research was to develop a high fidelity SCR model that simulates the $\mathrm{NO}_{x}$ conversion, $\mathrm{NH}_{3}$ slip and $\mathrm{NH}_{3}$ storage and determine the critical kinetic parameters controlling the dynamics of the SCR system performance under varying temperatures, space velocities, exhaust inlet species, inlet ammonia maldistribution levels as well as hydrothermal aging with controlled DEF injection for a Cu-zeolite SCR catalyst and quantify their impacts on ammonia storage and $\mathrm{NO}_{\mathrm{x}}$ conversion. A further goal that evolved from this research was to explain why the kinetic parameters of the model calibrated to the reactor data were different than the parameters calibrated to the engine data. The goals have been met through coupled experimental and modeling studies. The purpose of the model development and the engine data has been to support the development of a reduced order SCR estimator model. The accomplishments and the findings from the study as well as the recommendations for the future work are given in this chapter.

\subsection{Summary}

A high fidelity multi-step global kinetic 1D 2-site SCR model with mass transfer, heat transfer and SCR chemical reactions was developed for a Cu-zeolite catalyst. The model simulates the axially resolved gaseous concentrations in the gas and surface phases, gas and wall temperatures as well as the $\mathrm{NH}_{3}$ storage in the catalyst.

An engine-dynamometer test setup with a full production aftertreatment system was developed and instrumented for experimental studies of the diesel aftertreatment system under various conditions. The SCR engine experimental data suitable for system characterization and model development were collected in the test cell. The test cell is 
capable of running both steady state and automated transient cycle tests. A surrogate HD-FTP cycle was developed to simulate the representative transients in order to study the aftertreatment system performance under typical transient conditions in the test cell. An exhaust heater was installed to independently control the temperature of the exhaust gas into the aftertreatment system.

Steady state SCR engine experiments were performed to collect the SCR data under different temperatures and varying upstream DEF injection conditions. A series of transient SCR experiments with different DEF injection levels were performed.

The Cu-zeolite SCR core taken from an identical catalyst was tested in a flow reactor at ORNL using test protocols designed to study the SCR performance under different space velocities, temperatures and inlet gaseous compositions. A Spaci-IR technique was utilized to measure the axially resolved gaseous concentrations in the SCR catalyst to quantify the axial concentration and $\mathrm{NH}_{3}$ storage profiles. The reactor data were used for calibration of the high fidelity SCR model to determine the storage capacities and the kinetic parameters associated with different SCR reactions included in the model. A calibration procedure and the identified model parameters were presented. The simulation results of the calibrated model were compared to the experimental results.

The high fidelity SCR model was calibrated to the steady state engine experimental data using the model parameters identified from the reactor data as a basis. A different set of the model parameters were determined for the SCR engine data. The heat transfer coefficients were identified from the temperature measurements during the transient cycle tests. The calibrated model was validated by simulating both the steady state and transient experiments and comparing the simulation results with the experimental measurements. The model and the kinetic parameters have been used by Surenahalli, et al to develop a reduced order SCR estimator model [22].

The $\mathrm{NH}_{3}$ measurement delay in the SCR engine experiments caused by the $\mathrm{NH}_{3}$ adsorption in the sampling line was experimentally studied. A sample line model which simulates the $\mathrm{NH}_{3}$ adsorption and desorption processes in the sample line was 
developed. The model consists of a $\mathrm{NH}_{3}$ storage site and includes $\mathrm{NH}_{3}$ adsorption and desorption reactions. The model parameters including the $\mathrm{NH}_{3}$ storage capacity in the sampling line, $\mathrm{NH}_{3}$ adsorption and desorption reaction rates were determined from sample line experimental data.

The $\mathrm{NH}_{3}$ maldistribution at the SCR inlet in the engine experiments were quantified through measuring the species concentrations at different radial locations of the SCR outlet face in the engine test cell. The effects of the $\mathrm{NH}_{3}$ maldistribution on the SCR performance and the kinetic model parameters were studied through simulations with a multi-channel SCR model consisting of 24 SCR channels running in parallel. The differences in the kinetic model parameters between the models calibrated to the reactor and engine data were explained by the $\mathrm{NH}_{3}$ maldistribution at the inlet to the SCR on the engine.

The hydrothermal aging effects were studied by comparing the Spaci-IR experimental results for both de-greened (at $700^{\circ} \mathrm{C}$ for 4 hours) and aged (at $800^{\circ} \mathrm{C}$ for 16 hours) SCR catalysts. The hydrothermal aging effects on the $\mathrm{NH}_{3}$ storage capacity and the SCR reaction kinetics were quantified.

\subsection{Conclusions}

The 2-site modeling approach is necessary for the Cu-zeolite SCR. Adding the $2^{\text {nd }}$ site which adsorbs and desorbs $\mathrm{NH}_{3}$ improved the prediction accuracy in the axially resolved $\mathrm{NH}_{3}$ storage. A $2^{\text {nd }}$ standard SCR reaction with higher $\mathrm{NH}_{3}$ to $\mathrm{NO}$ stoichiometric ratio is necessary for the model to simulate the $\mathrm{NH}_{3}$ overconsumption observed under the standard SCR reaction conditions in the reactor experiments. The high fidelity SCR model calibrated to the reactor data is able to capture the effects of space velocity and temperature on the reaction rates. The calibrated model is able to simulate the SCR outlet concentrations of $\mathrm{NO}, \mathrm{NO}_{2}, \mathrm{~N}_{2} \mathrm{O}$ and $\mathrm{NH}_{3}$ as well as the $\mathrm{NH}_{3}$ storage under space velocities from 60,000 to $120,000 \mathrm{hr}^{-1}$ and temperatures from 200 to $425^{\circ} \mathrm{C}$ with simulation cost values consistently below 30 . 
The high fidelity SCR model calibrated to the engine data accounts for the complex engine exhaust conditions and is able to simulate the SCR outlet concentrations of NO, $\mathrm{NO}_{2}, \mathrm{NH}_{3}$ and $\mathrm{NH}_{3}$ storage under steady state and transient conditions. A maximum temperature difference of $30^{\circ} \mathrm{C}$ was measured between the SCR inlet and outlet for the transient cycle. The difference was caused by the heat transfer between the gas flow, the substrate and the ambient. The heat transfer equations with heat transfer coefficients of 13.5 and $0.015 \mathrm{~W} / \mathrm{m}^{2} / \mathrm{K}$ are necessary for the SCR model to predict the outlet temperatures under transient conditions. Considering the axial temperature gradients in the model helps improve the model simulation accuracy.

The effects of the SCR inlet $\mathrm{NO}_{2} / \mathrm{NO}_{x}$ ratio on the $\mathrm{NO}_{\mathrm{x}}$ reduction efficiency and the $\mathrm{NH}_{3}$ slip were studied through simulation studies of the surrogate HD-FTP cycle. The simulation results showed that the SCR $\mathrm{NO}_{x}$ conversion efficiency is highest $(88.2 \%)$ with an inlet $\mathrm{NO}_{2} / \mathrm{NO}_{\mathrm{x}}$ ratio of 0.5 . The $\mathrm{NH}_{3}$ stored on the catalyst decreased with increase in inlet $\mathrm{NO}_{2} / \mathrm{NO}_{x}$ ratio under transient conditions. The $\mathrm{NH}_{3}$ slip is minimum for the inlet $\mathrm{NO}_{2} / \mathrm{NO}_{\mathrm{x}}$ ratio greater than 0.6.

The $\mathrm{NH}_{3}$ measurements from the emission analyzer were delayed by 3 to 6 minutes in the engine experiments. This phenomenon was determined to be caused by the $\mathrm{NH}_{3}$ adsorption and desorption process in the sample line. The effect of this delay needs to be considered when calibrating the model to the experimental data. The sample line model developed from experimental data is able to simulate the $\mathrm{NH}_{3}$ adsorption and desorption process in the sampling line. The $\mathrm{NH}_{3}$ storage capacity for the sample line heated to $190^{\circ} \mathrm{C}$ was determined to be $0.12 \mathrm{gmol} / \mathrm{m}^{3}$. The $\mathrm{NH}_{3}$ adsorption and desorption reaction rate constants were determined to be $3.5 \mathrm{~m}^{3} / \mathrm{gmol} \cdot \mathrm{s}$ and $0.011 / \mathrm{s}$ respectively. Incorporating the sampling model into the SCR model simulations reduced the simulation cost value by about $34 \%$ for the steady state time history $\mathrm{NH}_{3}$ slip predictions.

Differences in the model parameters were observed from the model calibrations developed separately from the reactor and engine experimental data. This indicates that the SCR performed differently in the engine exhaust conditions than the reactor test 
environments. The reactor experiments are important for characterization of the SCR performance and to study the basic kinetics of the catalyst. A SCR model calibrated to the engine data accounts for the complex engine environments and is suitable for onvehicle control and diagnostic function developments.

The $\mathrm{NH}_{3}$ maldistribution was determined to be present at the SCR inlet in the engine experiments and was quantified through experiments and data processing. The results showed that the uniformity index (UI) of the $\mathrm{NH}_{3}$ at the SCR inlet is less than 0.8 with the original hardware setup and is improved to about 0.9 with a swirl mixer installed into the SCR inlet cone. The results of the simulation studies showed that the $\mathrm{NH}_{3}$ maldistribution reduces the SCR $\mathrm{NO}_{x}$ reduction efficiency by 5 to $10 \%$ and increases the $\mathrm{NH}_{3}$ slip about 10 to $20 \mathrm{ppm}$. The simulation results from a multi-channel SCR model with nonuniform $\mathrm{NH}_{3}$ input confirmed that the $\mathrm{NH}_{3}$ maldistribution contributes to the differences in the model parameters identified from the reactor and engine data. The SCR model calibrated to the engine data accounts for the $\mathrm{NH}_{3}$ maldistribution phenomena and serves as a basis for developing the reduced order model and computationally efficient state estimators.

The hydrothermal aging condition of $800^{\circ} \mathrm{C}$ for 16 hours has effects on both the $\mathrm{NH}_{3}$ storage capability of the catalyst and the kinetic parameters of the major SCR reactions. The $\mathrm{NH}_{3}$ storage capacity was reduced from 138 to $94.6 \mathrm{gmol} / \mathrm{m}^{3}$ compared to a degreened catalyst (de-green condition: $700^{\circ} \mathrm{C}$ for 4 hours). The hydrothermal aging also resulted in different $\mathrm{NH}_{3}$ adsorption/desorption kinetics and reduced activation energy/pre-exponential factor for $\mathrm{NH}_{3}$ oxidation, standard and fast SCR reactions. Both the reduction in $\mathrm{NH}_{3}$ storage capacity and change in SCR kinetics contributed to the changes in the axial storage and concentration profiles observed from the experiments.

\subsection{Recommendations}

An emission analyzer with the capability of measuring species including $\mathrm{NO}, \mathrm{NO}_{2}, \mathrm{NH}_{3}$, $\mathrm{N}_{2} \mathrm{O}$ and $\mathrm{HNCO}$ without interference is important for SCR testing. $\mathrm{N}_{2} \mathrm{O}$ is produced in the 
SCR under high $\mathrm{NO}_{2} / \mathrm{NO}_{x}$ ratios and low temperatures and $\mathrm{HNCO}$ is one of the urea decomposition products that might be present in the exhaust. Measurements of these species are helpful for further understanding the urea decomposition process and the SCR performance. A FTIR that can measure multiple gaseous concentrations in the diesel exhaust is recommended for the SCR testing.

The SCR performance at low temperatures $\left(<250^{\circ} \mathrm{C}\right)$ should be given more attention in the engine experiments. In this study, the SCR inlet temperature was limited to above $250^{\circ} \mathrm{C}$ to prevent effects caused by the formation of solid urea crystallization. An injection system with higher injection pressure or other technologies improving the injection quality and better mixer designs might be helpful to reduce the urea crystallization at low temperatures.

It is recommended to collect the SCR reactor data in a wider temperature range which covers from the idle or low load to the CPF active regeneration conditions. Efforts should be made to calibrate the model to the wider temperature window to improve the calibration. The recommended temperature range is from 150 to $600^{\circ} \mathrm{C}$.

The urea decomposition process is complex and directly affects the amount of available $\mathrm{NH}_{3}$ at the SCR inlet. Understanding the urea decomposition process and correctly modeling the process would be helpful to determine the actual $\mathrm{NH}_{3}$ concentration at the inlet of the SCR. Efforts are recommended to be made in the future to test and model the urea decomposition reactions. It requires overcoming the difficulties of measuring the urea decomposition products without causing damage to the analyzers. The ammonium nitrate formation and decomposition reactions are recommended to be added into the high fidelity SCR model to simulate the inhibition effects under temperatures below $300^{\circ} \mathrm{C}$ and $\mathrm{NO}_{2} / \mathrm{NO}_{x}$ ratios higher than 0.5 . Supplemental reactor experiments are necessary for collecting SCR data suitable for calibration of the reactions.

An engine-aftertreatment system model for the ECM that controls the SCR inlet $\mathrm{NO}_{2} / \mathrm{NO}_{\mathrm{x}}$ ratio to around 0.5 is important in order to achieve the highest $\mathrm{NO}_{\mathrm{x}}$ conversion efficiency with minimum $\mathrm{NH}_{3}$ slip. 


\section{References}

1. DOE/EIA, Alternatives to Traditional Transportation Fuels 2009, 2011

2. U.S. Energy Information Administration, Annual Energy Outlook 2012 Early Release Overview, 2012

3. Yanowitz J, Mccormick RL, Graboski MS. In-Use Emissions from Heavy-Duty Diesel Vehicles. Environmental Science \& Technology 2000;34:729-740, 2000.

4. EPA, DOT, NHTSA. Greenhouse Gas Emissions Standards and Fuel Efficiency Standards for Medium- and Heavy-Duty Engines and Vehicles, 2011.

5. Johnson TV. Diesel Emission Control - Last 12 Months in Review. SAE Technical Paper 2000-01-2817, 2000.

6. Johnson TV. Diesel Emission Control: 2001 in Review. SAE Technical Paper 2002-01-0285, 2002.

7. Johnson TV. Diesel Emission Control in Review - The Last 12 Months. SAE Technical Paper 2003-01-0039, 2003.

8. Johnson TV. Diesel Emission Control in Review. SAE Technical Paper 2007-010233, 2007.

9. Timothy V. Johnson. Diesel Emission Control in Review. SAE Technical Paper 2008-01-0069, 2008.

10. Johnson TV. Diesel Emission Control in Review. SAE Technical Paper 2009-010121, 2009.

11. Johnson TV. Review of Diesel Emissions and Control. SAE Technical Paper 2010-01-0301, 2010.

12. Johnson TV. Diesel Emissions in Review. SAE Technical Paper 2011-01-0304, 2011.

13. Johnson TV. Vehicular Emissions in Review. SAE Technical Paper 2012-010368, 2012.

14. Emission Standards, Available from: www.dieselnet.com.

15. Kim CH, Qi G, Dahlberg K, Li W. Strontium-Doped Perovskites Rival Platinum Catalysts for Treating $\mathrm{NO}_{x}$ in Simulated Diesel Exhaust. Science 2010;327:1624-1627, 2010. 
16. Massimo Colombo, Isabella Nova, Enrico Tronconi. A comparative study of the NH3-SCR reactions over a Cu-zeolite and a Fe-zeolite catalyst. Catalysis Today 2010;151:223-230, 2010.

17. Fujii S, Asako T. Design Optimization of Non-Catalyzed DPF from Viewpoint of Back Pressure in Ash loading State. SAE Technical Paper 2011-01-2091, 2011.

18. Chatterjee D, Burkhardt T, Weibel M, Nova I, Grossale A, Troncon E. Numerical Simulation of Zeolite- and V-Based SCR Catalytic Converters. SAE 2007-011136, 2007.

19. Steven J. Schmieg, Jong-Hwan Lee. Evaluation of Supplier Catalyst Formulations for the Selective Catalytic Reduction of $\mathrm{NO}_{x}$ With Ammonia SAE Technical Paper 2005-01-3881, 2005.

20. Cloudt R, Willems F, Heijden Pvd. Cost and Fuel Efficient SCR-only Solution for Post-2010 HD Emission Standards. SAE Technical Paper 2009-01-0915, 2009.

21. Selective Catalytic Reduction, Available from: http://en.wikipedia.org/wiki/Selective_catalytic_reduction.

22. Surenahalli HS, Parker GG, Johnson JH. Extended Kalman Filter Estimator for $\mathrm{NH}_{3}$ Storage, $\mathrm{NO}, \mathrm{NO}_{2}$ and $\mathrm{NH}_{3}$ Estimation in a SCR. SAE Technical Paper 2013-01-1581, 2013.

23. Deland SB, Development and Parameter Identification of An Iron-Zeolite SCR Catalyst Model Using Reactor Data, Master's thesis. Houghton, MI: Michigan Technological University; 2010.

24. Khair M, Lemaire J, Fischer S. Achieving Heavy-Duty Diesel NO $\mathrm{N}_{\mathrm{x}} / \mathrm{PM}$ Levels Below the EPA 2002 Standards - An Integrated Solution. SAE Technical Paper 2000-01-0187, 2000.

25. Khair M, Lemaire J, Fischer S. Integration of Exhaust Gas Recirculation, Selective Catalytic Reduction, Diesel Particulate Filters, and Fuel-Borne Catalyst for $\mathrm{NO}_{\mathrm{x}} / \mathrm{PM}$ Reduction. SAE Technical Paper 2000-01-1933, 2000.

26. Zelenka P, Aufinger H, Reczek W, Cartellieri W. Cooled EGR - A Key Technology for Future Efficient HD Diesels. SAE Technical Paper 980190, 1998.

27. Baert RSG, Beckman DE, Veen A. Efficient EGR Technology for Future HD Diesel Engine Emission Targets. SAE Technical Paper 1999-01-0837, 1999. 
28. Charlton S, Dollmeyer T, Grana T. Meeting the US Heavy-Duty EPA 2010 Standards and Providing Increased Value for the Customer. SAE Technical Paper 2010-01-1934, 2010.

29. Hums E, Joisten M, Mtiller R, Sigling R, Spielmann H. Innovative Lines of SCR Catalysis" $\mathrm{NO}_{x}$ Reduction for Stationary Diesel Engine Exhaust Gas and Dioxin Abatement for Waste Incineration Facilities. Catalysis Today 1996;27(1996):2934, 1996.

30. Nakajima F, Hamada I. The State-of-the-art Technology of $\mathrm{NO}_{\mathrm{x}}$ Control. Catalysis Today 1996;29:109-115, 1996.

31. Amon B, Fischer S, Hofmann L, Zürbig J. The SINOx System for Trucks to Fulfill the Future Emission Regulations. Topics in Catalysis 2001;16/17:187-191, 2001.

32. Fritz $\mathrm{N}$, Mathes $\mathrm{W}$, Zuerbig J, Mueller R. On-Road Demonstration of $\mathrm{NO}_{\mathrm{x}}$ Emission Control for Diesel Trucks with $\mathrm{SINO}_{\mathrm{x}}$ Urea SCR System. SAE Technical Paper 1999-01-0111, 1999.

33. Brodrick CJ, Farsh-chi M, Dwyer HA, Sperling D, Gouse SW, Doelling W, Hoelzer J, Jackson M. Urea-SCR System Demonstration and Evaluation for Heavy-Duty Diesel Trucks. SAE Technical Paper 1999-01-3722, 1999.

34. Miller WR, Klein JT, Mueller R, Doelling W, Zuerbig J. The Development of UreaSCR Technology for US Heavy Duty Trucks. SAE Technical Paper 2000-010190, 2000.

35. Scarnegie B, Miller WR, Bernd Ballmert WD, Fischer S. Recent DPF/SCR Results Targeting US2007 and Euro 4/5 HD Emissions. SAE Technical Paper 2003-01-0774, 2003.

36. McGeehan JA, Yeh S, Couch M. On the Road to 2010 Emissions: Field Test Results and Analysis with DPF-SCR System and Ultra Low Sulfur Diesel Fuel. SAE 2005-01-3716 2005.

37. Cavataio G, Girard JW, Lambert CK. Cu/Zeolite SCR on High Porosity Filters: Laboratory and Engine Performance Evaluations. SAE Technical Paper 2009-010897, 2009.

38. Kro"cher O, Elsener M. Combination of $\mathrm{V}_{2} \mathrm{O}_{5} / \mathrm{WO}_{3}-\mathrm{TiO}_{2}, \mathrm{Fe}-\mathrm{ZSM} 5$, and $\mathrm{Cu}-\mathrm{ZSM} 5$ Catalysts for the Selective Catalytic Reduction of Nitric Oxide with Ammonia. Ind Eng Chem Res 2008;47 (22):8588-8593, 2008. 
39. Girard JW, Montreuil C, Kim J, Cavataio G, Lambert C. Technical Advantages of Vanadium SCR Systems for Diesel $\mathrm{NO}_{x}$ Control in Emerging Markets. SAE Technical Paper 2008-01-1029, 2008.

40. Ura JA, Girard J, Cavataio G, Montreuil C, Lambert C. Cold Start Performance and Enhanced Thermal Durability of Vanadium SCR Catalysts. SAE Technical Paper 2009-01-0625, 2009.

41. Chapman DM, Fu G, Augustine S, Crouse J, Zavalij L, Watson M, Perkins-Banks D. New Titania Materials with Improved Stability and Activity for Vanadia-Based Selective Catalytic Reduction of $\mathrm{NO}_{x}$. SAE Technical Paper 2010-01-1179, 2010.

42. Gekas I, Vressner A, Johansen $\mathrm{K} . \mathrm{NO}_{\mathrm{x}}$ Reduction Potential of V-SCR Catalyst in SCR/DOC/DPF Configuration Targeting Euro VI Limits from High Engine $\mathrm{NO}_{x}$ Levels. SAE Technical Paper 2009-01-0626, 2009.

43. Kamasamudram K, Currier NW, Chen X, Yezerets A. Overview of the practically important behaviors of zeolite-based urea-SCR catalysts, using compact experimental protocol. Catalysis Today 2010;151:212-222, 2010.

44. Fedeyko JM, Chen H-Y, Ballinger TH, Weigert EC, Chang H-L, Cox JP, Andersen PJ. Development of Thermally Durable Cu/SCR Catalysts. SAE Technical Paper 2009-01-0899, 2009.

45. Kamasamudram K, Currier N, Szailer T, Yezerets A. Why Cu- and Fe-Zeolite SCR Catalysts Behave Differently At Low Temperatures. SAE Technical Paper 2010-01-1182, 2010.

46. Cavataio G, Girard J, Patterson JE, Montreuil C, Cheng Y, Lambert CK. Laboratory Testing of Urea-SCR Formulations to Meet Tier 2 Bin 5 Emissions. SAE Technical Paper 2007-01-1575, 2007.

47. Girard J, Cavataio G, Snow R, Lambert C. Combined Fe-Cu SCR Systems with Optimized Ammonia to $\mathrm{NO}_{x}$ Ratio for Diesel $\mathrm{NO}_{x}$ Control. SAE Int J Fuels Lubr 2008;1:603-610, 2008.

48. Grossale A, Nova I, Tronconi E. Ammonia Blocking of the "Fast SCR" Reactivity over a Commercial Fe-zeolite Catalyst for Diesel Exhaust Aftertreatment. Journal of Catalysis 2009;265:141-147, 2009. 
49. Grossale A, Nova I, Tronconi E, Chatterjee D, Weibel M. The Chemistry of the $\mathrm{NO} / \mathrm{NO}_{2}-\mathrm{NH}_{3}$ "fast" SCR Reaction over Fe-ZSM5 Investigated by Transient Reaction Analysis. Journal of Catalysis 2008;256:312-322, 2008.

50. Fedeyko JM, Chen B, Chen H-Y. Mechanistic study of the low temperature activity of transition metal exchanged zeolite SCR catalysts. Catalysis Today 2010;151 231-236, 2010.

51. Grossale A, Nova I, Tronconi E. Study of a Fe-zeolite-based System as $\mathrm{NH}_{3}$ SCR Catalyst for Diesel Exhaust Aftertreatment. Catalysis Today 2008;136:18$27,2008$.

52. Metkar PS, Harold MP, Balakotaiah V. Selective catalytic reduction of $\mathrm{NO}_{\mathrm{x}}$ on combined Fe- and Cu-zeolite monolithiccatalysts: Sequential and dual layer configurations Applied Catalysis B: Environmental 2012;111-112:67-80, 2012.

53. Sjo"vall H, Blint RJ, Olsson L. Detailed Kinetic Modeling of $\mathrm{NH}_{3} \mathrm{SCR}$ over $\mathrm{Cu}-$ ZSM-5. Applied Catalysis B: Environmental 2009;92:138-153, 2009.

54. Djerad S, Crocoll M, Kureti S, Tifouti L, Weisweiler W. Effect of Oxygen Concentration on the $\mathrm{NO}_{\mathrm{x}}$ Reduction with Ammonia over $\mathrm{V}_{2} \mathrm{O}_{5}-\mathrm{WO}_{3} / \mathrm{TiO}_{2}$ Catalyst. Catalysis Today 2006;113:208-214, 2006.

55. Goo JH, Irfan MF, Kim SD, Hong SC. Effects of $\mathrm{NO}_{2}$ and $\mathrm{SO}_{2}$ on Selective Catalytic Reduction of Nitrogen Oxides by Ammonia. Chemosphere 2007;67:718-723, 2007.

56. Girard J, Snow R, Cabaralo G, Lambert C. The Influence of Ammonia to $\mathrm{NO}_{\mathrm{x}}$ Ratio on SCR Performance. SAE Technical Paper 2007-01-1581, 2007.

57. Nova I, Colombo M, Tronconi E, Schmeisse V, Weibel M. The $\mathrm{NH}_{3}$ Inhibition Effect in the Standard SCR Reaction over a Commercial Fe-zeolite Catalyst for Diesel Exhaust Aftertreatment: An Experimental and Modeling Study. SAE Technical Paper 2011-01-1319, 2011.

58. Uberoi M, Pereira CJ. External Mass Transfer Coefficients for Monolith Catalysts Ind Eng Chem Res 1996;35:113-116, 1996.

59. Grossale A, Nova I, Tronconi E, Chatterjee D, Weibel M. $\mathrm{NH}_{3}-\mathrm{NO} / \mathrm{NO}_{2} \mathrm{SCR}$ for Diesel Exhausts Aftertreatment: Reactivity, Mechanism and Kinetic Modelling of Commercial Fe- and Cu-Promoted Zeolite Catalysts. Top Catal 2009;52:1837$1841,2009$. 
60. Choi J-S, Partridge WP, Daw CS. Spatially Resolved In situ Measurements of Transient Species Breakthrough During Cyclic, Low-Temperature Regeneration of a Monolithic $\mathrm{Pt} / \mathrm{K}_{\mathrm{Al}} \mathrm{O}_{3} \mathrm{NO}_{\mathrm{x}}$ Storage-Reduction Catalyst. Applied Catalysis A: General 2005;293:24-40, 2005.

61. Shakir O, Yezerets A, Currier NW, Epling WS. Spatially Resolving Concentration and Temperature Gradients during the Oxidation of Propylene on $\mathrm{Pt} / \mathrm{Al}_{2} \mathrm{O}_{3}$. Applied Catalysis A: General 2009;365:301-308, 2009.

62. Hou X, Epling WS, Schmieg SJ, Li W. Cu-Zeolite SCR Catalyst Thermal Deactivation Studied with FTIR Spatial Resolution. SAE Technical Paper 201101-1138, 2011.

63. Luo J-Y, Hou X, Wijayakoon P, Schmieg SJ, Li W, Epling WS. Spatially resolving SCR reactions over a Fe/zeolite catalyst. Applied Catalysis B: Environmental 2011;102:110-119, 2011.

64. Henry C, Kamasamudram K, Currier N, Yezerets A. Axially Resolved Performance of Cu-Zeolite SCR Catalysts. SAE Technical Paper 2012-01-1084, 2012.

65. J. Arregle, V. Bermu'dez, J.R. Serrano, E. Fuentes. Procedure for engine transient cycle emissions testing in real time. Experimental Thermal and Fluid Science 2006;30:485-496, 2006.

66. Castagnola M, Caserta J, Chatterjee S, Chen H-Y, Conway R, Fedeyko J, Klink W, Markatou P, Shah S, Walker A. Engine Performance of $\mathrm{Cu}$ - and Fe-Based SCR Emission Control Systems for Heavy Duty Diesel Applications. SAE Technical Paper 2011-01-1329, 2011.

67. Cloudt R, Saenen J, Eijnden Evd, Rojer C. Virtual Exhaust Line for Model-based Diesel Aftertreatment Development. SAE Technical Paper 2010-01-0888, 2010.

68. El-Behery A, Rideout G, Iretskaya S, Meloche E, Tadrous T, Rosenblatt D, Brown K. Development and Field Performance Validation of a Retrofit SCR System for on-Road Heavy-Duty Application. SAE Technical Paper 2010-011186, 2010.

69. Walkowicz K, Na K, Robertson W, Sahay K, Bogdanoff M, Weaver C, Carlson R. On-Road and In-Laboratory Testing to Demonstrate Effects of ULSD, B20 and 
B99 on a Retrofit Urea-SCR Aftertreatment System. SAE Technical Paper 200901-2733, 2009.

70. Abu-Ramadan E, Saha K, Li X. Numerical Modeling of the Impingement Process of Urea-Water Solution Spray on the Heated Walls of SCR Systems. SAE Technical Paper 2012-01-1301, 2012.

71. Dong $\mathrm{H}$, Shuai S, Wang J. Effect of Urea Thermal Decomposition on Diesel $\mathrm{NO}_{\mathrm{x}^{-}}$ SCR Aftertreatment Systems. SAE Technical Paper 2008-01-1544, 2008.

72. Xu L, Watkins W, Snow R, Graham G, McCabe R, Lambert C, III ROC. Laboratory and Engine Study of Urea-Related Deposits in Diesel Urea-SCR After-Treatment Systems. SAE Technical Paper 2007-01-1582, 2007.

73. Sluder CS, Storey JME, Lewis SA, Lewis LA. Low Temperature Urea Decomposition and SCR Performance. SAE Technical Paper 2005-01-1858, 2005.

74. Howard L. Fang, Herbert F.M. DaCosta. Urea thermolysis and $\mathrm{NO}_{\mathrm{x}}$ reduction with and without SCR catalysts. Applied Catalysis B: Environmental 2003;46:17-34, 2003.

75. M. Kleemann, M. Elsener, M. Koebel, A. Wokaun. Hydrolysis of Isocyanic Acid on SCR Catalysts. Ind Eng Chem Res 2000;39:4120-4126, 2000.

76. Abu-Ramadan E, Saha K, Li X. Modeling of the Injection and Decomposition Processes of Urea-Water-Solution Spray in Automotive SCR Systems. SAE Technical Paper 2011-01-1317, 2011.

77. McKinley TL, Alleyne AG. A Urea Decomposition Modeling Framework for SCR Systems. SAE Technical Paper 2009-01-1269, 2009.

78. Schaber PM, Colson J, Higgins S, Thielen D, Anspach B, Brauer J. Thermal Decomposition (Pyrolysis) of Urea in an Open Reaction Vessel. Thermochimica Acta 2004;424 131-142, 2004.

79. Choi E-Y, Nam I-S, Kim YG. TPD Study of Mordenite-Type Zeolites for Selective Catalytic Reduction of NO by $\mathrm{NH}_{3}$. Journal of Catalysts 1996;161:597-604, 1996.

80. Kato A, Matsuda S, Kamo T, Nakajima F, Kuroda H, Narita T. Reaction between $\mathrm{NO}$, and $\mathrm{NH}_{3}$, on Iron Oxide-Titanium Oxide Catalyst. Phys Chem 1981;85:40994102, 1981. 
81. Hamada I, Kato Y, Imada N, Kikkawa H, Yamada A. A Unique Titania-Based SCR $\mathrm{NO}_{x}$ Catalyst for Diesel Exhaust Emission Control. SAE Technical Paper 2005-01-1859, 2005.

82. Narayanaswamy K, He Y. Modeling of Copper-Zeolite and Iron-Zeolite Selective Catalytic Reduction (SCR) Catalysts at Steady State and Transient Conditions. SAE Technical Paper 2008-01-0615, 2008.

83. Schär $\mathrm{CM}$, Onder $\mathrm{CH}$, Geering HP. Control of an SCR Catalytic Converter System for a Mobile Heavy - Duty Application. IEEE Transaction on Control System Technology 2006;14:641-653, 2006.

84. Chi JN, DaCosta HFM. Modeling and Control of a Urea-SCR Aftertreatment System. SAE Technical Paper 2005-01-0966, 2005.

85. Devarakonda M, Parker G, Johnson JH, Strots V, Santhanam S. Model-Based Estimation and Control System Development in a Urea-SCR Aftertreatment System. SAE Technical Paper 2008-01-1324, 2008.

86. Shost M, Noetzel J, Wu M-C, Sugiarto T, Bordewyk T, Fulks G, Fisher GB. Monitoring, Feedback and Control of Urea SCR Dosing Systems for $\mathrm{NO}_{\mathrm{x}}$ Reduction: Utilizing an Embedded Model and Ammonia Sensing. SAE Technical Paper 2008-01-1325, 2008.

87. Colombo M, Nova I, Tronconi E. A Comparative Study of the $\mathrm{NH}_{3}-\mathrm{SCR}$ Reactions over a Cu-zeolite and a Fe-zeolite Catalyst. Catalysis Today 2010;151:223-230, 2010.

88. Centi G, Perathoner S, Biglino D, Giamello E. Adsorption and Reactivity of NO on Copper on - Alumina Catalysts. Journal of Catalysis 1995;152:75-92, 1995.

89. $\mathrm{Xu} \mathrm{L}$, McCabe RW, Hammerle $\mathrm{RH}$. $\mathrm{NO}_{\mathrm{x}}$ Self-Inhibition in Selective Catalytic Reduction with Urea (Ammonia) over a Cu-Zeolite Catalyst in Diesel Exhaust. Applied Catalysis B: Environmental 2002;39:51-63, 2002.

90. Schrade F, Brammer M, Schaeffner J, Langeheinecke K, Kraemer L. PhysicoChemical Modeling of an Integrated SCR on DPF (SCR/DPF) System. SAE Int J Engines 2012;5:958-974, 2012.

91. Nova I, Ciardelli C, Tronconi E, Chatterjee D, Weibel M. Unifying Redox Kinetics for Standard and Fast $\mathrm{NH}_{3}-\mathrm{SCR}$ over a $\mathrm{V}_{2} \mathrm{O}_{5}-\mathrm{WO}_{3} / \mathrm{TiO}_{2}$ Catalyst. AIChE Journal 2009;55:1514-1529, 2009. 
92. Forzatti $\mathrm{P}$, Nova I, Tronconi E. Removal of $\mathrm{NO}_{\mathrm{x}}$ from Diesel Exhausts: The New “Enhanced $\mathrm{NH}_{3}$-SCR" Reaction. SAE Technical Paper 2010-01-1181, 2010.

93. Devarakonda MN, Dynamic Modeling, Simulation and Development of ModelBased Control Strategies in a Urea-SCR Aftertreatment System in Heavy Duty Diesel Engines, PhD Thesis. Houghton: Michigan Technological University; 2007.

94. Watling TC, Tutuianu M, Desai MR, Dai J, Markatou P, Johansson A. Development and Validation of a Cu-Zeolite SCR Catalyst Model. SAE Technical Paper 2011-01-1299, 2011.

95. Lietti L, Nova I, Tronconi E, Forzatti P. Transient kinetic study of the SCR-DeNO reaction. Catalysis Today 1998;45:85-92, 1998.

96. Chatterjee D, Burkhardt T, Weibel M, Tronconi E, Nova I, Ciardelli C. Numerical Simulation of $\mathrm{NO} / \mathrm{NO}_{2} / \mathrm{NH}_{3}$ Reactions on SCR-Catalytic Converters: Model Development and Applications. SAE Technical Paper 2006-01-0468, 2006.

97. Markatou P, Dai J, Johansson A, Wassim Klink MC, Watling TC, Tutuianu M. FeZeolite SCR Model Development, Validation and Application. SAE Technical Paper 2011-01-1304, 2011.

98. Schuler A, Votsmeier M, Malmberg S, Gieshoff J, Drochner A, Vogel H, Kiwic P. Dynamic Model for the Selective Catalytic Reduction of $\mathrm{NO}$ with $\mathrm{NH}_{3}$ on $\mathrm{Fe}$ Zeolite Catalysts. SAE Technical Paper 2008-01-1323, 2008.

99. S.Metkar P, Balakotaiah V, Harold MP. Experimental Study of Masstransfer Limitations in Fe- and Cu-Zeolite-Based $\mathrm{NH}_{3}$ - SCR Monolithic Catalysts. Chemical EngineeringScience 2011;66:5192-5203, 2011.

100. Balakotaiah V, West DH. Shape Normalization and Analysis of The Mass Transfer Controlled Regime in Catalytic Monoliths. Chemical Engineering Science 2002;57:1269-1286, 2002.

101. West $\mathrm{DH}$, Balakotaiah V, Jovanovic Z. Experimental and Theoretical Investigation of the Mass Transfer Controlled Regime in Catalytic Monoliths. Catalysis Today 2003;88:3-16, 2003.

102. Shamim R. Effect of Heat and Mass Transfer Coefficients on the Performance of Automotive Catalytic Converters. International Journal of Engine Research 2003;4:129-141, 2003. 
103. Ullah U, Waldram SP, Bennett CJ, Truex T. Monolithic Reactors: Mass Transfer Measurements under Reacting Conditions. Chemical Engineering Science 1992;47:2413-2418, 1992.

104. Kapas N, Shamim T, Laing P. Effect of Mass Transfer on the Performance of Selective Catalytic Reduction (SCR) Systems. Journal of Engineering for Gas Turbines and Power 2011;133:032801, 2011.

105. Santos A, Bahamonde A, Schmid M, Avila P, Garcl'a-Ochoa F. Mass Transfer Influences on the Design of Selective Catalytic Reduction (SCR) Monolithic Reactors. Chemical Engineering and Processing 1997;37:117-124, 1997.

106. Ruggeri MP, Nova I, Tronconi E. Experimental and Modeling Study of the Impact of Interphase and Intraphase Diffusional Limitations on the DeNO ${ }_{x}$ Efficiency of a V-Based Extruded Catalyst For $\mathrm{NH}_{3}-\mathrm{SCR}$ Of Diesel Exhausts. Chemical Engineering Journal 2012;207-208:57-65, 2012.

107. Chatterjee D, Burkhardt T, Rappe T, Güthenk A, Weibel M. Numerical Simulation of DOC+DPF+SCR Systems: DOC Influence on SCR Performance. SAE Technical Paper 2008-01-0867, 2008.

108. Surenahalli HS, Parker GG, Johnson JH, Devarakonda MN. A Kalman Filter Estimator for a Diesel Oxidation Catalyst during Active Regeneration of a CPF. 2012 American Control Conference 2012;4969 - 4974

109. Williams A, Ratcliff M, Pedersen D, McCormick R. Effect of Unburned Methyl Esters on the $\mathrm{NO}_{x}$ Conversion of Fe-Zeolite SCR Catalyst. SAE Technical Paper 2009-01-2777, 2009.

110. Devarakonda M, Tonkyn R, Herling D. Hydrocarbon Effect on a Fe-zeolite UreaSCR Catalyst: An Experimental and Modeling Study. SAE Technical Paper 201001-1171, 2010.

111. Montreuil C, Lambert C. The Effect of Hydrocarbons on the Selective Catalyzed Reduction of $\mathrm{NO}_{x}$ over Low and High Temperature Catalyst Formulations. SAE Technical Paper 2008-01-1030, 2008.

112. Devarakonda M, Tonkyn R, Tran D, Lee J, Herling D. Modeling Species Inhibition of NO Oxidation In Urea-SCR Catalysts for Diesel Engine $\mathrm{NO}_{x}$ Control. ICEF 2010-35054, 2010. 
113. Girard J, Snow R, Cavataio G, Lambert C. Influence of Hydrocarbon Storage on the Durability of SCR Catalysts. SAE Technical Paper 2008-01-0767, 2008.

114. Cavataio G, Jen H-W, Warner JR, Girard JW, Kim JY, Lambert CK. Enhanced Durability of a Cu/Zeolite Based SCR Catalyst. SAE Technical Paper 2008-011025, 2008.

115. Schmieg SJ, Oh SH, Kim CH, Brown DB, Lee JH, Peden CHF, Kim DH. Thermal Durability of $\mathrm{Cu}-\mathrm{CHA} \mathrm{NH} \mathrm{N}_{3}-\mathrm{SCR}$ Catalysts for Diesel $\mathrm{NO}_{x}$ Reduction. Catalysis Today 2012;184:252-261, 2012.

116. Johansson A, Wallin U, Karlsson M, Isaksson A, Bush P. Investigation on Uniformity Indices Used for Diesel Exhaust Aftertreatment Systems. SAE Technical Paper 2008-01-0613, 2008.

117. McKinley TL, Alleyne AG, Lee C-F. Mixture Non-Uniformity in SCR Systems: Modeling and Uniformity Index Requirements for Steady-State and Transient Operation. SAE Technical Paper 2010-01-0883, 2010.

118. Karlsson M, Wallin U, Fredholm S, Jansson J, Wahlström G-O, Schär CM, Onder $\mathrm{CH}$, Guzzell L. A Combined 3D/Lumped Modeling Approach to Ammonia SCR After-treatment Systems: Application to Mixer Designs. SAE Technical Paper 2006-01-0469, 2006.

119. Hua L, Zhao Y-g, Hu J, Tao T, Shuai S-J. Comparison Between Air-Assisted and Airless Urea Spray for Diesel SCR System by PDA and CFD. SAE Technical Paper 2012-01-1081, 2012.

120. Zhang X, Romzek M. 3-D Numerical Study of Flow Mixing in Front of SCR for Different Injection Systems. SAE Technical Paper 2007-01-1578, 2007.

121. Chen M, Williams S. Modelling and Optimization of SCR-Exhaust Aftertreatment Systems. SAE Technical Paper 2005-01-0969, 2005.

122. Koltsakis GC, Samaras ZC, Echtle H, Chatterjee D, Markou P, Haralampous OA. Flow Maldistribution Effects on DPF Performance. SAE Technical Paper 200901-1280, 2009.

123. Martin AP, Will NS, Bordet A, Cornet P, Gondoin C, Mouton X. Effect of Flow Distribution on Emissions Performance of Catalytic Converters. SAE Technical Paper 980936, 1998. 
124. Hutton CR, An Experimental Investigation Into The Passive Oxidation Of Particulate Matter In A Catalyzed Particulate Filter, Master's Thesis. Michigan Technological University; 2010.

125. Ciardelli C, Nova I, Tronconi E, Ascherfeld M, Fabinski W. Combined Use of a Mass-Spectrometer and a UV Analyzer in the Dynamic Study of NH3-SCR for Diesel Exhaust Aftertreatment. Topics in Catalysis 2007;42-43:161-164, 2007.

126. Chilumukuru KP, An Experimental Study of Particulate Thermal Oxidation in a Catalyzed Filter during Active Regeneration, Master's Thesis. Houghton: Michigan Technological University; 2008.

127. Emission Test Cycles: Heavy-Duty FTP Transient Cycle, Available from: http://dieselnet.com/standards/cycles/ftp_trans.php.

128. Song X, Parker G, Johnson J, Naber J, Pihl J. A Modeling Study of SCR Reaction Kinetics from Reactor Experiments. SAE Technical Paper 2013-011576, 2013.

129. Pant A, Schmieg SJ. Kinetic Model of $\mathrm{NO}_{x}$ SCR Using Urea on Commercial $\mathrm{Cu}-$ Zeolite Catalyst. Ind Eng Chem Res 2011;50:5490-5498, 2011.

130. Metkar PS, P.Harold M, Balakotaiah V. Experimental and Kinetic Modeling Study of $\mathrm{NH}_{3}-\mathrm{SCR}$ of $\mathrm{NO}_{x}$ on Fe-ZSM-5, Cu-chabazite and Combined Fe-and Cuzeolite Monolithic Catalysts. Chemical Engineering Science 2013;87:51-66, 2013.

131. Joo K, Jo J-H, Kim C, Lee J-h, Kim H-j. The Study of $\mathrm{NO}_{\mathrm{x}}$ Reduction using UreaSCR System with CPF and DOC for Light Duty Vehicle; the Diesel $\mathrm{NO}_{\mathrm{x}}$ Reduction System. SAE Technical Paper 2008-01-1183, 2008.

132. Shiel K, Naber J, Johnson J, Hutton C. Catalyzed Particulate Filter Passive Oxidation Study with ULSD and Biodiesel Blended Fuel. SAE Technical Paper 2012-01-0837, 2012.

133. Song X, Parker G, Pihl J, Aging Impact on Ammonia Storage Report, Deliverable Report to DOE: DE-EE0000204-10,2013

134. Novaa I, Ciardellia C, Tronconia E, Chatterjeeb D, Weibelb M. $\mathrm{NH}_{3}-\mathrm{NO} / \mathrm{NO}_{2} \mathrm{SCR}$ for Diesel Exhausts After Treatment: Mechanism and Modelling of a Catalytic Converter. Topics in Catalysis 2007;42-43:43-46, 2007. 


\section{Appendix A. IMR-MS Start up and Calibration procedures}

The analyzer needs to be turned on at least two days before the test to allow enough time for the analyzer to be warmed up when testing. The sensitivity of a cold analyzer is not stable and the measurements shift a lot. The source gas bottles in the analyzer should be turned on to flush the gas lines and remove any possible oxygen leaked into the system. The oxygen in the system may cause defects of the filament which generates electrons. The analyzer needs to be connected to the computer via a crossover cable for the initial startup. Switch on the power switch to turn on the analyzer. The display on the RF generator shows the status of the analyzer. As the analyzer warms up it will have a red LED bar on the display. It will go to Red-Green after the analyzer is ready for operation. Then turn on the computer which is connected to the analyzer and has V\&F Viewer software installed. Open the V\&F View and connect to the analyzer by selecting "Connect" from the menu item "File $\rightarrow$ Connect". Then choose the right measurement method that was set up for the test from the upper part of the software interface. The procedures of setting up the measurement methods are available in the user manuals. Leave the analyzer under "Standby" or "Measure" condition to warm up.

The IMR-MS needs to be calibrated to the calibration gases before each test. The V\&F Viewer software provides two ways of calibration including an automatic calibration of the interested species selected and manual calibration of each species. The procedure of the automatic calibration can be found in the user manual. To start a manual calibration, select the calibration channel which is associated with the interested calibration gas from the "Sample inlet" function on the upper part of the software interface. This enables the analyzer to start measuring the calibration gas. Wait until the signal gets stabilized. Then right click the interested species from the "Molecule List" on the left hand side of the software interface and select "Channel Calibration". Type in the calibration gas concentration and click OK. Then repeat the procedure for each species 
that needs to be calibrated. The manual calibration should be performed after obtaining a good background by zeroing the analyzer with $\mathrm{N}_{2}$. A automatic calibration combined with manual calibration was usually performed to achieve a good calibration.

To shut down the analyzer, turn off the software first before switching off the power supply to prevent from losing data by switching off the power while running. Select "Shut down analyzer" from the tools menu of the V\&F Viewer program to turn off the software. Then switch off the power switch located at the front panel of the analyzer. Then turn off the source gas bottle to prevent any possible leakage when not running.

\section{Appendix B. Calibration of the DEF Injector}

The $\mathrm{NH}_{3}$ concentration at the SCR inlet generated from the DEF injection is determined by the DEF injection rate, urea properties, and exhaust flow rate. In order to achieve the desired $\mathrm{NH}_{3}$ concentration, it is important to accurately control the DEF injection flow rate injected into the exhaust flow. The DEF injection rate is controlled by sending flow rate command to the engine ECM through the Cummins proprietary software which inter communicates with the engine ECM. The injector is calibrated to accurately give the desired DEF flow rate. A calibration process conducted without running the engine is described as follows:

1. Disconnect the injector from the mount on the exhaust pipe.

2. Position a $500 \mathrm{ml}$ graduated cylinder underneath the DEF injector.

3. Start the DEF injection with a flow rate command of $0.02 \mathrm{ml} / \mathrm{sec}$. Continue the injection until measurable volume of DEF is collected in the cylinder.

4. Remove the graduated cylinder and place it on a level surface. Wait until there are no bubbles in the DEF collected, and then record the volume of DEF that is injected 
into the cylinder. Pour the DEF back to the tank, and start over at the next higher injection flow rate.

The relationship between the flow rate commands (Flowrate_Cmd) sent to the ECM and the actual DEF injection rates calculated (Flowrate_Act) is shown in Figure B.1. A linear trend line was added to characterize the relationship. The calibration process was repeated during the engine testing period to verify the repeatability of the injector. Two additional calibration curves are also given in Figure B.1.

The urea concentration of the DEF is $32.5 \%$ by weight. The decomposition of 1 urea molecule forms two $\mathrm{NH}_{3}$ molecules through a series of reactions[76]. As a result, the calculation of the available SCR inlet $\mathrm{NH}_{3}$ concentration when applying an injection command value of Flowrate_Cmd is given as the following equation.

$$
C_{N H_{3}}=\frac{\text { Flowrate }_{A c t} \times \rho_{D E F} \times 0.325 \times \frac{2}{M W_{\text {Urea }}}}{\frac{\text { Flowrate }_{E x h}}{M W_{E x h}}}=\frac{\left(\text { Flowrate }_{\text {Cmd }} \times 1.0177+0.0013\right) \times \rho_{\text {DEF }} \times 0.325 \times \frac{2}{M W_{\text {Urea }}}}{\frac{\text { Flowrate }_{\text {Exh }}}{M W_{\text {Air }}}}
$$

Where, $\rho_{\mathrm{DEF}}$ is the density of the DEF which is $1080 \mathrm{~kg} / \mathrm{m}^{3}$ under room condition. $\mathrm{MW}_{\text {urea }}$ is the molecular weight of the urea molecule which is $60 \mathrm{~g} / \mathrm{gmol}, \mathrm{MW}_{\mathrm{Exh}}$ is the molecular weight of the exhaust gas and it is considered to be equal to that of air which is 28.8 g/gmol. 


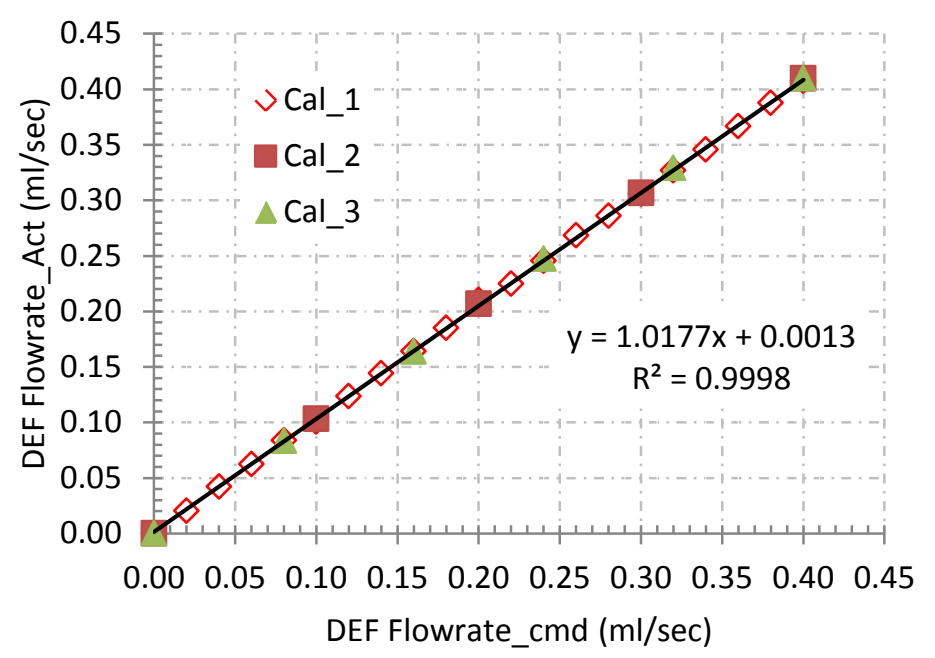

Figure B.1: DEF injector calibration curve

\section{Appendix C. Calculation of SCR Actual Space Velocity}

The calculations of the SCR actual and standard space velocities are described in this section. The SCR actual space velocity is the SCR space velocity under operating pressure and temperature conditions while the standard space velocity is calculated under a specific reference pressure and temperature. The space velocity was a ratio of the exhaust volumetric flow rate to the open catalyst volume as given in Equation C.1.The actual exhaust volumetric flow rate was the exhaust mass flow rate divided by the actual density of exhaust as shown in Equation C.2. In this calculation the actual exhaust density was assumed to be equal to the actual density of the air. The calculation of the actual air density is given in Equation C.3. Where, $\mathrm{P}$ is the absolute pressure in $\mathrm{Pa}, \mathrm{R}$ is the specific gas constant for dry air and equal to $287 \mathrm{~J} /(\mathrm{kg} \cdot \mathrm{K})$. T is the SCR temperature in $\mathrm{K}$. The standard exhaust volumetric flow rate was the exhaust mass flow rate divided by the standard density of exhaust as shown in Equation C.4. The standard density of the exhaust is assumed to be equal to the density of air under $1 \mathrm{~atm}$ and $0^{\circ} \mathrm{C}$. The catalyst volume used in Equation C.1 is the open volume of the catalyst which is the 
open area times the catalyst length. The open volume was calculated by multiplying the area of each catalyst cell and the number of inlet cells. The open catalyst volume calculation is given in Equation C.5.

$$
\text { SV }\left(h r^{-1}\right)=\frac{\text { Exh Volumetric Flow Rate }\left(\mathrm{m}^{3} / \mathrm{hr}\right)}{\text { Open Catalyst Volume }\left(\mathrm{m}^{3}\right)}
$$

Actual Exh Volumetric Flow Rate $\left(\mathrm{m}^{3} / \mathrm{hr}\right)=\frac{\text { Exh Mass Flow Rate }(\mathrm{kg} / \mathrm{hr})}{\text { Actual Exh Density }\left(\mathrm{kg} / \mathrm{m}^{3}\right)}$

$$
\text { Actual Air Density }\left(\mathrm{kg} / \mathrm{m}^{3}\right)=\frac{P(P a)}{R(J / \mathrm{kg} \cdot K) T(K)}
$$

Standard Exh Volumetric Flow Rate $\left(\mathrm{m}^{3} / \mathrm{hr}\right)=\frac{\text { Exh Mass Flow Rate }(\mathrm{kg} / \mathrm{hr})}{\text { Standard Exh Density }\left(\mathrm{kg} / \mathrm{m}^{3}\right)}$

Open Catalyst Volume $\left(\mathrm{m}^{3}\right)=\#$ of Cells $\times$ Cell Width ${ }^{2}\left(\mathrm{~m}^{2}\right) \times$ Catalyst Length $(\mathrm{m})$

\section{Appendix D. Fuel Mass Flow Rate Calculation for Transient Test}

The exhaust mass flow rate is an important parameter to determine the SCR space velocity and is an input for the SCR model. The exhaust mass flow rate is the sum of the intake mass flow rate calculated from the pressure drop across the LFE and the measured fuel mass flow rate. Due to limitations of the AVL fuel system, real time fuel mass flow rate of the engine during transient cycles cannot be accurately measured. In order to determine the engine fuel mass flow rate, a calculation based on the exhaust measurements was performed. 
In this calculation, it was assumed that the lean combustion products included only $\mathrm{H}_{2} \mathrm{O}$, and $\mathrm{CO}_{2}$ with excess $\mathrm{O}_{2}$ and $\mathrm{N}_{2}$. Other products including $\mathrm{HC}, \mathrm{NO}_{x}$, and $\mathrm{PM}$ were ignored because of their negligible levels compared to the other species to reduce calculation complexity. The diesel fuel composition was assumed to be $\mathrm{C}_{12} \mathrm{H}_{24}$. The combustion process is given in Equation D.1.

$$
2 \mathrm{C}_{12} \mathrm{H}_{23}+\mathrm{a}\left(\mathrm{O}_{2}+3.76 \mathrm{~N}_{2}\right)=24 \mathrm{CO}_{2}+23 \mathrm{H}_{2} \mathrm{O}+\mathrm{y} \mathrm{O}_{2}+3.76 \mathrm{a} \mathrm{N}_{2}
$$

Equation D.2 can be derived from the $\mathrm{O}_{2}$ balance between the two sides of the reaction.

$$
2 a=24 \times 2+23+2 y
$$

The model fraction of $\mathrm{O}_{2}$ in the products time 100 is the wet $\mathrm{O}_{2}$ concentration in percentage measured by the IMR-MS. This relationship is given in Equation D.3.

$$
\left[O_{2}\right] w=100 y /(24+23+y+3.76 a)
$$

The coefficient a for the air in reactant side and the coefficient $y$ for the $\mathrm{O} 2$ in product side of Equation D.1 can be solved from Equation D.2 and D.3. The air fuel ratio was calculated based on the reactants on the left hand side of Equation D.1. The calculation is given in Equation D.4. The fuel mass flow rate can be determined by multiplying the calculated air fuel ratio with the measurement intake air mass flow rate.

$$
A / F=a(16 \times 2+3.76 \times 14 \times 2) /[2 \times(12 \times 12+23 \times 1)]
$$

\section{Appendix E. Calibration of the $\mathbf{N H}_{3}$ Sensor}

Experimental results show that the $\mathrm{NH}_{3}$ slip measurements from the $\mathrm{NH}_{3}$ sensor and the IMR-MS are off by about $10 \%$ because they have different sensitivity to $\mathrm{NH}_{3}$. In order to compare the $\mathrm{NH}_{3}$ measurements from the $\mathrm{NH}_{3}$ sensor and the IMR-MS, it is necessary to calibrate the $\mathrm{NH}_{3}$ sensor to the IMR-MS so that they give equal measurements when 
measuring the same gas components. The IMR-MS is calibrated before each test using the calibration gas with certified concentration of $103.8 \mathrm{ppm}$ balancing with $\mathrm{N}_{2}$.

For calibration of the $\mathrm{NH}_{3}$ sensor, SCR engine tests was performed with different upstream DEF injection rates to form different levels of $\mathrm{NH}_{3}$ slip which is measured by both the $\mathrm{NH}_{3}$ sensor and the IMR-MS at the same time. For each $\mathrm{NH}_{3}$ slip level, the gas sample was taken continuously until the $\mathrm{NH}_{3}$ measurements from both instruments reached steady state for 3 minutes. Another DEF injection rate was then carried out while continuing to measure $\mathrm{NH}_{3}$ downstream of the SCR. The test setup has been introduced in the previous sections. The test was performed during the CPF regeneration process with a SCR inlet temperature of $510^{\circ} \mathrm{C}$ so that the SCR reached the equilibrium state more quickly because the $\mathrm{NH}_{3}$ storage capacity of the SCR catalyst is significantly lower at higher temperatures. Then the steady state $\mathrm{NH}_{3}$ measurements from both instruments were compared and the ratio between the two measurements was calculated for each $\mathrm{NH}_{3}$ level. The average of each calculated ratio was considered to be the factor used to scale the $\mathrm{NH}_{3}$ sensor measurement to the IMR-MS measurement. The measured concentrations and the calculated ratios are given in Table E.1. The average of the calculated ratios was used as the $\mathrm{NH}_{3}$ sensor calibration factor.

Table E.1: $\mathrm{NH}_{3}$ sensor calibration results

\begin{tabular}{|c|c|c|c|c|c|c|c|}
\hline Speed & Load & $\begin{array}{c}\text { Exh } \\
\text { mass } \\
\text { flowrate }\end{array}$ & $\begin{array}{c}\text { SCR } \\
\text { Inlet } \\
\text { Temp }\end{array}$ & ANR & $\begin{array}{c}\mathbf{N H}_{3} \\
\text { Sensor }\end{array}$ & $\begin{array}{c}\mathbf{N H}_{3} \\
\mathbf{M S}\end{array}$ & $\begin{array}{c}\text { Ratio of } \\
\mathbf{N H}_{3} \\
\text { Conc. }\end{array}$ \\
\hline $\mathbf{r p m}$ & $\mathbf{N} \cdot \mathbf{m}$ & $\mathbf{k g} / \mathbf{m i n}$ & ${ }^{\circ} \mathbf{C}$ & $\mathbf{n o n e}$ & $\mathbf{p p m}$ & $\mathbf{p p m}$ & none \\
\hline 2310 & 281 & 11.17 & 514 & 0.00 & 0 & 0 & - \\
\hline 2310 & 279 & 11.17 & 513 & 1.40 & 24 & 21 & 1.15 \\
\hline 2310 & 276 & 11.18 & 514 & 1.60 & 46 & 38 & 1.21 \\
\hline 2310 & 284 & 11.19 & 513 & 1.80 & 74 & 63 & 1.18 \\
\hline 2310 & 276 & 11.18 & 514 & 2.00 & 94 & 81 & 1.17 \\
\hline 2310 & 288 & 11.19 & 514 & 2.19 & 126 & 108 & 1.16 \\
\hline 2310 & 285 & 11.20 & 517 & 0.00 & 0 & 5 & - \\
\hline
\end{tabular}




\section{Appendix F. Processing of the Spaci-IR}

\section{Data}

The gaseous concentrations and the $\mathrm{NH}_{3}$ storage at each axial measurement position under the equilibrium state of each test protocol step are important for the purpose of characterizing the SCR internal state and calibration of the SCR model. The gaseous concentrations can be calculated by averaging the measurement data recorded after the SCR reached equilibrium states. The $\mathrm{NH}_{3}$ storage under different SCR conditions was calculated by integrating the $\mathrm{NH}_{3}$ adsorbed on the catalyst or the $\mathrm{NO}_{x}$ reduced by the stored $\mathrm{NH}_{3}$ and $\mathrm{NH}_{3}$ slip after the $\mathrm{NH}_{3}$ was turned off. The calculation procedures for the measurement locations of 0.6 of the catalyst length and the temperature of $250^{\circ} \mathrm{C}$ are introduced below. The gaseous concentrations of $\mathrm{NO}, \mathrm{NO}_{2}$ and $\mathrm{NH}_{3}$ under this condition are shown in Figure F.1

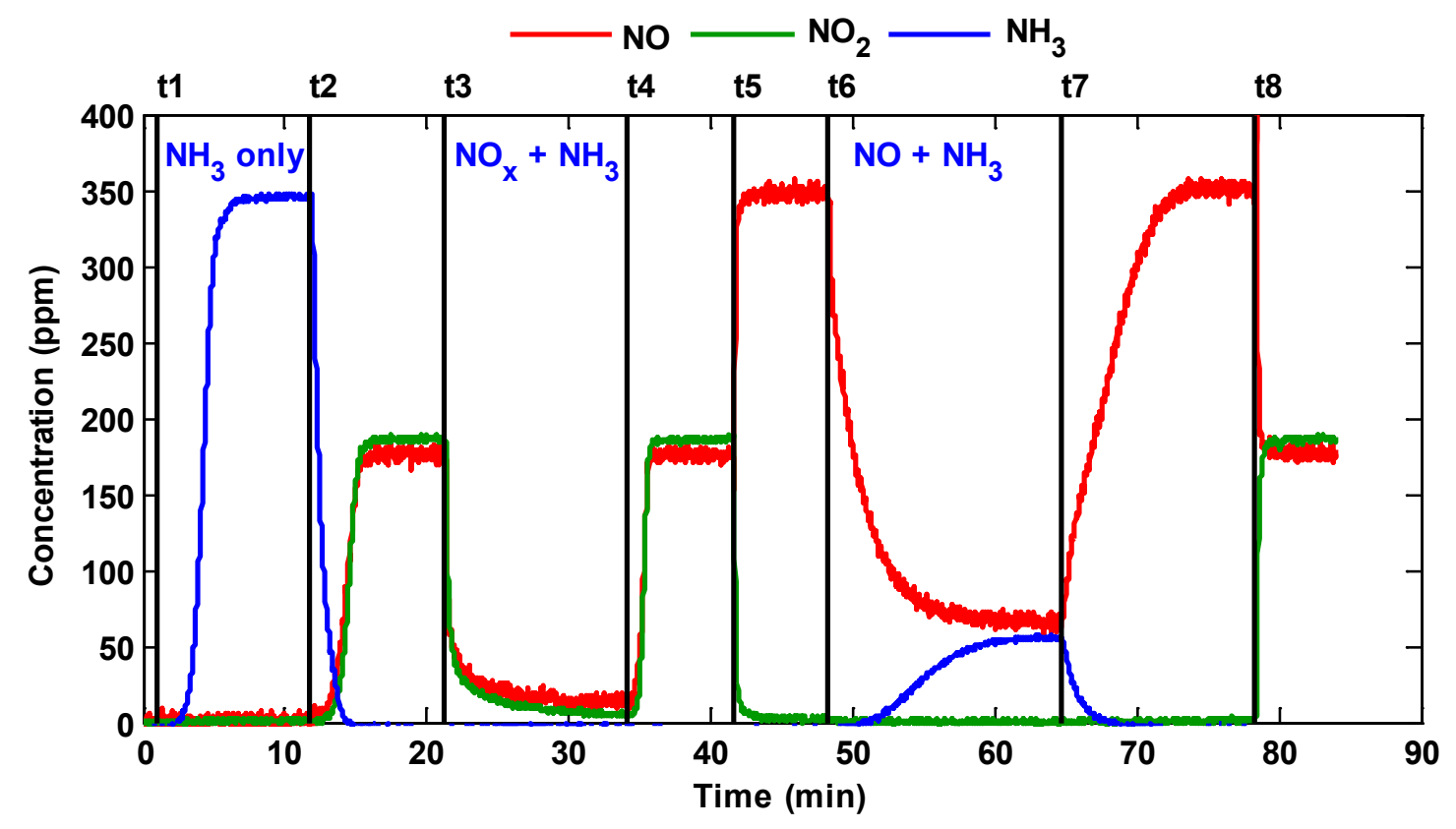

Figure F.1: Gaseous concentrations of $\mathrm{NO}, \mathrm{NO}_{2}$ and $\mathrm{NH}_{3}$ at the 0.6 of the catalyst length under temperature of $250^{\circ} \mathrm{C}$ 
The vertical black lines in Figure F.1 are the critical change points when the inlet gas composition was changed. The time of the change points were represented by $\mathrm{t} 1$-t 8 . As marked in the figure, the gaseous concentrations and $\mathrm{NH}_{3}$ storage under the equilibrium states of $\mathrm{NH}_{3}$ only, $\mathrm{NO}_{\mathrm{x}}+\mathrm{NH}_{3}, \mathrm{NO}+\mathrm{NH}_{3}$ sections are important. The gaseous concentrations at the time points $t 2, t 4$, and $t 7$ were considered as the equilibrium concentrations.

The $\mathrm{NH}_{3}$ storage under the equilibrium of the $\mathrm{NH}_{3}$ only section was calculated by integrating the difference between inlet and measured outlet $\mathrm{NH}_{3}$ concentrations over the time period of this section. The calculation is given in Equation F.1. The $\mathrm{NH}_{3}$ storage values under during the $\mathrm{NO}_{x}+\mathrm{NH}_{3}$ and $\mathrm{NO}+\mathrm{NH}_{3}$ sections were calculated by integrating the $\mathrm{NO}_{x}$ reduced by the stored $\mathrm{NH}_{3}$ and $\mathrm{NH}_{3}$ slip after the inlet $\mathrm{NH}_{3}$ was turned off until the $\mathrm{NH}_{3}$ adsorbed on the catalyst was cleaned up. The calculation is given in Equation F.2

$$
\begin{gathered}
N H_{3 s t o}=M \cdot \int_{t 1}^{t 2}\left(N H_{3_{-} \text {in }}-N H_{3_{-} \text {out }}(t)\right) d t \\
N H_{3 s t o}=M \cdot \int_{t(i)}^{t(i+1)}\left(N O_{x_{-} \text {in }}-N O_{x_{-} \text {out }}(t)+N H_{3_{\text {_out }} \text { out }}(t)\right) d t, \quad(i=4,7)
\end{gathered}
$$

Where, $\mathrm{M}$ is molar flow rate in $\mathrm{gmol} / \mathrm{min}$ calculated based on the space velocity and the catalyst dimensions as shown in Equation F.3. $V_{\text {cat }}$ is the catalyst volume calculated based on the diameter and length of the core sample. $P_{\text {ref }}$ and $T_{\text {ref }}$ are the reference pressure and temperature which were $1 \mathrm{~atm}$ and $0^{\circ} \mathrm{C}$ respectively. $\mathrm{R}$ is the global ideal gas constant. The space velocity of $70,000 \mathrm{hr}^{-1}$ was used in the storage calculations.

$$
M=\frac{\dot{m}_{e x h}}{M W_{e x h}}=\frac{S V \cdot V_{c a t}}{60} \cdot \frac{P_{r e f}}{R T_{r e f}}
$$

The storage calculations were also performed for the axial measurement position before the catalyst, which was the inlet measurement. This calculated storage accounted for the $\mathrm{NH}_{3}$ storage resulted from gas transportation in the sampling system of the analyzer and was subtracted from the $\mathrm{SCR}$ internal $\mathrm{NH}_{3}$ storage values. 
The gaseous concentrations and the $\mathrm{NH}_{3}$ storage calculations were conducted for different axial measurement positions and temperatures. The equilibrium state $\mathrm{NO}_{\mathrm{x}}$ and $\mathrm{NH}_{3}$ concentrations as a function of axial position at $\mathrm{NO}_{\mathbf{x}}+\mathrm{NH}_{3}$ and $\mathrm{NO}+\mathrm{NH}_{3}$ sections for three temperatures are shown in Figures F.2 and F.3. The equilibriums state $\mathrm{NH}_{3}$ storage at different sections as a function of the axial position for different temperatures are shown in Figures F.4, F.5, and Figure F.6.

Under the $\mathrm{NH}_{3}$ only conditions, the $\mathrm{NH}_{3}$ storage increased linearly along with the axial position, meaning that the $\mathrm{NH}_{3}$ was uniformly stored on the catalyst along the catalyst length. Under the $\mathrm{NO}_{\mathrm{x}}+\mathrm{NH}_{3}$ conditions, the $\mathrm{NH}_{3}$ storage was increasing at the first half of the catalyst and reached constant after the position of 0.6. The $\mathrm{NO}_{x}$ reduction reactions mainly took place on the first half of the catalyst and the $\mathrm{NH}_{3}$ was completely consumed at the position of 0.6 , causing no $\mathrm{NH}_{3}$ stored after that position. Under the $\mathrm{NO}$ $+\mathrm{NH}_{3}$ conditions, the SCR reaction took place along the catalyst length and higher $\mathrm{NH}_{3}$ storage was observed than the $\mathrm{NO}_{\mathbf{x}}+\mathrm{NH}_{3}$ conditions. The $\mathrm{NH}_{3}$ storage decreased as increase in SCR temperature, because higher temperature resulted in greater reaction rates that consuming more stored $\mathrm{NH}_{3}$. The temperature effects on the reaction rates of $\mathrm{NO}+\mathrm{NH}_{3}$ SCR reactions were more obvious than the $\mathrm{NO}_{x}+\mathrm{NH}_{3} \mathrm{SCR}$ relations, causing greater differences in axially resolved $\mathrm{NO}_{x}$ concentrations between different temperatures. 


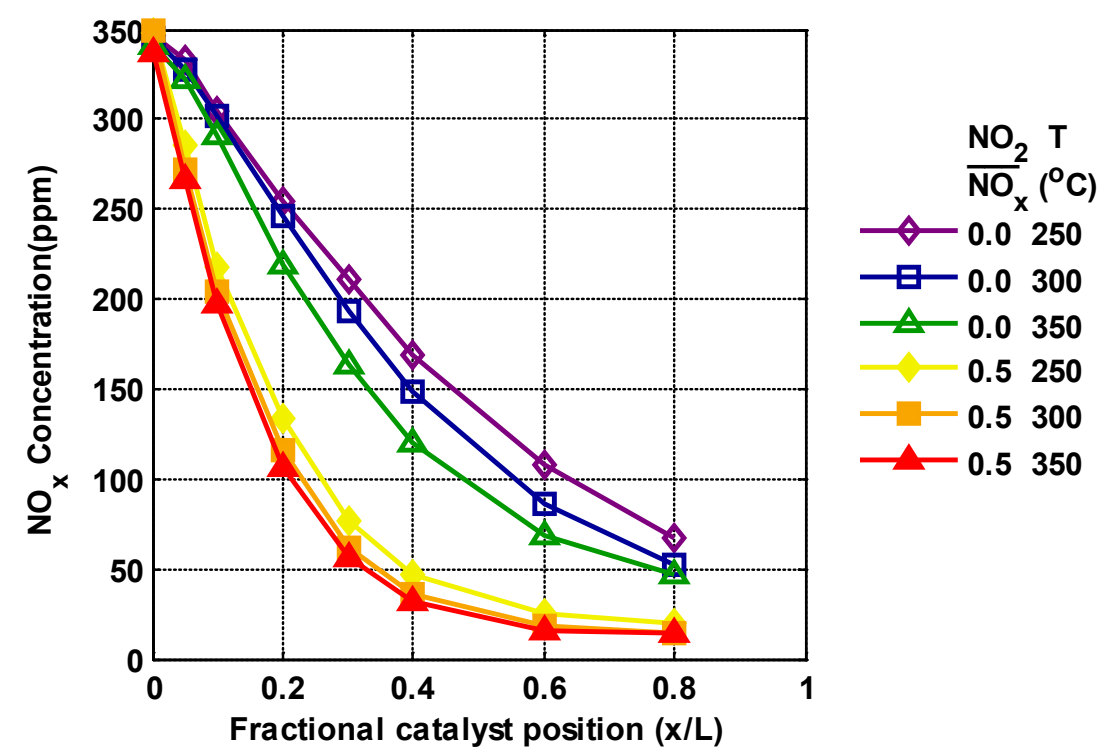

Figure F.2: $\mathrm{NO}_{x}$ concentrations at equilibrium state of $\mathrm{NO}_{x}+\mathrm{NH}_{3}$ and $\mathrm{NO}+\mathrm{NH}_{3}$ sections as a function of axial position for three temperatures

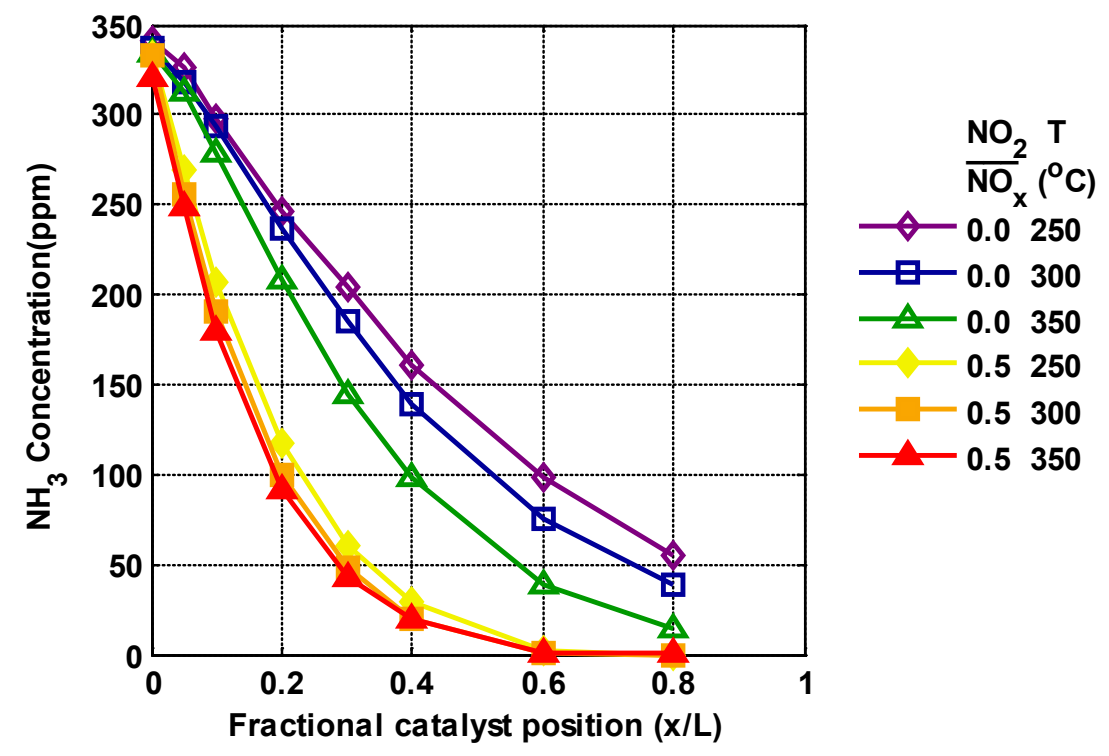

Figure F.3: $\mathrm{NO}_{x}$ concentrations at equilibrium state of $\mathrm{NO}_{x}+\mathrm{NH} 3$ and $\mathrm{NO}+\mathrm{NH}_{3}$ sections as a function of axial position for three temperatures 


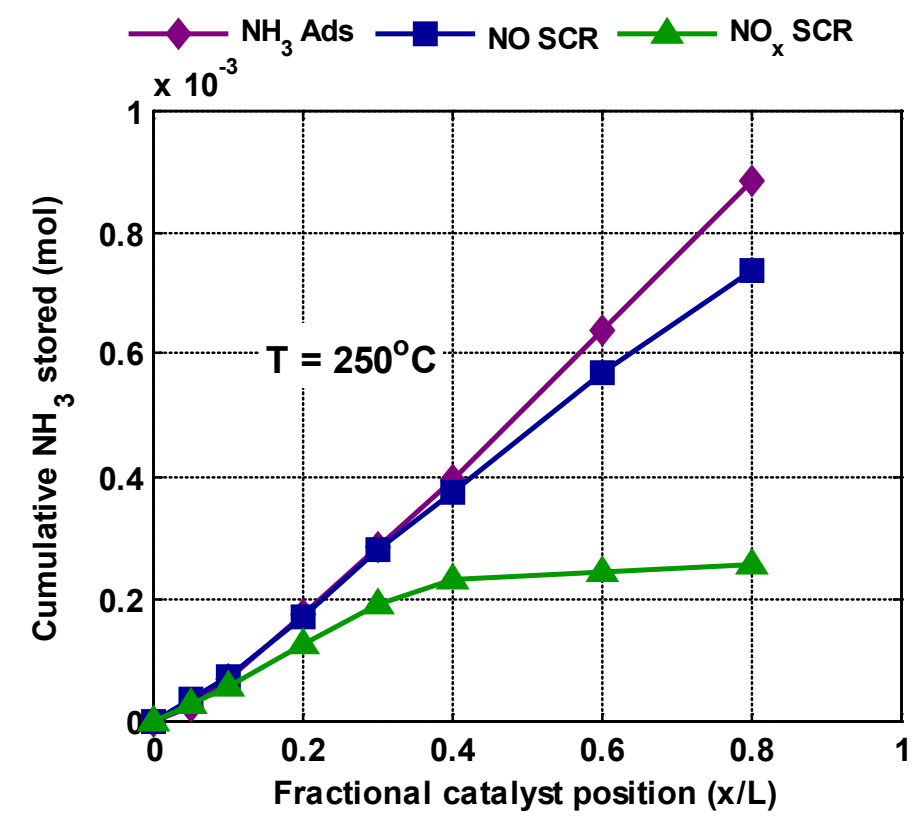

Figure F.4: $\mathrm{NH}_{3}$ storage as a function of axial position for the temperature of $250^{\circ} \mathrm{C}$

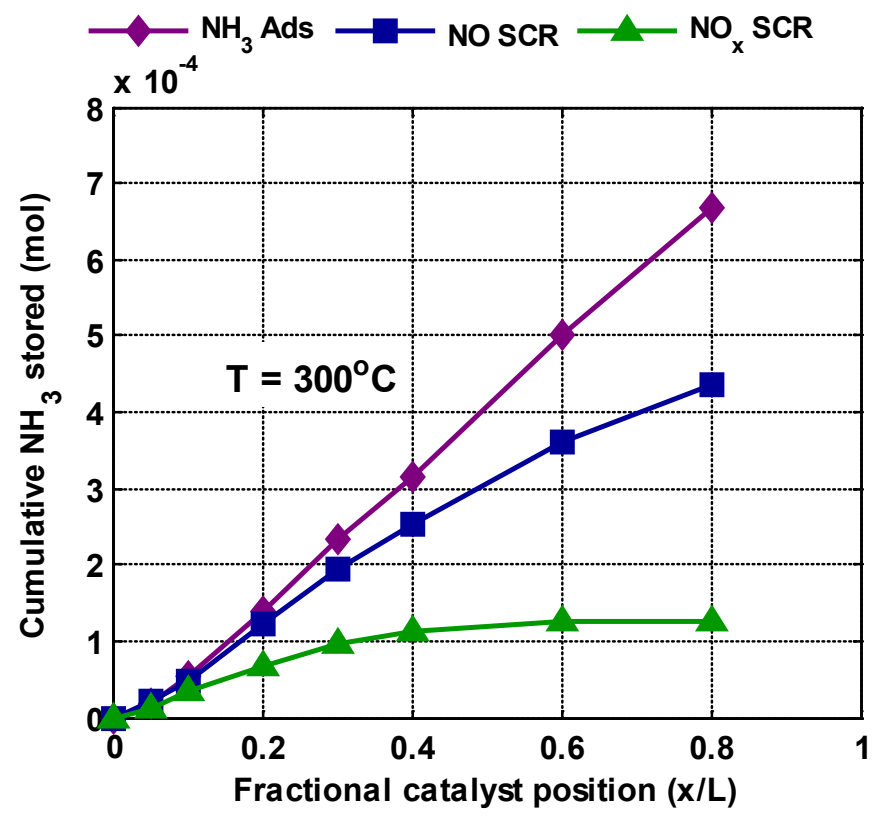

Figure F.5: $\mathrm{NH}_{3}$ storage as a function of axial position for the temperature of $300^{\circ} \mathrm{C}$ 


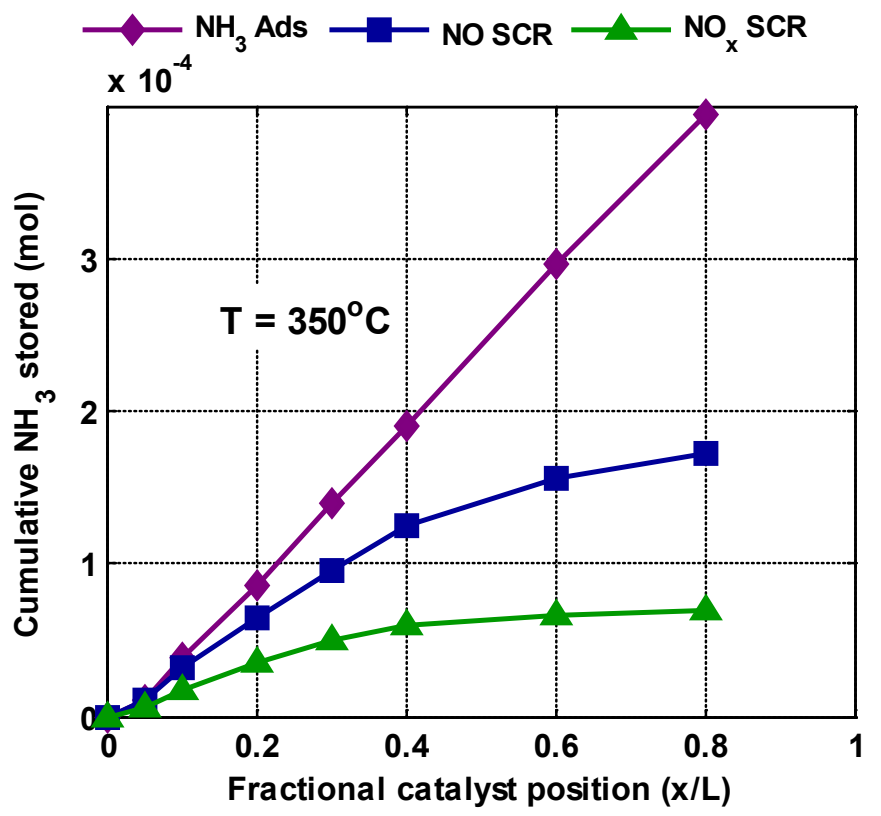

Figure F.6: $\mathrm{NH}_{3}$ storage as a function of axial position for the temperature of $350^{\circ} \mathrm{C}$

\section{Appendix G. Selection of Reactor Data for Model Calibration}

The reactor data showed abnormal SCR behavior at temperatures below $300{ }^{\circ} \mathrm{C}$. SCR outlet concentrations of $\mathrm{NH}_{3}, \mathrm{NO}, \mathrm{NO}_{2}$, and $\mathrm{N}_{2} \mathrm{O}$ measured by the FTIR at a temperature of $200^{\circ} \mathrm{C}$ and SV of $60 \mathrm{k} \mathrm{hr}^{-1}$ with corresponding inlet $\mathrm{NO}, \mathrm{NO}_{2}$, and $\mathrm{NH}_{3}$ is given in Figure G.1. An inhibition effect on the fast SCR reaction and slow SCR reaction is observed. The fast SCR section of the protocol with an ANR of 1.2 is shown in the plot between 90 and 300 minutes. It can be observed that $\mathrm{NO}_{\mathrm{x}}$ is completely reduced before 100 minutes right after $\mathrm{NH}_{3}$ was introduced into the gas stream. After a high $\mathrm{NO}_{\mathrm{x}}$ reduction period between 100 and 150 minutes, the $\mathrm{NO}_{\mathrm{x}}$ downstream of the SCR started to increase, meaning that the $\mathrm{NO}$ and $\mathrm{NO}_{2}$ reduction reactions were being inhibited. Finally, the catalyst reached an equilibrium state with stabilized $\mathrm{NH}_{3}$ slip and $\mathrm{NO}_{\mathrm{x}}$ downstream of the catalyst. The same inhibition effects were also observed when the 
ANR was switched to 1.0 between the time periods of 330 and 600 minutes. Greater inhibition effect on the SCR reactions were observed with the slow SCR sections of the protocol between about 780 and 1000 minutes. Only 20 percent of $\mathrm{NO}_{\mathrm{x}}$ was reduced when the catalyst reached the equilibrium state. No such effects were observed during the standard SCR reaction sections because $\mathrm{NO}_{x}$ reduction efficiency kept increasing with injection of $\mathrm{NH}_{3}$ before the catalyst reached the equilibrium state, as shown in the two sections with $\mathrm{NH}_{3}$ injection between 600 and 800 minutes. Since the inhibition only occurs when $\mathrm{NO}_{2}$ is in the feed gas, the most likely explanation is formation of $\mathrm{NH}_{4} \mathrm{NO}_{3}$ on the catalyst surface. $\mathrm{NH}_{4} \mathrm{NO}_{3}$ is stable at these low temperatures. As the surface loading of $\mathrm{NH}_{4} \mathrm{NO}_{3}$ increases, it could block active sites and/or pores in the zeolite, resulting in reduced catalytic activity. Similar phenomena was previously reported for $\mathrm{V}$ based and Fe-ZSM5 catalysts [49,134]. Reference [130] has proposed a model for $\mathrm{Cu}$ and Fe-zeolite SCR including this phenomena into the reaction mechanism. The formed $\mathrm{NH}_{4} \mathrm{NO}_{3}$ was considered to leave the catalyst in gaseous form instead of depositing on the catalyst surface. The model presented in this paper did not include the $\mathrm{NH}_{4} \mathrm{NO}_{3}$ formation and decomposition reactions and was not able to make similar predictions at these sections, the reactor data showed such phenomenon was not used for model calibration. The reactor data sets or sections from a specific data set not used for model calibration are shown in Table G.1.

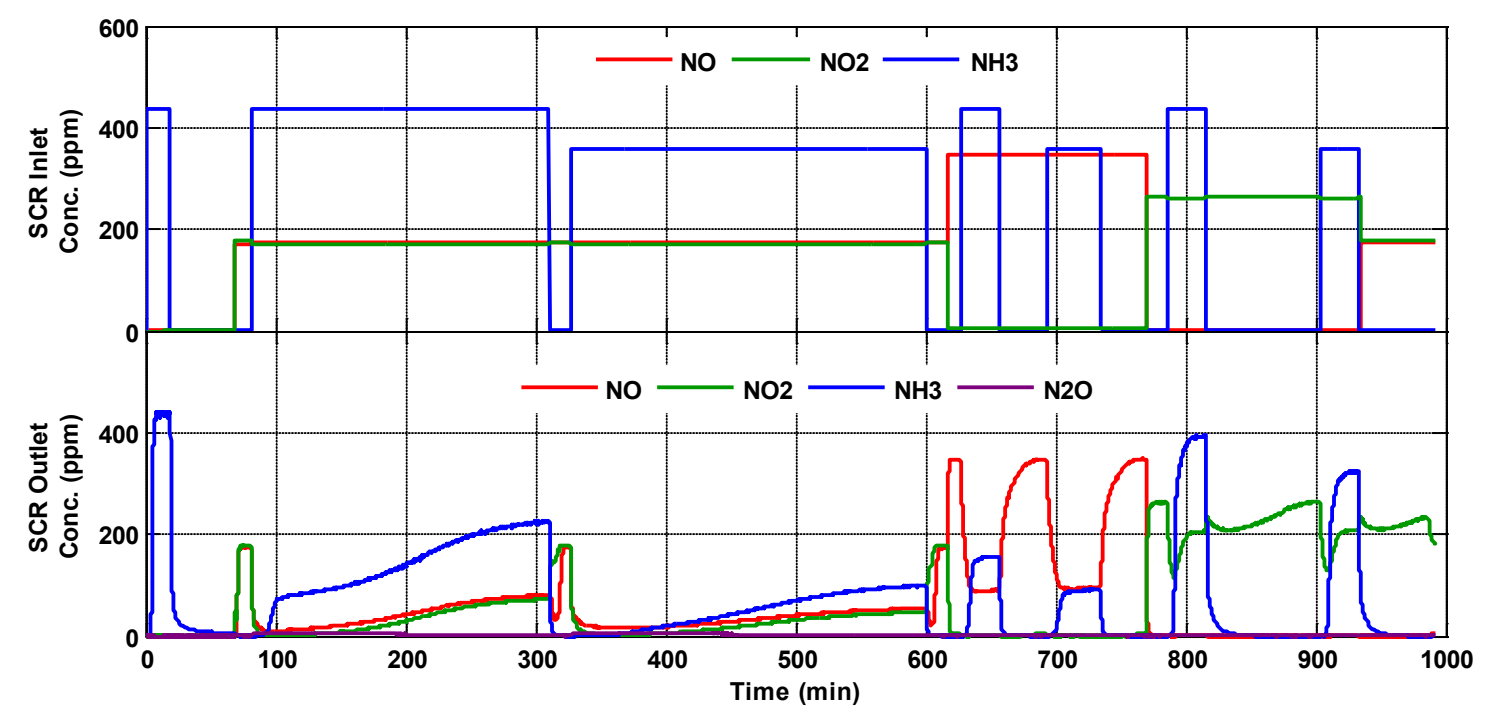

Figure G.1: SCR inlet and outlet concentrations for $200^{\circ} \mathrm{C}$ and $60 \mathrm{k} \mathrm{hr}^{-1} \mathrm{SV}$ 
Table G.1: Reactor experimental data sets with marked steps $(x)$ that were not used for model calibration

\begin{tabular}{|c|c|c|c|c|c|c|c|c|c|c|c|c|c|c|c|c|c|c|c|c|c|}
\hline \multirow{2}{*}{ SV } & \multirow{2}{*}{$\begin{array}{c}\text { Temp. } \\
\left({ }^{\circ} \mathrm{C}\right)\end{array}$} & \multicolumn{20}{|c|}{ Step Number } \\
\hline & & 1 & 2 & 3 & 4 & 5 & 6 & 7 & 8 & 9 & 10 & 11 & 12 & 13 & 14 & 15 & 16 & 17 & 18 & 19 & 20 \\
\hline \multirow{5}{*}{$\begin{array}{l}60 \mathrm{k} \\
1 / \mathrm{hr}\end{array}$} & 200 & & & & & & $x$ & $x$ & $x$ & $x$ & & & & & & & $x$ & $x$ & $x$ & $x$ & $x$ \\
\hline & 250 & & & & & & & & & & & & & & & & $x$ & $x$ & $x$ & $x$ & $x$ \\
\hline & 300 & & & & & & & & & & & & & & & & & & & & \\
\hline & 350 & & & & & & & & & & & & & & & & & & & & \\
\hline & 400 & & & & & & & & & & & & & & & & & & & & \\
\hline \multirow{10}{*}{$\begin{array}{l}90 \mathrm{k} \\
1 / \mathrm{hr}\end{array}$} & 200 & & & & & & $x$ & $x$ & $x$ & $x$ & & & & & & & $x$ & $x$ & $x$ & $x$ & $x$ \\
\hline & 225 & & & & & & & & & & & & & & & & $\times$ & $x$ & $x$ & $\times$ & $x$ \\
\hline & 250 & & & & & & & & & & & & & & & & $x$ & $x$ & $x$ & $x$ & $x$ \\
\hline & 275 & & & & & & & & & & & & & & & & $x$ & $x$ & $x$ & $x$ & $x$ \\
\hline & 300 & & & & & & & & & & & & & & & & $x$ & $x$ & $x$ & $x$ & $x$ \\
\hline & 325 & & & & & & & & & & & & & & & & & & & & \\
\hline & 350 & & & & & & & & & & & & & & & & & & & & \\
\hline & 375 & & & & & & & & & & & & & & & & & & & & \\
\hline & 400 & & & & & & & & & & & & & & & & & & & & \\
\hline & 425 & & & & & & & & & & & & & & & & & & & & \\
\hline \multirow{5}{*}{$\begin{array}{l}120 \mathrm{k} \\
1 / \mathrm{hr}\end{array}$} & 200 & & & & & & $x$ & $x$ & $x$ & $x$ & & & & & & & $x$ & $x$ & $x$ & $x$ & $x$ \\
\hline & 250 & & & & & & & & & & & & & & & & $x$ & $x$ & $x$ & $x$ & $x$ \\
\hline & 300 & & & & & & & & & & & & & & & & $x$ & $x$ & $x$ & $x$ & $x$ \\
\hline & 350 & & & & & & & & & & & & & & & & & & & & \\
\hline & 400 & & & & & & & & & & & & & & & & & & & & \\
\hline
\end{tabular}




\section{Appendix H. Simulation Results of TPD Experiments}

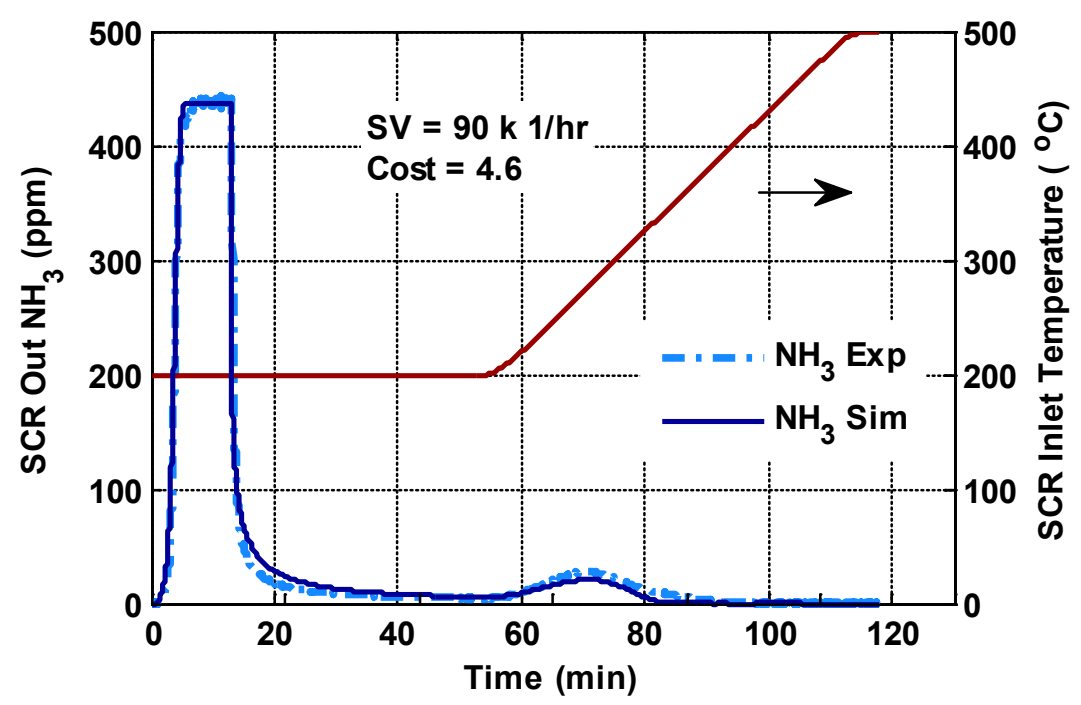

Figure H.1: Comparison of SCR outlet $\mathrm{NH}_{3}$ between simulation and experimental results for the space velocity of $90,000 \mathrm{hr}^{-1}$

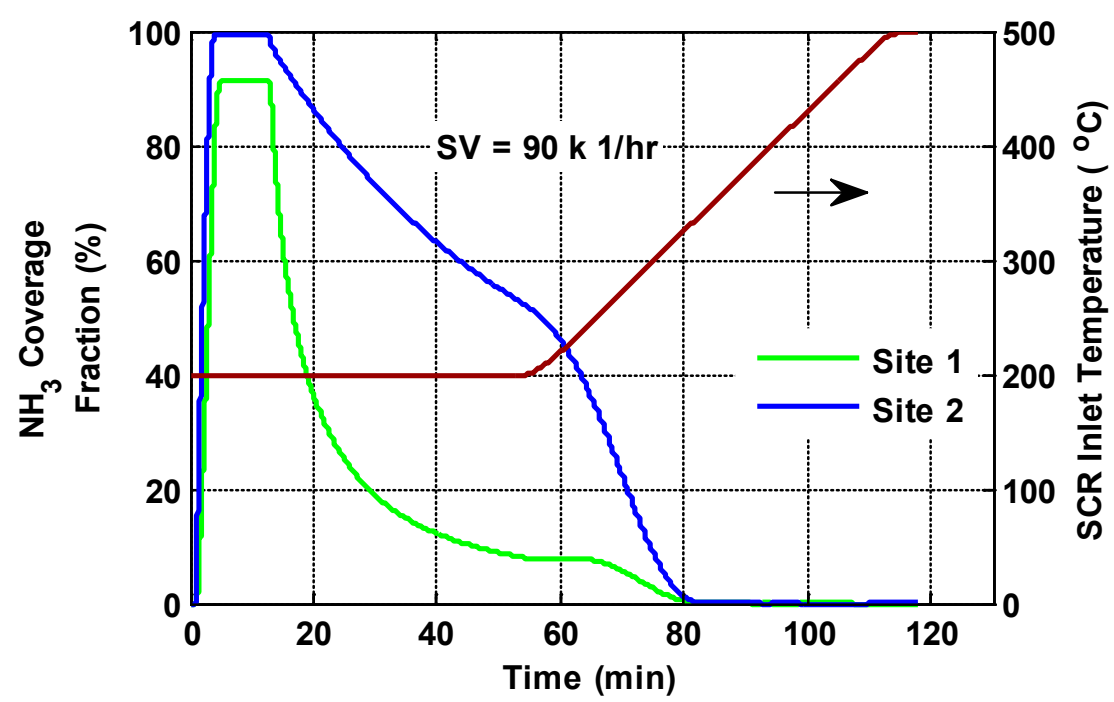

Figure H.2: Simulated $\mathrm{NH}_{3}$ coverage fraction for TPD test for the space velocity of $90,000 \mathrm{hr}^{-1}$ 


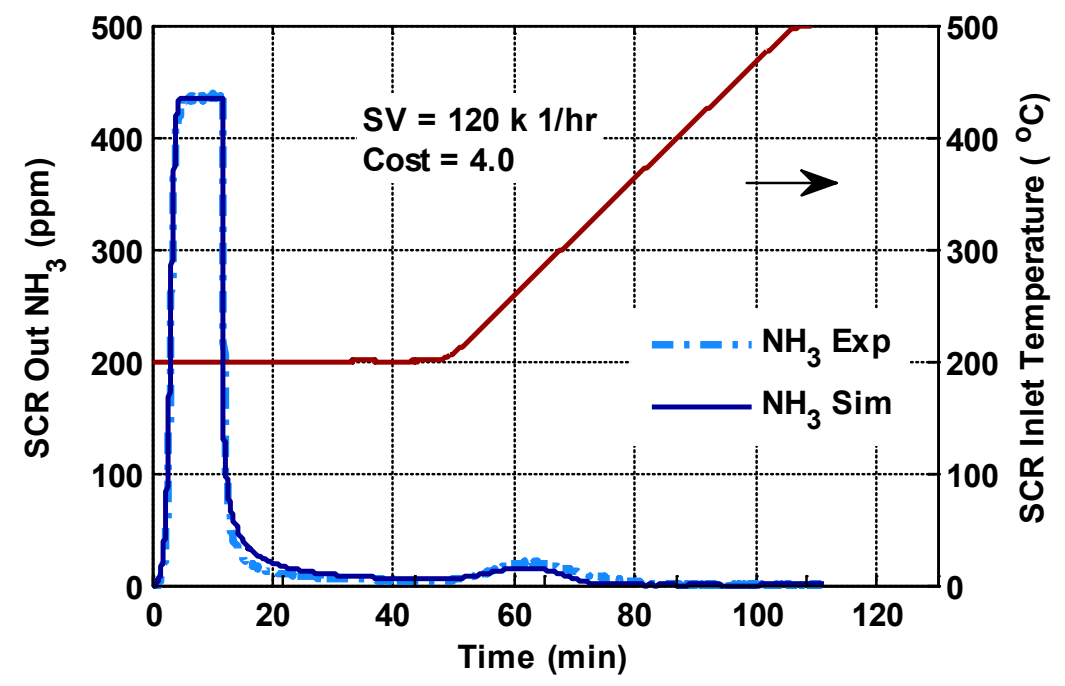

Figure H.3: Comparison of SCR outlet $\mathrm{NH}_{3}$ between simulation and experimental results for the space velocity of $120,000 \mathrm{hr}^{-1}$

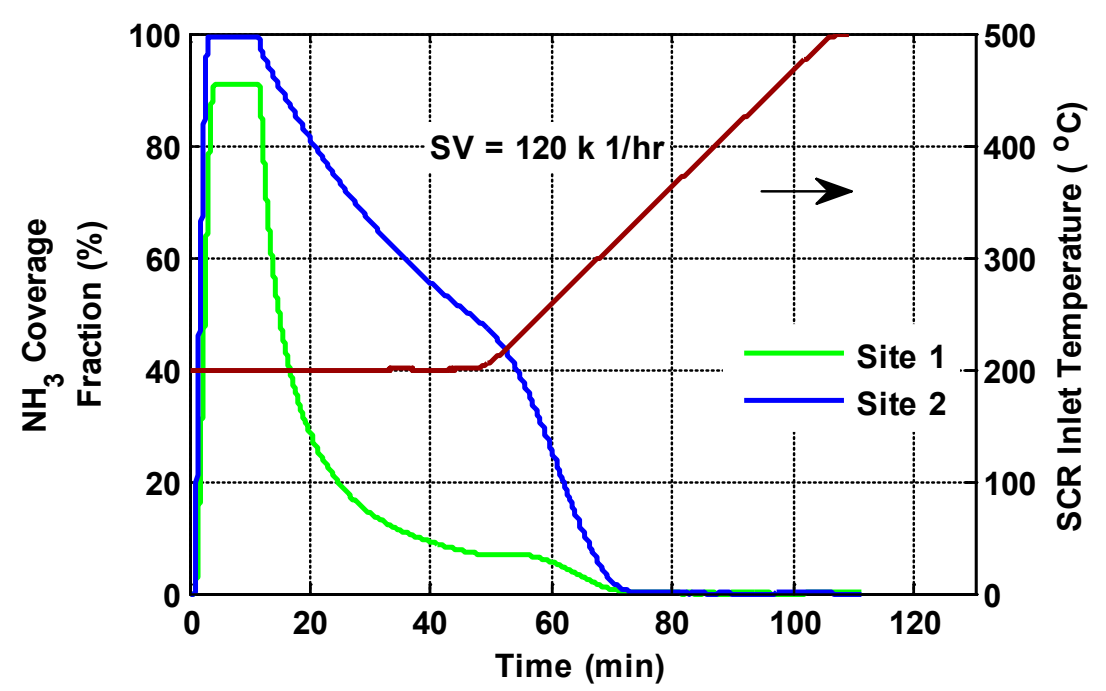

Figure H.4: Simulated $\mathrm{NH}_{3}$ coverage fraction for TPD test for the space velocity of $120,000 \mathrm{hr}^{-1}$ 


\section{Appendix I.Simulation Results of Isothermal Reactor Tests}

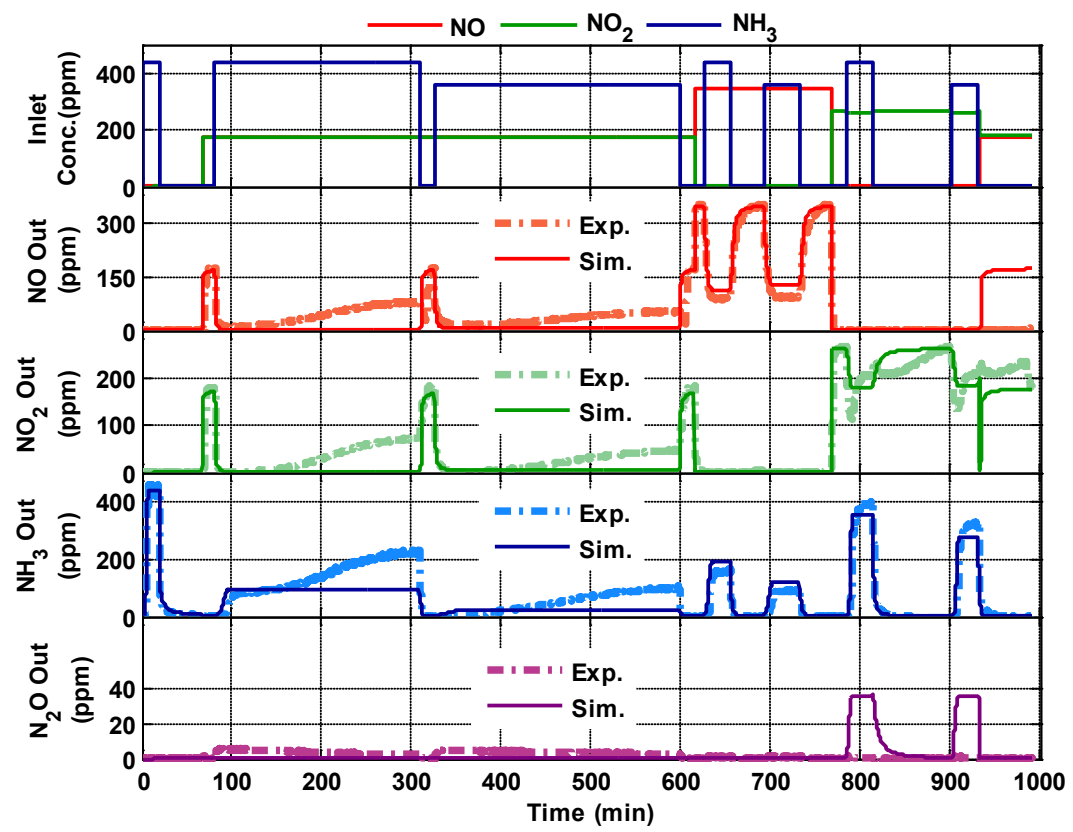

Figure I.1: Model simulation vs. reactor data, SV $=60 \mathrm{k} / \mathrm{hr}, \mathrm{Temp} .=200^{\circ} \mathrm{C}$

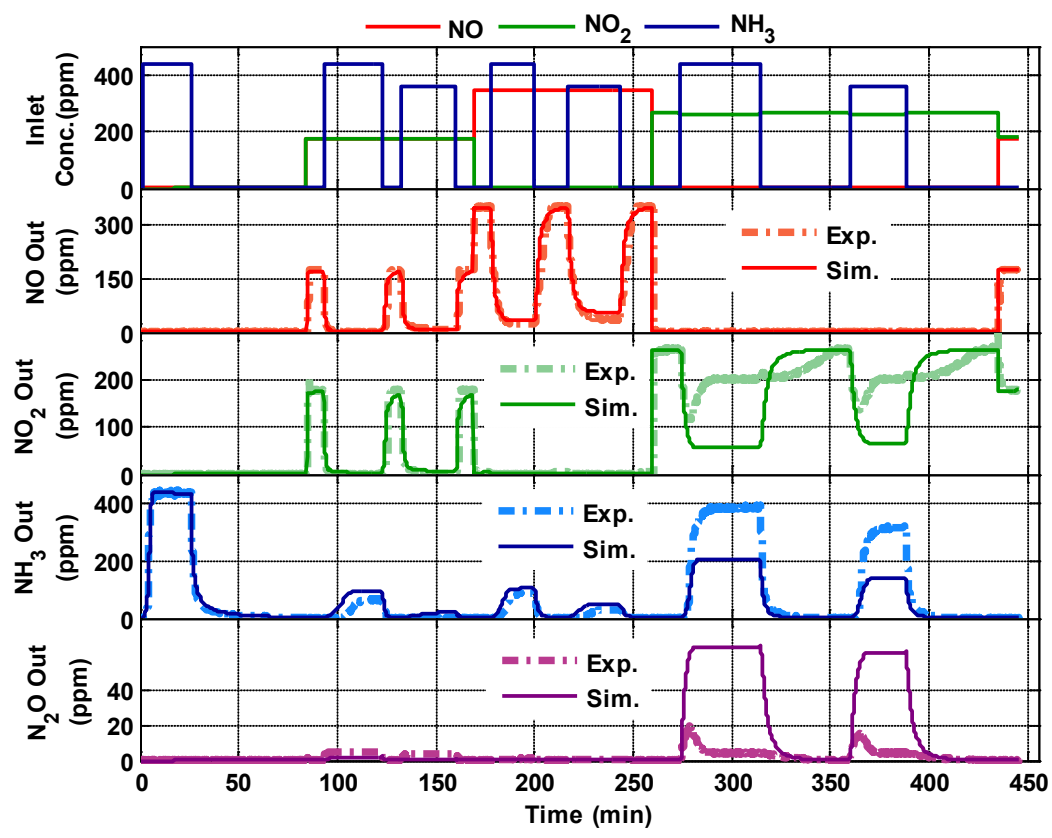

Figure I.2: Model simulation vs. reactor data, $\mathrm{SV}=60 \mathrm{k} / \mathrm{hr}, \mathrm{Temp} .=250^{\circ} \mathrm{C}$ 


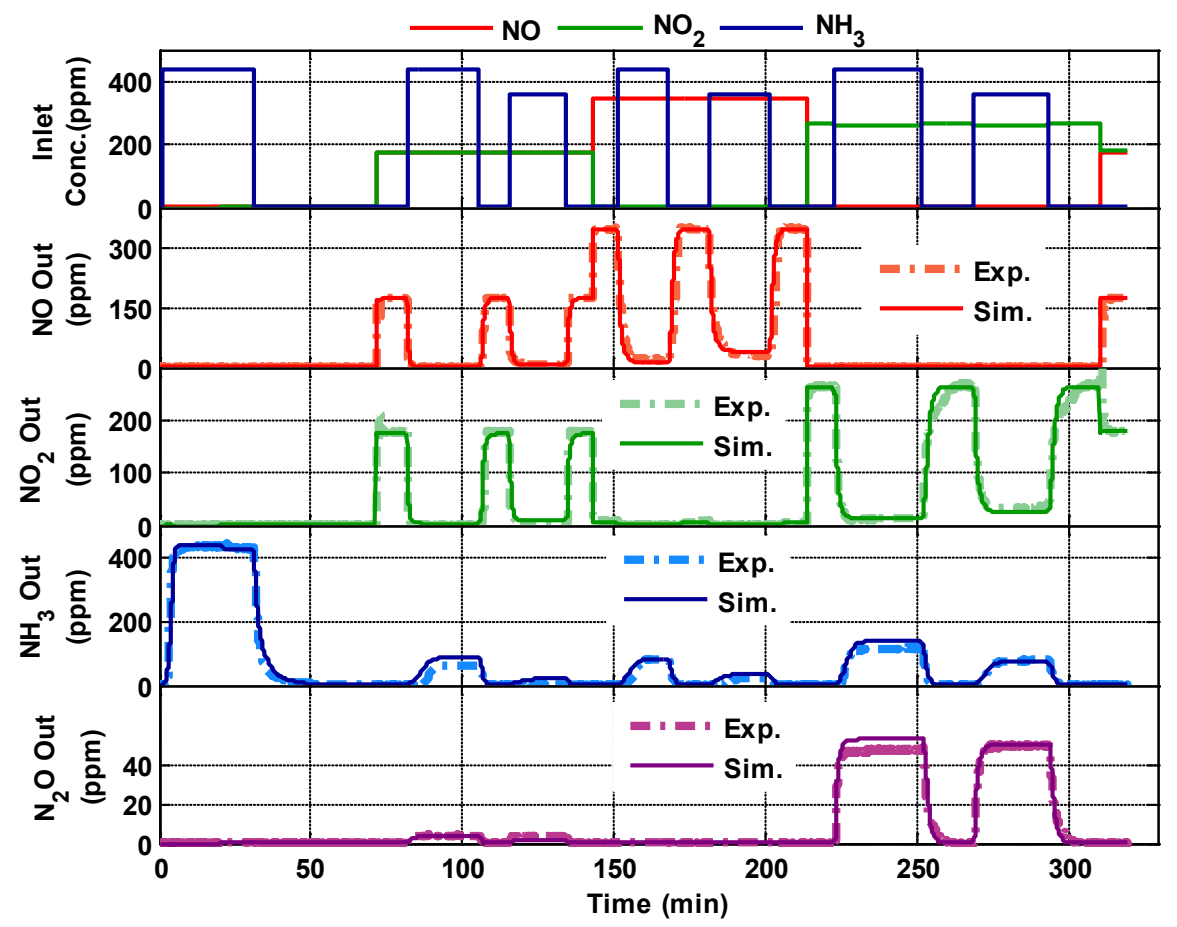

Figure I.3: Model simulation vs. reactor data, SV $=60 \mathrm{k} / \mathrm{hr}$, Temp. $=300^{\circ} \mathrm{C}$

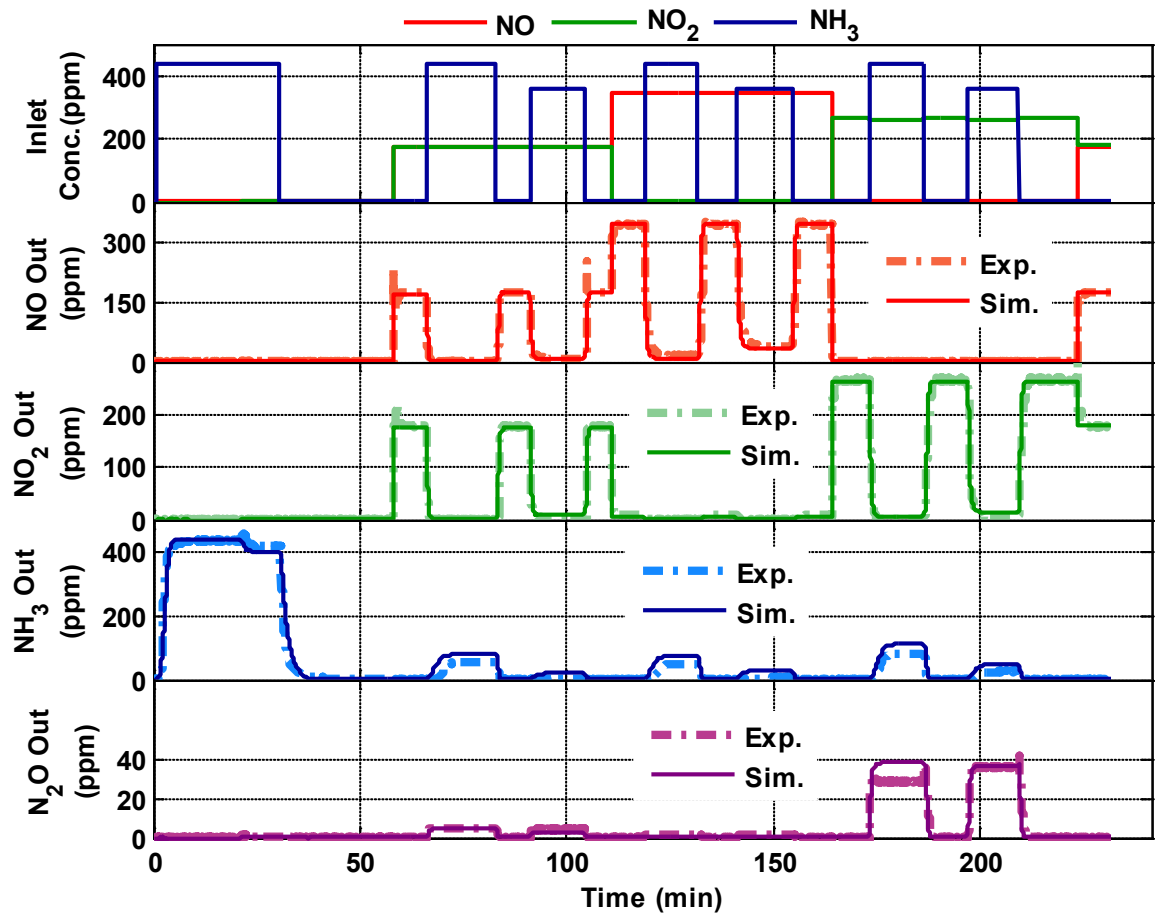

Figure I.4: Model simulation vs. reactor data, SV $=60 \mathrm{k} / \mathrm{hr}, \mathrm{Temp} .=350^{\circ} \mathrm{C}$ 


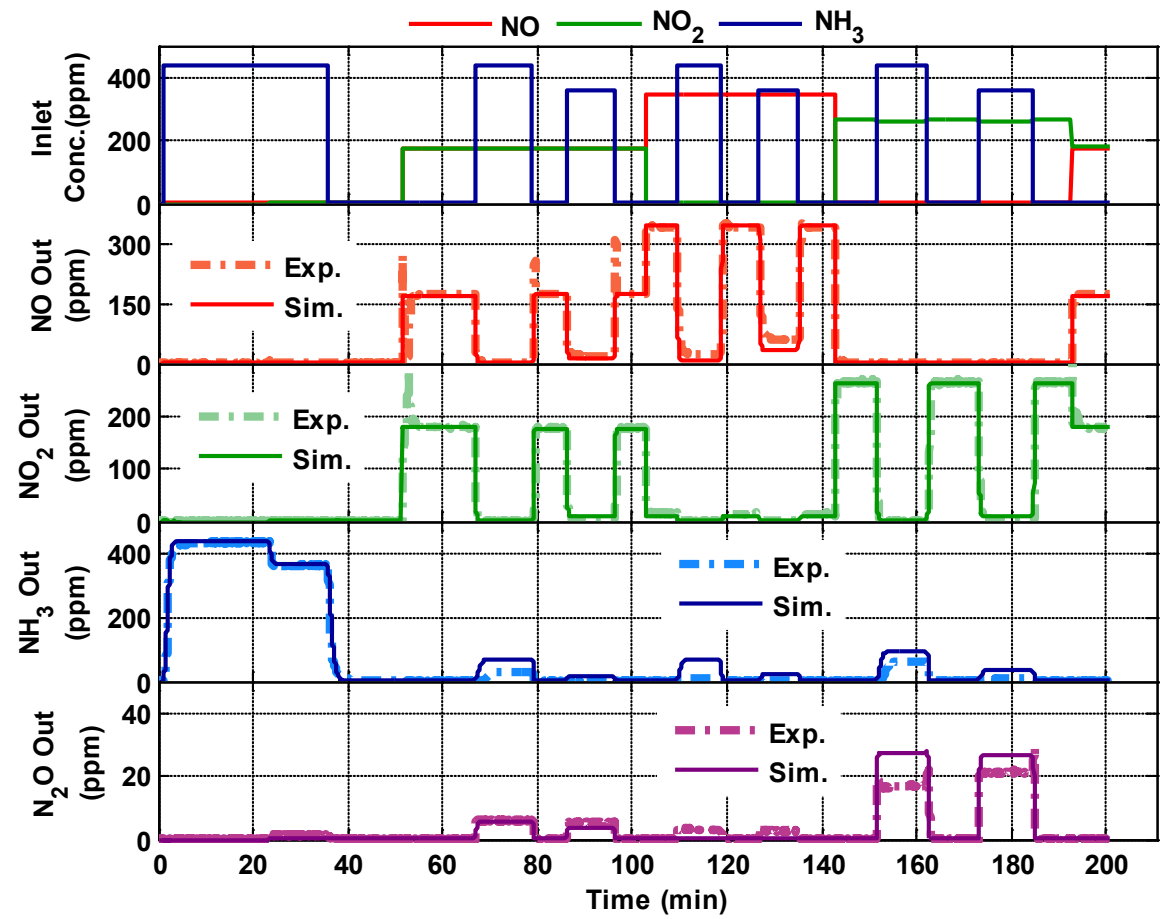

Figure I.5: Model simulation vs. reactor data, SV $=60 \mathrm{k} / \mathrm{hr}$, Temp. $=400^{\circ} \mathrm{C}$

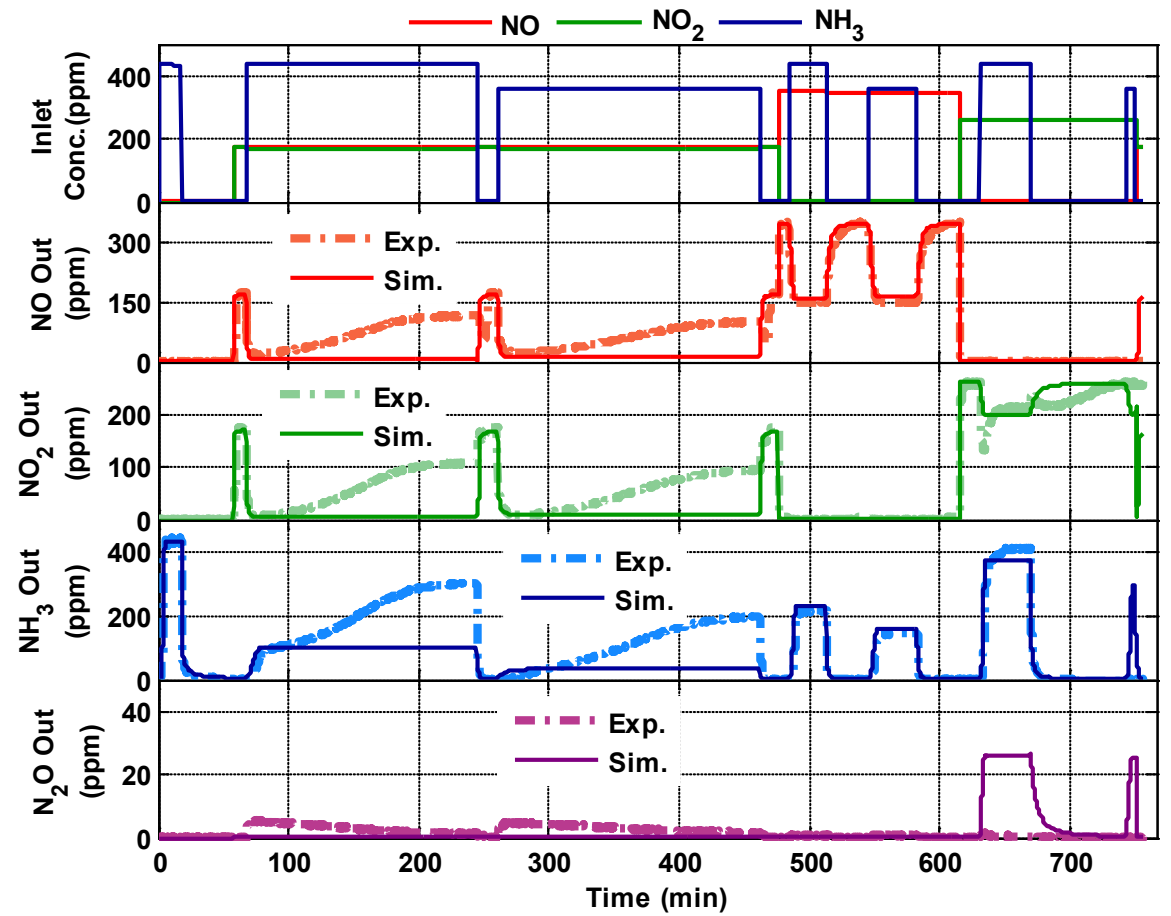

Figure I.6: Model simulation vs. reactor data, SV $=90 \mathrm{k} / \mathrm{hr}, \mathrm{Temp} .=200^{\circ} \mathrm{C}$ 


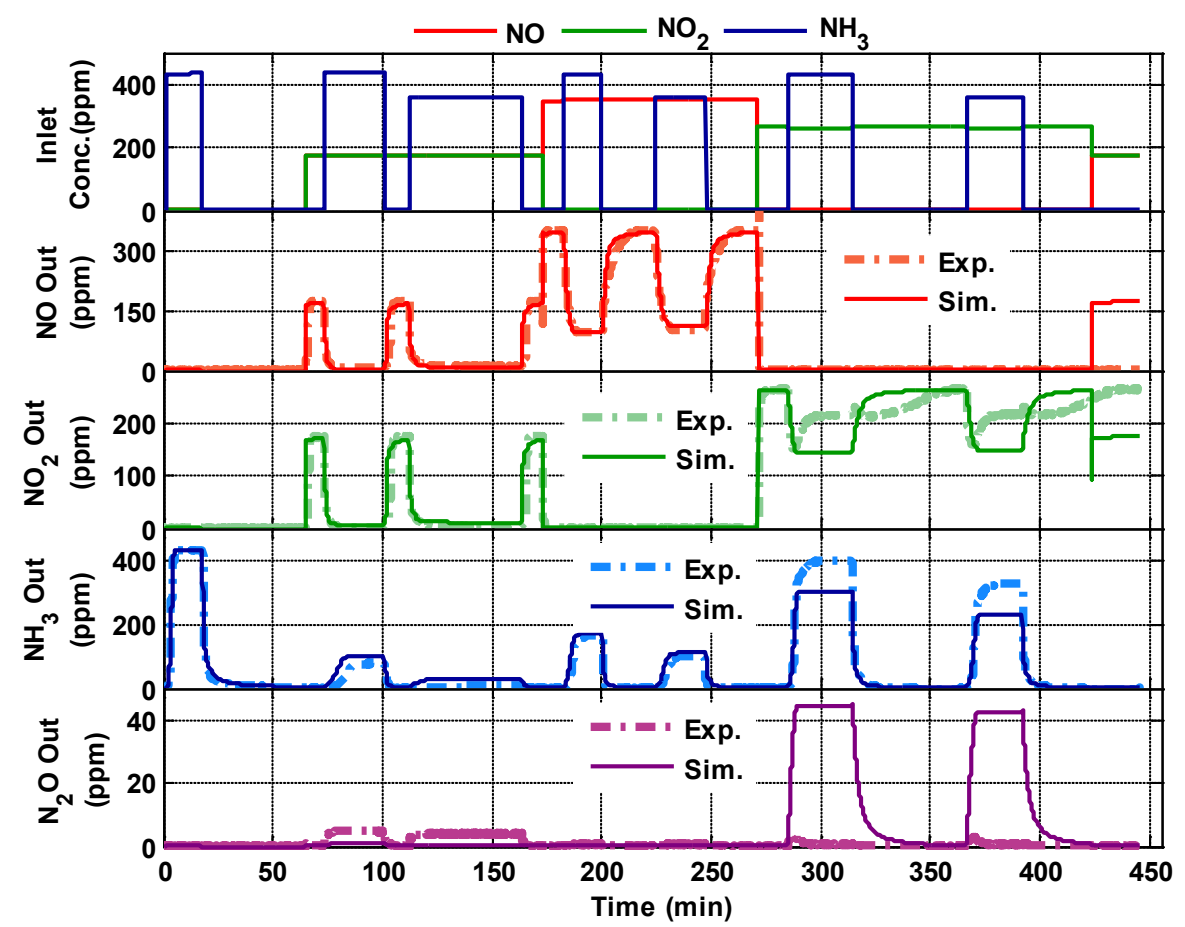

Figure I.7: Model simulation vs. reactor data, SV $=90 \mathrm{k} / \mathrm{hr}$, Temp. $=225^{\circ} \mathrm{C}$

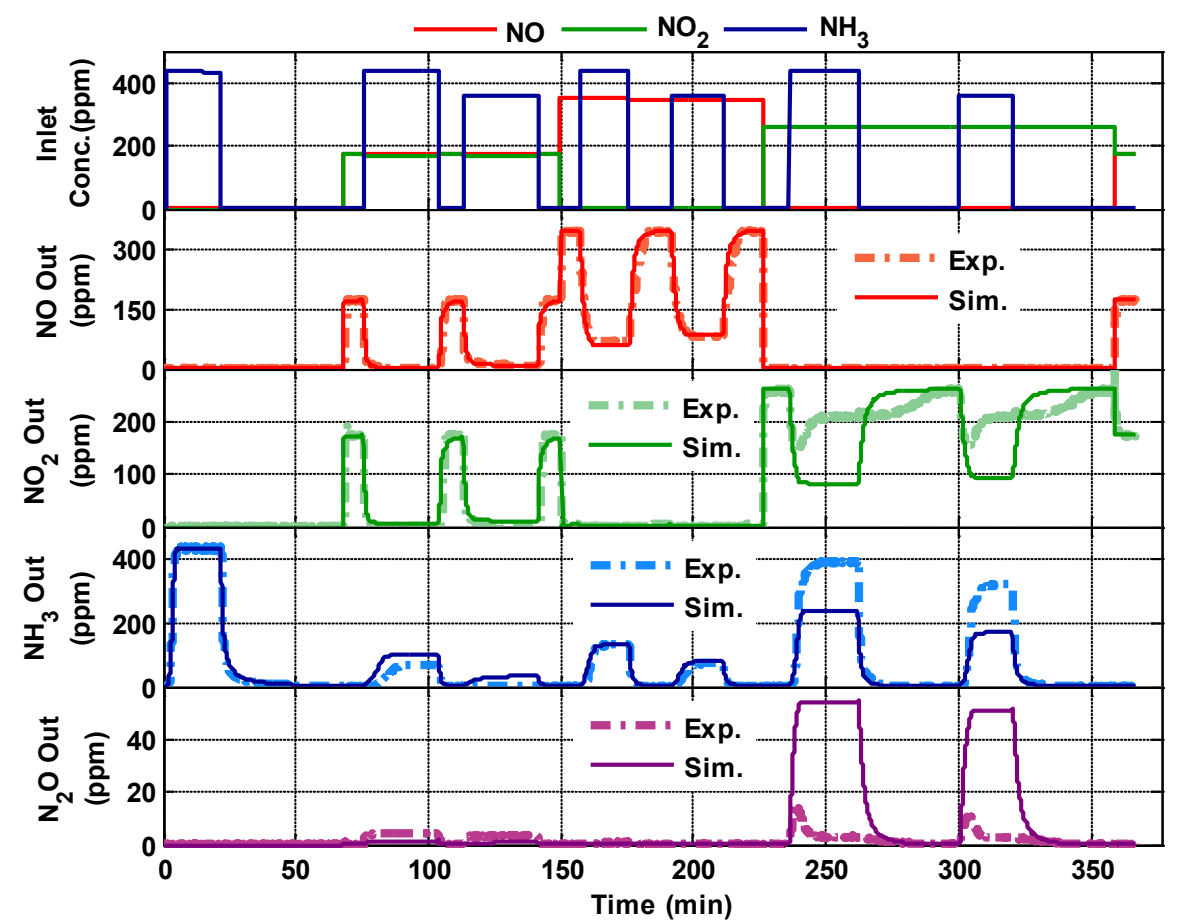

Figure I.8: Model simulation vs. reactor data, SV $=90 \mathrm{k} / \mathrm{hr}$, Temp. $=250^{\circ} \mathrm{C}$ 


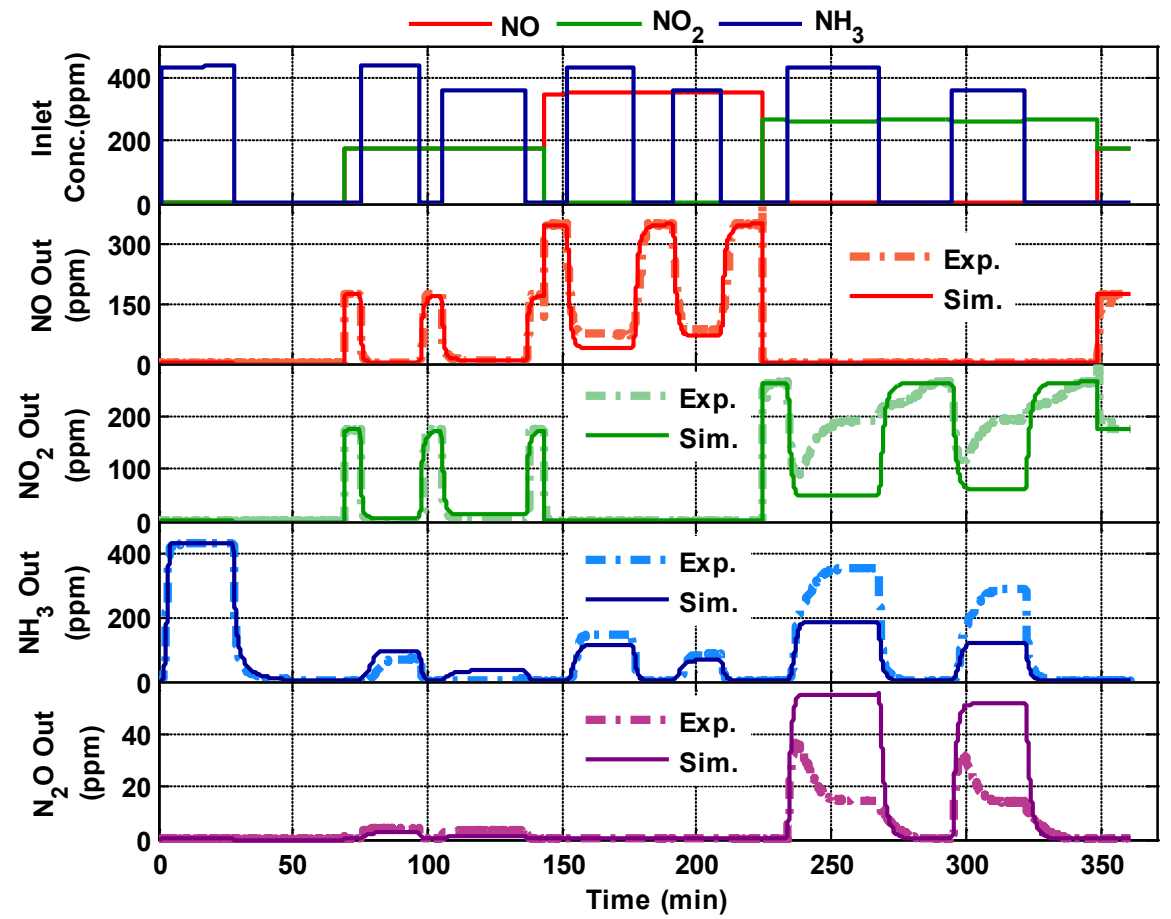

Figure I.9: Model simulation vs. reactor data, SV $=90 \mathrm{k} / \mathrm{hr}$, Temp. $=275^{\circ} \mathrm{C}$

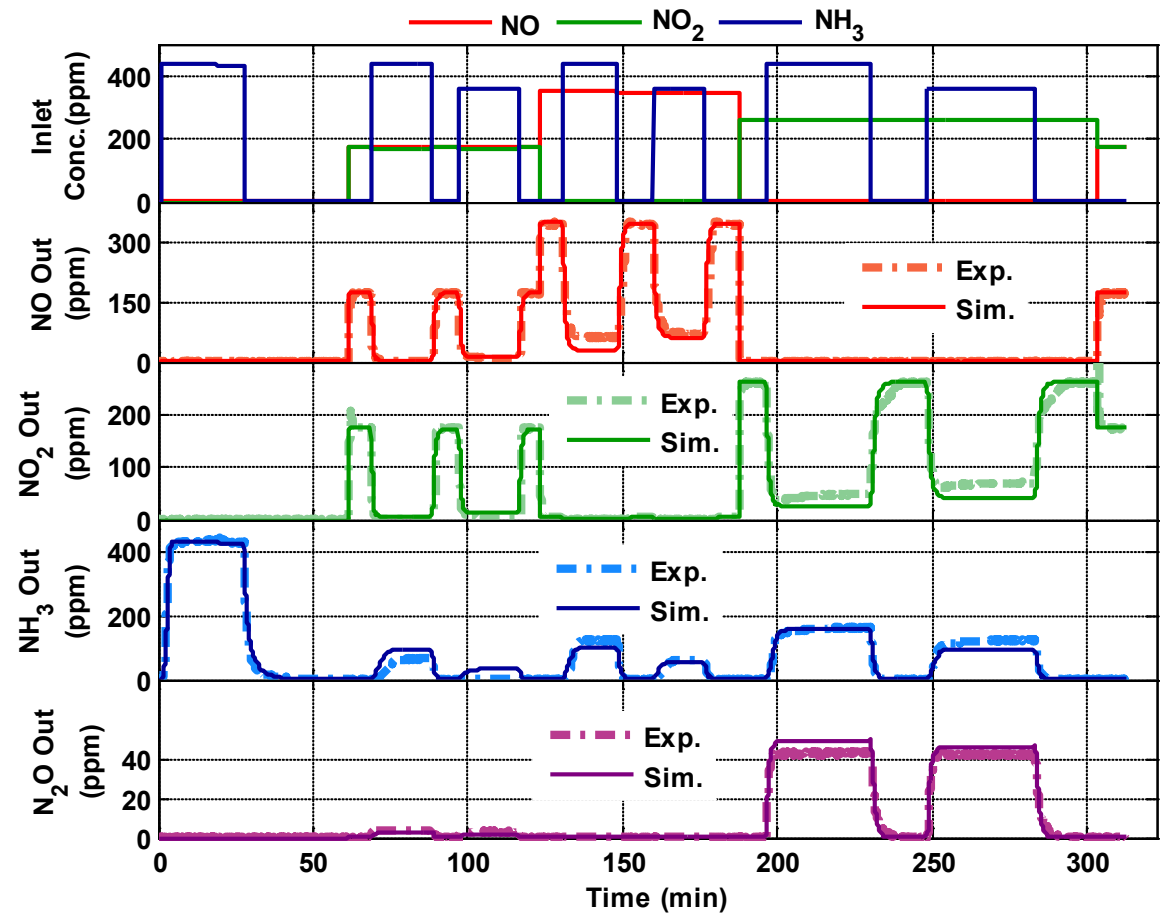

Figure I.10: Model simulation vs. reactor data, SV $=90 \mathrm{k} / \mathrm{hr}$, Temp. $=300^{\circ} \mathrm{C}$ 


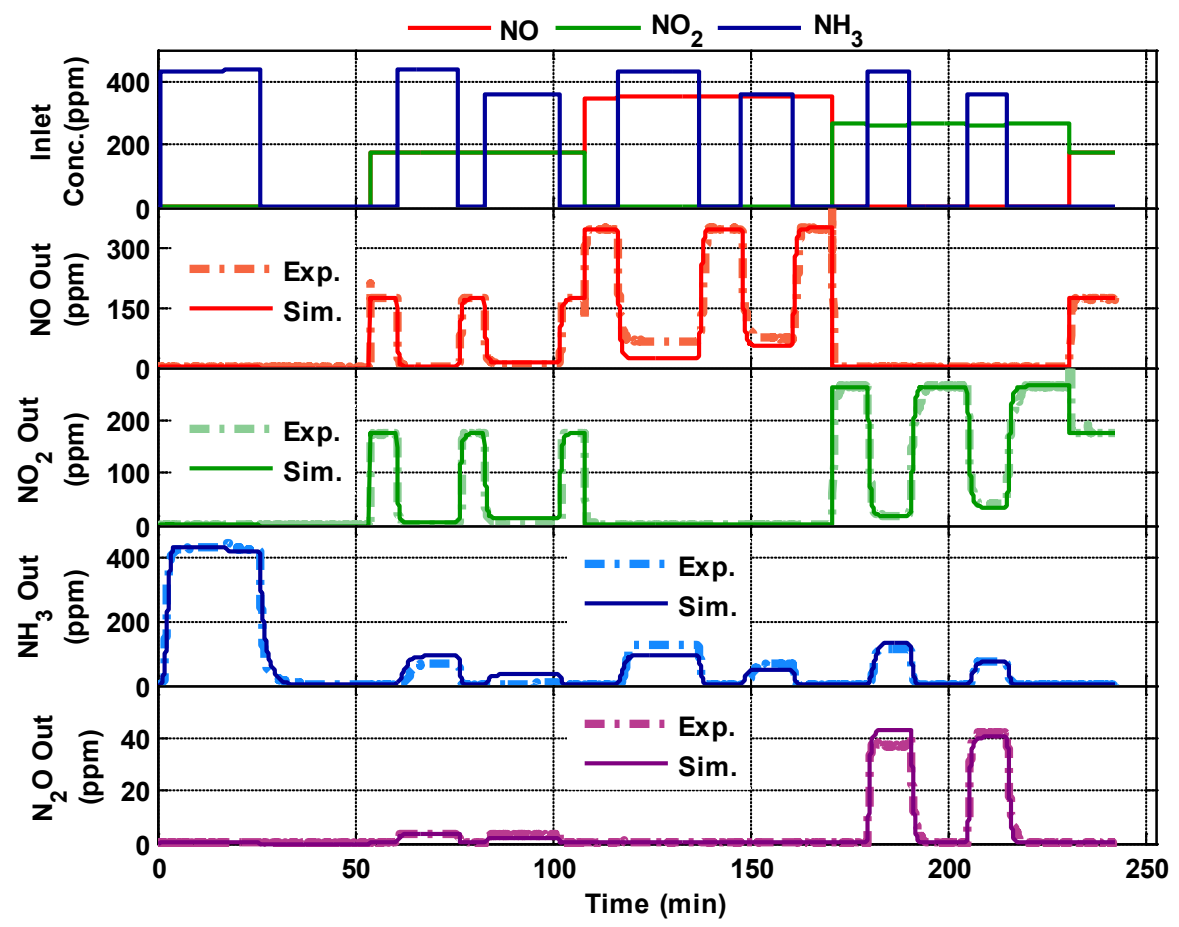

Figure I.11: Model simulation vs. reactor data, $\mathrm{SV}=90 \mathrm{k} / \mathrm{hr}, \mathrm{Temp} .=325^{\circ} \mathrm{C}$

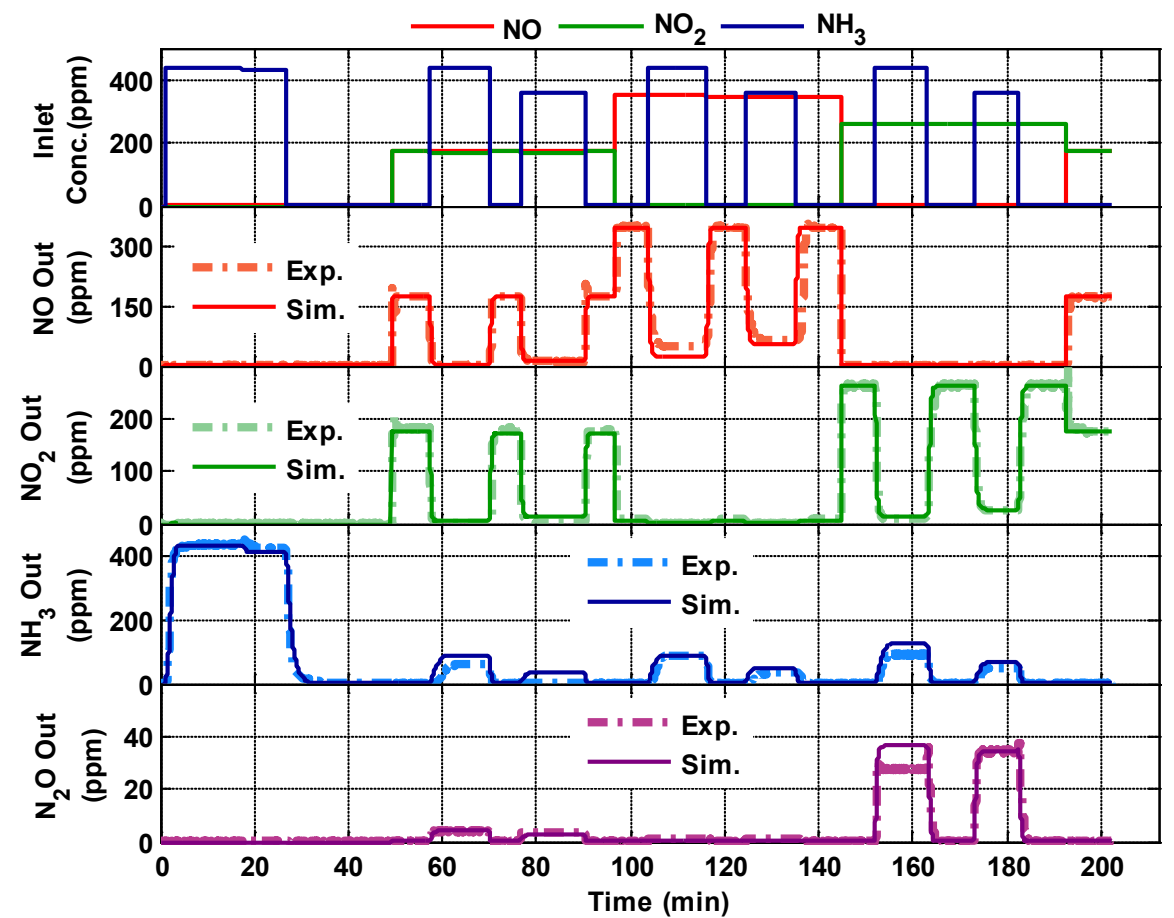

Figure I.12: Model simulation vs. reactor data, SV $=90 \mathrm{k} / \mathrm{hr}, \mathrm{Temp} .=350^{\circ} \mathrm{C}$ 


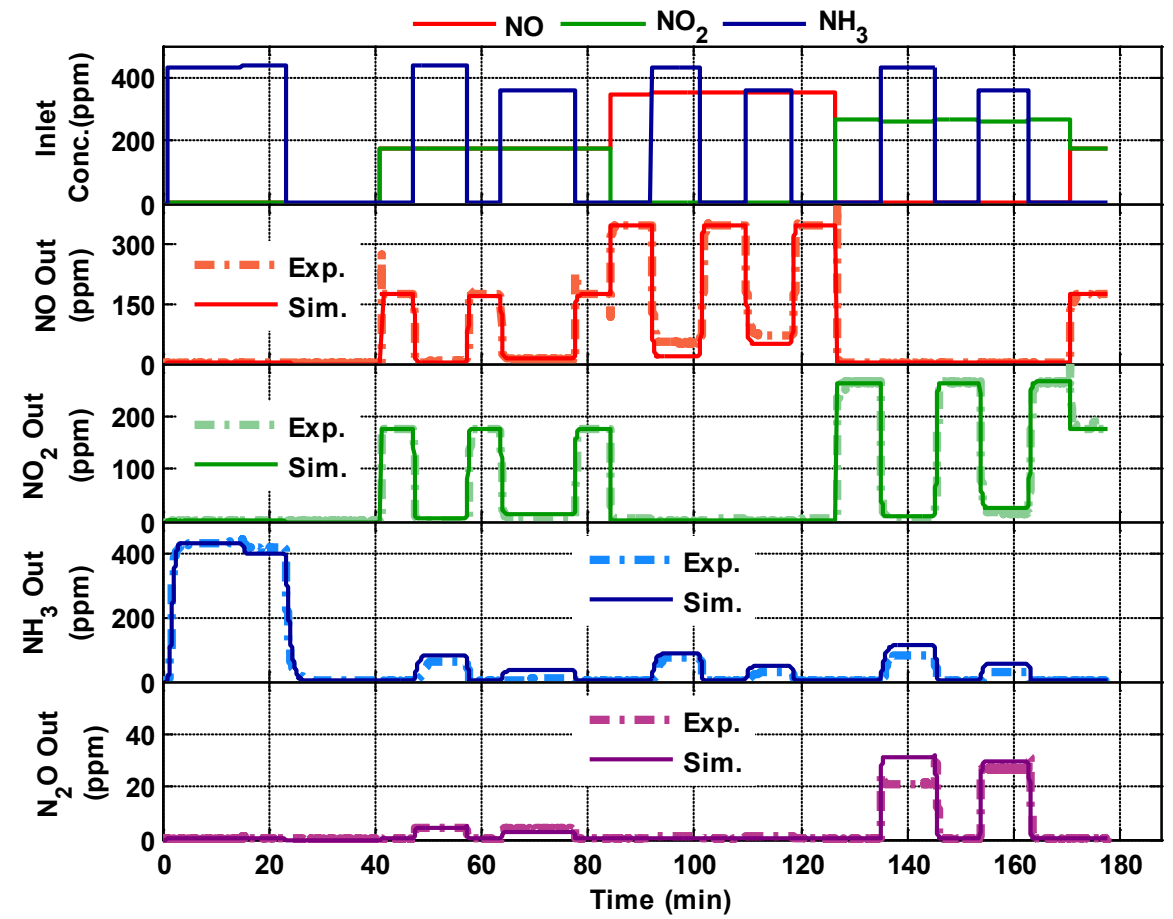

Figure I.13: Model simulation vs. reactor data, SV $=90 \mathrm{k} / \mathrm{hr}$, Temp. $=375^{\circ} \mathrm{C}$

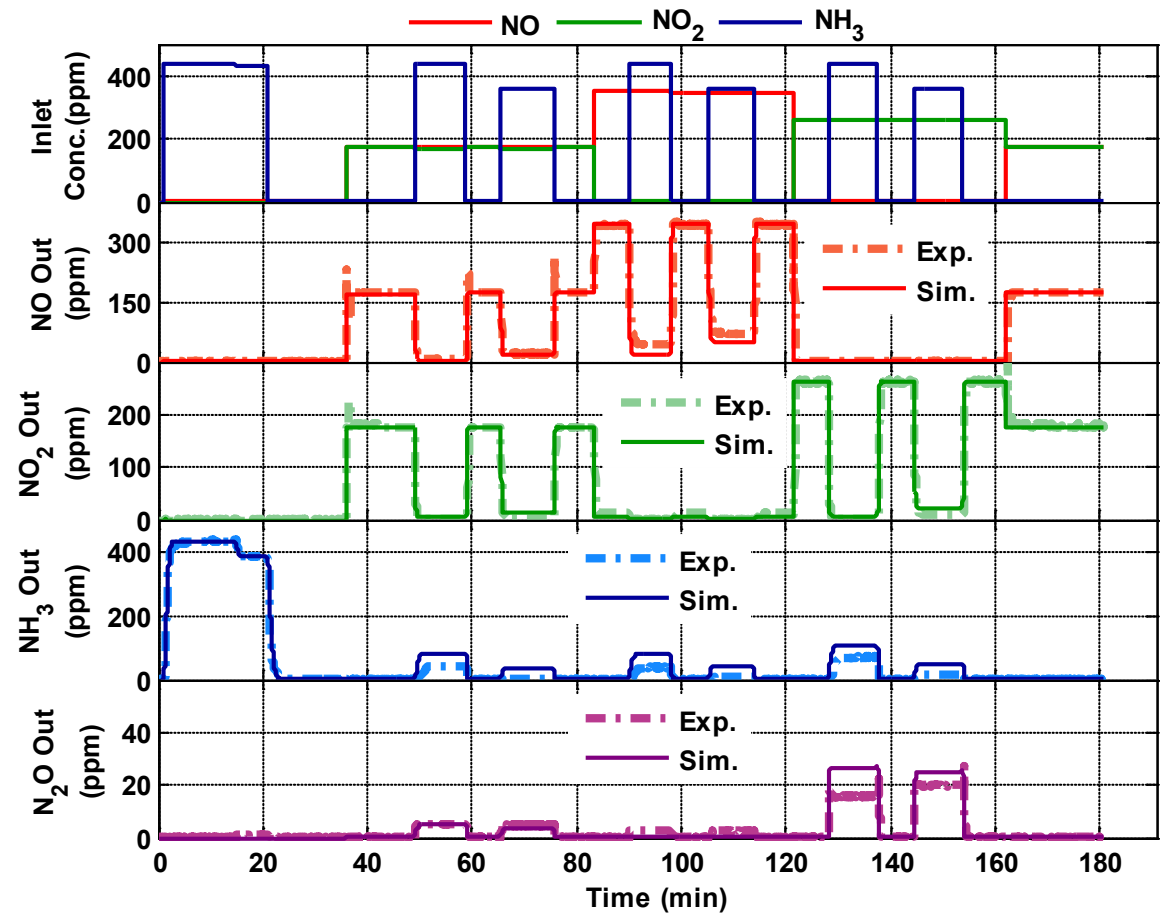

Figure I.14: Model simulation vs. reactor data, SV $=90 \mathrm{k} / \mathrm{hr}$, Temp. $=400^{\circ} \mathrm{C}$ 


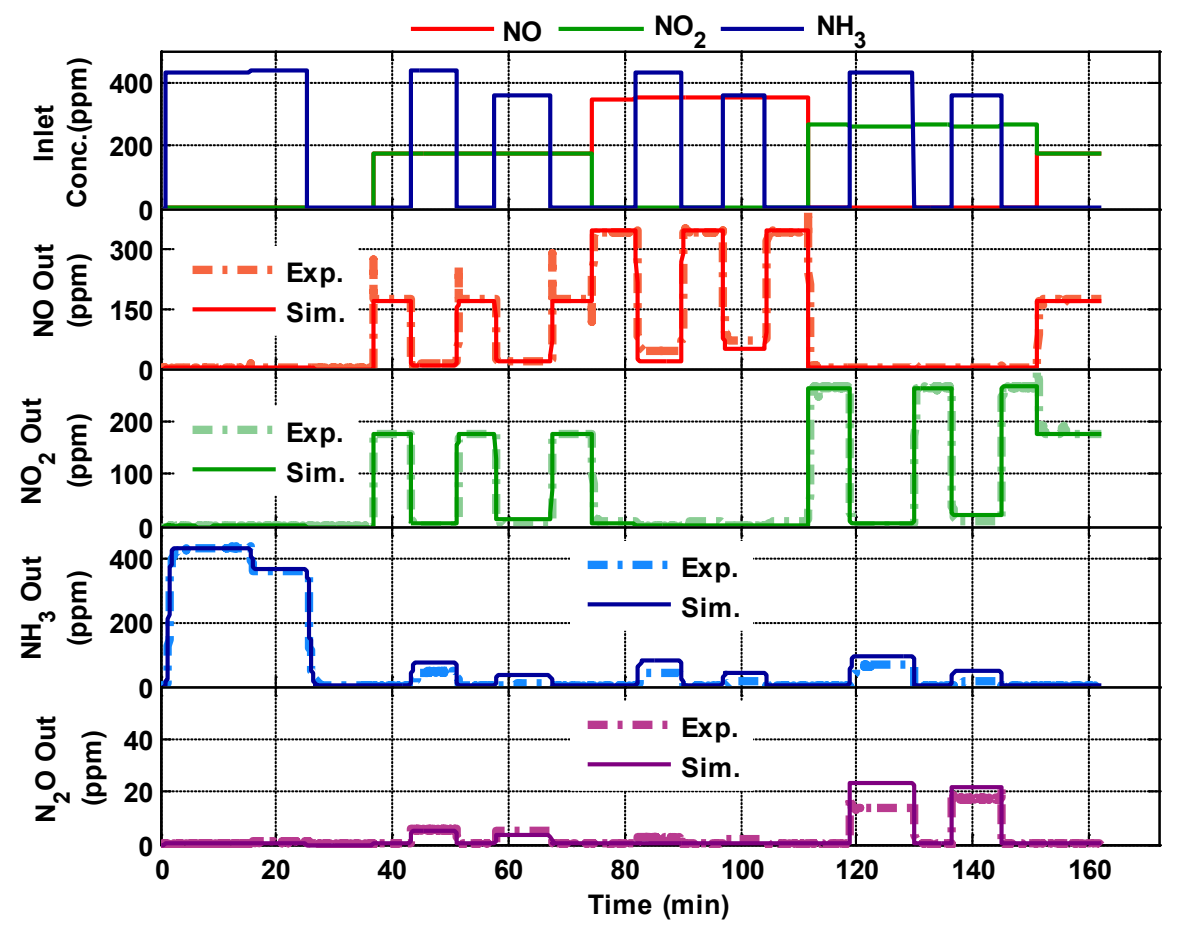

Figure I.15: Model simulation vs. reactor data, SV $=90 \mathrm{k} / \mathrm{hr}$, Temp. $=425^{\circ} \mathrm{C}$

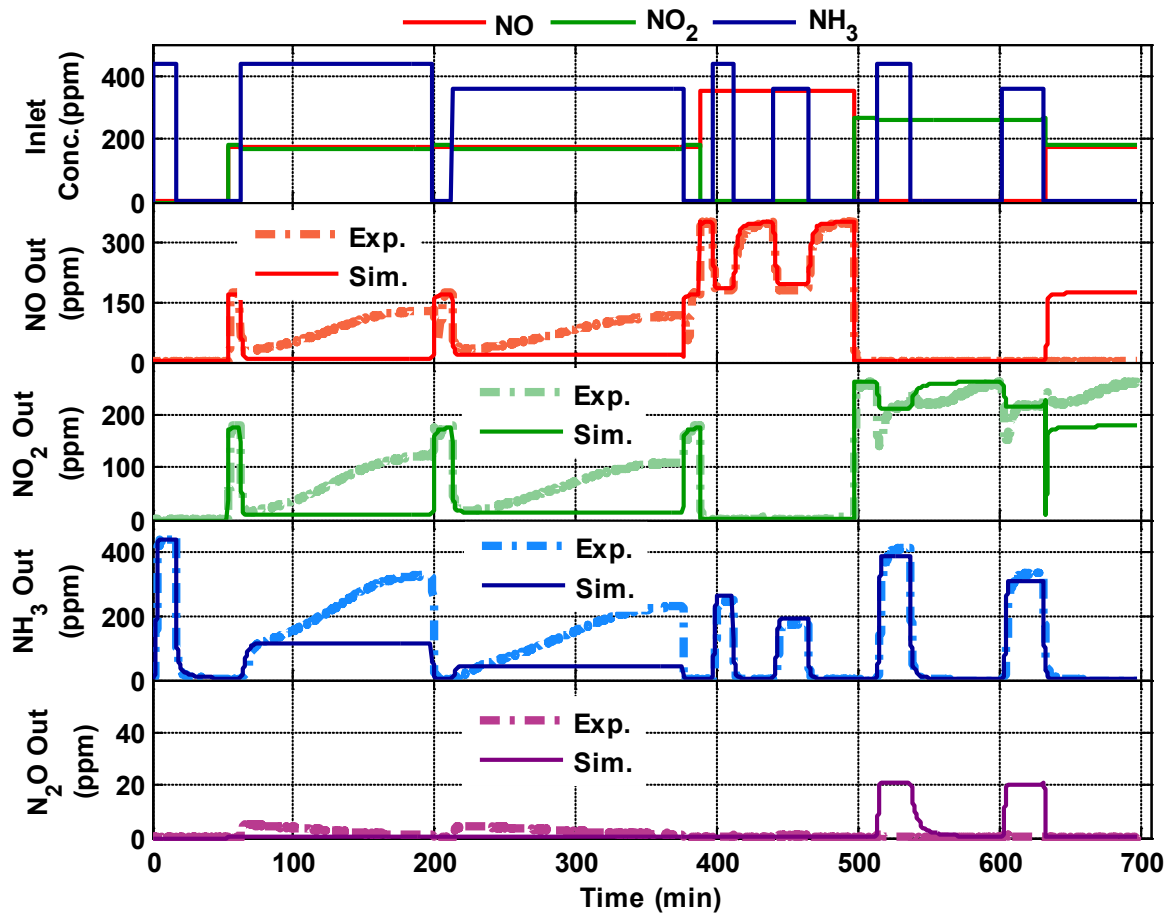

Figure I.16: Model simulation vs. reactor data, SV $=120 \mathrm{k} / \mathrm{hr}$, Temp. $=200^{\circ} \mathrm{C}$ 


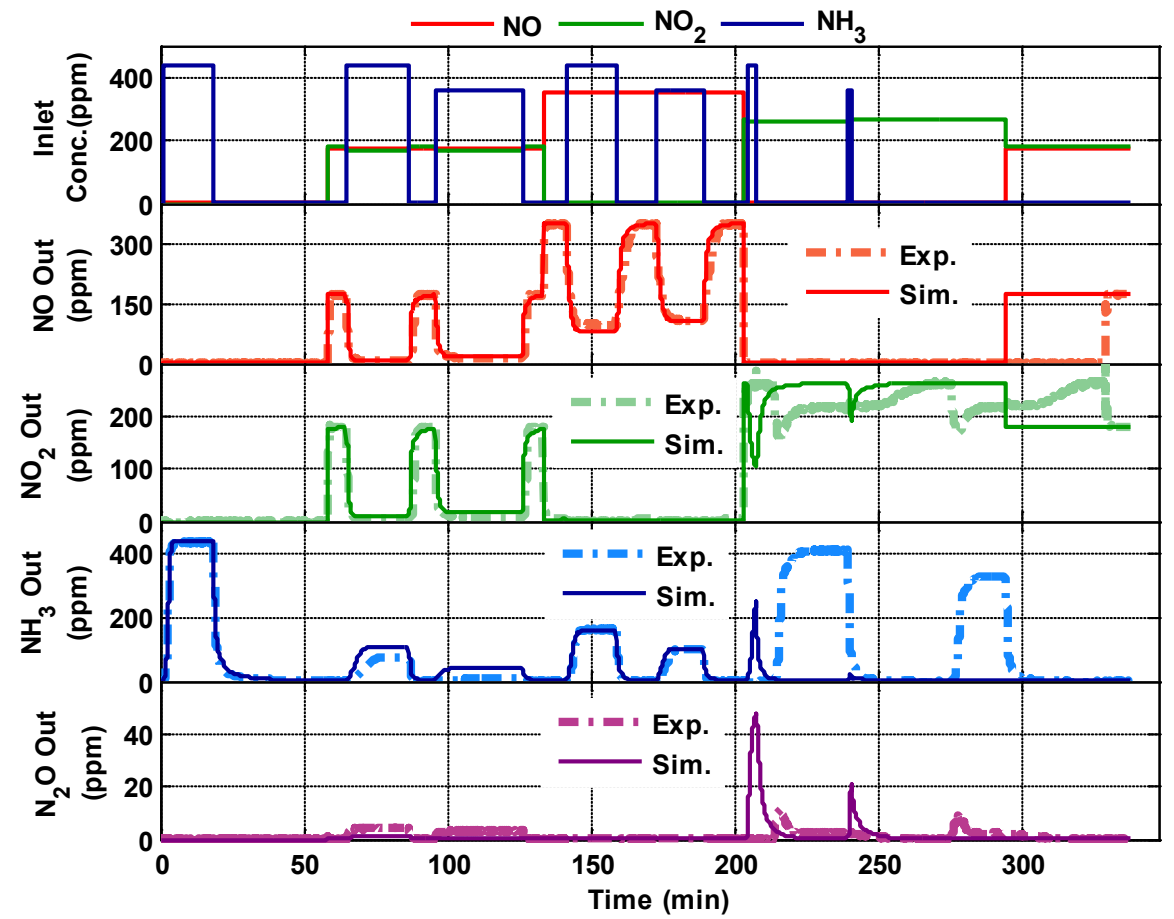

Figure I.17: Model simulation vs. reactor data, SV $=120 \mathrm{k} / \mathrm{hr}$, Temp. $=250^{\circ} \mathrm{C}$

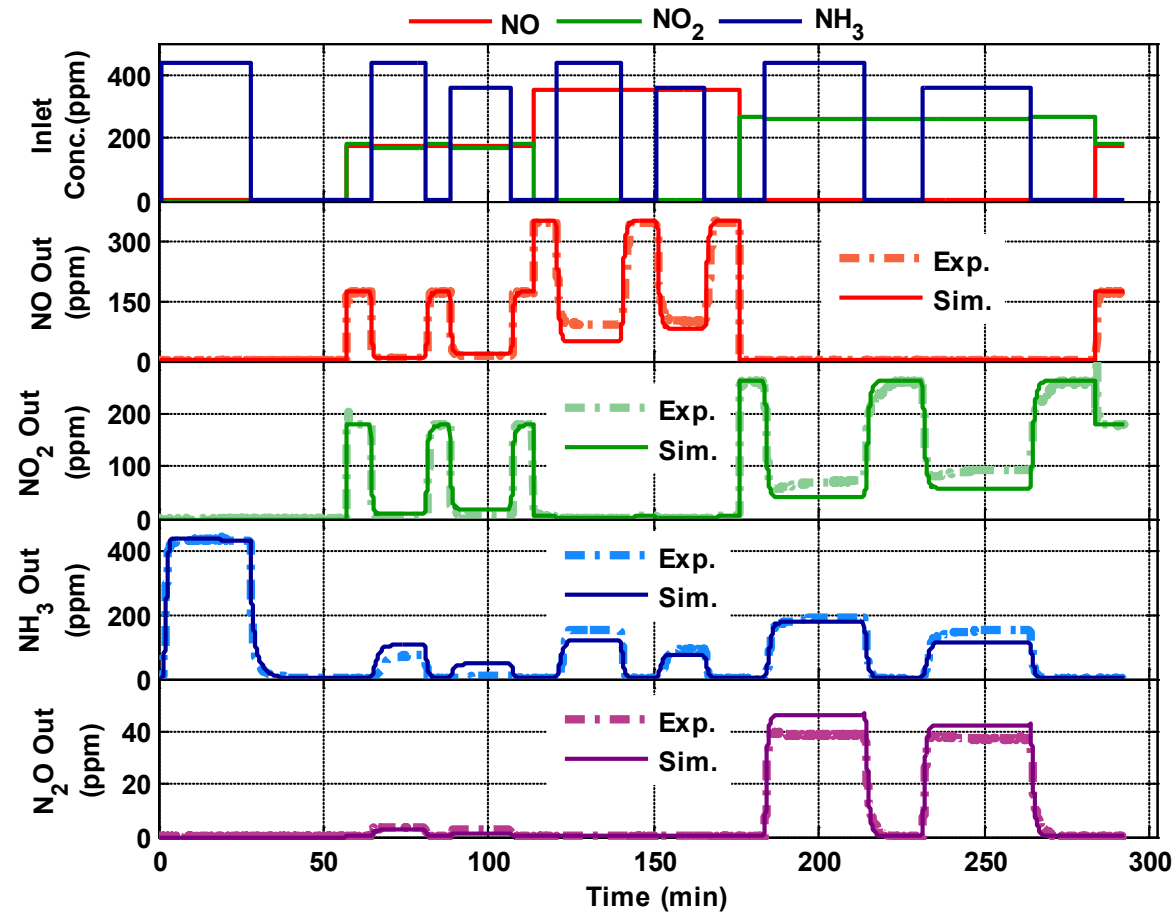

Figure I.18: Model simulation vs. reactor data, SV $=120 \mathrm{k} / \mathrm{hr}, \mathrm{Temp} .=300^{\circ} \mathrm{C}$ 


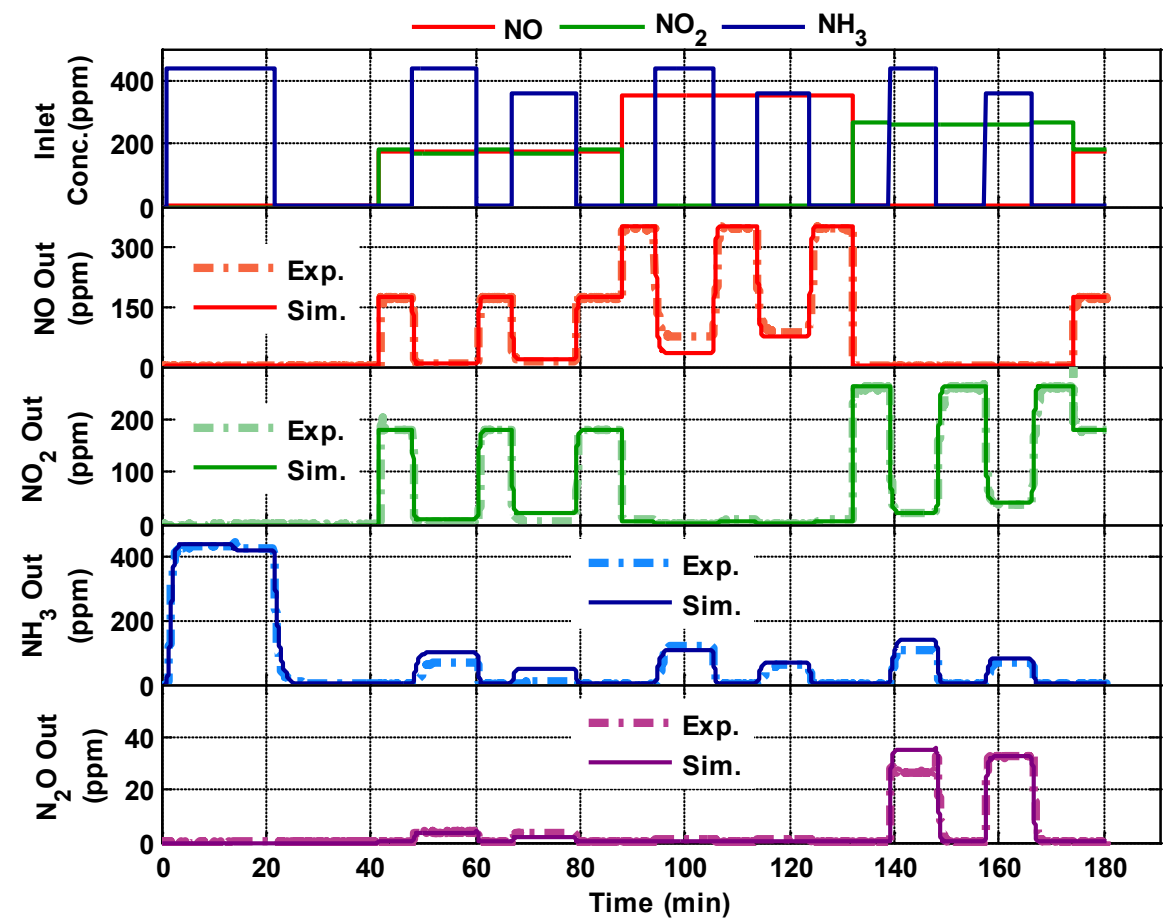

Figure I.19: Model simulation vs. reactor data, SV $=120 \mathrm{k} / \mathrm{hr}$, Temp. $=350^{\circ} \mathrm{C}$

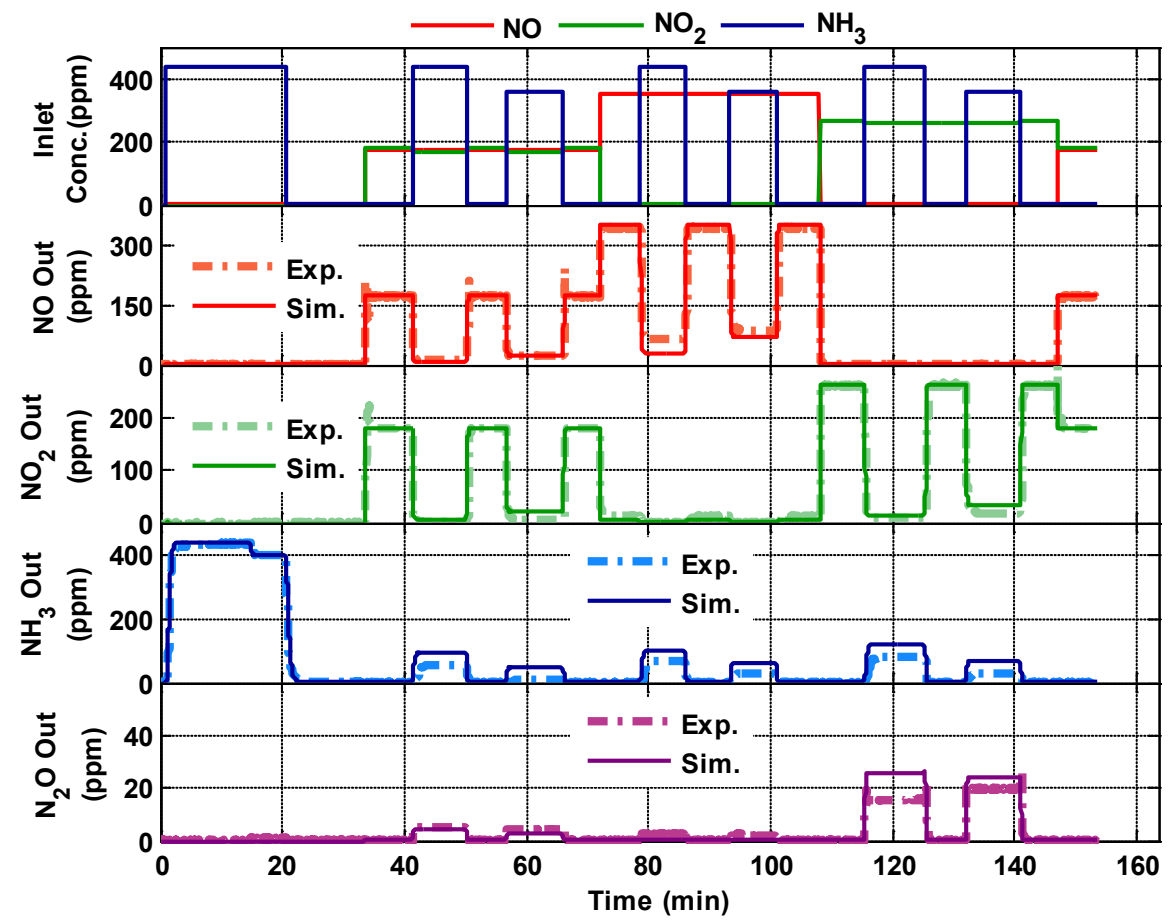

Figure I.20: Model simulation vs. reactor data, SV $=120 \mathrm{k} / \mathrm{hr}$, Temp. $=400^{\circ} \mathrm{C}$ 


\section{Appendix J. Simulations of Steady State}

\section{Engine Experiments}

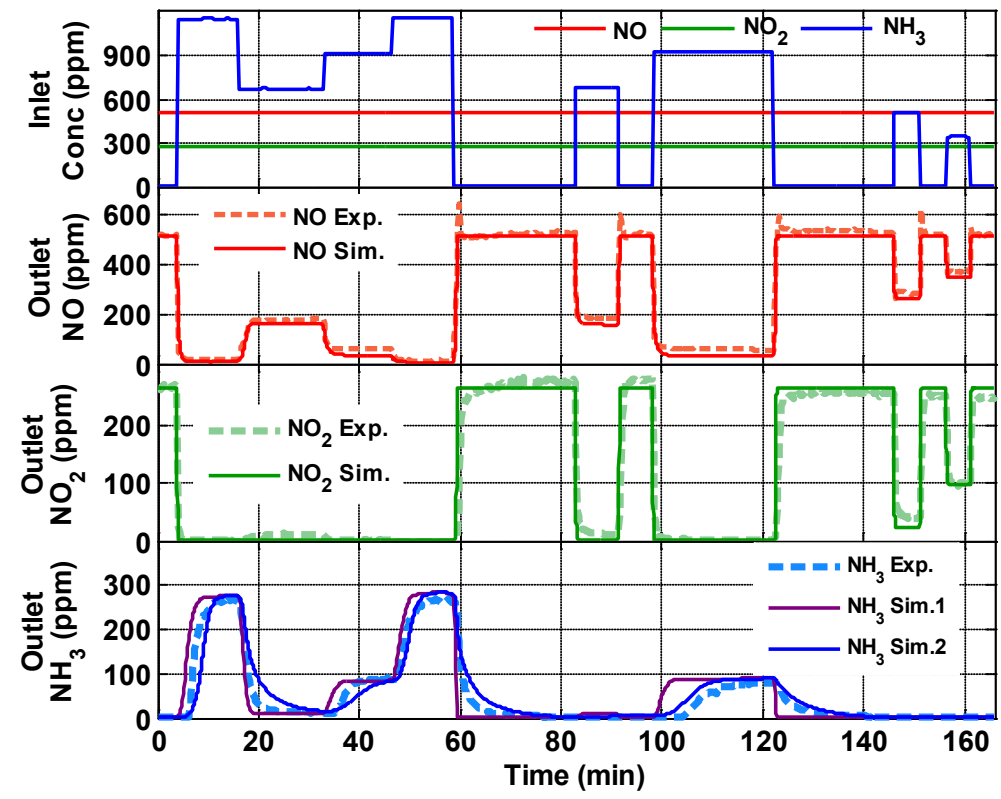

Figure J.1: Model simulation compared to steady state engine data for test point 1 with the SCR inlet temperature of $400^{\circ} \mathrm{C}$

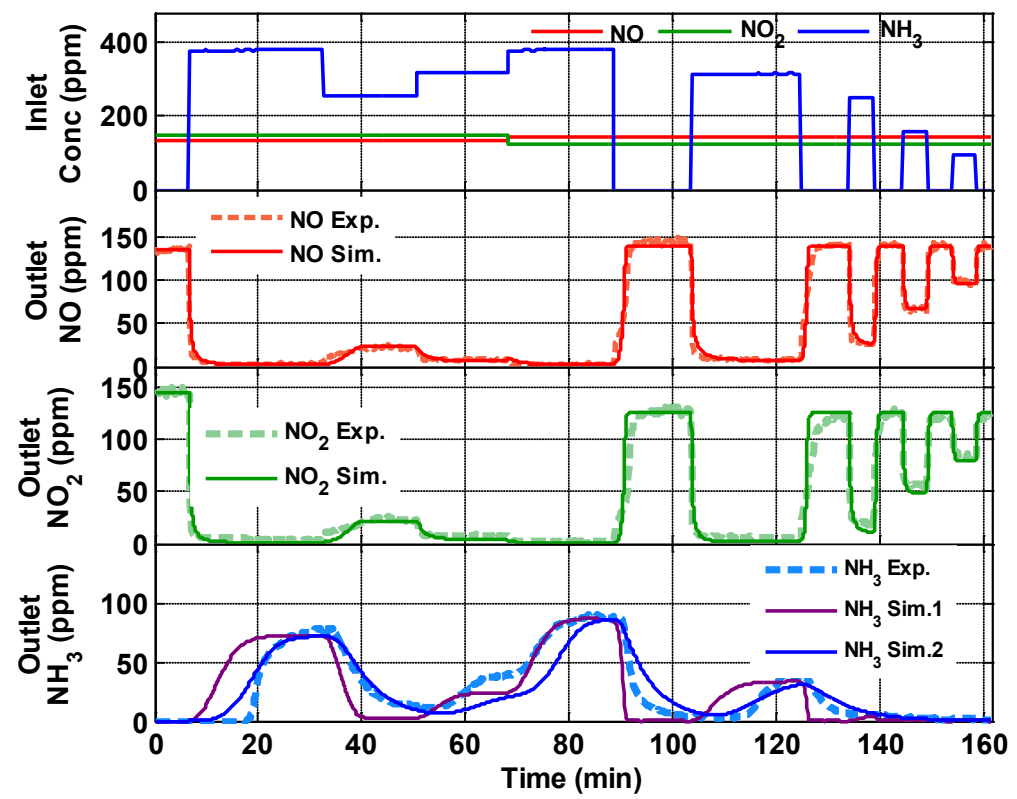

Figure J.2: Model simulation compared to steady state engine data for test point 3 with the SCR inlet temperature of $353^{\circ} \mathrm{C}$ 


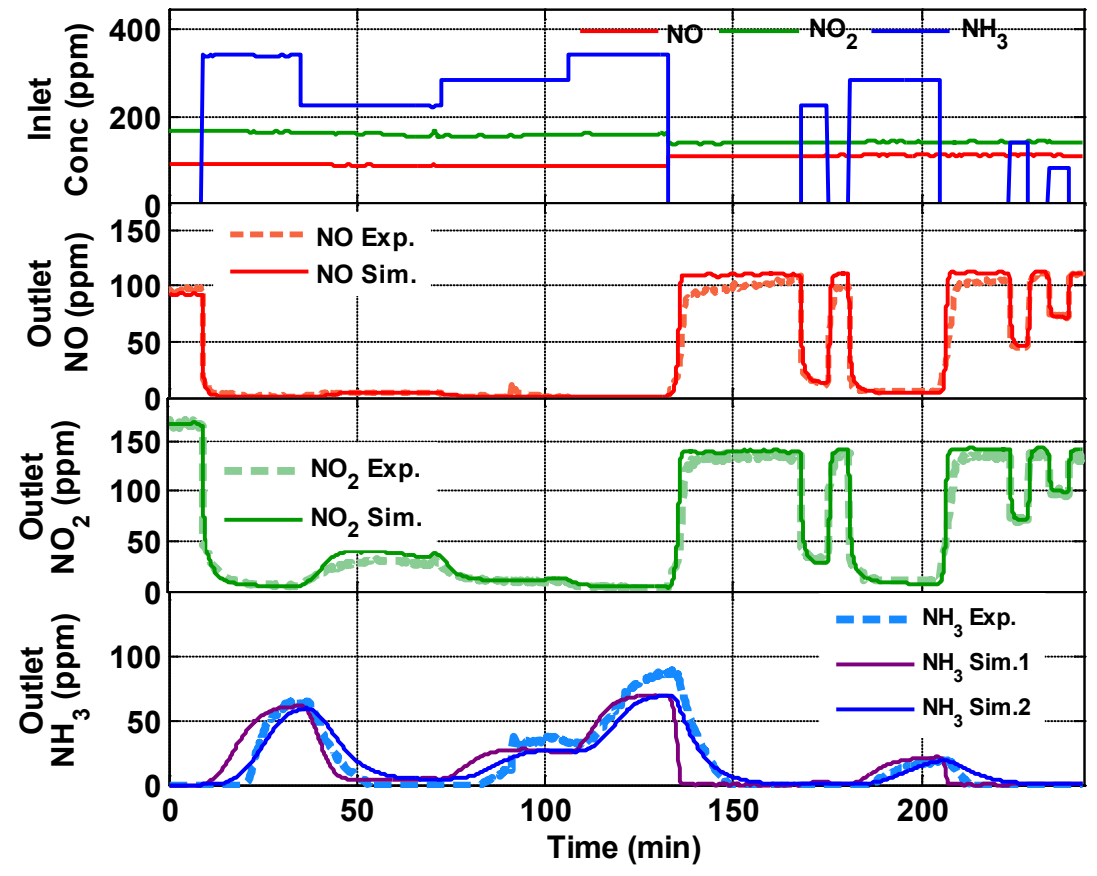

Figure J.3: Model simulation compared to steady state engine data for test point 4 with the SCR inlet temperature of $331^{\circ} \mathrm{C}$

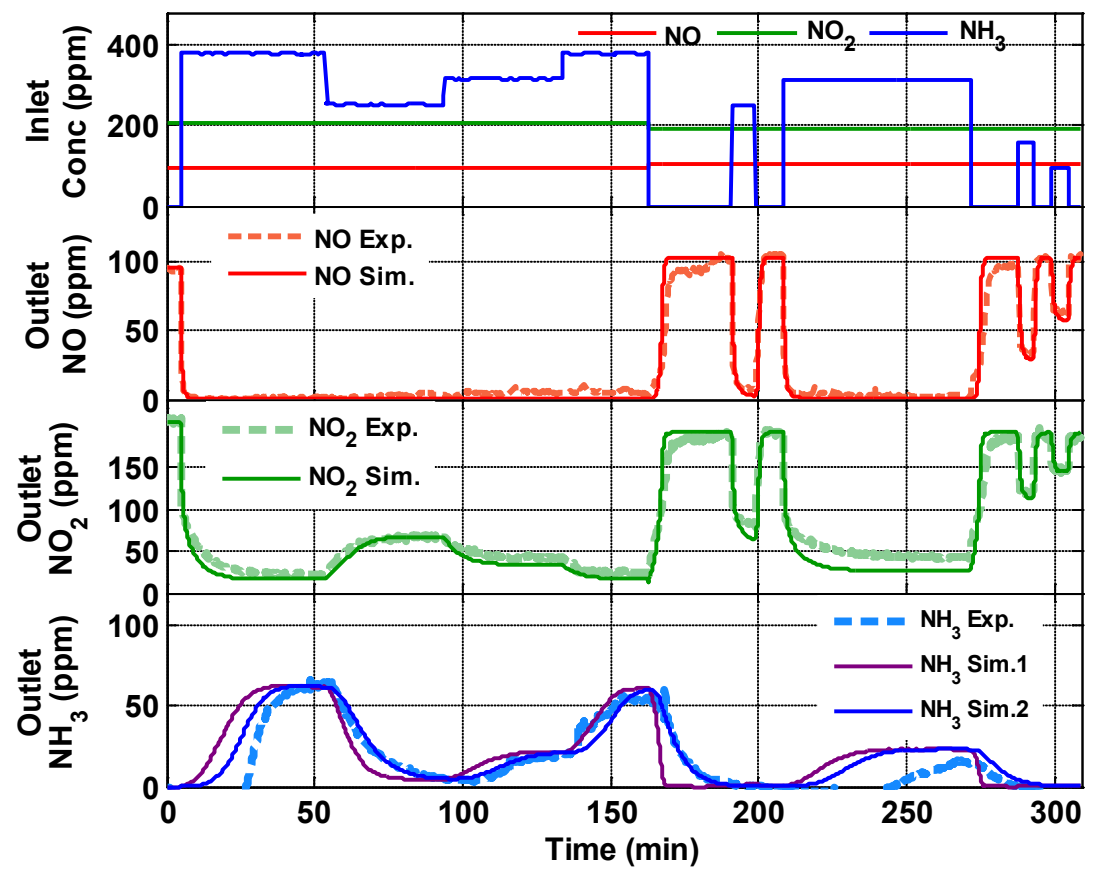

Figure J.4: Model simulation compared to steady state engine data for test point 5 with the SCR inlet temperature of $301^{\circ} \mathrm{C}$ 


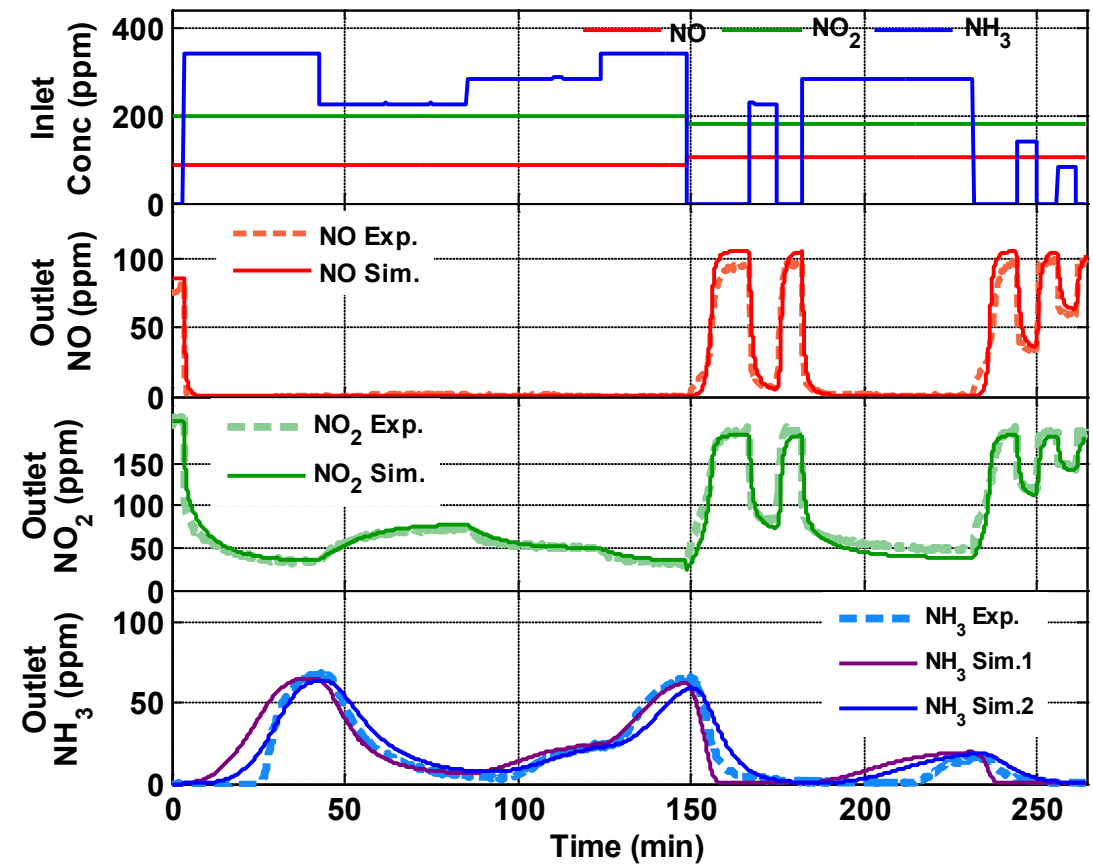

Figure J.5: Model simulation compared to steady state engine data for test point 6 with the SCR inlet temperature of $278^{\circ} \mathrm{C}$

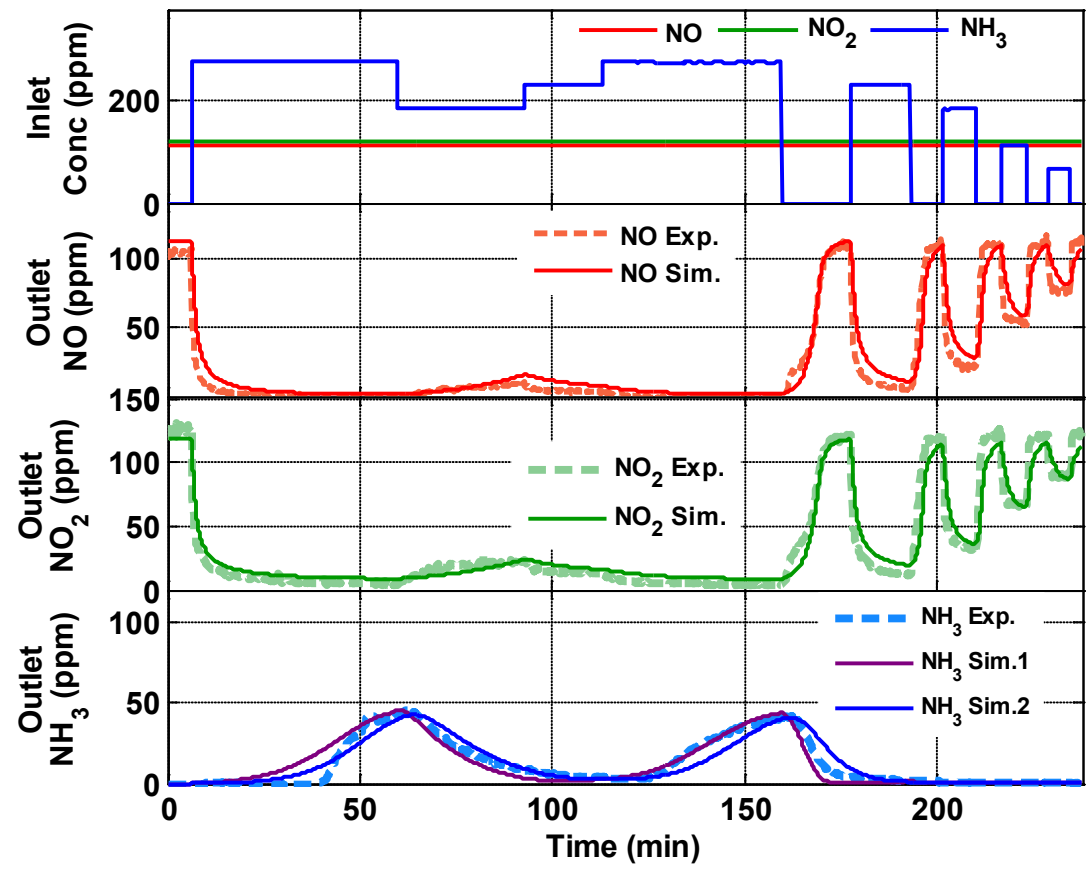

Figure J.6: Model simulation compared to steady state engine data for test point 7 with the SCR inlet temperature of $252^{\circ} \mathrm{C}$ 


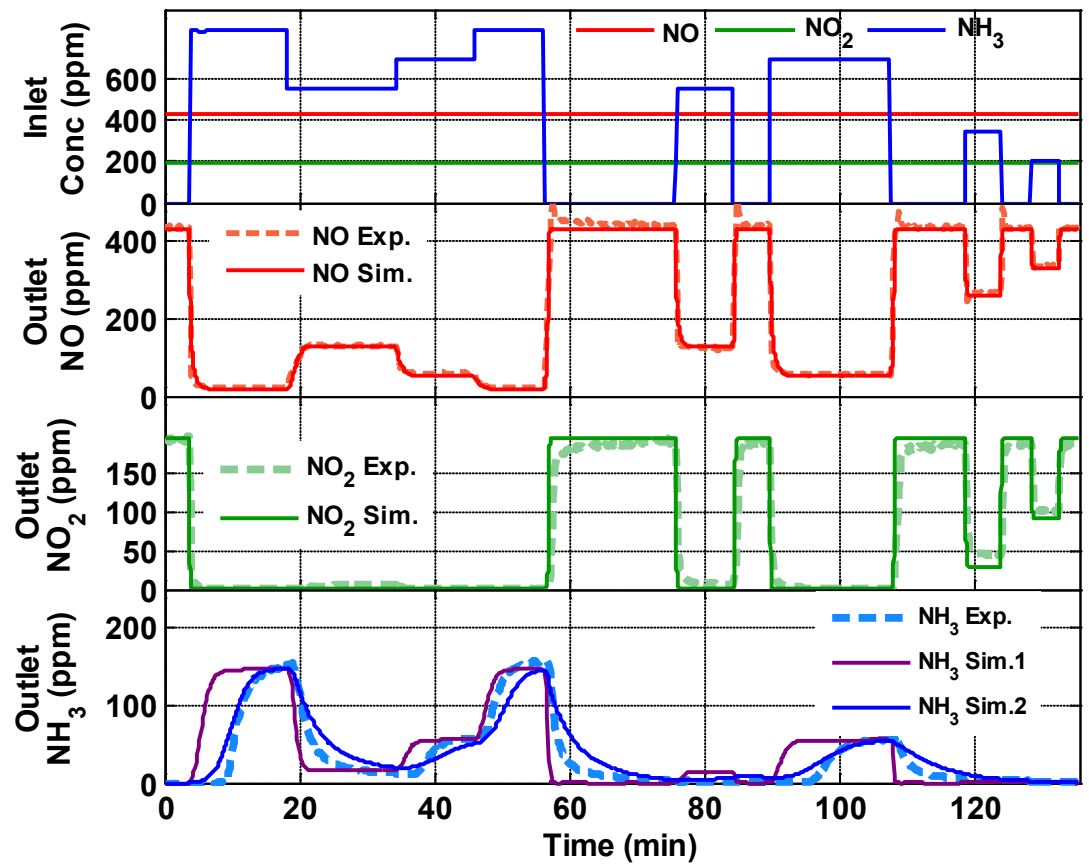

Figure J.7: Model simulation compared to steady state engine data for the repeat of test point 7 with the SCR inlet temperature of $252^{\circ} \mathrm{C}$

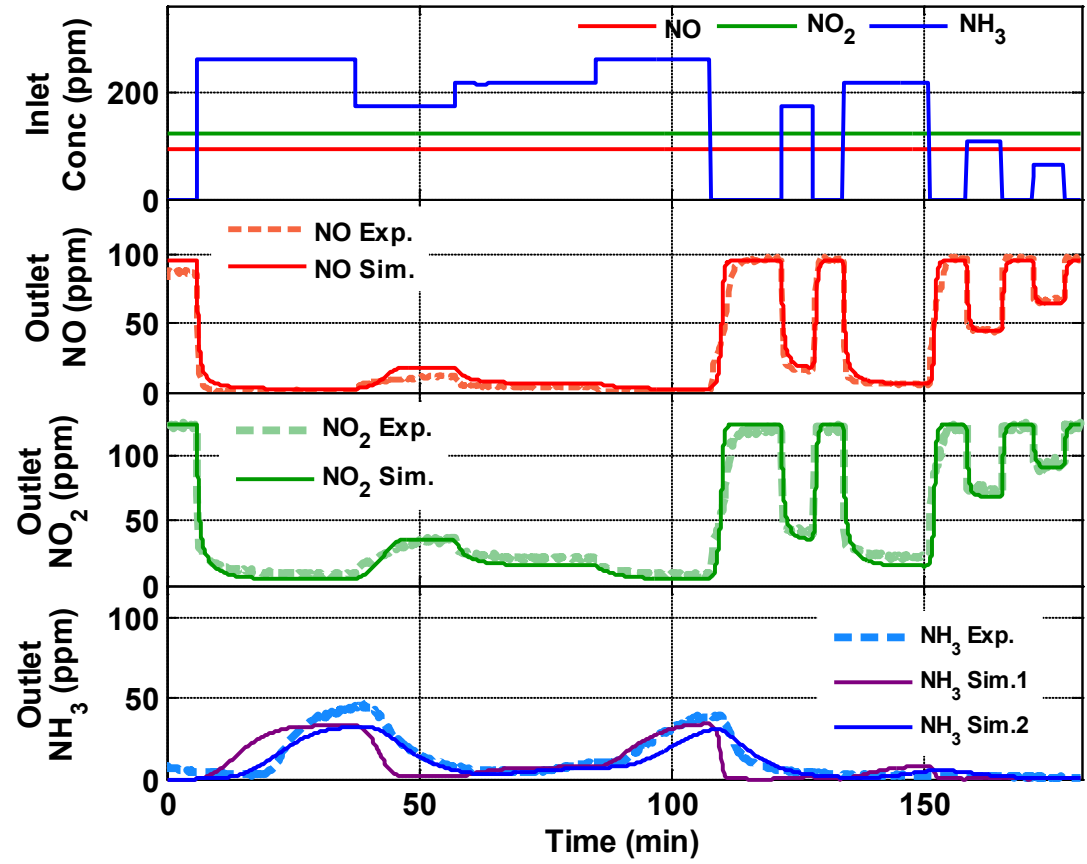

Figure J.8: Model simulation compared to steady state engine data for the repeat of test point 4 with the SCR inlet temperature of $330^{\circ} \mathrm{C}$ 


\section{Appendix K. Simulation Results of Transient SCR Experiments}

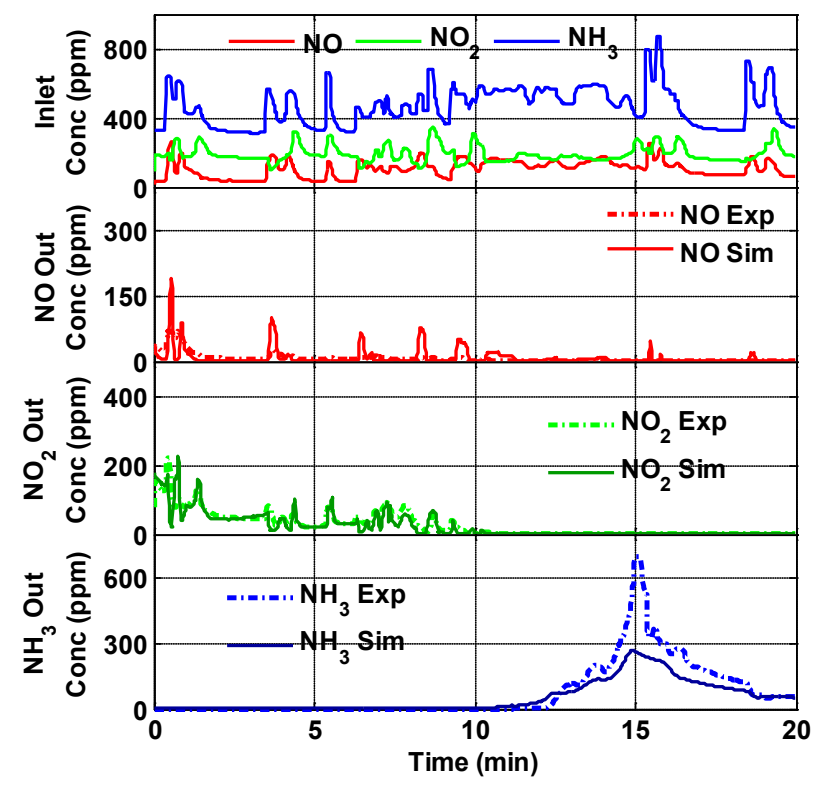

Figure K.1: Simulated concentrations compared to experimental measurements of transient experiment 2

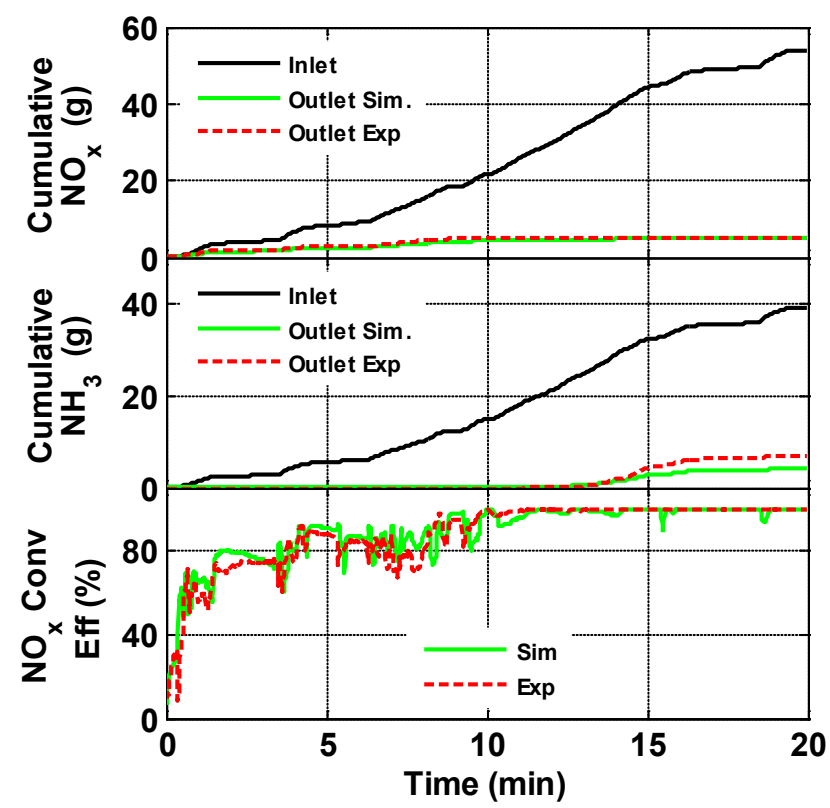

Figure K.2: Simulated cumulative $\mathrm{NO}_{x}, \mathrm{NH}_{3}$ and $\mathrm{NO}_{x}$ conversion efficiency of transient experiment 2 


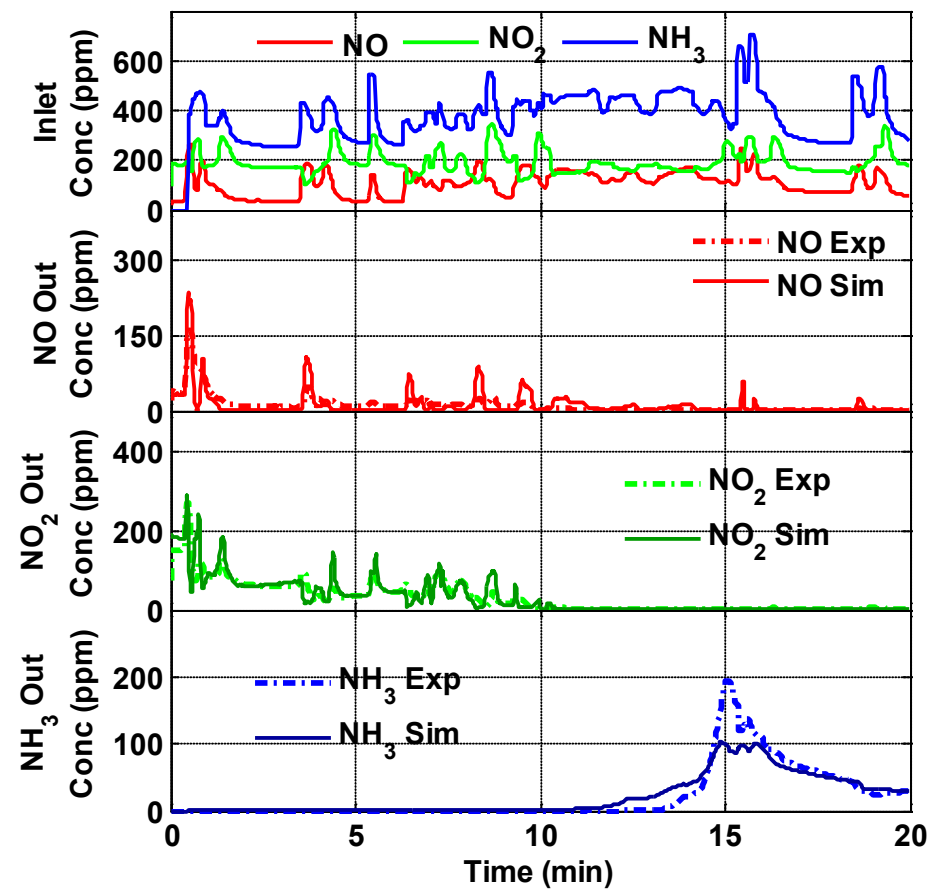

Figure K.3: Simulated concentrations compared to experimental measurements of transient experiment 3

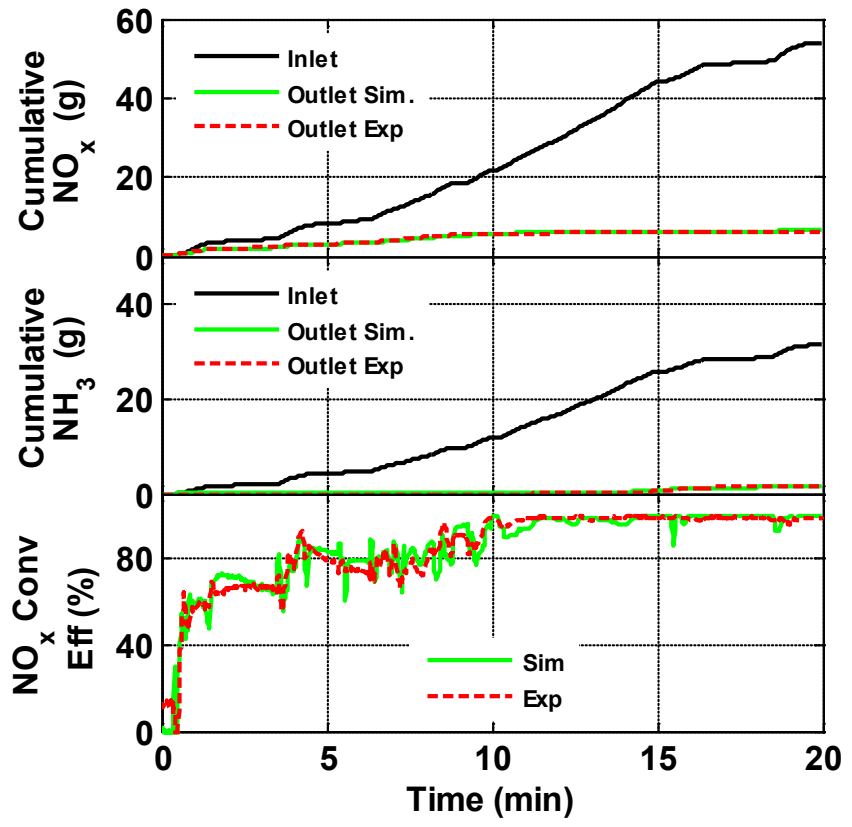

Figure K.4: Simulated cumulative $\mathrm{NO}_{x}, \mathrm{NH}_{3}$ and $\mathrm{NO}_{\mathrm{x}}$ conversion efficiency of transient experiment 3 


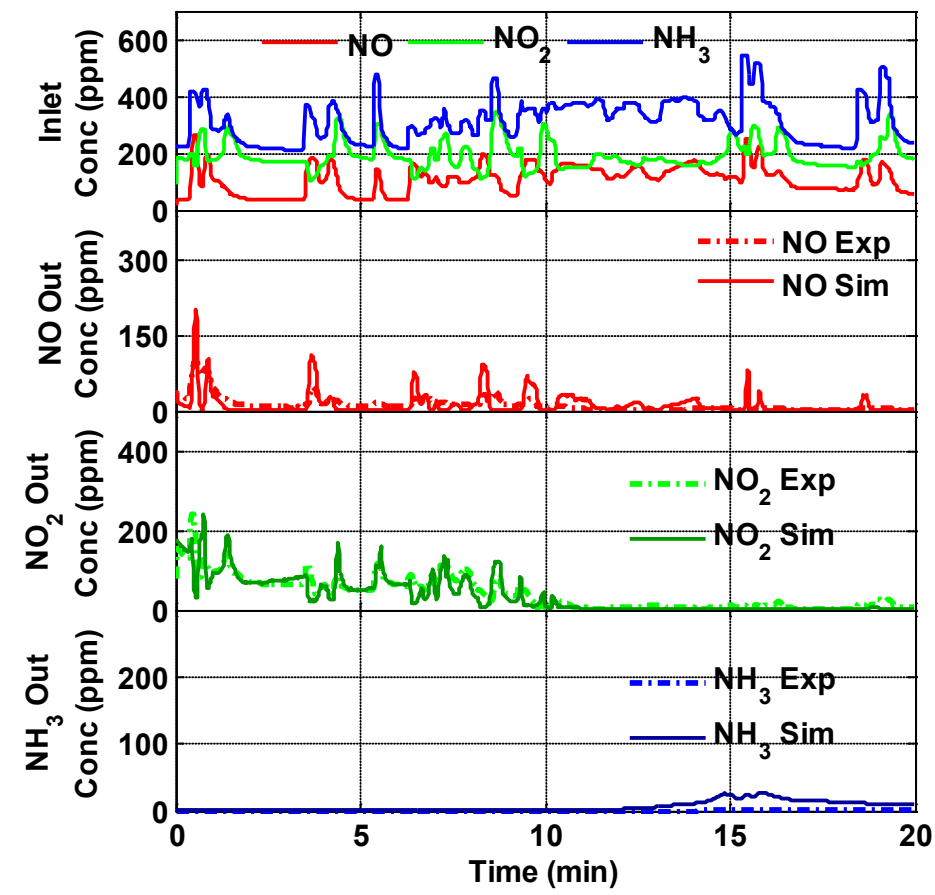

Figure K.5: Simulated concentrations compared to experimental measurements of transient experiment 4

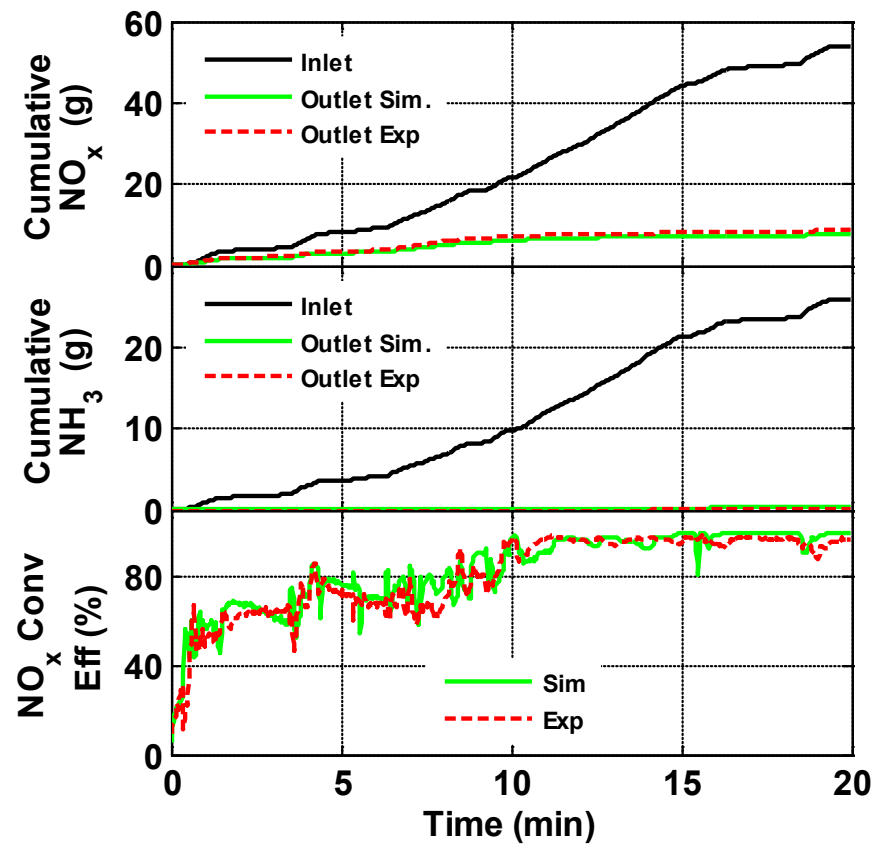

Figure K.6: Simulated cumulative $\mathrm{NO}_{x}, \mathrm{NH}_{3}$ and $\mathrm{NO}_{x}$ conversion efficiency of transient experiment 4 


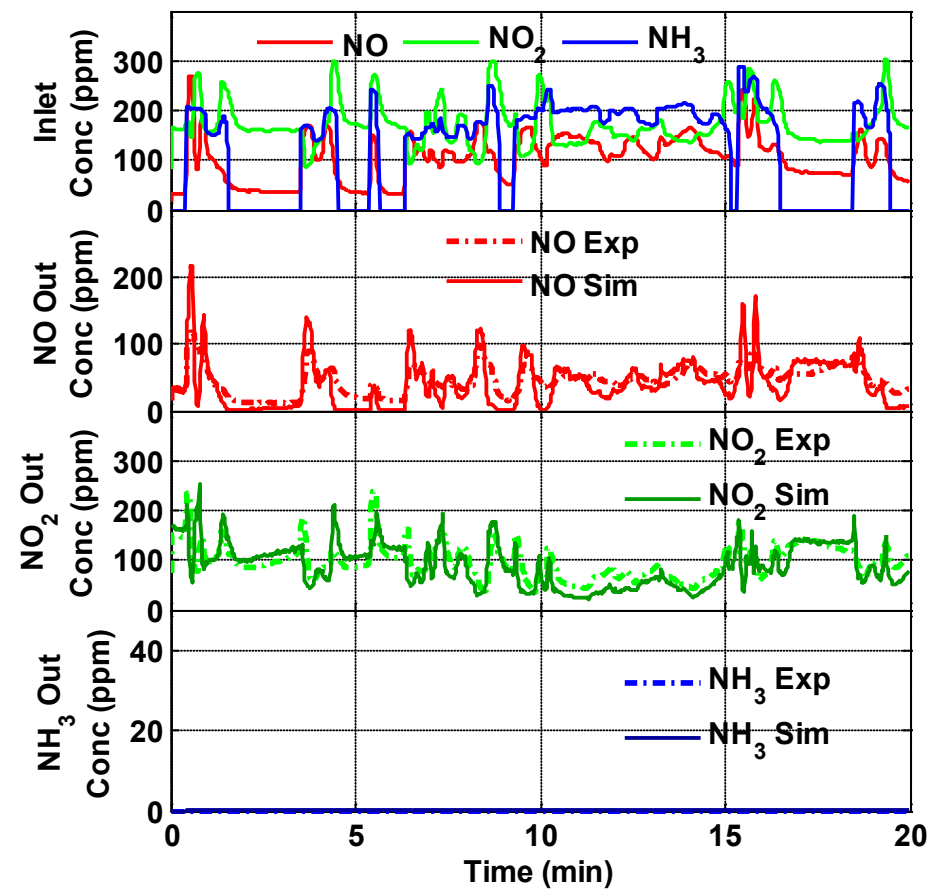

Figure K.7: Simulated concentrations compared to experimental measurements of transient experiment 5

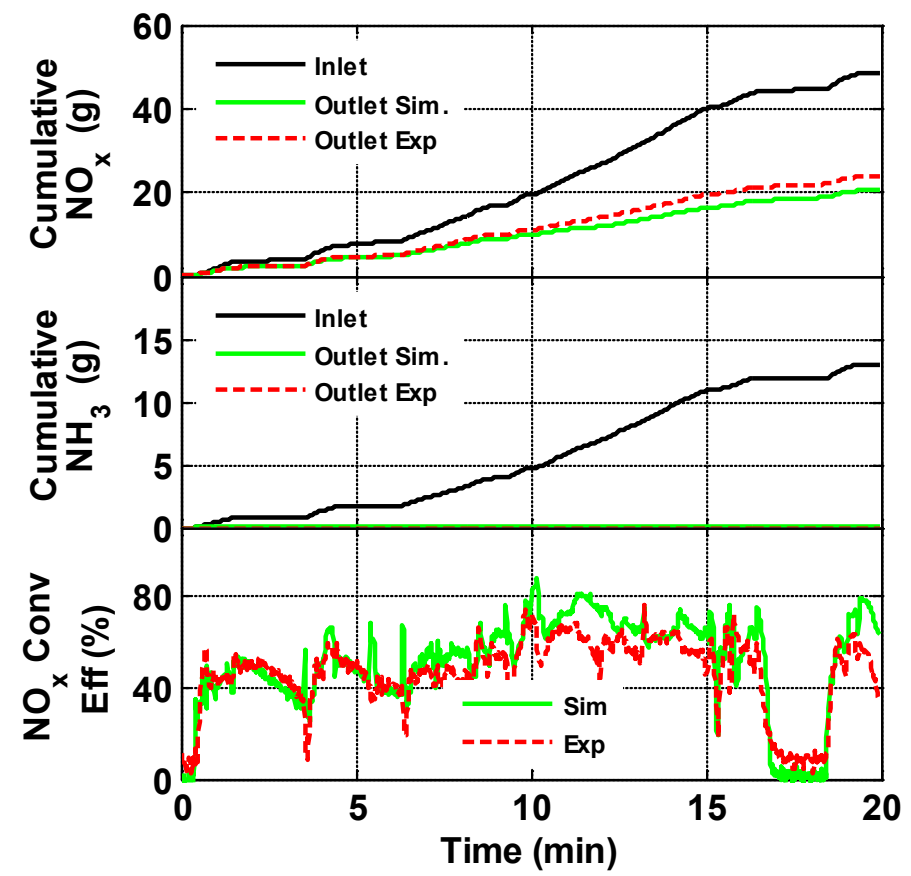

Figure K.8: Simulated cumulative $\mathrm{NO}_{x}, \mathrm{NH}_{3}$ and $\mathrm{NO}_{x}$ conversion efficiency of transient experiment 5 


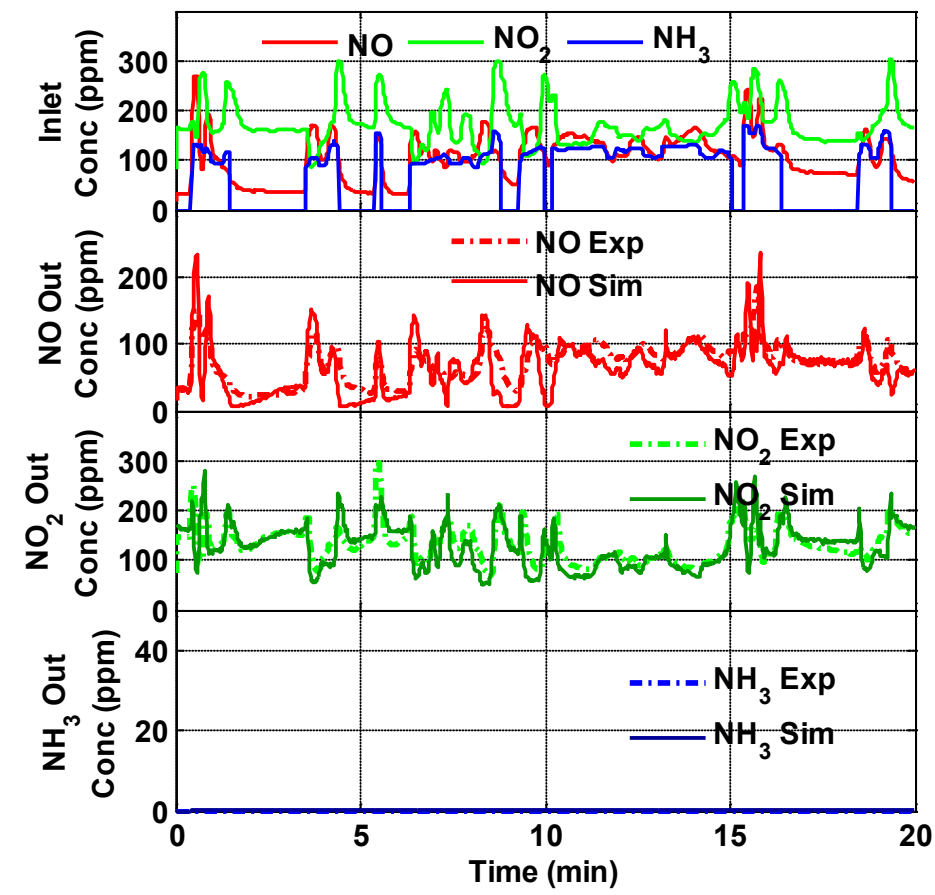

Figure K.9: Simulated concentrations compared to experimental measurements of transient experiment 6

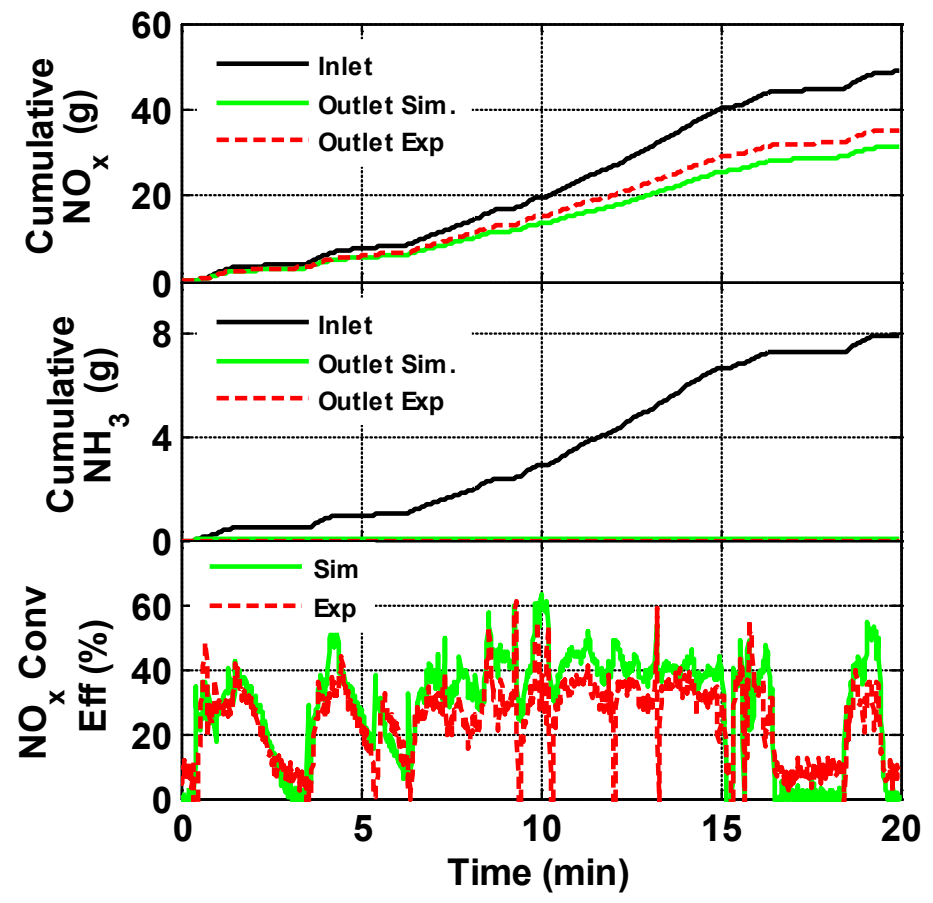

Figure K.10: Simulated cumulative $\mathrm{NO}_{x}, \mathrm{NH}_{3}$ and $\mathrm{NO}_{x}$ conversion efficiency of transient experiment 6 


\section{Appendix L. Results for the $\mathrm{NH}_{3}$ Maldistribution Tests}

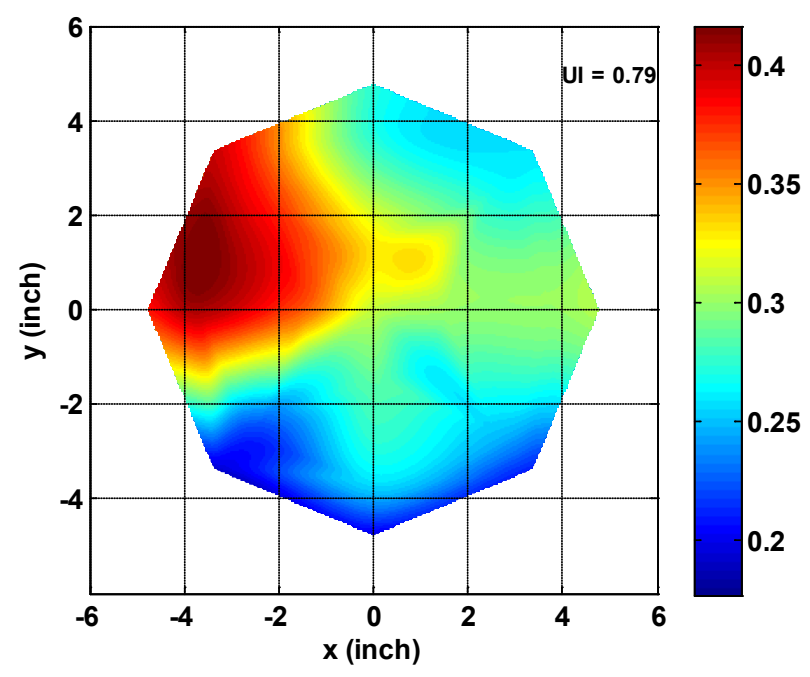

Figure L.1: Distribution profile of the SCR inlet ANR for test point 1

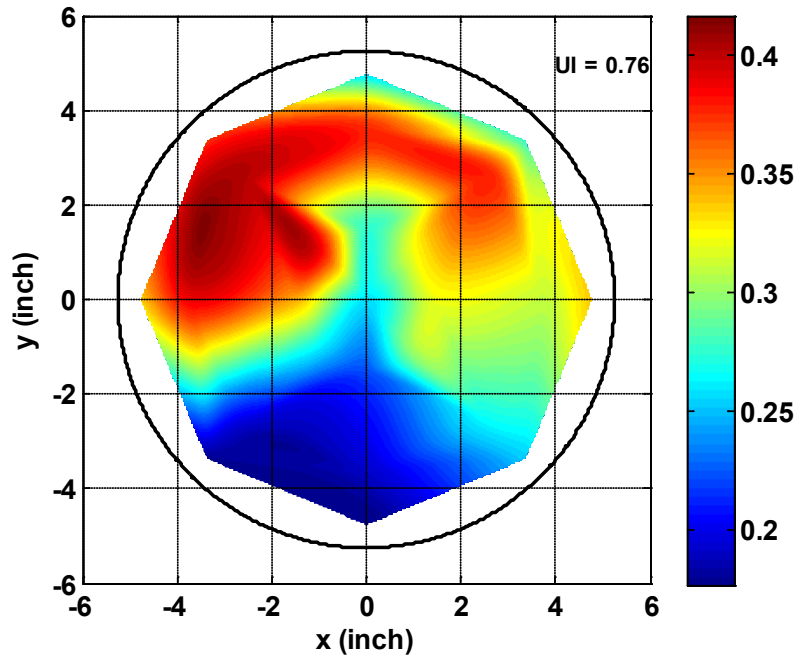

Figure L.2: Distribution profile of the SCR inlet ANR for test point 2 


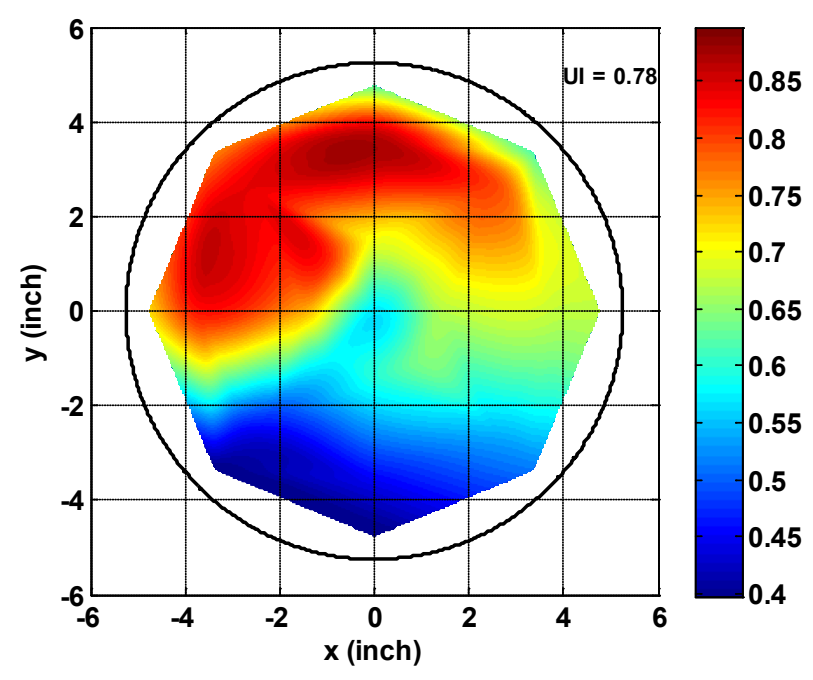

Figure L.3: Distribution profile of the SCR inlet ANR for test point 3

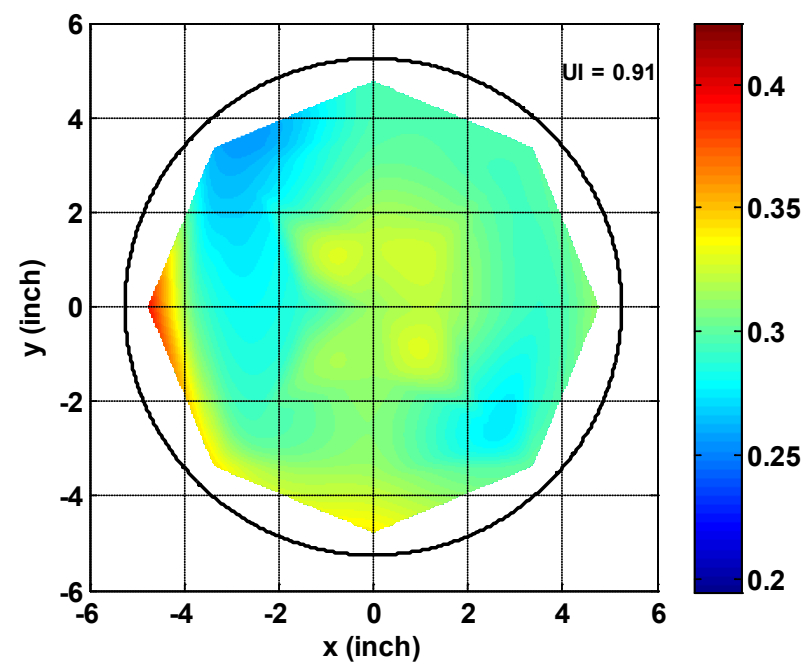

Figure L.4: Distribution profile of the SCR inlet ANR for test point 4 


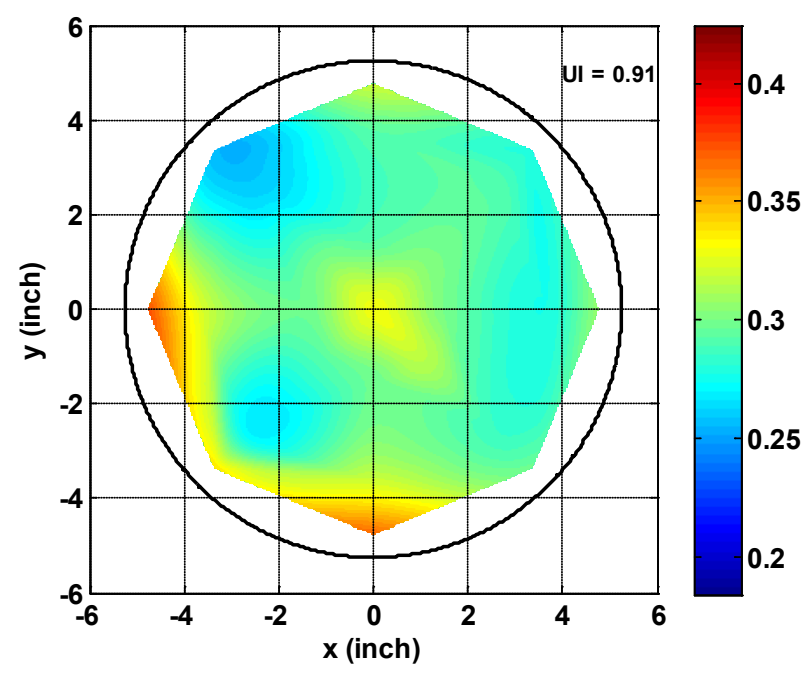

Figure L.5: Distribution profile of the SCR inlet ANR for test point 5

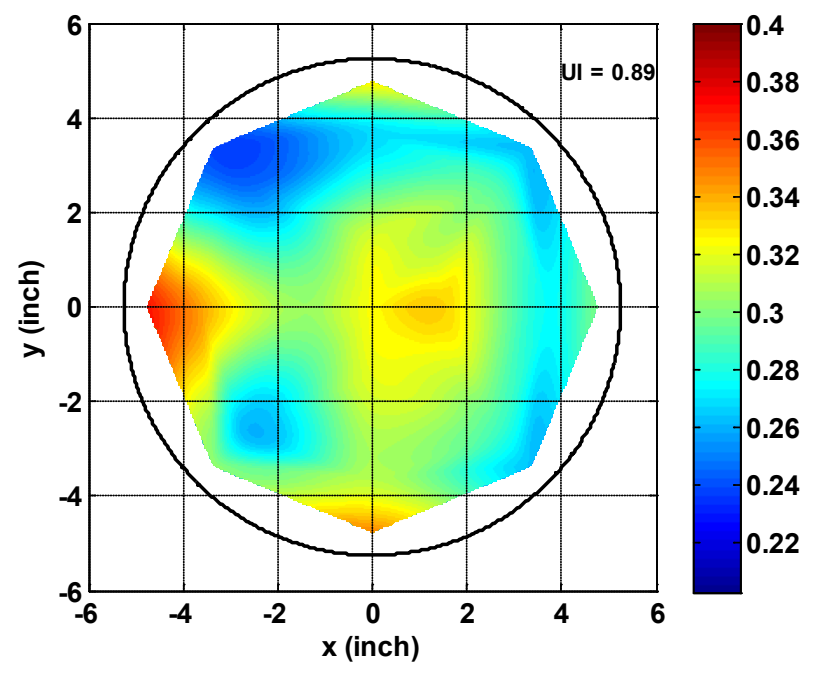

Figure L.6: Distribution profile of the SCR inlet ANR for test point 6 


\section{Appendix M. Permission to Use Copyrighted Material}

\section{Permission to Use Figure 2.1}

\section{ELSEVIER LICENSE TERMS AND CONDITIONS}

Jul 05, 2013

This is a License Agreement between Xiaobo Song ("You") and Elsevier ("Elsevier") provided by Copyright Clearance Center ("CCC"). The license consists of your order details, the terms and conditions provided by Elsevier, and the payment terms and conditions.

\begin{tabular}{|c|c|}
\hline \multicolumn{2}{|c|}{$\begin{array}{l}\text { All payments must be made in full to CCC. For payment instructions, please see } \\
\text { information listed at the bottom of this form. }\end{array}$} \\
\hline Supplier & $\begin{array}{l}\text { Elsevier Limited } \\
\text { The Boulevard,Langford Lane } \\
\text { Kidlington,Oxford,OX5 1GB,UK }\end{array}$ \\
\hline Registered Company Number & 1982084 \\
\hline Customer name & Xiaobo Song \\
\hline \multirow[t]{2}{*}{ Customer address } & 1400 Townsend $\mathrm{Dr}$ \\
\hline & HOUGHTON, MI 49931 \\
\hline License number & 3182661168532 \\
\hline License date & Jul 05, 2013 \\
\hline Licensed content publisher & Elsevier \\
\hline Licensed content publication & Catalysis Today \\
\hline Licensed content title & $\begin{array}{l}\text { Mechanistic study of the low temperature activity of } \\
\text { transition metal exchanged zeolite SCR catalysts }\end{array}$ \\
\hline Licensed content author & Joseph M. Fedeyko,Bin Chen,Hai-Ying Chen \\
\hline Licensed content date & 19 June 2010 \\
\hline Licensed content volume number & 151 \\
\hline Licensed content issue number & $3-4$ \\
\hline Number of pages & 6 \\
\hline Start Page & 231 \\
\hline
\end{tabular}




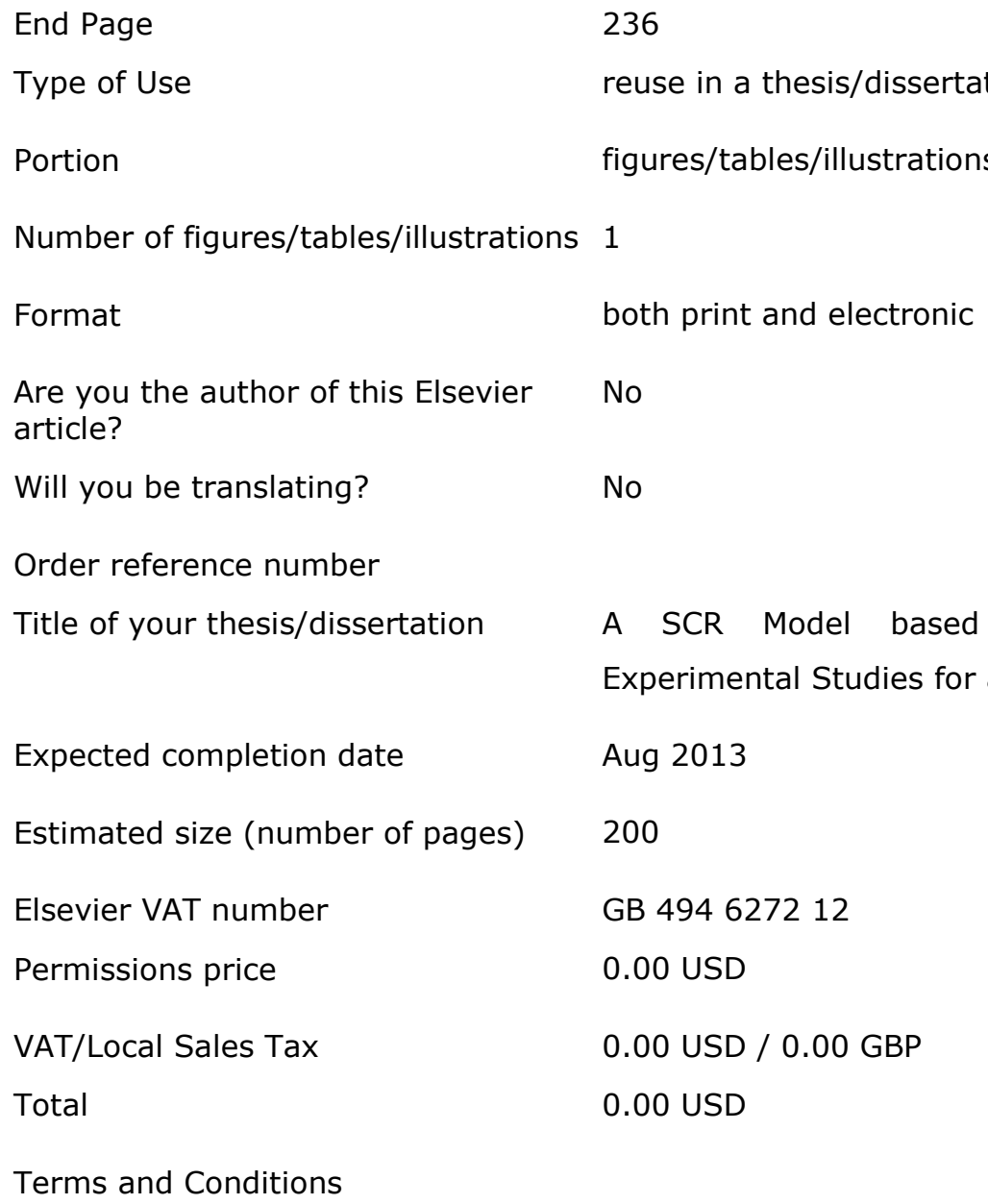

Terms and Conditions

\section{INTRODUCTION}

1. The publisher for this copyrighted material is Elsevier. By clicking "accept" in connection with completing this licensing transaction, you agree that the following terms and conditions apply to this transaction (along with the Billing and Payment terms and conditions established by Copyright Clearance Center, Inc. ("CCC"), at the time that you opened your Rightslink account and that are available at any time at http://myaccount.copyright.com).

\section{GENERAL TERMS}

2. Elsevier hereby grants you permission to reproduce the aforementioned material subject to the terms and conditions indicated.

3. Acknowledgement: If any part of the material to be used (for example, figures) has appeared in our publication with credit or acknowledgement to another source, 
permission must also be sought from that source. If such permission is not obtained then that material may not be included in your publication/copies. Suitable acknowledgement to the source must be made, either as a footnote or in a reference list at the end of your publication, as follows:

"Reprinted from Publication title, Vol /edition number, Author(s), Title of article / title of chapter, Pages No., Copyright (Year), with permission from Elsevier [OR APPLICABLE SOCIETY COPYRIGHT OWNER]." Also Lancet special credit "Reprinted from The Lancet, Vol. number, Author(s), Title of article, Pages No., Copyright (Year), with permission from Elsevier."

4. Reproduction of this material is confined to the purpose and/or media for which permission is hereby given.

5. Altering/Modifying Material: Not Permitted. However figures and illustrations may be altered/adapted minimally to serve your work. Any other abbreviations, additions, deletions and/or any other alterations shall be made only with prior written authorization of Elsevier Ltd. (Please contact Elsevier at permissions@elsevier.com)

6. If the permission fee for the requested use of our material is waived in this instance, please be advised that your future requests for Elsevier materials may attract a fee.

7. Reservation of Rights: Publisher reserves all rights not specifically granted in the combination of (i) the license details provided by you and accepted in the course of this licensing transaction, (ii) these terms and conditions and (iii) CCC's Billing and Payment terms and conditions.

8. License Contingent Upon Payment: While you may exercise the rights licensed immediately upon issuance of the license at the end of the licensing process for the transaction, provided that you have disclosed complete and accurate details of your proposed use, no license is finally effective unless and until full payment is received from you (either by publisher or by CCC) as provided in CCC's Billing and Payment terms and conditions. If full payment is not received on a timely basis, then any license preliminarily granted shall be deemed automatically revoked and shall be void as if never granted. Further, in the event that you breach any of these terms and conditions or any of CCC's Billing and Payment terms and conditions, the license is automatically revoked and shall be void as if never granted. Use of materials as described in a revoked license, as well as any use of the materials beyond the scope of an unrevoked license, may constitute copyright infringement and publisher reserves the right to take any and all action to protect its copyright in the materials.

9. Warranties: Publisher makes no representations or warranties with respect to the 
licensed material.

10. Indemnity: You hereby indemnify and agree to hold harmless publisher and CCC, and their respective officers, directors, employees and agents, from and against any and all claims arising out of your use of the licensed material other than as specifically authorized pursuant to this license.

11. No Transfer of License: This license is personal to you and may not be sublicensed, assigned, or transferred by you to any other person without publisher's written permission.

12. No Amendment Except in Writing: This license may not be amended except in a writing signed by both parties (or, in the case of publisher, by CCC on publisher's behalf).

13. Objection to Contrary Terms: Publisher hereby objects to any terms contained in any purchase order, acknowledgment, check endorsement or other writing prepared by you, which terms are inconsistent with these terms and conditions or CCC's Billing and Payment terms and conditions. These terms and conditions, together with CCC's Billing and Payment terms and conditions (which are incorporated herein), comprise the entire agreement between you and publisher (and CCC) concerning this licensing transaction. In the event of any conflict between your obligations established by these terms and conditions and those established by $\mathrm{CCC}^{\prime}$ s Billing and Payment terms and conditions, these terms and conditions shall control.

14. Revocation: Elsevier or Copyright Clearance Center may deny the permissions described in this License at their sole discretion, for any reason or no reason, with a full refund payable to you. Notice of such denial will be made using the contact information provided by you. Failure to receive such notice will not alter or invalidate the denial. In no event will Elsevier or Copyright Clearance Center be responsible or liable for any costs, expenses or damage incurred by you as a result of a denial of your permission request, other than a refund of the amount(s) paid by you to Elsevier and/or Copyright Clearance Center for denied permissions.

\section{LIMITED LICENSE}

The following terms and conditions apply only to specific license types:

15. Translation: This permission is granted for non-exclusive world English rights only unless your license was granted for translation rights. If you licensed translation rights you may only translate this content into the languages you requested. A professional translator must perform all translations and reproduce the content word for word preserving the integrity of the article. If this license is to re-use 1 or 2 figures then 
permission is granted for non-exclusive world rights in all languages.

16. Website: The following terms and conditions apply to electronic reserve and author websites:

Electronic reserve: If licensed material is to be posted to website, the web site is to be password-protected and made available only to bona fide students registered on a relevant course

if: This license was made in connection with a course, This permission is granted for 1 year only. You may obtain a license for future website posting,

All content posted to the web site must maintain the copyright information line on the bottom of each image, A hyper-text must be included to the Homepage of the journal from which you are licensing athttp://www.sciencedirect.com/science/journal/xxxxx or the Elsevier homepage for books athttp://www.elsevier.com, and Central Storage: This license does not include permission for a scanned version of the material to be stored in a central repository such as that provided by Heron/XanEdu.

17. Author website for journals with the following additional clauses:

All content posted to the web site must maintain the copyright information line on the bottom of each image, and the permission granted is limited to the personal version of your paper. You are not allowed to download and post the published electronic version of your article (whether PDF or HTML, proof or final version), nor may you scan the printed edition to create an electronic version. A hyper-text must be included to the Homepage of the journal from which you are licensing athttp://www.sciencedirect.com/science/journal/xxxxx . As part of our normal production process, you will receive an e-mail notice when your article appears on Elsevier's online service ScienceDirect (www.sciencedirect.com). That e-mail will include the article's Digital Object Identifier (DOI). This number provides the electronic link to the published article and should be included in the posting of your personal version. We ask that you wait until you receive this e-mail and have the DOI to do any posting.

Central Storage: This license does not include permission for a scanned version of the material to be stored in a central repository such as that provided by Heron/XanEdu.

18. Author website for books with the following additional clauses: Authors are permitted to place a brief summary of their work online only. A hyper-text must be included to the Elsevier homepage at http://www.elsevier.com . All content posted to the web site must maintain the copyright information line on the bottom of each image. You are not allowed to download and post the published electronic version of your chapter, nor may you scan the printed edition to create an 
electronic version.

Central Storage: This license does not include permission for a scanned version of the material to be stored in a central repository such as that provided by Heron/XanEdu.

19. Website (regular and for author): A hyper-text must be included to the Homepage of the journal from which you are licensing at http://www.sciencedirect.com/science/journal/xxxxx. or for books to the Elsevier homepage at http://www.elsevier.com

20. Thesis/Dissertation: If your license is for use in a thesis/dissertation your thesis may be submitted to your institution in either print or electronic form. Should your thesis be published commercially, please reapply for permission. These requirements include permission for the Library and Archives of Canada to supply single copies, on demand, of the complete thesis and include permission for UMI to supply single copies, on demand, of the complete thesis. Should your thesis be published commercially, please reapply for permission.

\section{Other Conditions:}

v1.6

If you would like to pay for this license now, please remit this license along with your payment made payable to "COPYRIGHT CLEARANCE CENTER" otherwise you will be invoiced within $\mathbf{4 8}$ hours of the license date. Payment should be in the form of a check or money order referencing your account number and this invoice number RLNK501059544.

Once you receive your invoice for this order, you may pay your invoice by credit card. Please follow instructions provided at that time.

Make Payment To:

Copyright Clearance Center

Dept 001

P.0. Box 843006

Boston, MA 02284-3006

For suggestions or comments regarding this order, contact RightsLink Customer Support:customercare@copyright.com or +1-877-622-5543 (toll free in the US) or +1-978-646-2777.

Gratis licenses (referencing $\$ 0$ in the Total field) are free. Please retain this printable license for your reference. No payment is required. 


\section{Permissions to Use Figure 2.2}

\section{ELSEVIER LICENSE TERMS AND CONDITIONS}

Jul 05, 2013

This is a License Agreement between Xiaobo Song ("You") and Elsevier ("Elsevier") provided by Copyright Clearance Center ("CCC"). The license consists of your order details, the terms and conditions provided by Elsevier, and the payment terms and conditions.

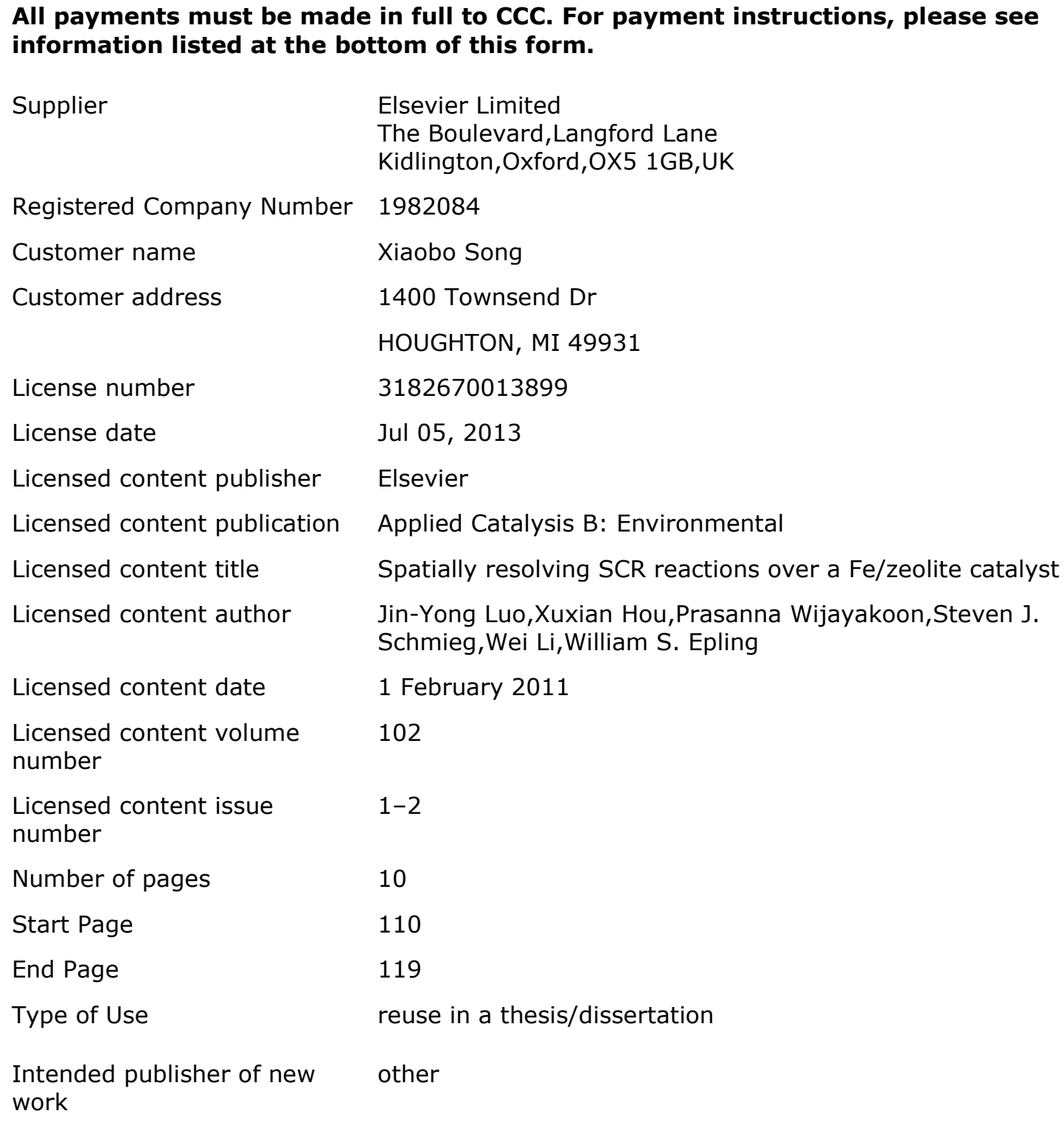




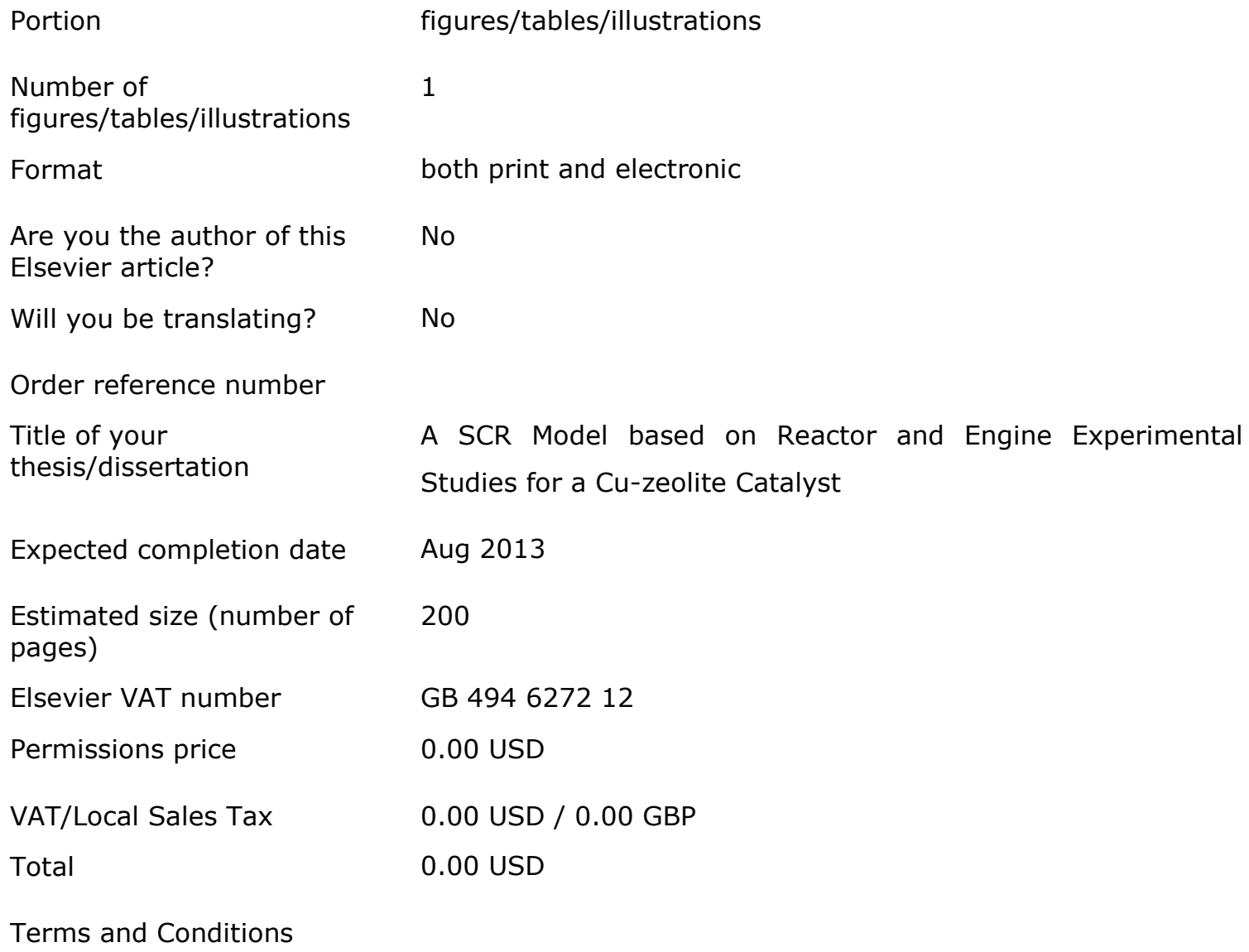

\section{INTRODUCTION}

1. The publisher for this copyrighted material is Elsevier. By clicking "accept" in connection with completing this licensing transaction, you agree that the following terms and conditions apply to this transaction (along with the Billing and Payment terms and conditions established by Copyright Clearance Center, Inc. ("CCC"), at the time that you opened your Rightslink account and that are available at any time at http://myaccount.copyright.com).

\section{GENERAL TERMS}

2. Elsevier hereby grants you permission to reproduce the aforementioned material subject to the terms and conditions indicated.

3. Acknowledgement: If any part of the material to be used (for example, figures) has appeared in our publication with credit or acknowledgement to another source, permission must also be sought from that source. If such permission is not obtained then that material may not be included in your publication/copies. Suitable acknowledgement to the source must be made, either as a footnote or in a reference list at the end of your 
publication, as follows:

"Reprinted from Publication title, Vol /edition number, Author(s), Title of article / title of chapter, Pages No., Copyright (Year), with permission from Elsevier [OR APPLICABLE SOCIETY COPYRIGHT OWNER]." Also Lancet special credit "Reprinted from The Lancet, Vol. number, Author(s), Title of article, Pages No., Copyright (Year), with permission from Elsevier."

4. Reproduction of this material is confined to the purpose and/or media for which permission is hereby given.

5. Altering/Modifying Material: Not Permitted. However figures and illustrations may be altered/adapted minimally to serve your work. Any other abbreviations, additions, deletions and/or any other alterations shall be made only with prior written authorization of Elsevier Ltd. (Please contact Elsevier at permissions@elsevier.com)

6. If the permission fee for the requested use of our material is waived in this instance, please be advised that your future requests for Elsevier materials may attract a fee.

7. Reservation of Rights: Publisher reserves all rights not specifically granted in the combination of (i) the license details provided by you and accepted in the course of this licensing transaction, (ii) these terms and conditions and (iii) CCC's Billing and Payment terms and conditions.

8. License Contingent Upon Payment: While you may exercise the rights licensed immediately upon issuance of the license at the end of the licensing process for the transaction, provided that you have disclosed complete and accurate details of your proposed use, no license is finally effective unless and until full payment is received from you (either by publisher or by CCC) as provided in CCC's Billing and Payment terms and conditions. If full payment is not received on a timely basis, then any license preliminarily granted shall be deemed automatically revoked and shall be void as if never granted. Further, in the event that you breach any of these terms and conditions or any of CCC's Billing and Payment terms and conditions, the license is automatically revoked and shall be void as if never granted. Use of materials as described in a revoked license, as well as any use of the materials beyond the scope of an unrevoked license, may constitute copyright infringement and publisher reserves the right to take any and all action to protect its copyright in the materials.

9. Warranties: Publisher makes no representations or warranties with respect to the licensed material.

10. Indemnity: You hereby indemnify and agree to hold harmless publisher and CCC, and their respective officers, directors, employees and agents, from and against any and 
all claims arising out of your use of the licensed material other than as specifically authorized pursuant to this license.

11. No Transfer of License: This license is personal to you and may not be sublicensed, assigned, or transferred by you to any other person without publisher's written permission.

12. No Amendment Except in Writing: This license may not be amended except in a writing signed by both parties (or, in the case of publisher, by CCC on publisher's behalf).

13. Objection to Contrary Terms: Publisher hereby objects to any terms contained in any purchase order, acknowledgment, check endorsement or other writing prepared by you, which terms are inconsistent with these terms and conditions or CCC's Billing and Payment terms and conditions. These terms and conditions, together with CCC's Billing and Payment terms and conditions (which are incorporated herein), comprise the entire agreement between you and publisher (and CCC) concerning this licensing transaction. In the event of any conflict between your obligations established by these terms and conditions and those established by CCC's Billing and Payment terms and conditions, these terms and conditions shall control.

14. Revocation: Elsevier or Copyright Clearance Center may deny the permissions described in this License at their sole discretion, for any reason or no reason, with a full refund payable to you. Notice of such denial will be made using the contact information provided by you. Failure to receive such notice will not alter or invalidate the denial. In no event will Elsevier or Copyright Clearance Center be responsible or liable for any costs, expenses or damage incurred by you as a result of a denial of your permission request, other than a refund of the amount(s) paid by you to Elsevier and/or Copyright Clearance Center for denied permissions.

\section{LIMITED LICENSE}

The following terms and conditions apply only to specific license types:

15. Translation: This permission is granted for non-exclusive world English rights only unless your license was granted for translation rights. If you licensed translation rights you may only translate this content into the languages you requested. A professional translator must perform all translations and reproduce the content word for word preserving the integrity of the article. If this license is to re-use 1 or 2 figures then permission is granted for non-exclusive world rights in all languages.

16. Website: The following terms and conditions apply to electronic reserve and author websites: 
Electronic reserve: If licensed material is to be posted to website, the web site is to be password-protected and made available only to bona fide students registered on a relevant course

if: This license was made in connection with a course, This permission is granted for 1 year only. You may obtain a license for future website posting,

All content posted to the web site must maintain the copyright information line on the bottom of each image, A hyper-text must be included to the Homepage of the journal from which you are licensing athttp://www.sciencedirect.com/science/journal/xxxxx or the Elsevier homepage for books athttp://www.elsevier.com, and Central Storage: This license does not include permission for a scanned version of the material to be stored in a central repository such as that provided by Heron/XanEdu.

17. Author website for journals with the following additional clauses:

All content posted to the web site must maintain the copyright information line on the bottom of each image, and the permission granted is limited to the personal version of your paper. You are not allowed to download and post the published electronic version of your article (whether PDF or HTML, proof or final version), nor may you scan the printed edition to create an electronic version. A hyper-text must be included to the Homepage of the journal from which you are licensing athttp://www.sciencedirect.com/science/journal/xxxxx . As part of our normal production process, you will receive an e-mail notice when your article appears on Elsevier's online service ScienceDirect (www.sciencedirect.com). That e-mail will include the article's Digital Object Identifier (DOI). This number provides the electronic link to the published article and should be included in the posting of your personal version. We ask that you wait until you receive this e-mail and have the DOI to do any posting.

Central Storage: This license does not include permission for a scanned version of the material to be stored in a central repository such as that provided by Heron/XanEdu.

18. Author website for books with the following additional clauses: Authors are permitted to place a brief summary of their work online only. A hyper-text must be included to the Elsevier homepage at http://www.elsevier.com . All content posted to the web site must maintain the copyright information line on the bottom of each image. You are not allowed to download and post the published electronic version of your chapter, nor may you scan the printed edition to create an electronic version.

Central Storage: This license does not include permission for a scanned version of the material to be stored in a central repository such as that provided by Heron/XanEdu. 
19. Website (regular and for author): A hyper-text must be included to the Homepage of the journal from which you are licensing at http://www.sciencedirect.com/science/journal/xxxxx. or for books to the Elsevier homepage at http://www.elsevier.com

20. Thesis/Dissertation: If your license is for use in a thesis/dissertation your thesis may be submitted to your institution in either print or electronic form. Should your thesis be published commercially, please reapply for permission. These requirements include permission for the Library and Archives of Canada to supply single copies, on demand, of the complete thesis and include permission for UMI to supply single copies, on demand, of the complete thesis. Should your thesis be published commercially, please reapply for permission.

\section{Other Conditions:}

\section{v1.6}

If you would like to pay for this license now, please remit this license along with your payment made payable to "COPYRIGHT CLEARANCE CENTER" otherwise you will be invoiced within $\mathbf{4 8}$ hours of the license date. Payment should be in the form of a check or money order referencing your account number and this invoice number RLNK501059547.

Once you receive your invoice for this order, you may pay your invoice by credit card. Please follow instructions provided at that time.

Make Payment To:

Copyright Clearance Center

Dept 001

P.O. Box 843006

Boston, MA 02284-3006

For suggestions or comments regarding this order, contact RightsLink Customer Support:customercare@copyright.com or +1-877-622-5543 (toll free in the US) or +1-978-646-2777.

Gratis licenses (referencing $\$ 0$ in the Total field) are free. Please retain this printable license for your reference. No payment is required. 


\section{Permissions to Use Figure 2.4}

\section{ELSEVIER LICENSE TERMS AND CONDITIONS}

Jul 05, 2013

This is a License Agreement between Xiaobo Song ("You") and Elsevier ("Elsevier") provided by Copyright Clearance Center ("CCC"). The license consists of your order details, the terms and conditions provided by Elsevier, and the payment terms and conditions.

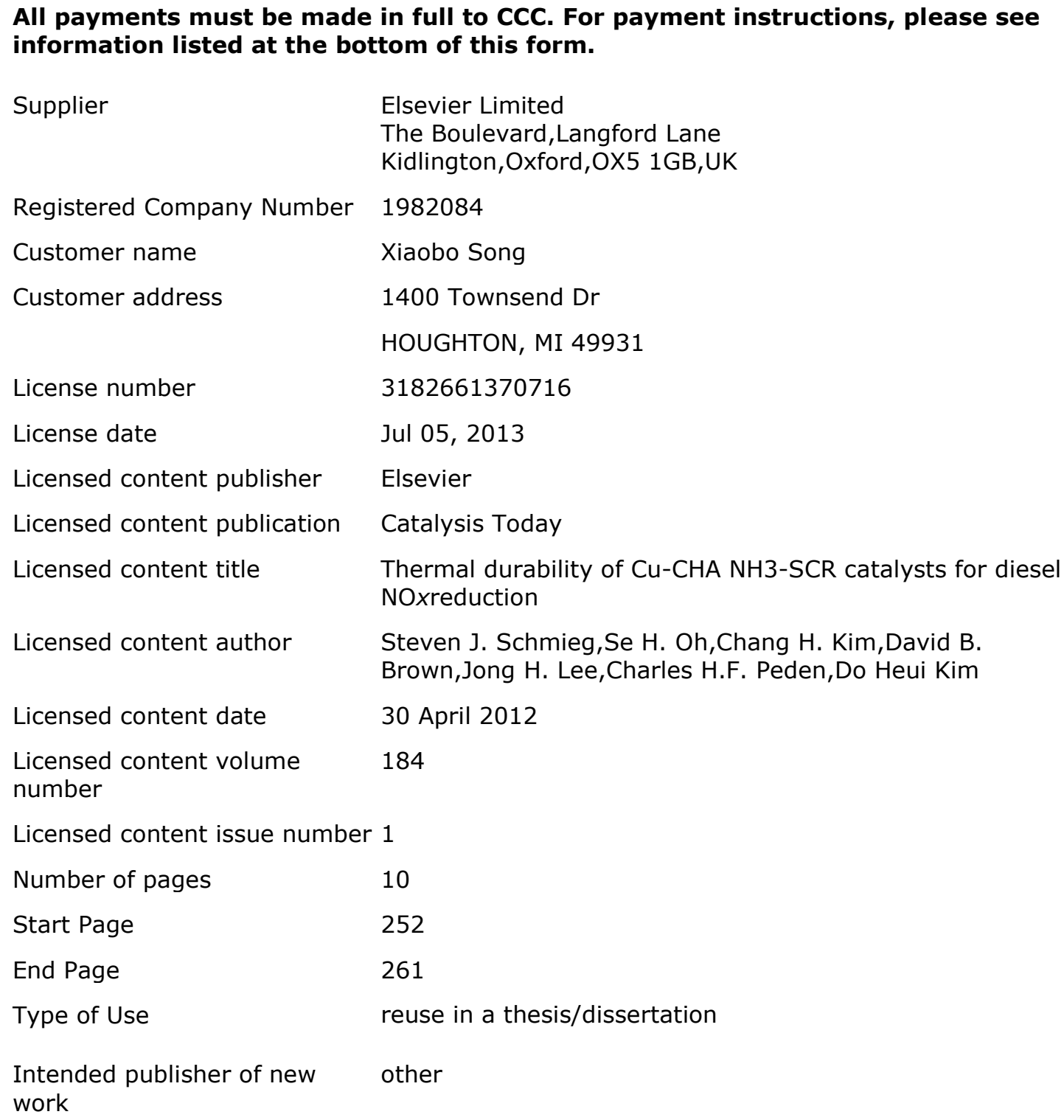




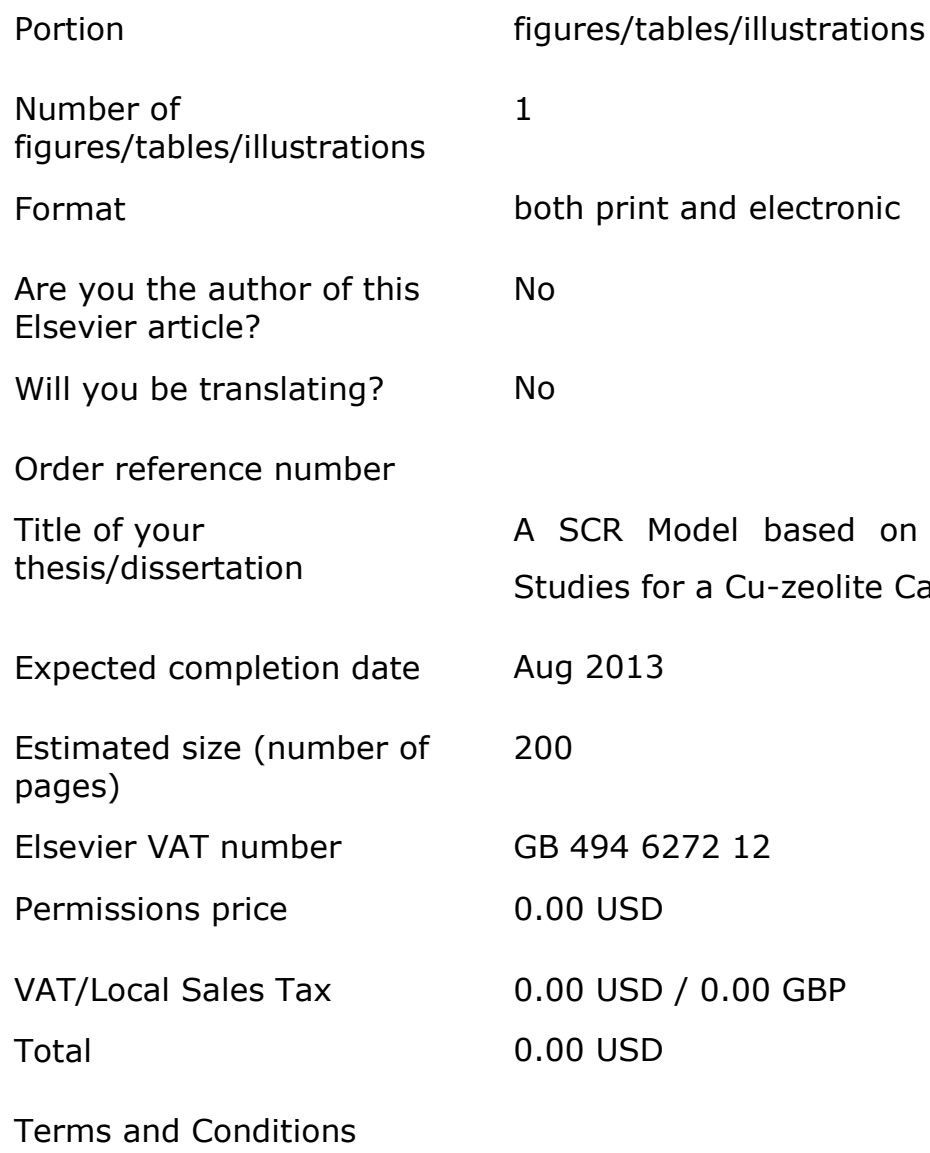

Terms and Conditions

\section{INTRODUCTION}

1. The publisher for this copyrighted material is Elsevier. By clicking "accept" in connection with completing this licensing transaction, you agree that the following terms and conditions apply to this transaction (along with the Billing and Payment terms and conditions established by Copyright Clearance Center, Inc. ("CCC"), at the time that you opened your Rightslink account and that are available at any time at http://myaccount.copyright.com).

\section{GENERAL TERMS}

2. Elsevier hereby grants you permission to reproduce the aforementioned material subject to the terms and conditions indicated.

3. Acknowledgement: If any part of the material to be used (for example, figures) has appeared in our publication with credit or acknowledgement to another source, permission must also be sought from that source. If such permission is not obtained then that material may not be included in your publication/copies. Suitable acknowledgement to the source must be made, either as a footnote or in a reference list at the end of your 
publication, as follows:

"Reprinted from Publication title, Vol /edition number, Author(s), Title of article / title of chapter, Pages No., Copyright (Year), with permission from Elsevier [OR APPLICABLE SOCIETY COPYRIGHT OWNER]." Also Lancet special credit "Reprinted from The Lancet, Vol. number, Author(s), Title of article, Pages No., Copyright (Year), with permission from Elsevier."

4. Reproduction of this material is confined to the purpose and/or media for which permission is hereby given.

5. Altering/Modifying Material: Not Permitted. However figures and illustrations may be altered/adapted minimally to serve your work. Any other abbreviations, additions, deletions and/or any other alterations shall be made only with prior written authorization of Elsevier Ltd. (Please contact Elsevier at permissions@elsevier.com)

6. If the permission fee for the requested use of our material is waived in this instance, please be advised that your future requests for Elsevier materials may attract a fee.

7. Reservation of Rights: Publisher reserves all rights not specifically granted in the combination of (i) the license details provided by you and accepted in the course of this licensing transaction, (ii) these terms and conditions and (iii) CCC's Billing and Payment terms and conditions.

8. License Contingent Upon Payment: While you may exercise the rights licensed immediately upon issuance of the license at the end of the licensing process for the transaction, provided that you have disclosed complete and accurate details of your proposed use, no license is finally effective unless and until full payment is received from you (either by publisher or by CCC) as provided in CCC's Billing and Payment terms and conditions. If full payment is not received on a timely basis, then any license preliminarily granted shall be deemed automatically revoked and shall be void as if never granted. Further, in the event that you breach any of these terms and conditions or any of CCC's Billing and Payment terms and conditions, the license is automatically revoked and shall be void as if never granted. Use of materials as described in a revoked license, as well as any use of the materials beyond the scope of an unrevoked license, may constitute copyright infringement and publisher reserves the right to take any and all action to protect its copyright in the materials.

9. Warranties: Publisher makes no representations or warranties with respect to the licensed material.

10. Indemnity: You hereby indemnify and agree to hold harmless publisher and CCC, and their respective officers, directors, employees and agents, from and against any and 
all claims arising out of your use of the licensed material other than as specifically authorized pursuant to this license.

11. No Transfer of License: This license is personal to you and may not be sublicensed, assigned, or transferred by you to any other person without publisher's written permission.

12. No Amendment Except in Writing: This license may not be amended except in a writing signed by both parties (or, in the case of publisher, by CCC on publisher's behalf).

13. Objection to Contrary Terms: Publisher hereby objects to any terms contained in any purchase order, acknowledgment, check endorsement or other writing prepared by you, which terms are inconsistent with these terms and conditions or CCC's Billing and Payment terms and conditions. These terms and conditions, together with CCC's Billing and Payment terms and conditions (which are incorporated herein), comprise the entire agreement between you and publisher (and CCC) concerning this licensing transaction. In the event of any conflict between your obligations established by these terms and conditions and those established by CCC's Billing and Payment terms and conditions, these terms and conditions shall control.

14. Revocation: Elsevier or Copyright Clearance Center may deny the permissions described in this License at their sole discretion, for any reason or no reason, with a full refund payable to you. Notice of such denial will be made using the contact information provided by you. Failure to receive such notice will not alter or invalidate the denial. In no event will Elsevier or Copyright Clearance Center be responsible or liable for any costs, expenses or damage incurred by you as a result of a denial of your permission request, other than a refund of the amount(s) paid by you to Elsevier and/or Copyright Clearance Center for denied permissions.

\section{LIMITED LICENSE}

The following terms and conditions apply only to specific license types:

15. Translation: This permission is granted for non-exclusive world English rights only unless your license was granted for translation rights. If you licensed translation rights you may only translate this content into the languages you requested. A professional translator must perform all translations and reproduce the content word for word preserving the integrity of the article. If this license is to re-use 1 or 2 figures then permission is granted for non-exclusive world rights in all languages.

16. Website: The following terms and conditions apply to electronic reserve and author websites: 
Electronic reserve: If licensed material is to be posted to website, the web site is to be password-protected and made available only to bona fide students registered on a relevant course

if: This license was made in connection with a course, This permission is granted for 1 year only. You may obtain a license for future website posting,

All content posted to the web site must maintain the copyright information line on the bottom of each image, A hyper-text must be included to the Homepage of the journal from which you are licensing athttp://www.sciencedirect.com/science/journal/xxxxx or the Elsevier homepage for books athttp://www.elsevier.com, and Central Storage: This license does not include permission for a scanned version of the material to be stored in a central repository such as that provided by Heron/XanEdu.

17. Author website for journals with the following additional clauses:

All content posted to the web site must maintain the copyright information line on the bottom of each image, and the permission granted is limited to the personal version of your paper. You are not allowed to download and post the published electronic version of your article (whether PDF or HTML, proof or final version), nor may you scan the printed edition to create an electronic version. A hyper-text must be included to the Homepage of the journal from which you are licensing athttp://www.sciencedirect.com/science/journal/xxxxx . As part of our normal production process, you will receive an e-mail notice when your article appears on Elsevier's online service ScienceDirect (www.sciencedirect.com). That e-mail will include the article's Digital Object Identifier (DOI). This number provides the electronic link to the published article and should be included in the posting of your personal version. We ask that you wait until you receive this e-mail and have the DOI to do any posting.

Central Storage: This license does not include permission for a scanned version of the material to be stored in a central repository such as that provided by Heron/XanEdu.

18. Author website for books with the following additional clauses: Authors are permitted to place a brief summary of their work online only. A hyper-text must be included to the Elsevier homepage at http://www.elsevier.com . All content posted to the web site must maintain the copyright information line on the bottom of each image. You are not allowed to download and post the published electronic version of your chapter, nor may you scan the printed edition to create an electronic version.

Central Storage: This license does not include permission for a scanned version of the material to be stored in a central repository such as that provided by Heron/XanEdu. 
19. Website (regular and for author): A hyper-text must be included to the Homepage of the journal from which you are licensing at http://www.sciencedirect.com/science/journal/xxxxx. or for books to the Elsevier homepage at http://www.elsevier.com

20. Thesis/Dissertation: If your license is for use in a thesis/dissertation your thesis may be submitted to your institution in either print or electronic form. Should your thesis be published commercially, please reapply for permission. These requirements include permission for the Library and Archives of Canada to supply single copies, on demand, of the complete thesis and include permission for UMI to supply single copies, on demand, of the complete thesis. Should your thesis be published commercially, please reapply for permission.

\section{Other Conditions:}

\section{v1.6}

If you would like to pay for this license now, please remit this license along with your payment made payable to "COPYRIGHT CLEARANCE CENTER" otherwise you will be invoiced within $\mathbf{4 8}$ hours of the license date. Payment should be in the form of a check or money order referencing your account number and this invoice number RLNK501059545.

Once you receive your invoice for this order, you may pay your invoice by credit card. Please follow instructions provided at that time.

Make Payment To:

Copyright Clearance Center

Dept 001

P.O. Box 843006

Boston, MA 02284-3006

For suggestions or comments regarding this order, contact RightsLink Customer Support:customercare@copyright.com or +1-877-622-5543 (toll free in the US) or +1-978-646-2777.

Gratis licenses (referencing $\$ 0$ in the Total field) are free. Please retain this printable license for your reference. No payment is required. 